\title{
Ethynyl BenziodoXolones (EBX) as Reagents for the Ethynylation of Stabilized Enolates
}

\author{
Davinia Fernández González, ${ }^{\mathrm{a}}$ Jonathan P. Brand, ${ }^{\mathrm{a}}$ Régis Mondière, ${ }^{\mathrm{b}}$ and Jérôme \\ Waser $^{\text {a }}$ Fer
}

a Laboratory of Catalysis and Organic Synthesis, Ecole Polytechnique Fédérale de Lausanne, CH-1015,

Switzerland. Fax: +41 (0)21 69397 00; Phone: +41 (0)21 69393 88; E-mail: jerome.waser@epfl.ch

b Syngenta Crop Protection Münchwilen AG, Research Chemistry, Schaffhauserstrasse, CH-4332 Stein, Switzerland

Received:

Supporting information for this article is available on the WWW under http://dx.doi.org/10.1002/adsc.201

\begin{abstract}
Herein, we report a detailed study on the electrophilic alkynylation of cyclic keto-esters and amides with ethynylbenziodoxolone (EBX) reagents. The structure and stability of this class of reagents is first described more in details. DSC experiments showed a strong exothermic decomposition with EBX reagents, leading to guidelines for the safe use of these compounds. The extension of the method to aromatic alkynes and a broad range of benziodoxol(on)e reagents is then reported.

Based on our preliminary results using cinchona-based phase-transfer catalysts, the enantioselective alkynylation of cyclic keto-esters could be achieved. Binaphthyl-derived ammonium catalysts developed by Maruoka and co-workers gave the highest asymmetric induction with up to $79 \%$ ee for an indanone-derived keto-ester.
\end{abstract}

\section{Introduction}

In the last decades, acetylene chemistry has been developed considerably. The unique electronic properties and the rigid linear structure of alkynes have led to important applications not only in the field of organic chemistry, but also in biochemistry and material sciences. ${ }^{[1]}$ Furthermore, there are numerous methods for triple bond functionalization, providing a versatile access to more complex molecules and making acetylenes very interesting intermediates in organic synthesis. ${ }^{[2]}$

As terminal acetylenes are easily deprotonated, the addition of an acetylide anion to an electrophile is one of the most often used reactions for their synthesis. For example, the addition of acetylides to carbonyls gives an efficient access to propargylic alcohols and catalytic asymmetric versions of this reaction have also been developed (Scheme $1, \mathbf{A}){ }^{[3]}$ When considering the large variety of nucleophiles available in organic chemistry, an electrophilic alkynylation method would be highly desirable (Scheme 1, B). However, addition of a nucleophile on an alkyne electrophile has been only rarely used. In this case, the Umpolung of the reactivity of
Throughout this work, asymmetric induction was observed only in the case of benziodoxolone reagents, demonstrating their superiority over conventional alkynyliodonium salts. The deeper understanding gained about the factors leading to higher asymmetric induction will be very useful in the future to develop a truly general and highly enantioselective alkynylation method.

Keywords: acetylene; hypervalent iodine; asymmetric reaction; quaternary centers; Umpolung acetylenes is required. ${ }^{[4]}$ A particularly interesting class of nucleophiles is constituted by $\alpha$-disubstituted carbonyl compounds, as the addition of the triple bond in alpha position allows the formation of allcarbon quaternary centers, which cannot be accessed using the normal polarity of acetylenes.

A) Classical Reactivity of Acetylenes<smiles>[R]C#CC([R])O</smiles>

B) Non-Conventional Reactivity (Umpolung)<smiles>[R]C#CC([R])([R])C([R])=O</smiles>

Scheme 1. Classical and non-conventional reactivity of acetylenes with carbonyl compounds.

The rare methods reported for the Umpolung of acetylenes for the alkynylation of enolates are based on the use of halogen acetylenes, ${ }^{[5]}$ lead acetylide reagents ${ }^{[6]}$ and alkynyliodonium salts ${ }^{[7]}$ as electrophilic reagents to introduce the triple bond. 
However, the scope reported for the $\alpha$-alkynylation of carbonyl compounds using these methods is limited. Furthermore, there is only one single report of an enantioselective method for the alkynylation of keto esters to generate all-carbon chiral quaternary centers. It was achieved by Jørgensen and co-workers in 2007 using a chiral phase-transfer catalyst. ${ }^{[8]}$ However, alkynylation was possible only using acetylenes bearing an electron-withdrawing group, such as propionic acid derivatives. The synthetically most versatile free acetylenes could not be accessed directly.

Consequently, searching for new electrophilic acetylene synthons for a more general and efficient alkynylation in alpha position to carbonyl functional groups is essential to make the Umpolung alkynylation approach more synthetically useful. Recently, we have demonstrated the exceptional acetylene-transfer ability of 1[(trimethylsilyl)ethynyl]-1,2-benziodoxol-3(1H)-one (TMS-EBX, 1a, Scheme 2) with soft enolates. ${ }^{[9]}$ This method gave direct access to the synthetically most versatile free acetylenes under mild reaction conditions using tetrabutylammonium fluoride (TBAF) both as an activating agent and a base. The use of a cyclic benziodoxolone reagent was essential to improve both efficiency and scope of previously reported methods based on alkynyl iodonium salts. ${ }^{[7]}$ Works from our group and others have further demonstrated the utility of EBX reagents in a broad range of electrophilic alkyne transfer reactions. ${ }^{[10]}$

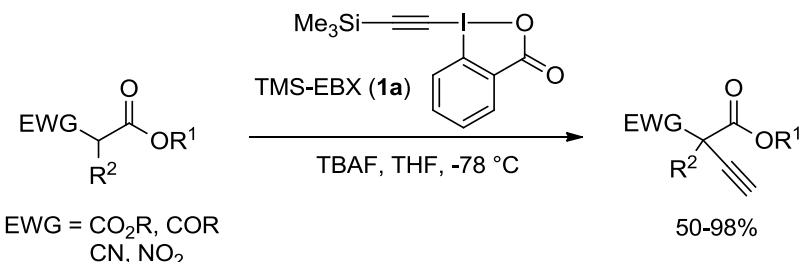

Scheme 2. Alkynylation of activated carbonyl compounds with TMS-EBX (1a).

Alkynylation with TMS-EBX (1a) was also successful under phase-transfer conditions using a chiral cinchona ammonium salt as catalyst. Although good conversion was observed under these conditions, only a weak asymmetric induction $(40 \%$ ee $)$ was obtained. ${ }^{[9]}$ Very recently, Veselý and co-workers applied this method for the alkynylation of $\alpha$ fluorinated sulfone derivatives with up to $61 \%$ ee. ${ }^{[11]}$ To better understand these results and fully explore the potential of this new class of reagents, we decided to investigate more in detail the reactivity of different new EBX reagents and the influence of substrate structure on reactivity and asymmetric induction.

Herein, we report the results of these studies, which demonstrated that the alkynylation is successful for a broad range of benziodoxolone reagents and can be extended to the transfer of aromatic acetylenes. We also give a detailed study of the thermal stability of these reagents, which defines more precisely their safety profile. In the case of cyclic keto-ester, a more in-depth study of the influence of the substrate structure and diverse phase-transfer catalysts led to an increase of the asymmetric induction up to $79 \%$ ee using a binaphthyl-derived ammonium catalyst.

\section{Results and Discussion}

\section{Reagent structure and stability}

We initiated our study by evaluating the reactivity of different silylated hypervalent iodine reagents using our previously developed conditions using TBAF both as base and fluoride source (Scheme 3 ) ${ }^{99}$ In this case, the reaction of $\beta$-keto ester $\mathbf{2 a}$ with hypervalent iodine reagents $\mathbf{1 a}, \mathbf{1 b}$ and $\mathbf{4}$ gave only free acetylene 3a in good yield. The reaction with cyclic iodane compounds, especially TMS-EBX (1a), is faster than when using iodonium salts. This result is somewhat surprising, as alkynyliodonium could be reasonably expected to be more reactive, due to their higher ionic character.
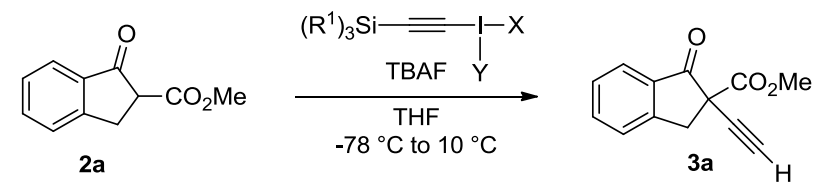

2a
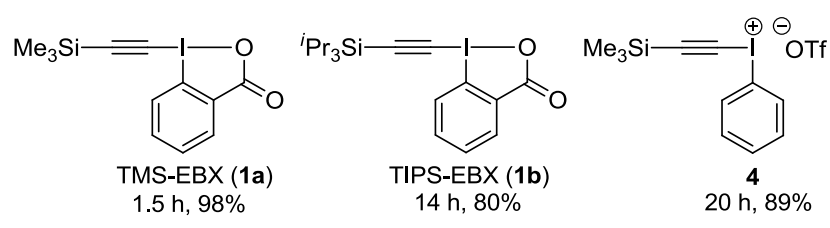

Scheme 3. Alkynylation of keto-ester 2a using EBX reagents $\mathbf{1 a}$ and $\mathbf{1 b}$ and alkynyliodonium salt $\mathbf{4}$.

In order to better understand the exceptional reactivity of TMS-EBX (1a), we first examined more in detail its solid state structure. TMS-EBX (1a) could be recrystallized from a mixture of hexane $/ \mathrm{CH}_{2} \mathrm{Cl}_{2}$ (1:1) by slow evaporation of the solvent mixture to give high quality crystals for X-ray analysis (Figure 1). ${ }^{[12]} \mathrm{X}$-ray diffraction confirmed that 1a had a cyclic structure with a three-coordinated T-shaped hypervalent iodine atom. The distorted trigonal bipyramide geometry $(\mathrm{C}(8)-\mathrm{I}(1)-\mathrm{O}(1)$ angle: $18^{\circ}$, torsion angle: $3^{\circ}$ ) let the triple bond free for attack by the nucleophile in the reaction. The bond lengths around the iodine atom are 2.128(2) $\AA$ for $\mathrm{I}(1)-\mathrm{C}(1), 2.062(2) \AA$ for I(1)-C(8) and 2.355(1) $\AA$ for $\mathrm{I}(1)-\mathrm{O}(1)$. The length of the $\mathrm{I}(1)-\mathrm{O}(1)$ bond indicates a strong interaction between $\mathrm{I}(1)$ and $\mathrm{O}(1)$ and is significantly shorter than the reported bond length for alkynyliodonium salts $(2.620 \AA) \cdot{ }^{[13]}$ However, it is still longer than a truly covalent I-O bond. Furthermore, the two C(7)-O bonds are of different lengths, but the difference is small (1.290(2) for C(7)$\mathrm{O}(1)$ and $1.242(2)$ for $\mathrm{C}(7)-\mathrm{O}(2)$. The stronger partial 
bond character of the $\mathrm{I}(1)-\mathrm{O}(1)$ bond in comparison to alkynyliodonium salts is probably one of the important sources of the exceptional reactivity of TMS-EBX (1a). Non-cyclic alkynyliodonium salts with similar carboxylate substituents are not stable, as they react immediately to form alkynyl esters. ${ }^{[14]}$

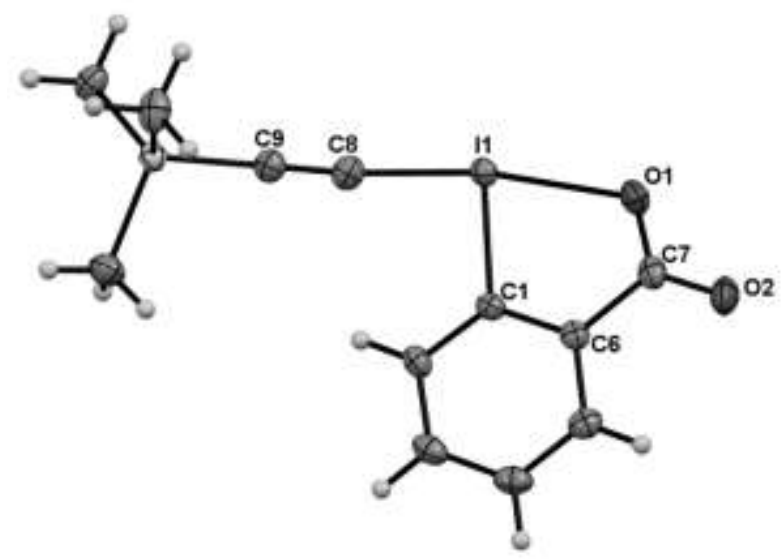

Figure 1. X-ray structure of TMS-EBX (1a) showing one molecule. Selected bond lengths: I(1)-C(1): 2.128(2), I(1)$\mathrm{C}(8): 2.062$ (2), I(1)-O(1): 2.355(1), C(8)-C(9): 1.218(2), $\mathrm{C}(7)-\mathrm{O}(1): 1.290(2), \mathrm{C}(7)-\mathrm{O}(2): 1.242(2)$.

In the solid state, TMS-EBX (1a) is further stabilized by strong intermolecular interactions. In particular, the distance between the iodine atom and the carbonyl group of the next molecule is only 2.902 $\AA$, indicating a relatively strong interaction. This results in a pseudo-polymeric structure of TMS-EBX (1a) and further explains its high crystallinity. However, this information obtained in the solid state does not allow any definitive conclusions about the behaviour of the reagent in solution.

All hypervalent iodine reagents are high energy compounds. It is consequently important to have enough data on their stability to allow their safe handling. The thermal stability of TMS- and TIPSEBX reagents $\mathbf{1 a}$ and $\mathbf{1 b}$ was consequently further investigated by DSC studies.

For what concerns TMS-EBX (1a), an exothermic behaviour was observed from $117{ }^{\circ} \mathrm{C}$ to $181{ }^{\circ} \mathrm{C}(596$ $\mathrm{J} / \mathrm{g}) .{ }^{[15]}$ Consequently, great care has to be taken when using TMS-EBX (1a), as these data very likely indicate a risk of runaway scenario. The DSC device that we used has a sensitivity of $10 \mathrm{~W} / \mathrm{kg}$ meaning that as a rule of thumb, with such a sensitivity a safety margin (decrease of onset temperature) of $60{ }^{\circ} \mathrm{C}$ is generally considered sufficient for production scale. In our case this would mean to run the reaction below $57{ }^{\circ} \mathrm{C}$ to minimize major safety issue (this should be checked by Process risk analyses). However this does not take into account the fact that this degradation may have a lower onset if catalyzed by metals or impurities.
In the case of TIPS-EBX (1b) a large exothermic behaviour was observed by DSC from $135^{\circ} \mathrm{C}$ to 245 -C $(532 \mathrm{~J} / \mathrm{g})$. Consequently, using the same safety margin as before, reactions run with the TIPS-EBX (1b) below $75^{\circ} \mathrm{C}$ could be regarded as safe from the thermal point of view provided no metal catalyzed or impurity catalyzed decomposition is occurring. Finally, several qualitative shock sensitivity tests on both TMS- and TIPS-EBX reagents $\mathbf{1 a}$ and $\mathbf{1 b}$ did not lead to any explosion using the compounds synthesized in our laboratory.

We can conclude from these studies that the manipulation of reagents $\mathbf{1 a}$ and $\mathbf{1 b}$ is relatively "thermally safe" at temperatures below respectively $57{ }^{\circ} \mathrm{C}$ and $75^{\circ} \mathrm{C}$. However, even in this case great care should be taken when manipulating these reagents, especially on larger scale, as impurities and metals could potentially act as catalyst and initiate the onset of the decomposition at lower temperatures.

\section{Alkynylation reactions with modified Ethynyl BenziodoXolones (EBX)}

Having examined the structure and stability of the parent EBX reagents, we then decided to examine the influence of chemical modifications both on the benzene core and the benziodoxolone heterocycle in the alkynylation process. Due to their enhanced stability, we decided to focus on triisopropylsilylsubstituted benziodoxolone and benziodoxole reagents for these studies. These reagents had been synthesized previously in our group. ${ }^{[10 \mathrm{e}]}$

We started our investigation with the alkynylation of tert-butyl $\beta$-keto ester $\mathbf{2 b}$ under our standard conditions with TBAF as reagent (Table 1). We observed that both reagent 1c bearing an electron withdrawing and reagent $\mathbf{1 d}$ bearing an electrondonating group on the benzene ring afforded alkynylated product $\mathbf{3 b}$ in good yields (entries 2-3). The reaction was faster with electron-deficient reagent 1c, however. Methylated TIPS-EBX reagents 1e and 1f led to the formation of the ethynylated product $\mathbf{3 b}$ in moderate yields and relatively short reaction times (entries 4-5). Finally, we found that TIPS benziodioxole reagents $\mathbf{5}$ and $\mathbf{6}$ are also efficient alkynylation reagents under these conditions, although in this case the alkynylation reaction is slower (entries 6-7). When considering that a stronger base is liberated upon alkynylation, these results could be interesting in the future to extend the scope of the reaction to less acidic substrates. Furthermore, this result is in sharp contrast to gold-catalyzed reactions involving EBX reagents, as in this case benziodoxole-derived reagents were not efficient alkyne-transfer reagents. ${ }^{[10 \mathrm{e}]}$

In a second step, we examined if the scope of the alkynylation reaction could be extended to aromatic acetylenes (Table 2). We were delighted to see that the reaction with phenyl-EBX reagent 8a afforded the corresponding phenyl acetylene product 7a using the TBAF conditions (entry 1 ). We shortly investigated if modification of the benzene ring would lead to better yields in the alkynylation reaction. The new reagents 
8b-d were consequently synthesized using known procedures. ${ }^{[10 e, 16]}$ Reagents $\mathbf{8 b}$ and $\mathbf{8 c}$ with electronwithdrawing groups on the benzene ring of the benziodoxolone gave low yields (entries 2-3). The introduction of electron-donating groups in $\mathbf{8 d}$, on the other hand, led to a small increase in yield (entry 4). The influence of substituents on the benzene ring attached to the acetylene was even stronger. No reaction was observed for reagent 9 with an electronwithdrawing nitro group (entry 5). Unfortunately, the corresponding reagent bearing an electron-donating methoxy group could not be synthesized, as it was not stable. In contrast, we observed that reagent $\mathbf{1 0}$ with a mesitylene aromatic group gave the highest yield observed so far (85\%, entry 6$)$.

Table 1. Alkynylation of keto ester $\mathbf{2 b}$ using EBX reagents with a modified benziodoxole ring.

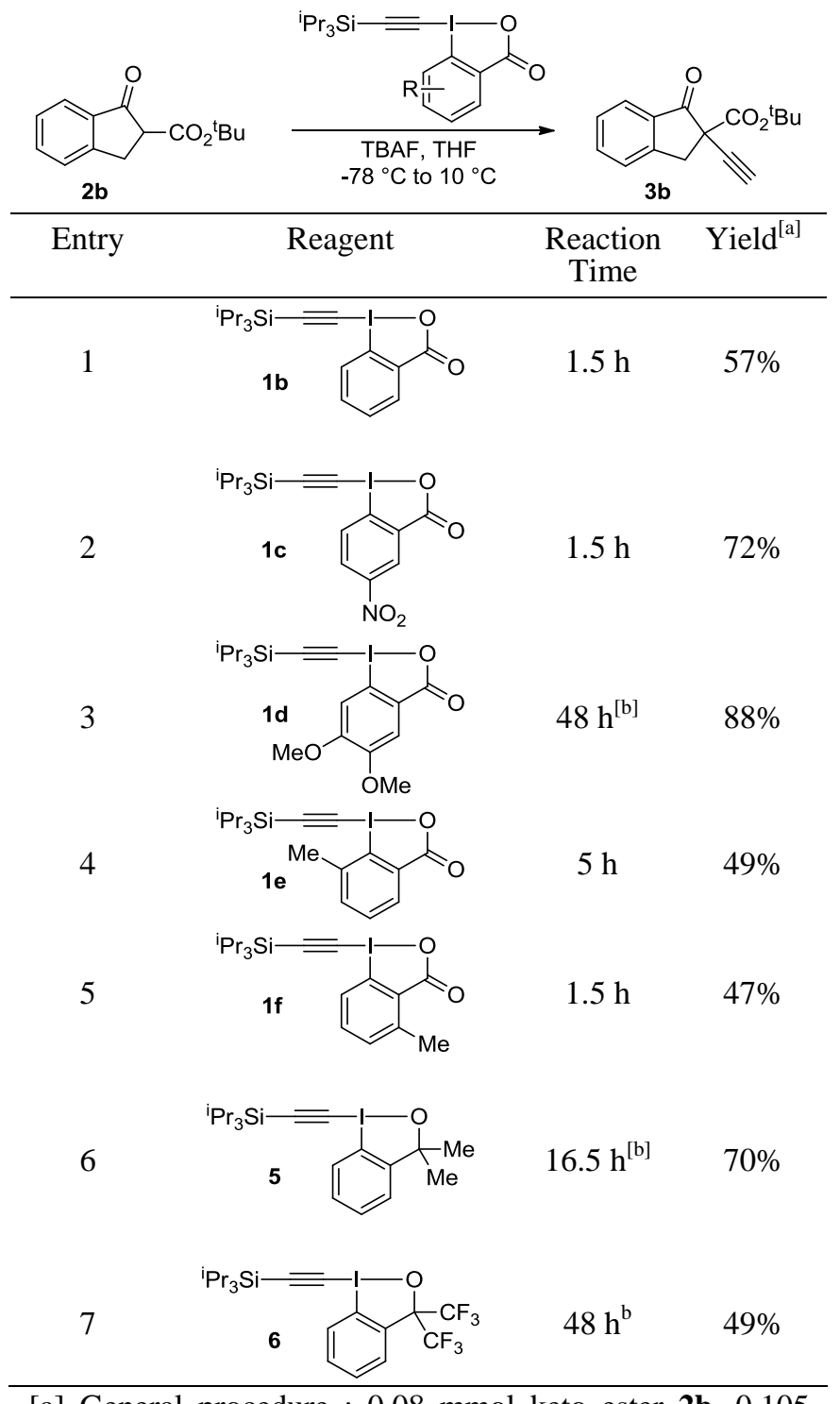

[a] General procedure : $0.08 \mathrm{mmol}$ keto ester $\mathbf{2 b}, 0.105$ mmol iodane reagent, $0.105 \mathrm{mmol}$ TBAF, $1.35 \mathrm{~mL}$ THF at $-78{ }^{\circ} \mathrm{C}$ under nitrogen. [b] Reaction completed at $10{ }^{\circ} \mathrm{C}$.
When considering that TBAF had been used because of its higher silicon affinity, there was no real requirement for such a reagent when using aromatic alkynyl reagents. We consequently examined the use of $\mathrm{Bu}_{4} \mathrm{NOH}$ to promote the alkynylation reaction using $\mathbf{8 a}$ and keto ester $\mathbf{2 b}$ under similar conditions (Scheme 4). We observed that, even if the reaction afforded the product $7 \mathbf{a}$, the obtained yield was lower than for the same reaction with TBAF. This could be due to the fact that hydrated TBAF is a milder base, which prevented a competitive decomposition of the reagent.

Table 2. Alkynylation of keto ester $\mathbf{2 b}$ using modified EBX reagents derived from aromatic alkynes.

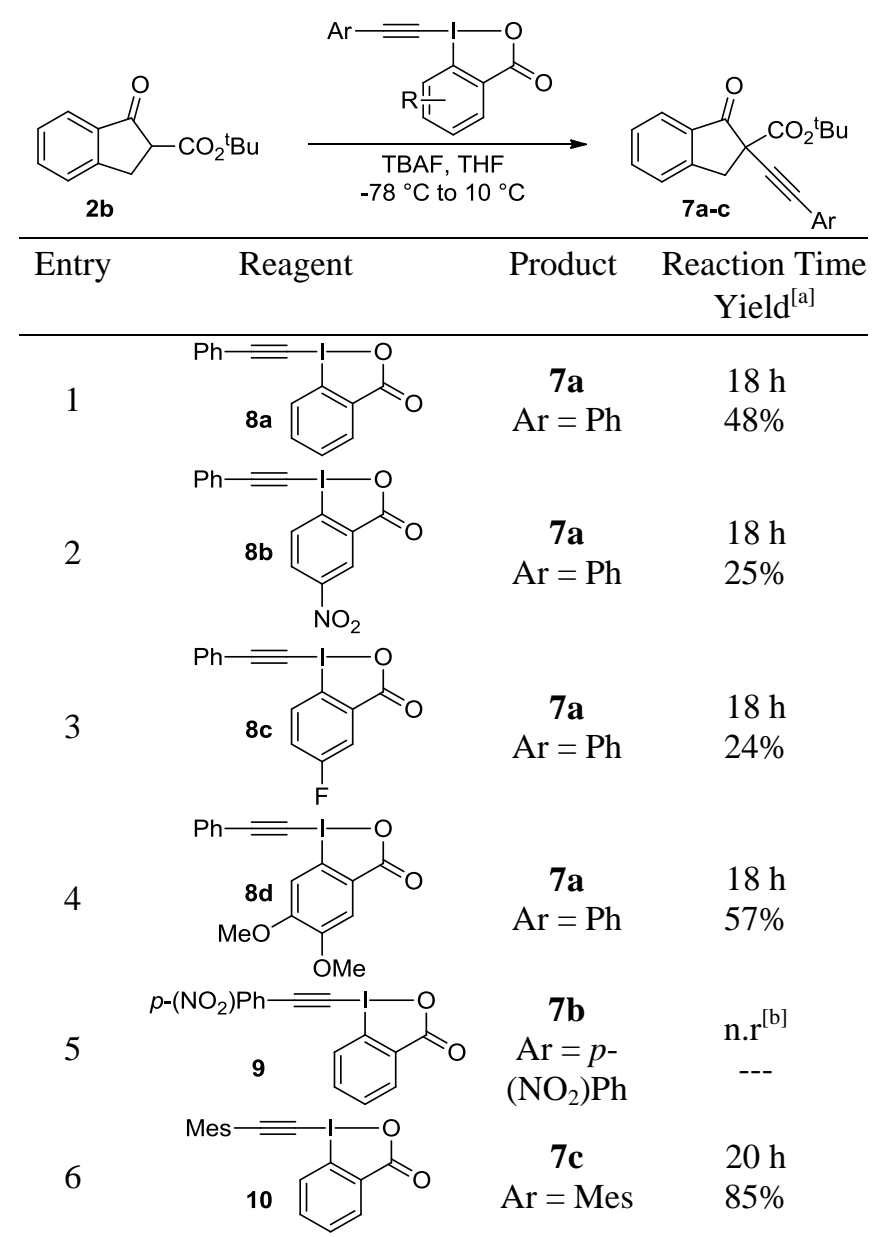

[a] General procedure: $0.08 \mathrm{mmol}$ keto ester $2 \mathbf{b}, 0.105$ mmol iodane reagent, $0.105 \mathrm{mmol}$ TBAF, $1.35 \mathrm{~mL}$ THF at $-78^{\circ} \mathrm{C}$ to $10{ }^{\circ} \mathrm{C}$ under nitrogen. [b] No reaction.
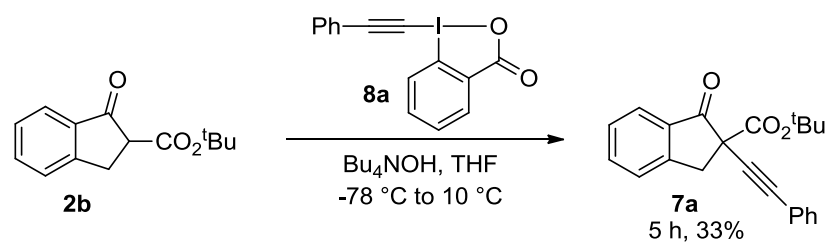

Scheme 4. Alkynylation of keto ester $\mathbf{2 b}$ using $\mathrm{Bu}_{4} \mathrm{NOH}$ as base. 


\section{Asymmetric Akynylation: Catalyst optimization and influence of substrate structure.}

In our previous work, we had achieved the alkynylation of substrate $\mathbf{2 b}$ with up to $40 \%$ ee using a cinchona-based phase-transfer catalyst and TMSEBX (1a). Despite extensive efforts, we were not able to improve this result using other cinchonaderived catalysts. We consequently decided to examine other classes of potential phase-transfer catalysts. In particular, we became interested by the chiral tetraaminophosphonium salts introduced by Ooi and co-workers in $2008^{[17]}$ and the binaphthylbased ammoniums developed by Maruoka and coworkers. ${ }^{[18]}$ Indeed, the first results obtained using these catalysts were promising (Scheme 5). Phosphonium catalysts 11a and $\mathbf{1 1 b}$ led to an increase of the enantioselectivity up to $51 \%$. The most efficient catalyst was commercially available binaphthyl ammonium 12, for which $\mathbf{3 b}$ was obtained with $59 \%$ enantioselectivity.

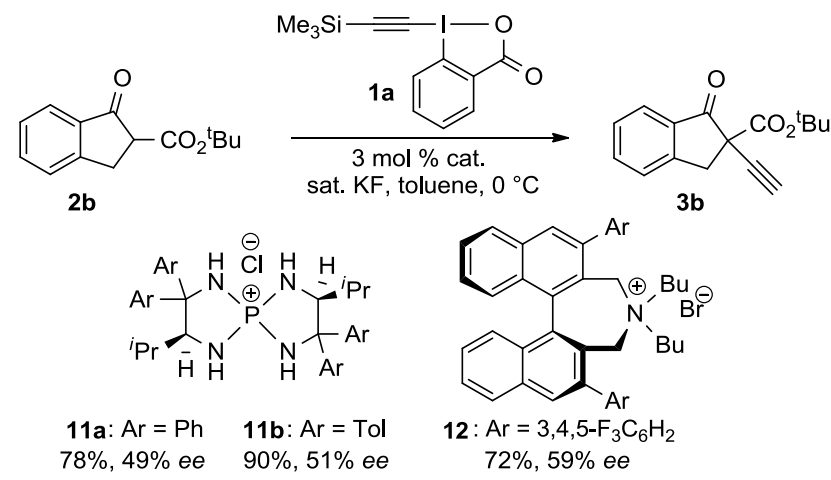

Scheme 5. Asymmetric alkynylation of keto ester $\mathbf{2 b}$.

With this promising lead result obtained with Maruoka's catalyst 12, we then examined the effect of reaction conditions on the enantioselectivity (Table 3). The influence of solvents was investigated first. A slight decrease in the yield and enantioselectivity was observed in dichloromethane (entry 2). In contrast, the enantioselective excess could be increased from $59 \%$ to $65 \%$ ee using xylene as solvent (entry 3 ). No further increase was observed when using mesitylene (entry 4).

The catalyst loading had no strong influence on the reaction outcome: using $1.5 \mathrm{~mol} \%$ of catalyst 12 instead of $3 \mathrm{~mol} \%$, the yield decreased slightly to $60 \%$ and the enantiomeric excess was nearly identical (62\% instead of $65 \%$, entry 5 ).

The influence of the base was investigated next. If a direct background reaction would occur, a reduction of the base concentration could lead to higher enantiomeric excess. However, a lower yield was observed when using a $1 \mathrm{M}$ solution of $\mathrm{KF}$, and no increase in the enantiomeric excess was obtained (entry 6). Changing the base to $\mathrm{K}_{2} \mathrm{CO}_{3}$ led to a mixture of silylated and free acetylenes with similar ee (entry 7). The use of solid KF as base at low temperature surprisingly led to a high yield of product 3b, albeit with lower ee (entry 8). The ee could be restored by using alcohols as additives (entries 9 and 10). Although the results using alcohol additives and solid bases at low temperature still warrant further investigation, we decided to continue our optimization with the more convenient liquidliquid phase transfer conditions with saturated KF solutions.

Table 3. Influence of reaction conditions on the asymmetric alkynylation of $\mathbf{2 b}$ with catalyst $\mathbf{1 2}$.

\begin{tabular}{|c|c|c|c|c|}
\hline Enty & Base & $\begin{array}{c}\text { Solvent } \\
\text { Temperature }\end{array}$ & Yield $^{[\mathrm{a}]}$ & $e e$ \\
\hline 1 & $\mathrm{KF}_{\text {(sat.) }}$ & $\begin{array}{c}\text { toluene } \\
0^{\circ} \mathrm{C}\end{array}$ & $72 \%$ & $59 \%$ \\
\hline 2 & $\mathrm{KF}_{\text {(sat.) }}$ & $\begin{array}{c}\mathrm{CH}_{2} \mathrm{Cl}_{2} \\
0^{\circ} \mathrm{C}\end{array}$ & $60 \%$ & $54 \%$ \\
\hline 3 & $\mathrm{KF}_{\text {(sat.) }}$ & $\begin{array}{c}\text { xylene } \\
0^{\circ} \mathrm{C}\end{array}$ & $70 \%$ & $65 \%$ \\
\hline 4 & $\mathrm{KF}_{\text {(sat.) }}$ & $\begin{array}{c}\text { mesitylene } \\
0^{\circ} \mathrm{C}\end{array}$ & $64 \%$ & $64 \%$ \\
\hline 5 & $\mathrm{KF}_{\text {(sat.) }}$ & $\begin{array}{c}\text { xylene } \\
0^{\circ} \mathrm{C}\end{array}$ & $60 \%^{[\mathrm{b}]}$ & $62 \%$ \\
\hline 6 & $\mathrm{KF} 1 \mathrm{M}$ & $\begin{array}{c}\text { xylene } \\
0^{\circ} \mathrm{C}\end{array}$ & $30 \%$ & $61 \%$ \\
\hline 7 & $\mathrm{~K}_{2} \mathrm{CO}_{3} 1 \mathrm{M}$ & $\begin{array}{c}\text { xylene } \\
0^{\circ} \mathrm{C}\end{array}$ & mixt. ${ }^{[c]}$ & $64 \% \%^{[\mathrm{d}]}$ \\
\hline 8 & $\mathrm{KF}_{\text {(sat.) }}$ & $\begin{array}{l}\text { xylene } \\
-78^{\circ} \mathrm{C}\end{array}$ & $91 \%$ & $48 \%$ \\
\hline 9 & $\mathrm{KF}_{\text {(sat.) }}$ & $\begin{array}{c}\text { xylene } / \mathrm{MeOH} \\
-50^{\circ} \mathrm{C}\end{array}$ & $73 \%$ & $67 \%$ \\
\hline 10 & $\mathrm{KF}_{\text {(sat.) }}$ & $\begin{array}{c}\text { xylene } / \mathrm{BuOH} \\
-50{ }^{\circ} \mathrm{C}\end{array}$ & $82 \%$ & $64 \%$ \\
\hline
\end{tabular}

[a] General procedure: $0.2 \mathrm{mmol} \mathbf{2 b}, 1.3$ equiv. reagent 1a, base solution $(1 \mathrm{~mL}), 3 \mathrm{~mol} \%$ of catalyst 12 , solvent $(50$ $\mathrm{mM}$ in 2b) under nitrogen. [b] With $1.5 \mathrm{~mol} \%$ of 12. [c] Mixture of protected and deprotected alkyne product. [d] $e e$ of deprotected product.

We then shortly investigated if the reagent structure had an influence on the asymmetric induction (Scheme 6). Using TIPS-EBX (1b), the silylated product could be obtained exclusively using potassium carbonate as a base, but not change in enantioselectivity was observed (1). Using potassium fluoride as a base and different benziodoxol(on)e reagents, a mixture of silylated and free acetylenes was usually obtained, which was directly converted to the free acetylenes using TBAF. However, all the investigated reagents led to lower enantioselectivity (2).

During our studies, we had also observed that the bulky tert-butyl ester group on $\mathbf{2 b}$ was required for asymmetric induction. As a next step, we wondered if further fine tuning of this substituent would allow us to reach higher enantioselectivity. We first turned to the introduction of other bulky ester groups on the indanone (Table 4, entries 1-4). We were pleased to see that replacement of one methyl group on the tert- 
butyl by a phenyl group led to an increase of the enantiomeric excess up to $79 \%$ (entry 2). Unfortunately, using an even more bulky adamantyl or an anthracenylmethylene group led to lower asymmetric induction (entries 3 and 4). Finally, we investigated if structurally more rigid amides would lead to a better asymmetric induction. Although this class of substrates was alkynylated successfully, a lower enantioselectivity was observed (entries 5-7).

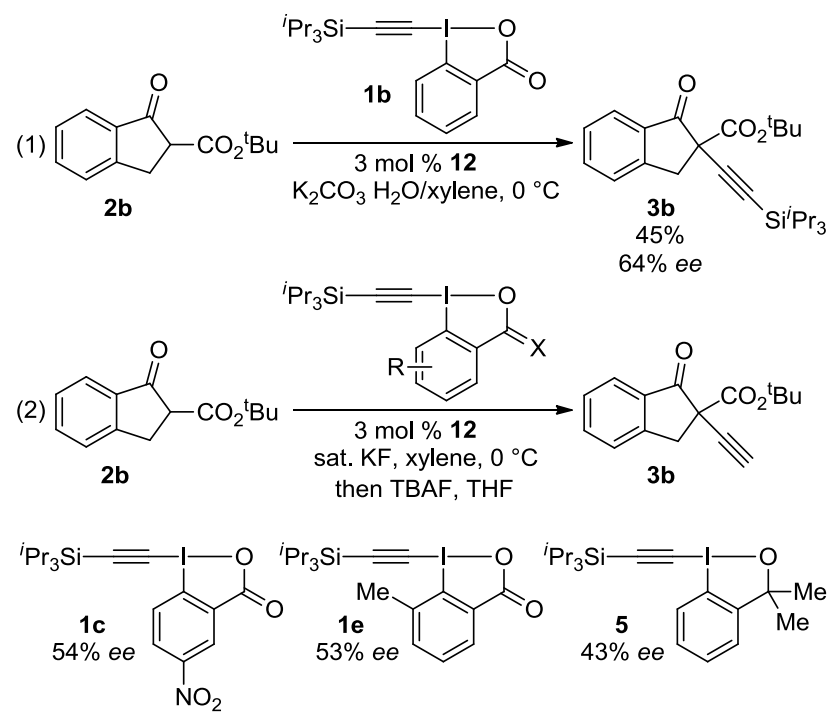

Scheme 6. Influence of reagent structure on enantioselectivity.

Throughout our work, asymmetric induction was never observed using alkynyliodonium salts instead of benziodoxolone reagents. At the first glance, this appears highly surprising if the reaction would simply proceed via direct $\mathrm{C}-\mathrm{C}$ bond formation between the catalyst-bound enolate and the hypervalent iodine reagent. However, Olofsson and co-workers have made recently a similar observation in the case of aryliodonium salts: no enantioselectivity could be achieved using different phase-transfer catalysts. ${ }^{[19]}$ To rationalize this result, they proposed that the first step was attack of the oxygen of the enolate on the iodine atom of the hypervalent iodine reagent, which led to decoordination of the catalyst. Logically, no asymmetric induction was possible anymore for C-C bond formation. This result was confirmed by calculation.

Transferred to the case of alkynylation, a similar mechanism would indeed allow rationalizing our observations (Scheme 7). Reaction of alkynyliodonium salt $\mathbf{4 a}$ with fluoride would lead to a fast desilylation and deprotonation of keto-ester $\mathbf{2 b}$ to form reagent $\mathbf{4 b}$ and the enolate intermediate $\mathbf{I}$. Attack of the oxygen on the iodine will then give neutral intermediate II and release the catalyst. C-C bond formation would most probably proceed via conjugate addition to give carbene III, as has been demonstrated by Ochiai and co-workers. ${ }^{[20]}$ Finally, 1,2-hydrogen shift would lead to racemic product $\mathbf{3 b}$. In the case of neutral TMS-EBX (1a), the formation of desilylated reagent $1 \mathrm{~g}$ had been indeed observed by NMR. ${ }^{[9]}$ Attack of the oxygen would then lead to intermediate $\mathbf{V}$. In contrast to II, the chiral catalyst would remain bound to the substrate, and asymmetric induction during the subsequent $\mathrm{C}-\mathrm{C}$ bond formation would become possible, albeit difficult. Intermediate $\mathbf{V}$ can then react intramolecularly to give carboxylate salt VI and carbene intermediate III. In this case, the final 1,2-shift could indeed be supported by a C13labelling experiment. ${ }^{[9]}$ This fundamental difference between alkynyliodonium and benziodoxolone reagents makes the latter superior for the design of new enantioselective methods.

Table 4. Asymmetric alkynylation of cyclic $\beta$-keto esters and amides.
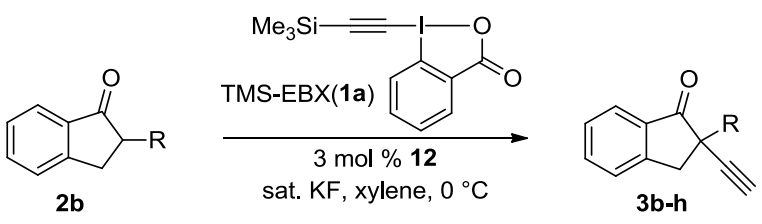

\begin{tabular}{|c|c|c|c|}
\hline Entry & $\mathrm{R}$ & Yield $^{[\mathrm{a}]}$ & ee \\
\hline 1 & & $70 \%$ & $65 \%$ \\
\hline 2 & & $83 \%$ & $79 \%$ \\
\hline 3 & & $99 \%$ & $55 \%$ \\
\hline 4 & & $84 \%$ & $27 \%$ \\
\hline 5 & & $57 \%$ & $16 \%$ \\
\hline 6 & & $93 \%$ & $30 \%$ \\
\hline 7 & & $51 \%$ & $55 \%$ \\
\hline
\end{tabular}

[a] General procedure: $0.20 \mathrm{mmol}$ substrate, 1.3 equiv. TMS-EBX (1a), catalyst 12 ( 0.03 equiv), sat. KF solution $(1 \mathrm{~mL})$, xylene $(2 \mathrm{~mL}), 0{ }^{\circ} \mathrm{C}$ to $23^{\circ} \mathrm{C}$. 


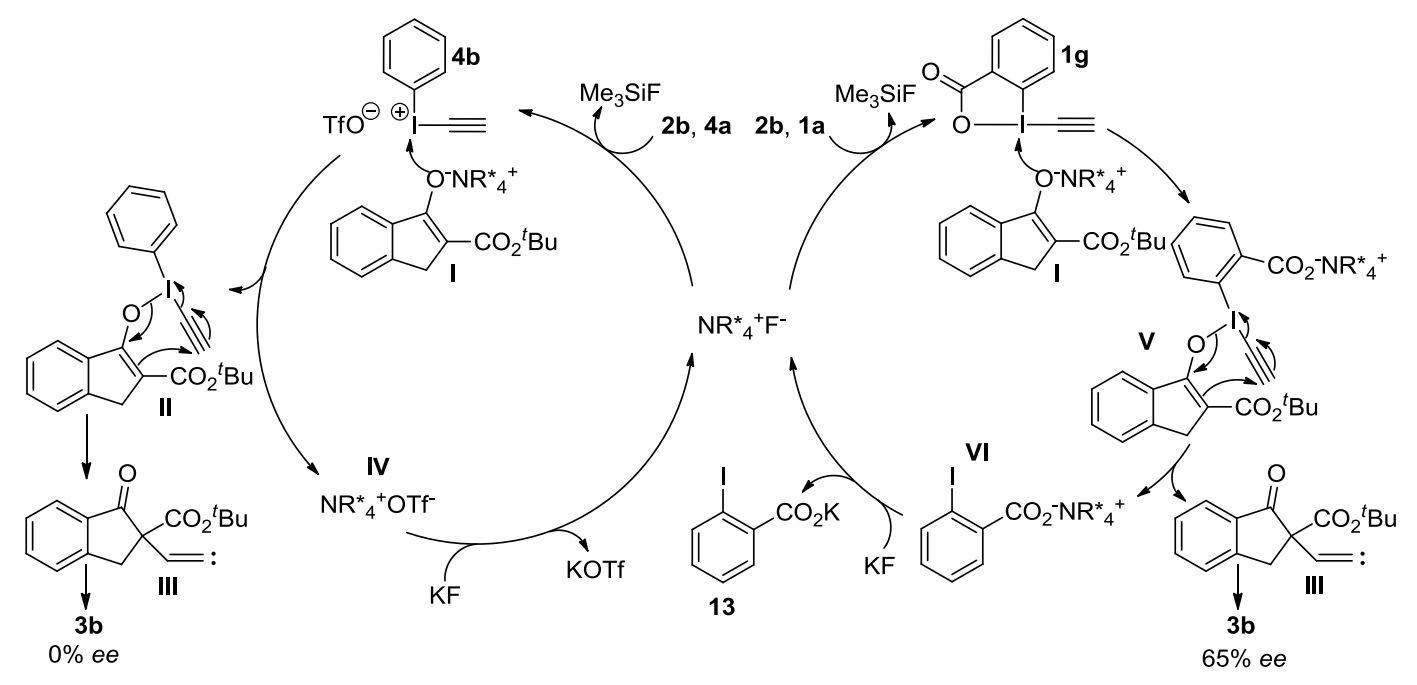

Scheme 7. Working model for the catalytic cycle with alkynyliodonium salt $\mathbf{4 a}$ and benziodoxolone $\mathbf{1 a}$.

\section{Conclusion}

In this work, we have studied the electrophilic alkynylation of cyclic keto-esters and amides with benziodoxolone reagents more in details. DSC studies have given a deeper insight into the safety profile of TMS-EBX (1a) and TIPS-EBX (1b). As both reagent showed a strong exothermic decomposition, adequate care has to be taken when manipulating them. Acetylene transfer could be extended to aromatic alkynes. A broad range of benziodoxol(on)e reagents could be used in the reaction.

Based on our preliminary results using cinchonabased phase-transfer catalysts, the enantioselective alkynylation of cyclic keto-esters was further improved. Maruoka's binaphthyl-derived ammonium salt 12 was identified as a better catalyst for asymmetric induction, and up to $79 \%$ ee could be obtained with an indanone-derived keto-ester, which is the highest enantioselectivity reported for this type of reactions. Throughout this work, asymmetric induction could be observed only in the case of benziodoxolone reagents, demonstrating their superiority over conventional alkynyliodonium salts. The deeper understanding gained about the factors leading to higher asymmetric induction will be very useful in the future to develop a truly general and highly enantioselective alkynylation method.

\section{Experimental Section}

\section{General Remarks}

See Supporting Information for general experimental details as well as procedures for the preparation and characterization of all precursors and products.

\section{Alkynylation Reaction}

General procedure for alkynylation using TBAF: A solution of substrate (1.0 equiv) and alkynyl benziodoxolone reagent (1.3 equiv) in dried THF $(60 \mathrm{mM})$ was stirred at $-78{ }^{\circ} \mathrm{C}$ for $5 \mathrm{~min}$ under nitrogen. After this period of time, TBAF (1 $\mathrm{M}$ in THF, 1.3 equiv) was added and the mixture was vigorously stirred at $-78{ }^{\circ} \mathrm{C}$. The reaction was monitored by TLC analysis (PET/EtOAc, 4:1, $\mathrm{UV}$ and $p$-anisaldehyde) and was complete at $-78{ }^{\circ} \mathrm{C}$ in the indicated time, or was slowly let to warm up to $10{ }^{\circ} \mathrm{C}$ during the indicated time. The reaction mixture was quenched with deactivated silica gel and the solvent was evaporated under reduced pressure. The product was purified via flash chromatography $\left(\mathrm{SiO}_{2}, \mathrm{Hexane} / \mathrm{EtOAc}\right)$ with the indicated solvent ratio.

General procedure for catalytic phase-transfer alkynylation: A solution of saturated base solution $(0.2 \mathrm{M})$ was added to a solution of phase-transfer catalyst (10 mol \%) and alkynyl benziodoxolone reagent (1.3 equiv) in toluene $(50 \mathrm{mM})$. The mixture was stirred at $0{ }^{\circ} \mathrm{C}$ for 5 min under nitrogen. After this period of time, the substrate (1.0 equiv) was added and the biphasic mixture was vigorously stirred at $0{ }^{\circ} \mathrm{C}$. The reaction was monitored by TLC analysis (PET/EtOAc 4:1, UV and $p$-anisaldehyde). After the indicated time, the reaction mixture was quenched with water and the aqueous layer was extracted with $\mathrm{CH}_{2} \mathrm{Cl}_{2}$. The combined organic layers were recollected, dried over $\mathrm{MgSO}_{4}$, filtered and concentrated under reduced pressure. The crude product was purified via silica gel flash chromatography $\left(\mathrm{SiO}_{2}, \mathrm{Hexane} / \mathrm{EtOAc}\right)$ with the indicated solvent ratio.

General procedure for catalytic phase-transfer alkynylation using Maruoka catalyst: A saturated base solution $(0.2 \mathrm{M})$ was added to a solution of phase-transfer catalyst (3 mol \%) and alkynyl benziodoxolone reagent (1.3 equiv) in xylene $(50 \mathrm{mM})$. The mixture was stirred at $0{ }^{\circ} \mathrm{C}$ for $5 \mathrm{~min}$ under nitrogen. After this period of time, the substrate (1.0 equiv) was added and the biphasic mixture was vigorously stirred at $0{ }^{\circ} \mathrm{C}$. The reaction was monitored by TLC analysis (PET/EtOAc 4:1, UV and p- 
anisaldehyde) and was complete at $0{ }^{\circ} \mathrm{C}$ in the indicated time, or was slowly let to warm up to $10{ }^{\circ} \mathrm{C}$ during the indicated time. The reaction mixture was quenched with water and the aqueous layer extracted with $\mathrm{CH}_{2} \mathrm{Cl}_{2}$. The combined organic layers were recollected, dried over $\mathrm{MgSO}_{4}$, filtered and concentrated under reduced pressure. The crude product was purified via silica gel flash chromatography $\left(\mathrm{SiO}_{2}, \mathrm{Hexane} / \mathrm{EtOAc}\right)$ with the indicated solvent ratio.

\section{1-[(Trimethylsilyl)ethynyl]-1,2-benziodoxol-3(1H)-one} (TMS-EBX, 1a): Trimethylsilyl triflate $(5.54 \mathrm{~mL}, 30.7$ mmol, 1.1 equiv) was added to a suspension of 2iodosylbenzoic acid (7.36 g, $28.0 \mathrm{mmol}, 1$ equiv) in $\mathrm{CH}_{2} \mathrm{Cl}_{2}(85 \mathrm{~mL})$ at $\mathrm{RT}$. The resulting yellow mixture was stirred for $1 \mathrm{~h}$, followed by the dropwise addition of bis(trimethylsilyl)acetylene $(6.98 \mathrm{~mL}, 30.7 \mathrm{mmol}, 1.1$ equiv). The resulting suspension was stirred for $6 \mathrm{~h}$ at RT, during this time a white solid was formed. A saturated solution of $\mathrm{NaHCO}_{3}$ was then added and the mixture was stirred vigorously until completely solubilization of the white solid. The two layers were separated and the combined organic extracts were washed with sat. $\mathrm{NaHCO}_{3}$, dried over $\mathrm{MgSO}_{4}$, filtered and evaporated under reduced pressure. Recrystallization from acetonitrile $(5 \mathrm{~mL})$ afforded $1 \mathbf{a}(7.17 \mathrm{~g}, 20.8 \mathrm{mmol}, 74 \%)$ as a colorless solid.

Mp (Dec.): $143-145{ }^{\circ} \mathrm{C} .{ }^{1} \mathrm{H}$ NMR (400 MHz, $\left.\mathrm{CDCl}_{3}\right) \delta$ $8.42(\mathrm{dd}, J=6.4,1.9 \mathrm{~Hz}, 1 \mathrm{H} ; \mathrm{ArH}), 8.19$ (m, $1 \mathrm{H} ; \mathrm{ArH})$, 7.78 (m, $2 \mathrm{H}$; ArH), 0.32 (s, $\left.9 \mathrm{H} ; \mathrm{Si}\left(\mathrm{CH}_{3}\right)\right) .{ }^{13} \mathrm{C}$ NMR (100 $\left.\mathrm{MHz}, \mathrm{CDCl}_{3}\right) \delta 166.4,134.9,132.6,131.7,131.4,126.1$, 117.2, 115.4, 64.2, -0.5. IR v 3389 (w), 2967 (w), 1617 (s), 1609 (s), 1562 (m), 1440 (w), 1350 (m), 1304 (w), 1254 (w), $1246(\mathrm{w}), 1112(\mathrm{w}), 1008(\mathrm{w}), 852(\mathrm{~s}), 746(\mathrm{~m}), 698$ (m), $639(\mathrm{~m})$.

\section{Acknowledgements}

EPFL and SNF (grant number 200021_119810) are acknowledged for financial support. Dr. Régis Mondière is acknowledged for the DSC studies on EBX reagents.

\section{References}

[1] F. Diederich, P. J. Stang, R. R. Tykwinsky, Acetylene Chemistry: Chemistry, Biology, and Material Science; Wiley-VCH: Weinheim, 2005.

[2] a) F. Alonso, I. P. Beletskaya, M. Yus, Chem. Rev. 2004, 104, 3079; b) E. Jimenez-Nunez, A. M. Echavarren, Chem. Commun. 2007, 333; c) J. F. Lutz, Angew. Chem., Int. Ed. 2007, 46, 1018; d) S. M. Abu Sohel, R. S. Liu, Chem. Soc. Rev. 2009, 38, 2269.

[3] B. M. Trost, A. H. Weiss, Adv. Synth. Catal. 2009, 351, 963.

[4] J. P. Brand, J. Waser, Chem. Soc. Rev. 2012, 41, 4165.

[5] A. S. Kende, P. Fludzinski, J. H. Hill, W. Swenson, J. Clardy, J. Am. Chem. Soc. 1984, 106, 3551.

[6] a) M. G. Moloney, J. T. Pinhey, E. G. Roche, J. Chem. Soc. Perkin Trans. 1 1989, 333; b) C. J. Parkinson, T. W. Hambley, J. T. Pinhey, J. Chem. Soc. Perkin Trans. 1 1997, 1465.

[7] a) M. Ochiai, T. Ito, Y. Takaoka, Y. Masaki, M. Kunishima, S. Tani, Y. Nagao, J. Chem. Soc. Chem.
Commun. 1990, 118; b) M. D. Bachi, N. Barner, C. M. Crittell, P. J. Stang, B. L. Williamson, J. Org. Chem. 1991, 56, 3912; c) M. D. Bachi, N. Barner, P. J. Stang, B. L. Williamson, J. Org. Chem. 1993, 58, 7923.

[8] T. B. Poulsen, L. Bernardi, J. Aleman, J. Overgaard, K. A. Jorgensen, J. Am. Chem. Soc. 2007, 129, 441.

[9] D. Fernandez Gonzalez, J. P. Brand, J. Waser, Chem. Eur. J. 2010, 16, 9457.

[10] a) J. P. Brand, J. Charpentier, J. Waser, Angew. Chem., Int. Ed. 2009, 48, 9346; b) S. Nicolai, C. Piemontesi, J. Waser, Angew. Chem., Int. Ed. 2011, 50, 4680; c) Y. Ohta, Y. Tokimizu, S. Oishi, N. Fujii, H. Ohno, Org. Lett. 2010, 12, 3963; d) H. Shi, L. C. Fang, C. H. Tan, L. L. Shi, W. B. Zhang, C. C. Li, T. P. Luo, Z. Yang, J. Am. Chem. Soc. 2011, 133, 14944; e) X. S. Liu, Z. T. Wang, X. M. Cheng, C. Z. Li, J. Am. Chem. Soc. 2012, 134, 14330. f) T. Aubineau, J. Cossy, Chem. Commun. 2013, 49, 3303; Review: g) J. P. Brand, D. Fernandez Gonzalez, S. Nicolai, J. Waser, Chem. Commun. 2011, 47, 102; Improved synthesis: h) M. J. Bouma, B. Olofsson, Chem. Eur. J. 2012, 18, 14242.

[11] M. Kamlar, P. Putaj, J. Veselý, Tetrahedron Lett. 2013, 54, 2097.

[12] CCDC-863343 contains the supplementary crystallographic data for this paper. These data can be obtained free of charge from The Cambridge Crystallographic Data Centre via www.ccdc.cam.ac.uk/data_request/cif.

[13] P. J. Stang, A. M. Arif, C. M. Crittell, Angew. Chem., Int. Ed.. 1990, 29, 2.

[14] P. J. Stang, M. Boehshar, H. Wingert, T. Kitamura, J. Am. Chem. Soc. 1988, 110, 3272.

[15] See supporting information for further details. For a similar recent study on Togni's reagent, a benziodoxole reagent used for trifluoromethylation, see: $\mathrm{N}$. Fiederling, J. Haller, H. Schramm, Org. Proc. Res. Dev. 2013, 17, 318 .

[16] V. V. Zhdankin, C. J. Kuehl, A. P. Krasutsky, J. T. Bolz, A. J. Simonsen, J. Org. Chem. 1996, 61, 6547.

[17] a) D. Uraguchi, Y. Ueki, T. Ooi, J. Am. Chem. Soc. 2008, 130, 14088; b) D. Uraguchi, T. Ito, T. Ooi, J. Am. Chem. Soc. 2009, 131, 3836.

[18] a) T. Ooi, T. Miki, M. Taniguchi, M. Shiraishi, M. Takeuchi, K. Maruoka, Angew. Chem., Int. Ed. 2003, 42, 3796; b) T. Hashimoto, K. Sakata, K. Maruoka, Angew. Chem., Int. Ed. 2009, 48, 5014.

[19] P. O. Norrby, T. B. Petersen, M. Bielawski, B. Olofsson, Chem. Eur. J. 2010, 16, 8251.

[20] M. Ochiai, M. Kunishima, Y. Nagao, K. Fuji, M. Shiro, E. Fujita, J. Am. Chem. Soc. 1986, 108, 8281. 


\section{UPDATE}

Ethynyl BenziodoXolones (EBX) as Reagents for the Ethynylation of Stabilized Enolates

Adv. Synth. Catal. Year, Volume, Page - Page

Davinia Fernández González, ${ }^{\mathrm{a}}$ Jonathan P. Brand, ${ }^{\mathrm{a}}$ Régis Mondière, ${ }^{\mathrm{b}}$ and Jérôme Waser*

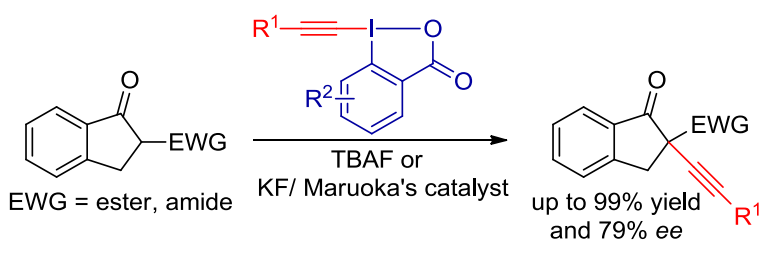




\section{Ethynyl BenziodoXolones (EBX) as Reagents for the Ethynylation of Stabilized Enolates}

Davinia Fernández González, ${ }^{a}$ Jonathan P. Brand, ${ }^{a}$ Régis Mondière, ${ }^{b}$ and Jérôme Waser $^{a *}$

a Laboratory of Catalysis and Organic Synthesis, Ecole Polytechnique Fédérale de Lausanne, CH-1015, Switzerland. Fax: +41 (0)21 69397 00; Phone: +41 (0)21 69393 88; E-mail: jerome.waser@epfl.ch

b Syngenta Crop Protection Münchwilen AG, Research Chemistry, Schaffhauserstrasse, CH-4332 Stein, Switzerland

\section{Supporting Information}

\section{Table of Content}

65 pages

1. General Methods $\quad$ S2

2. Preparation of Reagents, Substrates and Catalysts S3

3. Alkynylation Reactions $\quad$ S26

4. Spectra of New Compounds $\quad$ S35 


\section{General Methods}

All reactions were carried out in oven dried glassware under an atmosphere of nitrogen, unless stated otherwise. For quantitative flash chromatography technical grade solvents were used. For flash chromatography for analysis, HPLC grade solvents from Sigma-Aldrich were used. THF, $\mathrm{Et}_{2} \mathrm{O}, \mathrm{CH}_{3} \mathrm{CN}$, toluene, hexane and $\mathrm{CH}_{2} \mathrm{Cl}_{2}$ were dried by passage over activated alumina under nitrogen atmosphere $\left(\mathrm{H}_{2} \mathrm{O}\right.$ content $<10 \mathrm{ppm}$, Karl-Fischer titration $)$. $\mathrm{NEt}_{3}$ and pyridine were distilled under nitrogen from $\mathrm{KOH}$. All chemicals were purchased from Acros, Aldrich, Fluka, VWR, Aplichem, Maybrige, TCI or Merck and used as such unless stated otherwise. Chromatographic purification was performed as flash chromatography using Macherey-Nagel silica 40-63, $60 \AA$, using the solvents indicated as eluent with 0.1-0.5 bar pressure. Macherey -Nagel silica 40-63, $60 \AA$ was deactivated using $\mathrm{EtN}_{3}$ (2\% v/v) in the solvents indicated as eluent and the solvents were removed under reduce pressure. TLC was performed on Merck silica gel $60 \mathrm{~F}_{254}$ TLC glass plates or aluminum plates and visualized with UV light, permanganate stain, CAN stain or Anisaldehyde stain. Melting points were measured on a Büchi B-540 melting point apparatus using open glass capillaries, the data is uncorrected. DSCs were measured on a Mettler Toledo DSC822e using high pressure gold plated $40 \mu \mathrm{L}$ crucibles. Approximately $8 \mathrm{mg}$ of substance were precisely weighted to the hundredth of milligram on a Mettler Toledo AX105 Deltarange® balance and inserted into the DSC crucible. The sealed DSC crucible weight was measured with the same precision and recorded. The DSC crucible was then placed into the DSC oven and subjected to a temperature ramp from $30^{\circ} \mathrm{C}$ to $350^{\circ} \mathrm{C}$ with a gradient of $4^{\circ} \mathrm{C}$ per minute. Integrations of endotherms and exotherms were performed using a linear baseline. After DSC measurement, the DSC crucible weight was checked to ensure that no weight loss had occurred during the DSC measurement. ${ }^{1} \mathrm{H}-\mathrm{NMR}$ spectra were recorded on a Brucker DPX-400 $400 \mathrm{MHz}$ or Brucker AV-400 400MHZ spectrometer in chloroform-d, $\mathrm{CD}_{2} \mathrm{Cl}_{2}-\mathrm{d}_{2}$, DMSO- $\mathrm{d}_{6}$ or $\mathrm{CD}_{3} \mathrm{OD}$, all signals are reported in ppm with the internal chloroform signal at $7.26 \mathrm{ppm}$, the internal $\mathrm{CD}_{2} \mathrm{Cl}_{2}$ signal at $5.33 \mathrm{ppm}$, the internal DMSO signal at $2.50 \mathrm{ppm}$ or the internal methanol signal at $3.30 \mathrm{ppm}$ as standard. The data is being reported as $(\mathrm{s}=$ singlet, $\mathrm{d}=$ doublet, $\mathrm{t}=$ triplet, $\mathrm{q}=$ quadruplet, $\mathrm{qi}=$ quintet, $\mathrm{m}=$ multiplet or unresolved, $\mathrm{br}=$ broad signal, $\mathrm{app}=$ apparent, coupling constant(s) in $\mathrm{Hz}$, integration; interpretation) $.{ }^{13} \mathrm{C}-\mathrm{NMR}$ spectra were recorded with ${ }^{1} \mathrm{H}$-decoupling on a Brucker DPX-400 $100 \mathrm{MHz}$ or Brucker AV-400 400MHZ spectrometer in chloroform-d, $\mathrm{CD}_{2} \mathrm{Cl}_{2}-\mathrm{d}_{2}$, DMSO- $\mathrm{d}_{6}$ or $\mathrm{CD}_{3} \mathrm{OD}$, all signals are reported in ppm with the internal chloroform signal at $77.0 \mathrm{ppm}$, the internal $\mathrm{CD}_{2} \mathrm{Cl}_{2}$ signal at $53.0 \mathrm{ppm}$, the internal DMSO signal at $39.5 \mathrm{ppm}$ or the internal methanol signal at $49.0 \mathrm{ppm}$ as standard. Infrared spectra were recorded on a JASCO FT-IR B4100 spectrophotometer with an ATR PRO410-S and a ZnSe prism and are reported as $\mathrm{cm}^{-1}(\mathrm{w}=$ weak, $\mathrm{m}=$ medium, $\mathrm{s}=$ strong, br = broad). Gas chromatographic and low resolution mass spectrometric measurements were performed on a Perkin-Elmer Clarus 600 gas chromatographer and mass spectrometer using a Perkin-Elmer Elite fused silica column (length: $30 \mathrm{~m}$, diameter: $0.32 \mathrm{~mm}$ ) and Helium as carrier gas. High resolution mass spectrometric measurements were performed by the mass spectrometry service of ISIC at the EPFL on a MICROMASS (ESI) Q-TOF Ultima API. Elemental analysis was performed at the elemental analysis of ISIC at EPFL. HPLC measurements were done on a JASCO HPLC system with an AS 2055 Autosampler, a PV 2089 Pump, a UV 2075 detector and a SEDEX 85 (SEDERE) detector using a CHIRALPAC IC column from DAICEL Chemical industries Ltd. HPLC grade solvents from Sigma-Aldrich were used. 
Caution: Hypervalent iodine reagents are high energy compounds. Although no problem has ever been encountered in this work, adequate care (safety shield) has to be taken, especially during large scale synthesis of reagents.

\section{Preparation of Reagents, Substrates and Catalysts}

\subsection{Preparation of Reagents}

\section{1-Hydroxy-1,2-benziodoxol-3-(1H)-one (15)}

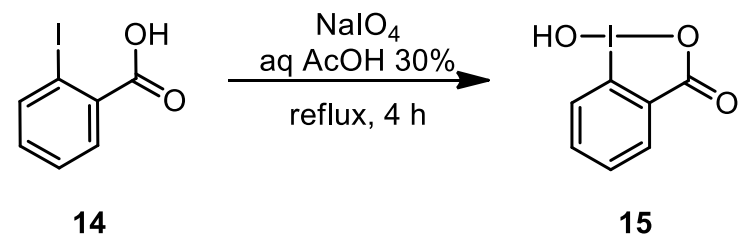

Following the reported procedure, ${ }^{[1]} \mathrm{NaIO}_{4}(7.24 \mathrm{~g}, 33.8 \mathrm{mmol}, 1.05$ equiv) and 2iodobenzoic acid (14) (8.00 g, $32.2 \mathrm{mmol}, 1.00$ equiv) were suspended in $30 \%$ (v:v) aq. $\mathrm{AcOH}(48 \mathrm{~mL})$. The mixture was vigorously stirred and refluxed for $4 \mathrm{~h}$. The reaction mixture was then diluted with cold water $(180 \mathrm{~mL})$ and allowed to cool to RT, protecting it from light. After $1 \mathrm{~h}$, the crude product was collected by filtration, washed on the filter with ice water (3 x $20 \mathrm{~mL})$ and acetone $(3 \times 20 \mathrm{~mL})$, and air-dried in the dark to give the pure product $\mathbf{1 5}(8.3$ $\mathrm{g}, 31 \mathrm{mmol}, 98 \%$ ) as a colorless solid.

${ }^{1} \mathrm{H}$ NMR $\left(400 \mathrm{MHz},\left(\mathrm{CD}_{3}\right)_{2} \mathrm{SO}\right) \delta 8.02(\mathrm{dd}, J=7.7,1.4 \mathrm{~Hz}, 1 \mathrm{H} ; \mathrm{Ar} H), 7.97(\mathrm{~m}, 1 \mathrm{H}$; $\operatorname{Ar} H), 7.85(\mathrm{dd}, J=8.2,0.7 \mathrm{~Hz}, 1 \mathrm{H} ; \mathrm{Ar} H), 7.71(\mathrm{td}, J=7.6,1.2 \mathrm{~Hz}, 1 \mathrm{H} ; \mathrm{Ar} H) .{ }^{13} \mathrm{C} \mathrm{NMR}$ $\left(100 \mathrm{MHz},\left(\mathrm{CD}_{3}\right)_{2} \mathrm{SO}\right) \delta 167.7,134.5,131.5,131.1,130.4,126.3,120.4$. IR $v 3083(\mathrm{w}), 3060$ (w), $2867(\mathrm{w}), 2402(\mathrm{w}), 1601(\mathrm{~m}), 1585(\mathrm{~m}), 1564(\mathrm{~m}), 1440(\mathrm{~m}), 1338(\mathrm{~s}), 1302(\mathrm{~m}), 1148$ (m), $1018(\mathrm{w}), 834(\mathrm{~m}), 798(\mathrm{w}), 740$ (s), $694(\mathrm{~s}), 674(\mathrm{~m}), 649(\mathrm{~m})$. The characterization data corresponded to the reported values. ${ }^{[1]}$

\section{1-[(Trimethylsilyl)ethynyl]-1,2-benziodoxol-3(1H)-one (1a)}<smiles>O=C1OI(O)c2ccccc21</smiles>

15

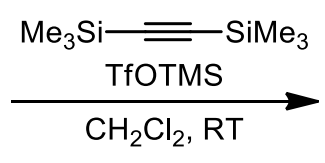

$\mathrm{CH}_{2} \mathrm{Cl}_{2}, \mathrm{RT}$

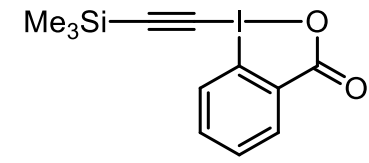

$1 \mathrm{a}$

Following a slight modification of the reported procedure, ${ }^{[2]}$ trimethylsilyl triflate $(5.54$ $\mathrm{mL}, 30.7 \mathrm{mmol}, 1.1$ equiv) was added to a suspension of 2-iodosylbenzoic acid (15) (7.36 g, 28.0 mmol, 1 equiv) in $\mathrm{CH}_{2} \mathrm{Cl}_{2}(85 \mathrm{~mL})$ at RT. The resulting yellow mixture was stirred for 1 $\mathrm{h}$, followed by the dropwise addition of bis(trimethylsilyl)acetylene $(6.98 \mathrm{~mL}, 30.7 \mathrm{mmol}, 1.1$ equiv). The resulting suspension was stirred for $6 \mathrm{~h}$ at RT, during this time a white solid was formed. A saturated solution of $\mathrm{NaHCO}_{3}$ was then added and the mixture was stirred vigorously until completely solubilization of the white solid. The two layers were separated and the combined organic extracts were washed with sat. $\mathrm{NaHCO}_{3}$, dried over $\mathrm{MgSO}_{4}$, filtered and evaporated under reduce pressure. Recrystallization from acetonitrile $(5 \mathrm{~mL})$

[1] L. Kraszkiewicz, L. Skulski, Arkivoc. 2003, 6, 120.

[2] V. V. Zhdankin, C. J. Kuehl, A. P. Krasutsky, J. T. Bolz, A. J. Simonsen, J. Org. Chem. 1996, 61, 6547. 
afforded $1 \mathrm{a}(7.17 \mathrm{~g}, 20.8 \mathrm{mmol}, 74 \%)$ as a colorless solid. The crystal structure of $\mathbf{1 a}$ has been deposited at the Cambridge Crystallographic Data Centre and allocated the deposition number: CCDC 863343.

Mp (Dec.): $143-145{ }^{\circ} \mathrm{C} .{ }^{1} \mathrm{H}$ NMR $\left(400 \mathrm{MHz}, \mathrm{CDCl}_{3}\right) \delta 8.42(\mathrm{dd}, J=6.4,1.9 \mathrm{~Hz}, 1 \mathrm{H}$; $\operatorname{ArH}), 8.19$ (m, $1 \mathrm{H} ; \mathrm{ArH}), 7.78(\mathrm{~m}, 2 \mathrm{H} ; \mathrm{ArH}), 0.32$ (s, $\left.9 \mathrm{H} ; \mathrm{Si}\left(\mathrm{CH}_{3}\right)\right) .{ }^{13} \mathrm{C}$ NMR $(100 \mathrm{MHz}$, $\left.\mathrm{CDCl}_{3}\right) \delta 166.4,134.9,132.6,131.7,131.4,126.1,117.2,115.4,64.2,-0.5 . \mathrm{IR} \vee 3389(\mathrm{w})$, 2967 (w), 1617 (s), 1609 (s), 1562 (m), 1440 (w), 1350 (m), 1304 (w), 1254 (w), 1246 (w), $1112(\mathrm{w}), 1008(\mathrm{w}), 852(\mathrm{~s}), 746(\mathrm{~m}), 698(\mathrm{~m}), 639(\mathrm{~m})$. The characterization data corresponded to the reported values. ${ }^{[2]}$

\section{Triiso-propylsilyl trimethylsilylacetylene (17)}

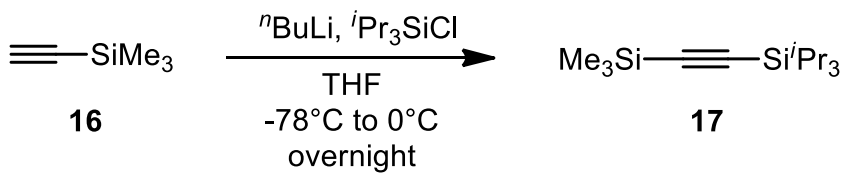

Following a reported procedure, ${ }^{[3]} n$-butyllithium $(2.5 \mathrm{M}$ in hexanes, $12.0 \mathrm{~mL}, 29.9 \mathrm{mmol}$, 0.98 equiv) was added dropwise to a stirred solution of ethynyltrimethylsilane (16) $(3.0 \mathrm{~g}, 30$ mmol, 1.0 equiv) in THF ( $48 \mathrm{~mL}$ ) at $-78{ }^{\circ} \mathrm{C}$. The mixture was then warmed to $0{ }^{\circ} \mathrm{C}$ and stirred for $5 \mathrm{~min}$. The mixture was then cooled back to $-78{ }^{\circ} \mathrm{C}$ and chlorotriiso-propylsilane ( $6.4 \mathrm{~mL}, 30 \mathrm{mmol}, 1.0$ equiv) was added dropwise. The mixture was then allowed to warm to room temperature and stirred overnight. A saturated solution of ammonium chloride $(40 \mathrm{~mL})$ was added, and the reaction mixture was extracted with diethyl ether $(2 \times 60 \mathrm{~mL})$. The organic layer was washed with water and brine, then dried over $\mathrm{MgSO}_{4}$, filtered and concentrated under reduced pressure to obtain a colorless liquid which was further purified by Kugelrohr distillation $\left(\mathrm{bp}=56-57{ }^{\circ} \mathrm{C}, \mathrm{p}=0.25 \mathrm{mmHg}\right)$ to yield $17(7.16 \mathrm{~g}, 28.0 \mathrm{mmol}, 92 \%$ yield) as a colorless liquid.

${ }^{1} \mathrm{H}$ NMR $\left(400 \mathrm{MHz}, \mathrm{CDCl}_{3}\right) \delta 1.08\left(\mathrm{~m}, 21 \mathrm{H} ; \mathrm{Si}\left(\mathrm{CH}\left(\mathrm{CH}_{3}\right)_{2}\right)_{3}\right), 0.18\left(\mathrm{~s}, 9 \mathrm{H} ; \mathrm{Si}\left(\mathrm{CH}_{3}\right)\right)$. IR v 2959 (m), 2944 (m), 2896 (w), 2867 (m), 1464 (w), 1385 (w), 1250 (m), 996 (w), 842 (s), 764 $(\mathrm{s}), 675(\mathrm{~m}), 660(\mathrm{~m})$. The characterization data corresponded to the reported values. ${ }^{[3]}$

\section{1-[(Triiso-propylsilyl)ethynyl]-1,2-benziodoxol-3(1H)-one (1b)}<smiles>O=C1OI(O)c2ccccc21</smiles>

15

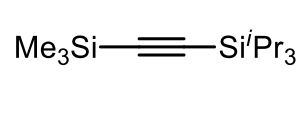

17

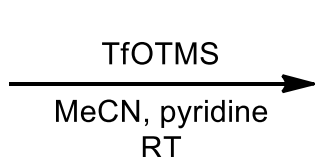

RT

Following a slight modification of the reported procedure, ${ }^{[2]}$ trimethylsilyltriflate $(3.6 \mathrm{~mL}$, $20 \mathrm{mmol}, 1.1$ equiv, freshly distilled) was added dropwise to a stirred solution of 2iodosylbenzoic acid (15) (4.7 g, $18 \mathrm{mmol}, 1.0$ equiv) in acetonitrile (140 $\mathrm{mL})$. (Trimethylsilyl)(triiso-propylsilyl)acetylene (17) $(5.0 \mathrm{~g}, 20 \mathrm{mmol}, 1.1$ equiv) was then added dropwise, followed, after $15 \mathrm{~min}$, by the addition of pyridine $(1.5 \mathrm{~mL}, 20 \mathrm{mmol}, 1.1$ equiv). The mixture was stirred $10 \mathrm{~min}$. The solvent was then removed under reduced pressure and the yellow crude oil was dissolved in dichloromethane $(50 \mathrm{~mL})$. The organic layer was

[3] C J. Helal, P. A. Magriotis, E. J. Corey, J. Am. Chem. Soc. 1996, 118, 10938. 
washed with $1 \mathrm{M} \mathrm{HCl}(50 \mathrm{~mL})$ and the aqueous layer was extracted with $\mathrm{CH}_{2} \mathrm{Cl}_{2}(50 \mathrm{~mL})$. The organic layers were combined, washed with a saturated solution of $\mathrm{NaHCO}_{3}(2 \times 50 \mathrm{~mL})$, dried over $\mathrm{MgSO}_{4}$, filtered and the solvent was evaporated under reduced pressure. Recrystallization from acetonitrile (ca $35 \mathrm{~mL})$ afforded $\mathbf{1 b}(6.3 \mathrm{~g}, 15 \mathrm{mmol}, 83 \%)$ as a colorless solid.

Mp (Dec.) $170-176{ }^{\circ} \mathrm{C} .{ }^{1} \mathrm{H}$ NMR $\left(400 \mathrm{MHz}, \mathrm{CDCl}_{3}\right) \delta 8.44(\mathrm{~m}, 1 \mathrm{H} ; \mathrm{Ar} H), 8.29(\mathrm{~m}, 1 \mathrm{H}$; $\mathrm{ArH}), 7.77(\mathrm{~m}, 2 \mathrm{H}$; $\mathrm{ArH}), 1.16\left(\mathrm{~m}, 21 \mathrm{H} ; \mathrm{Si}\left(\mathrm{CH}\left(\mathrm{CH}_{3}\right)_{2}\right)_{3}\right) .{ }^{13} \mathrm{C} \mathrm{NMR}\left(100 \mathrm{MHz}, \mathrm{CDCl}_{3}\right) \delta$ 166.4, 134.6, 132.3, 131.4, 131.4, 126.1, 115.6, 114.1, 64.6, 18.4, 11.1. IR v $2943(\mathrm{~m}), 2865$ (m), $1716(\mathrm{~m}), 1618(\mathrm{~m}), 1604(\mathrm{~s}), 1584(\mathrm{~m}), 1557(\mathrm{~m}), 1465(\mathrm{~m}), 1439$ (w), $1349(\mathrm{~m}), 1291$ (m), $1270(\mathrm{w}), 1244(\mathrm{~m}), 1140(\mathrm{~m}), 1016(\mathrm{~m}), 999(\mathrm{~m}), 883(\mathrm{~m}), 833(\mathrm{~m}), 742(\mathrm{~m}), 702(\mathrm{~s})$, $636(\mathrm{~m})$. The characterization data corresponded to the reported values. ${ }^{[2]}$

\section{Phenyl(trimethylsilylethynyl)iodonium triflate (4)}

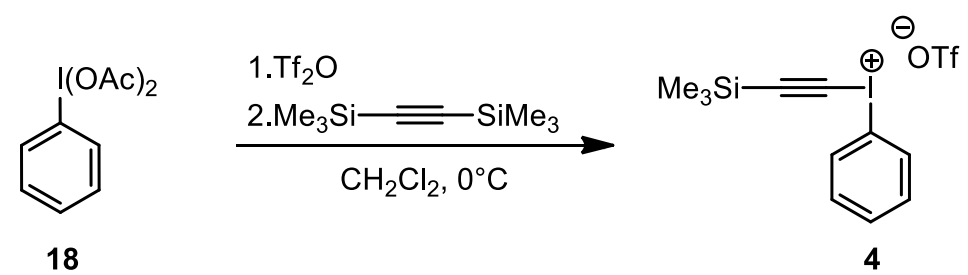

Following the reported procedure, ${ }^{[4]}$ phenyliodonium diacetate $(\mathbf{1 8})(1.6 \mathrm{~g}, 5.0 \mathrm{mmol}, 1.0$ equiv) was diluted with $\mathrm{CH}_{2} \mathrm{Cl}_{2}(5 \mathrm{~mL})$ in a two-neck flask and the mixture was stirred $5 \mathrm{~min}$. $\mathrm{Tf}_{2} \mathrm{O}\left(0.42 \mathrm{~mL}, 2.5 \mathrm{mmol}, 0.50\right.$ equiv) was added dropwise at $0{ }^{\circ} \mathrm{C}$ and the resulting yellow mixture was stirred $30 \mathrm{~min}$. Bis(trimethylsilyl)acetylene $(1.15 \mathrm{~mL}, 5.00 \mathrm{mmol}, 1.00$ equiv) was added. The mixture was then stirred $2 \mathrm{~h}$ and diethyl ether was added to precipitate the product. Filtration afforded $4(1.64 \mathrm{~g}, 3.60 \mathrm{mmol}, 73 \%)$ as colorless crystals.

Mp (Dec.) 139-145 ${ }^{\circ} \mathrm{C}$; Lit.: ${ }^{[4]} 143-146{ }^{\circ} \mathrm{C} .{ }^{1} \mathrm{H}$ NMR $\left(400 \mathrm{MHz}, \mathrm{CDCl}_{3}\right) \delta 8.07(\mathrm{~d}, J=8.3$ $\mathrm{Hz}, 2 \mathrm{H}$; $\mathrm{ArH}$ ), 7.66 (s, $1 \mathrm{H} ; \mathrm{ArH}), 7.55$ (m, $2 \mathrm{H} ; \mathrm{ArH}), 0.24$ (s, $\left.9 \mathrm{H} ; \mathrm{Si}\left(\mathrm{CH}_{3}\right)\right) .{ }^{13} \mathrm{C}$ NMR $(100$ $\left.\mathrm{MHz}, \mathrm{CDCl}_{3}\right) \delta 133.9,132.4,132.2,119.7\left(\mathrm{q},{ }^{1} J(\mathrm{C}, \mathrm{F})=319 \mathrm{~Hz}\right), 119.1,116.2,43.3,-1.1 . \mathrm{IR}$ $v 1448(\mathrm{w}), 1286(\mathrm{~m}), 1253(\mathrm{~m}), 1236(\mathrm{~s}), 1222(\mathrm{~s}), 1161(\mathrm{~m}), 1026(\mathrm{~s}), 988(\mathrm{w}), 863(\mathrm{~m}), 847$ $(\mathrm{s}), 742(\mathrm{w}), 714(\mathrm{~m}), 678(\mathrm{w}), 637(\mathrm{~s})$. The characterization data corresponded to the reported

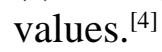

2-Iodosyl-5-nitrobenzoic acid and 2-iodosyl-3-nitrobenzoic acid (19, 20)<smiles>O=C(O)c1ccccc1I</smiles>

14

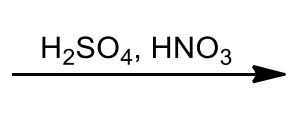

$$
\mathrm{O}_{2}
$$<smiles>O=Ic1ccc([N+](=O)[O-])cc1C(=O)O</smiles>

19<smiles>O=C(O)c1cccc([N+](=O)[O-])c1C(=O)O</smiles>

20

Following a reported procedure, ${ }^{[5]}$ fuming nitric acid $(3.3 \mathrm{~mL})$ was added to 2-iodobenzoic acid (14) $\left(5.0 \mathrm{~g}, 20 \mathrm{mmol}, 1.0\right.$ equiv) in concentrated $\mathrm{H}_{2} \mathrm{SO}_{4}(6.7 \mathrm{~mL})$. The reaction was equipped with a cooler and a nitrous vapor trap and was heated at $100{ }^{\circ} \mathrm{C}$ for $1 \mathrm{~h}$. The reaction mixture was then poured onto ice-water and filtered. The resulting solid was refluxed in water $(50 \mathrm{~mL})$ and filtered. A second crop of precipitate was filtered from the mother liquors. Both

[4] T. Kitamura, M. Kotani, Y. Fujiwara, Synthesis 1998, 1416.

[5] G. F. Morrison, J. Hooz, J. Org. Chem. 1970, 35, 1196. 
solids were combined, washed with acetone $(10 \mathrm{~mL})$ and dried under vacuum to afford 19 $(2.19 \mathrm{~g}, 7.10 \mathrm{mmol}, 36 \%)$. The mother liquors were reduced to one third and then kept at 4 ${ }^{\circ} \mathrm{C}$, the resulting precipitate was filtered, washed with acetone $(10 \mathrm{~mL})$ and dried under vacuum to afford 20 (630 $\mathrm{mg}, 2.04 \mathrm{mmol}, 10 \%)$.

19: ${ }^{1} \mathrm{H}$ NMR (400 MHz, DMSO) $\delta 8.73(\mathrm{dd}, J=8.8,2.6 \mathrm{~Hz}, 1 \mathrm{H}$; ArH), $8.58(\mathrm{~d}, J=2.4$ $\mathrm{Hz}, 1 \mathrm{H} ; \mathrm{ArH}$ ), 8.54 (br s, $1 \mathrm{H} ; \mathrm{OH}), 8.11$ (d, $J=8.8 \mathrm{~Hz}, 1 \mathrm{H}$; ArH).

20: ${ }^{1} \mathrm{H}$ NMR (400 MHz, DMSO) $\delta 7.92(\mathrm{dd}, J=7.9,1.5 \mathrm{~Hz}, 1 \mathrm{H} ; \operatorname{Ar} H), 7.79(\mathrm{~m}, 1 \mathrm{H}$; $\operatorname{ArH}), 7.67$ (m, $1 \mathrm{H} ; \mathrm{ArH})$.

\section{5-Nitro-1-[(triisopropylsilyl)ethynyl]-1,2-benziodoxol-3(1H)-one (1c)}<smiles>O=C1OI(O)c2ccc([N+](=O)[O-])cc21</smiles>

19

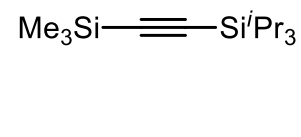

17

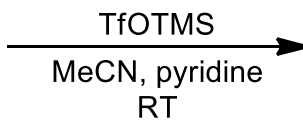

RT

Trimethylsilyltriflate $\left(646 \mu \mathrm{L}, 3.56 \mathrm{mmol}, 1.10\right.$ equiv, freshly distilled over $\left.\mathrm{CaH}_{2}\right)$ was added dropwise to a stirred solution of 2-iodosylbenzoic acid 19 (1.00 g, $3.23 \mathrm{mmol}, 1.00$ equiv) in acetonitrile $\left(15 \mathrm{~mL}\right.$ ) at $0{ }^{\circ} \mathrm{C}$. After $15 \mathrm{~min}$ at RT, (trimethylsilyl)(triisopropylsilyl)acetylene (17) (906 mg, $3.56 \mathrm{mmol}, 1.10$ equiv) was added dropwise, followed, after $30 \mathrm{~min}$, by the addition of pyridine $(290 \mu \mathrm{L}, 3.56 \mathrm{mmol}, 1.10$ equiv). The mixture was stirred $20 \mathrm{~min}$. The solvent was then removed under reduced pressure and the yellow crude oil was dissolved in dichloromethane $(25 \mathrm{~mL})$. The organic layer was washed with $1 \mathrm{M} \mathrm{HCl}(25$ $\mathrm{mL})$ and the aqueous layer was extracted with $\mathrm{CH}_{2} \mathrm{Cl}_{2}(25 \mathrm{~mL})$. The organic layers were combined, washed with a saturated solution of $\mathrm{NaHCO}_{3}(20 \mathrm{~mL})$, dried over $\mathrm{MgSO}_{4}$, filtered and the solvent was evaporated under reduced pressure. Recrystallization from acetonitrile ( $\mathrm{ca}$ $20 \mathrm{~mL}$ ) afforded 1c (960 mg, $2.02 \mathrm{mmol}, 63 \%)$ as a colorless solid.

$\mathrm{Mp}$ (Dec.) $198-206{ }^{\circ} \mathrm{C} .{ }^{1} \mathrm{H} \mathrm{NMR}\left(400 \mathrm{MHz}, \mathrm{CDCl}_{3}\right)(\mathrm{ca} 0.13 \mathrm{mmol} / \mathrm{ml}) \delta 9.20(\mathrm{~d}, J=2.6$ $\mathrm{Hz}, 1 \mathrm{H}$; ArH), 8.60 (ddd, $J=9.0,2.5,0.4 \mathrm{~Hz}, 1 \mathrm{H} ; \operatorname{Ar} H), 8.53$ (d, $J=8.9 \mathrm{~Hz}, 1 \mathrm{H}$; ArH), 1.30-1.14 (m, $21 \mathrm{H}$; $\left.\mathrm{Si}\left(\mathrm{CH}\left(\mathrm{CH}_{3}\right)_{2}\right)_{3}\right) .{ }^{13} \mathrm{C} \mathrm{NMR}\left(101 \mathrm{MHz}, \mathrm{CDCl}_{3}\right) \delta$ 166.4, 150.7, 134.4, 129.0, 128.2, 126.5, 122.7, 115.2, 63.1, 18.5, 11.3. IR 3094 (w), 2947 (w), 2869 (w), 2387 (w), $2361(\mathrm{w}), 2298$ (w), 1630 (s), $1571(\mathrm{w}), 1534$ (s), 1473 (w), 1415 (w), 1340 (s), 1272 (w), $1168(\mathrm{w}), 1074(\mathrm{w}), 930(\mathrm{w}), 907(\mathrm{w}), 825(\mathrm{~m}), 796(\mathrm{w}), 731(\mathrm{~s}), 712(\mathrm{~m}), 667$ (m), 632 (w). The characterization data corresponded to the reported values. ${ }^{[6]}$

\section{4,5-Dimethoxy-2-iodosylbenzoic acid (22)}<smiles>COc1cc(I)c(C(=O)O)cc1OC</smiles>

21

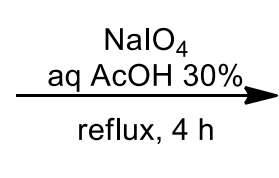

reflux, $4 \mathrm{~h}$<smiles>COc1cc2c(cc1OC)I(O)OC2=O</smiles>

22

[6] Ohta, Y.; Tokimizu, Y.; Oishi, S.; Fujii, N.; Ohno, H. Org. Lett. 2010, 12, 3963. 
Following the reported procedure, ${ }^{[1]} \mathrm{NaIO}_{4}(1.25 \mathrm{~g}, 5.84 \mathrm{mmol}, 1.05$ equiv) and 2-iodo4,5-dimethoxybenzoic acid (21) (1.71 g, $5.56 \mathrm{mmol}, 1.00$ equiv) were suspended in $30 \%$ (v:v) aq. $\mathrm{AcOH}(15 \mathrm{~mL})$. The mixture was vigorously stirred and refluxed for $4 \mathrm{~h}$. The reaction mixture was then diluted with cold water $(40 \mathrm{~mL})$ and allowed to cool to RT, protecting it from light. The crude product was collected by filtration, washed on the filter with ice water $(3 \times 4 \mathrm{~mL})$ and acetone $(3 \times 4 \mathrm{~mL})$, and air-dried in the dark to give the pure product $22(1.64 \mathrm{~g}$, $5.06 \mathrm{mmol}, 91 \%)$ as a colorless solid.

${ }^{1} \mathrm{H}$ NMR (400 MHz, DMSO) $\delta 7.45$ (s, $\left.1 \mathrm{H} ; \mathrm{ArH}\right), 7.23$ (s, $\left.1 \mathrm{H} ; \mathrm{ArH}\right), 3.88$ (d, J=0.9 Hz, $\left.6 \mathrm{H} ;\left(\mathrm{OCH}_{3}\right)_{2}\right)$.

\section{4,5-Dimethoxy-1-[(triisopropylsilyl)ethynyl]-1,2-benziodoxol-3(1H)-one (1d)}

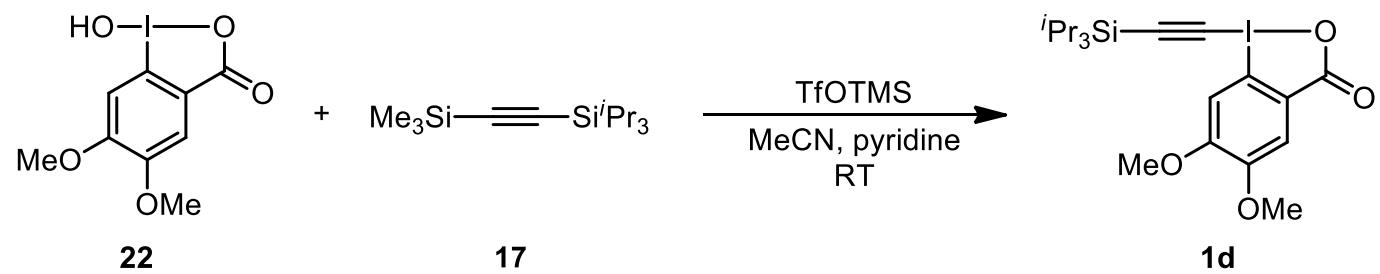

Trimethylsilyltriflate (400 $\mu \mathrm{L}, 2.20 \mathrm{mmol}, 1.10$ equiv, freshly distilled) was added dropwise to a stirred solution of (22) $(648 \mathrm{mg}, 2.00 \mathrm{mmol}, 1.00$ equiv) in acetonitrile $(10 \mathrm{~mL})$. After $2 \mathrm{~min}$, (trimethylsilyl)(triiso-propylsilyl)acetylene (17) $(560 \mathrm{mg}, 2.20 \mathrm{mmol}, 1.10$ equiv) was added dropwise, followed, after $20 \mathrm{~min}$, by the addition of pyridine $(180 \mu \mathrm{L}, 2.20 \mathrm{mmol}$, 1.10 equiv). The mixture was stirred $20 \mathrm{~min}$. The solvent was then removed under reduced pressure and the yellow crude oil was dissolved in dichloromethane $(20 \mathrm{~mL})$. The organic layer was washed with $1 \mathrm{M} \mathrm{HCl}(20 \mathrm{~mL})$ and the aqueous layer was extracted with $\mathrm{CH}_{2} \mathrm{Cl}_{2}(20$ $\mathrm{mL})$. The organic layers were combined, washed with a saturated solution of $\mathrm{NaHCO}_{3}(40$ $\mathrm{mL}$ ), dried over $\mathrm{MgSO}_{4}$, filtered and the solvent was evaporated under reduced pressure. Recrystallization from acetonitrile $(\mathrm{ca} 8 \mathrm{~mL})$ and wash with hexanes afforded $1 \mathbf{1 d}(575 \mathrm{mg}$, $1.18 \mathrm{mmol}, 59 \%$ ) as colorless crystals.

Mp (Dec.) $180-183{ }^{\circ} \mathrm{C} .{ }^{1} \mathrm{H}$ NMR $\left(400 \mathrm{MHz}, \mathrm{CDCl}_{3}\right)(\mathrm{ca} 0.09 \mathrm{mmol} / \mathrm{ml}) \delta 7.83(\mathrm{~s}, 1 \mathrm{H}$; $\operatorname{ArH}), 7.61(\mathrm{~s}, 1 \mathrm{H} ; \operatorname{ArH}), 3.99\left(\mathrm{~s}, 3 \mathrm{H} ; \mathrm{OCH}_{3}\right), 3.97$ (s, $\left.3 \mathrm{H} ; \mathrm{OCH}_{3}\right), 1.14(\mathrm{~m}, 21 \mathrm{H}$; $\left.\mathrm{Si}\left(\mathrm{CH}\left(\mathrm{CH}_{3}\right)_{2}\right)_{3}\right) .{ }^{13} \mathrm{C} \mathrm{NMR}\left(101 \mathrm{MHz}, \mathrm{CDCl}_{3}\right) \delta 166.7,154.9,152.2,124.5,113.8,113.2$, 107.8, 104.7, 66.0, 56.7, 56.5, 18.5, 11.2. IR v $2945(\mathrm{w}), 1616(\mathrm{~m}), 1569(\mathrm{w}), 1497(\mathrm{~m}), 1464$ (w), $1396(\mathrm{~m}), 1317(\mathrm{w}), 1269(\mathrm{~m}), 1215(\mathrm{~m}), 1181(\mathrm{w}), 1129(\mathrm{w}), 1026(\mathrm{w}), 921(\mathrm{w}), 884$ (w), $778(\mathrm{w}), 734(\mathrm{~m}), 708(\mathrm{~m}), 639$ (s). HRMS(ESI) calcd for $\mathrm{C}_{20} \mathrm{H}_{30} \mathrm{O}_{4} \mathrm{ISi}^{+}(\mathrm{M}+\mathrm{H})$ 489.0958, found 489.0950.

\section{3-Methyl-2-iodosylbenzoic acid (24)}

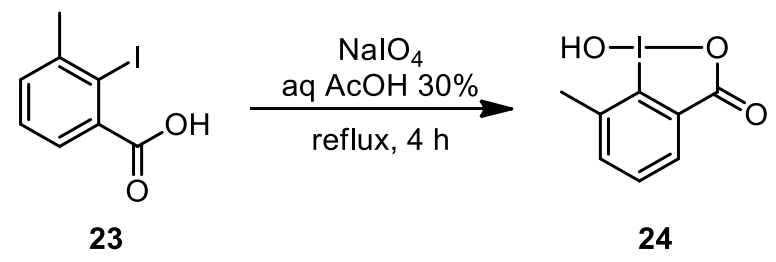

Following the reported procedure, ${ }^{[1]} \mathrm{NaIO}_{4}(1.25 \mathrm{~g}, 5.84 \mathrm{mmol}, 1.05$ equiv) and 2-iodo-3methylbenzoic acid (23) (1.46 g, $5.56 \mathrm{mmol}, 1.00$ equiv) were suspended in $30 \%$ (v:v) aq. $\mathrm{AcOH}(15 \mathrm{~mL})$. The mixture was vigorously stirred and refluxed for $4 \mathrm{~h}$. The reaction mixture 
was then diluted with cold water $(40 \mathrm{~mL})$ and allowed to cool to RT, protecting it from light. The crude product was collected by filtration, washed on the filter with ice water $(3 \times 4 \mathrm{~mL})$ and acetone $(3 \mathrm{x} 4 \mathrm{~mL})$, and air-dried in the dark to give the pure product $24(1.24 \mathrm{~g}, 4.46$ mmol, $80 \%$ ) as a colorless solid.

${ }^{1} \mathrm{H}$ NMR (400 MHz, DMSO) $\delta 8.30$ (br s, $\left.1 \mathrm{H} ; \mathrm{OH}\right), 7.85$ (m, $\left.1 \mathrm{H} ; \mathrm{ArH}\right), 7.57$ (m, $2 \mathrm{H}$; $\operatorname{ArH}), 2.64$ (s, $3 \mathrm{H} ; \operatorname{Ar} H)$.

\section{3-Methyl-1-[(triisopropylsilyl)ethynyl]-1,2-benziodoxol-3(1H)-one (1e)}<smiles>Cc1cccc2c1I(O)OC2=O</smiles>

24<smiles>C[SiH2]C#C[AsH2-][AsH2]</smiles>

17

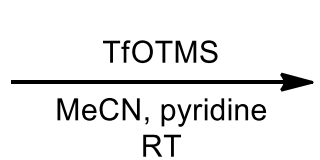

RT<smiles>Cc1cccc2c1I(C#C[SiH3])OC2=O</smiles>

1e

Following a slight modification of the reported procedure, ${ }^{[2]}$ trimethylsilyltriflate $(400 \mu \mathrm{L}$, $2.20 \mathrm{mmol}, 1.1$ equiv, freshly distilled over $\mathrm{CaH}_{2}$ ) was added dropwise to a stirred solution of 24 (556 mg, $2.00 \mathrm{mmol}, 1.0$ equiv) in acetonitrile $(10 \mathrm{~mL})$. After $20 \mathrm{~min}$, (trimethylsilyl)(triiso-propylsilyl)acetylene (17) $(560 \mathrm{mg}, 2.20 \mathrm{mmol}, 1.1$ equiv) was then added dropwise, followed, after $20 \mathrm{~min}$, by the addition of pyridine $(180 \mu \mathrm{L}, 2.20 \mathrm{mmol}, 1.1$ equiv). The mixture was stirred $20 \mathrm{~min}$. The solvent was then removed under reduced pressure and the yellow crude oil was dissolved in dichloromethane $(20 \mathrm{~mL})$. The organic layer was washed with $1 \mathrm{M} \mathrm{HCl}(20 \mathrm{~mL})$ and the aqueous layer was extracted with $\mathrm{CH}_{2} \mathrm{Cl}_{2}(20$ $\mathrm{mL})$. The organic layers were combined, washed with a saturated solution of $\mathrm{NaHCO}_{3}(40$ $\mathrm{mL}$ ), dried over $\mathrm{MgSO}_{4}$, filtered and the solvent was evaporated under reduced pressure. Recrystallization from acetonitrile $(\mathrm{ca} 5 \mathrm{~mL})$ and wash with pentane afforded 1e $(227 \mathrm{mg}$, $0.513 \mathrm{mmol}, 26 \%$ ) as colorless crystals.

Mp (Dec.) $138-145^{\circ} \mathrm{C} .{ }^{1} \mathrm{H} \mathrm{NMR}\left(400 \mathrm{MHz}, \mathrm{CDCl}_{3}\right)(\mathrm{ca} 0.04 \mathrm{mmol} / \mathrm{ml}) \delta 8.21(\mathrm{dd}, J=$ 6.8, $2.5 \mathrm{~Hz}, 1 \mathrm{H} ; \operatorname{ArH}), 7.50(\mathrm{~m}, 2 \mathrm{H} ; \operatorname{ArH}), 2.87\left(\mathrm{~s}, 3 \mathrm{H} ; \mathrm{CH}_{3}\right), 1.10(\mathrm{~m}, 21 \mathrm{H}$; $\left.\mathrm{Si}\left(\mathrm{CH}\left(\mathrm{CH}_{3}\right)_{2}\right)_{3}\right) .{ }^{13} \mathrm{C}$ NMR $\left(101 \mathrm{MHz}, \mathrm{CDCl}_{3}\right) \delta 166.8,140.3,138.0,133.3,131.7,130.8$, 119.1, 112.5, 66.9, 24.0, 18.5, 11.2. IR v $2946(\mathrm{w}), 2867(\mathrm{w}), 2244(\mathrm{w}), 1649(\mathrm{~m}), 1562(\mathrm{w})$, $1464(\mathrm{w}), 1326$ (w), 1281 (w), 998 (w), 907 (s), 884 (w), 763 (w), 728 (s), 687 (s), 647 (m). HRMS(ESI) calcd for $\mathrm{C}_{19} \mathrm{H}_{28} \mathrm{O}_{2} \mathrm{ISi}^{+}(\mathrm{M}+\mathrm{H})$ 443.0903, found 443.0893.

\section{6-Methyl-2-iodosylbenzoic acid (26)}

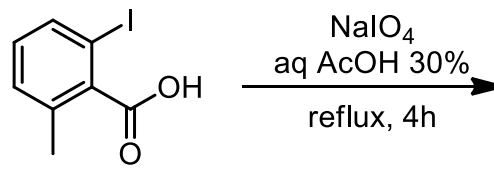

25<smiles>Cc1cccc2c1C(=O)OI2O</smiles>

26

Following the reported procedure, ${ }^{[1]} \mathrm{NaIO}_{4}(2.575 \mathrm{~g}, 12.0 \mathrm{mmol}, 1.05$ equiv) and 2-iodo6-methylbenzoic acid (25) (3.00 g, 11.5 mmol, 1.00 equiv) were suspended in 30\% (v:v) aq. $\mathrm{AcOH}(105 \mathrm{~mL})$. The mixture was vigorously stirred and refluxed for $4 \mathrm{~h}$. The reaction mixture was then diluted with cold water $(75 \mathrm{~mL})$ and allowed to cool to RT, protecting it from light. The crude product was collected by filtration, washed on the filter with ice water $(3 \times 5 \mathrm{~mL})$ and acetone $(3 \times 5 \mathrm{~mL})$, and air-dried in the dark to give the pure product $26(1.24$ g, $4.46 \mathrm{mmol}, 80 \%$ ) as a colorless solid. 
${ }^{1} \mathrm{H}$ NMR (400 MHz, MeOD) $\delta 7.84(\mathrm{~m}, 1 \mathrm{H} ; \operatorname{Ar} H), 7.76(\mathrm{~m}, 1 \mathrm{H} ; \mathrm{ArH}), 7.54(\mathrm{~m}, 1 \mathrm{H}$; $\mathrm{ArH}), 2.78\left(\mathrm{~s}, 3 \mathrm{H} ; \mathrm{CH}_{3}\right)$.

6-Methyl-1-[(triisopropylsilyl)ethynyl]-1,2-benziodoxol-3(1H)-one (1f)<smiles>Cc1cccc2c1C(=O)OI2O</smiles>

26

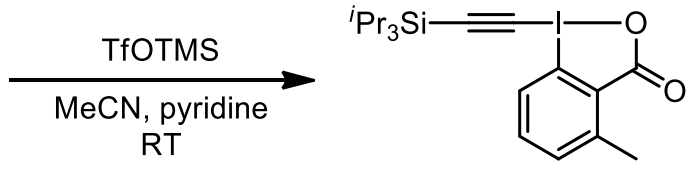

$1 f$

Trimethylsilyltriflate $(1.50 \mathrm{~mL}, 8.27 \mathrm{mmol}, 1.1$ equiv, freshly distilled) was added dropwise to a stirred solution of 26 (2.09 g, $7.52 \mathrm{mmol}, 1.0$ equiv) in acetonitrile $(30 \mathrm{~mL})$. After $20 \mathrm{~min}$, (trimethylsilyl)(triiso-propylsilyl)acetylene (17) (2.10 g, $8.27 \mathrm{mmol}, 1.1$ equiv) was then added dropwise, followed, after $20 \mathrm{~min}$, by the addition of pyridine $(667 \mu \mathrm{L}, 8.27$ mmol, 1.1 equiv). The mixture was stirred $20 \mathrm{~min}$. The solvent was then removed under reduced pressure and the yellow crude oil was dissolved in dichloromethane $(150 \mathrm{~mL})$. The organic layer was washed with $1 \mathrm{M} \mathrm{HCl}(150 \mathrm{~mL})$ and the aqueous layer was extracted with $\mathrm{CH}_{2} \mathrm{Cl}_{2}(150 \mathrm{~mL})$. The organic layers were combined, washed with a saturated solution of $\mathrm{NaHCO}_{3}(150 \mathrm{~mL})$, dried over $\mathrm{MgSO}_{4}$, filtered and the solvent was evaporated under reduced pressure. Recrystallization from acetonitrile and wash with cold acetonitrile afforded $\mathbf{1 f}$ (2.08 $\mathrm{g}, 4.70 \mathrm{mmol}, 63 \%$ ) as colorless crystals.

Mp: $123-125^{\circ} \mathrm{C} .{ }^{1} \mathrm{H}$ NMR $\left(400 \mathrm{MHz}, \mathrm{CDCl}_{3}\right) \delta 8.25(\mathrm{~m}, 1 \mathrm{H} ; \mathrm{ArH}), 7.53(\mathrm{~d}, J=5.2 \mathrm{~Hz}, 2$ $\mathrm{H} ; \mathrm{ArH}), 2.90\left(\mathrm{~s}, 3 \mathrm{H} ; \mathrm{CH}_{3}\right), 1.15\left(\mathrm{~m}, 21 \mathrm{H} ; \mathrm{Si}\left(\mathrm{CH}\left(\mathrm{CH}_{3}\right)_{2}\right)_{3}\right) .{ }^{13} \mathrm{C} \mathrm{NMR}\left(101 \mathrm{MHz}, \mathrm{CDCl}_{3}\right) \delta$ 166.8, 146.7, 135.0, 133.3, 128.7, 124.2, 118.3, 113.3, 68.7, 22.4, 18.5, 11.2. IR $3055(\mathrm{w})$, $2938(\mathrm{~m}), 2873(\mathrm{~m}), 2865(\mathrm{~m}), 2244$ (w), 2089 (w), 1626 (s), 1612 (s), $1586(\mathrm{~m}), 1550(\mathrm{~m})$, $1450(\mathrm{~m}), 1382(\mathrm{w}), 1329(\mathrm{~m}), 1276(\mathrm{w}), 1253$ (w), 1157 (w), $1076(\mathrm{w}), 1018(\mathrm{w}), 998(\mathrm{w})$, $911(\mathrm{w}), 884(\mathrm{~m}), 846(\mathrm{~m}), 817(\mathrm{~m}), 770(\mathrm{~m}), 706(\mathrm{~s}), 679(\mathrm{~s}), 649(\mathrm{~m})$. HRMS (ESI) calcd for $\mathrm{C}_{19} \mathrm{H}_{28} \mathrm{IO}_{2} \mathrm{Si}^{+}[\mathrm{M}+\mathrm{H}]^{+}$443.0898; found 443.0896.

\section{1-Hydroxy-3,3-dimethyl-3-(1H)-1,2-benziodoxole (29)}

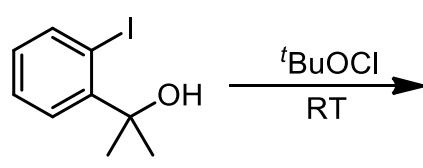

27

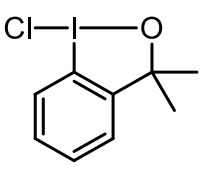

28

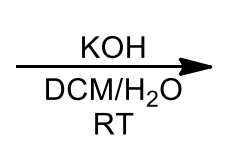

RT

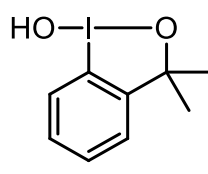

29

Following a reported procedure, ${ }^{[7]} t$ - $\mathrm{BuOCl}(1.0 \mathrm{~mL}, 8.4 \mathrm{mmol}, 1.1$ equiv $)$ was added to 27 (1.95 g, 7.44 mmol, 1.00 equiv) at RT under air in the dark. The reaction was stirred for 30 min at RT. The solvent was removed under vacuum and the resulting solid was dried overnight under high vacuum to afford $28(2.02 \mathrm{~g}, 6.82 \mathrm{mmol}, 92 \%)$ as a solid.

${ }^{1} \mathrm{H}$ NMR (400 MHz, $\left.\mathrm{CDCl}_{3}\right) \delta 8.03(\mathrm{dd}, 1 \mathrm{H}, J=8.1,1.3 \mathrm{~Hz}, \mathrm{ArH}), 7.55(\mathrm{~m}, 2 \mathrm{H}, \mathrm{ArH})$, 7.17 (dd, $1 \mathrm{H}, J=7.3,1.7 \mathrm{~Hz}, \operatorname{ArH}), 1.55$ (s, $6 \mathrm{H}, \mathrm{Me})$.

The solid 28 (2.02 g, $6.82 \mathrm{mmol}, 1.00$ equiv) was then dissolved in DCM (30 mL) and $\mathrm{KOH}$ (382 mg, $6.82 \mathrm{mmol}, 1.00$ equiv) in water $(3 \mathrm{~mL})$ was added. After $3 \mathrm{~h}$, the organic layer was separated, dried over $\mathrm{MgSO}_{4}$, filtered over $\mathrm{MgSO}_{4}$ and concentrated. The resulting

[7] Moss, R. A.; Wilk, B.; Kroghjespersen, K.; Blair, J. T.; Westbrook, J. D. J. Am. Chem. Soc. 1989, $111,250$. 
solid was recrystallized in EtOAc, washed with hexanes and dried under vacuum to afford 29 $(1.11 \mathrm{~g}, 3.99 \mathrm{mmol}, 59 \%)$ as a colorless solid.

Mp (Dec.) $241-243{ }^{\circ} \mathrm{C} .{ }^{1} \mathrm{H}$ NMR $(400 \mathrm{MHz}, \mathrm{DMSO}) \delta 7.79(\mathrm{~d}, J=7.9 \mathrm{~Hz}, 1 \mathrm{H}$; ArH), $7.52(\mathrm{~m}, 2 \mathrm{H}$; $\mathrm{ArH}), 7.36(\mathrm{~d}, J=7.4 \mathrm{~Hz}, 1 \mathrm{H} ; \mathrm{ArH}), 1.36\left(\mathrm{~s}, 6 \mathrm{H} ;\left(\mathrm{CH}_{3}\right)_{2}\right) .{ }^{13} \mathrm{C}$ NMR $(101$ $\left.\mathrm{MHz}, \mathrm{CDCl}_{3}\right) \delta 150.0,129.7,129.2,127.0,126.2,115.3,79.4,30.2 . \mathrm{IR} v 1569(\mathrm{w}), 1464(\mathrm{w})$, 1443 (w), 1263 (s), 1185 (s), 1140 (s), 1110 (s), 1020 (w), 952 (s), 761 (m), 730 (m), 690 (m), $643(\mathrm{~s})$.

\section{1-[(Triiso-propylsilyl)ethynyl]-1,3-dihydro-3,3-dimethyl-1,2-benziodoxolone (5)}<smiles>CC1(C)OI(O)c2ccccc21</smiles>

29

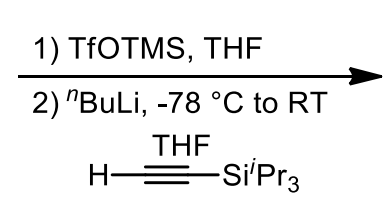

$\mathrm{H} \stackrel{\text { THF }}{=} \mathrm{Si}^{i} \mathrm{Pr}_{3}$

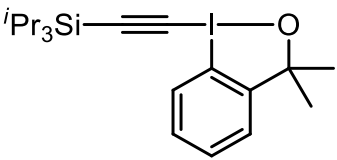

5

Trimethylsilyltriflate $(250 \mu \mathrm{L}, 1.38 \mathrm{mmol}, 1.00$ equiv) was added to a stirring solution of 29 (408 mg, $1.38 \mathrm{mmol}, 1.00$ equiv) in THF (40 mL) at RT. The solution was stirred at RT for $20 \mathrm{~min}$ and then cooled to $-78{ }^{\circ} \mathrm{C}$. In the meantime, $n$-BuLi $(2.5 \mathrm{M}$ in hexanes, $550 \mu \mathrm{L}, 1.38$ mmol, 1.00 equiv) was added to a stirring solution of triisopropylacetylene $(310 \mu \mathrm{L}, 1.38$ mmol, 1.00 equiv) in THF $(10 \mathrm{~mL})$ at $-78{ }^{\circ} \mathrm{C}$. The solution was stirred for $30 \mathrm{~min}$ at $-78{ }^{\circ} \mathrm{C}$ and then added via cannula to the first solution. The reaction was stirred for $1 \mathrm{~h}$ at $-78{ }^{\circ} \mathrm{C}$, warmed to RT and stirred $4 \mathrm{~h}$. The reaction was quenched with saturated $\mathrm{NH}_{4} \mathrm{Cl}(20 \mathrm{~mL})$. The layers were separated and the aqueous layers were extracted with DCM $(20 \mathrm{~mL})$. The organic layers were combined, washed with brine, dried over $\mathrm{MgSO}_{4}$, filtered and reduced under vacuum. The resulting oil was then purified by column chromatography ( $\mathrm{PET} / \mathrm{Et}_{2} \mathrm{O}$ 6/4) to afford 5 (524 mg, $1.18 \mathrm{mmol}, 86 \%)$ as a yellow oil which crystallize at $-18{ }^{\circ} \mathrm{C}$.

$\mathrm{R}_{f} 0.15\left(\mathrm{PET}_{\mathrm{Et}} \mathrm{O}\right.$ 6:4, UV). Mp 59-61 ${ }^{\circ} \mathrm{C} .{ }^{1} \mathrm{H}$ NMR $\left(400 \mathrm{MHz}, \mathrm{CDCl}_{3}\right)$ (ca 0.16 $\mathrm{mmol} / \mathrm{ml}) \delta 8.23(\mathrm{dd}, 1 \mathrm{H}, J=8.2,0.9 \mathrm{~Hz}, \mathrm{ArH}), 7.52(\mathrm{td}, 1 \mathrm{H}, J=7.3,1.0 \mathrm{~Hz}, \operatorname{ArH}), 7.41$ (ddd, $1 \mathrm{H}, J=8.6,7.2,1.5 \mathrm{~Hz}, \mathrm{ArH}), 7.35(\mathrm{dd}, 1 \mathrm{H}, J=7.5,1.5 \mathrm{~Hz}, \mathrm{ArH}), 1.44$ (s, $6 \mathrm{H}, \mathrm{Me}$ ), 1.12 (s, $21 \mathrm{H}, \mathrm{TIPS}) .{ }^{13} \mathrm{C} \mathrm{NMR}\left(101 \mathrm{MHz}, \mathrm{CDCl}_{3}\right) \delta 148.0,130.4,129.2,127.4,126.5,111.0$, 105.8, 80.8, 75.7, 31.5, 18.6, 11.4. IR $v 2945$ (m), $2864(\mathrm{~m}), 2064(\mathrm{w}), 1690(\mathrm{w}), 1562(\mathrm{w})$, 1462 (m), 1436 (m), 1355 (w), 1244 (w), 1162 (w), $1116(\mathrm{w}), 1073$ (w), 999 (m), 968 (m), $883(\mathrm{~m}), 756$ (m), 691 (s). HRMS(ESI) calcd for $\mathrm{C}_{20} \mathrm{H}_{32} \mathrm{OISi}^{+}(\mathrm{M}+\mathrm{H})$ 443.1267, found 443.1276 .

\section{1-Chloro-1,3-dihydro-3,3-bis(trifluoromethyl)-1,2-benziodoxole (32)}<smiles>OC(c1ccccc1)(C(F)(F)F)C(F)(F)F</smiles>

30

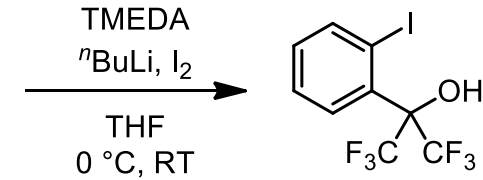

31

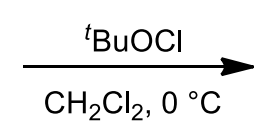<smiles>FC(F)(F)C(F)(F)C(F)(F)C(F)(F)C(F)(F)F</smiles>

32

Following a reported procedure, ${ }^{[8]}$ TMEDA (distilled over $\left.\mathrm{KOH}\right)(0.630 \mathrm{~mL}, 4.15 \mathrm{mmol}$, 0.200 equiv) was added to a solution of $n-\operatorname{BuLi}(2.5 \mathrm{M}$ in hexanes, $18.3 \mathrm{~mL}, 45.8 \mathrm{mmol}, 2.20$

[8] E. F. Perozzi, R. S. Michalak, G. D. Figuly, W. H. Stevenson, D. B. Dess, M. R. Ross, J. C. Martin, J. Org. Chem. 1981, 46, 1049; 
equiv). After $15 \mathrm{~min}$, the cloudy solution was cooled to $0{ }^{\circ} \mathrm{C}$ and $\mathbf{3 0}(3.50 \mathrm{~mL}, 20.8 \mathrm{mmol}$, 1.00 equiv) in THF ( $3 \mathrm{~mL})$ was added dropwise. The reaction was stirred $30 \mathrm{~min}$ at $0{ }^{\circ} \mathrm{C}$ and then $18 \mathrm{~h}$ at RT. $\mathrm{I}_{2}\left(5.60 \mathrm{~g}, 22.1 \mathrm{mmol}, 1.06\right.$ equiv) was then added portionwise at $0{ }^{\circ} \mathrm{C}$ and the mixture was stirred at $0{ }^{\circ} \mathrm{C}$ for $30 \mathrm{~min}$ and then $4 \mathrm{~h}$ at RT. The reaction was quenched with saturated $\mathrm{NH}_{4} \mathrm{Cl}$. $\mathrm{Et}_{2} \mathrm{O}(50 \mathrm{~mL})$ was added and the layers were separated. The aqueous layer was then extracted twice with $\mathrm{Et}_{2} \mathrm{O}(2 \times 50 \mathrm{~mL})$. The organic layers were combined, washed twice with saturated $\mathrm{NaS}_{2} \mathrm{O}_{3}(2 \times 50 \mathrm{~mL})$, dried over $\mathrm{MgSO}_{4}$, filtered and reduced to afford $7.83 \mathrm{~g}$ of $\mathbf{3 1}$ as orange oil which was used without further purification.

The crude oil was dissolved in wet dichloromethane $(20 \mathrm{~mL})$ in the dark under air. $t$ $\mathrm{BuOCl}\left(2.60 \mathrm{~mL}, 21.8 \mathrm{mmol}, 1.05\right.$ equiv) was then added dropwise at $0{ }^{\circ} \mathrm{C}$. After $30 \mathrm{~min}$, the resulting suspension was filtered to afford $32(3.52 \mathrm{~g}, 8.70 \mathrm{mmol}, 42 \%)$ as a yellow oil. The mother liquors were carefully reduced to one third and filtered to afford $(2.33 \mathrm{~g}, 5.76 \mathrm{mmol}$, $28 \%$ ) as a yellow solid. Combined yield: $70 \%$.

Mp (Dec.) $167-169^{\circ} \mathrm{C} .{ }^{1} \mathrm{H}$ NMR $\left(400 \mathrm{MHz}, \mathrm{CDCl}_{3}\right) \delta 8.09(\mathrm{~d}, J=8.4 \mathrm{~Hz}, 1 \mathrm{H} ; \mathrm{ArH}), 7.85$ $(\mathrm{m}, 1 \mathrm{H} ; \mathrm{Ar} H), 7.73(\mathrm{~m}, 2 \mathrm{H}$; $\mathrm{ArH}) .{ }^{13} \mathrm{C} \mathrm{NMR}\left(101 \mathrm{MHz}, \mathrm{CDCl}_{3}\right) \delta 133.8,132.1,131.6$, 129.7, 128.5, 122.8 (q, $289 \mathrm{~Hz}$ ). 113.4, 84.8. The characterization data corresponded to the reported values. ${ }^{[9]}$

\section{1-Hydroxy-3,3-bis(trifluoromethyl)-3-(1H)-1,2-benziodoxole (33)}

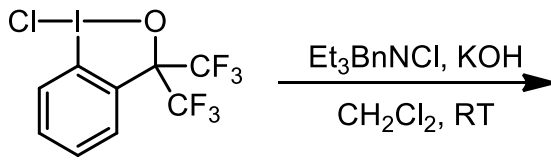

32<smiles>OI1OC(C(F)(F)F)(C(F)(F)F)c2ccccc21</smiles>

33

Following a reported procedure, ${ }^{[10]} \mathrm{Et}_{3} \mathrm{BnNCl}(83 \mathrm{mg}, 0.36 \mathrm{mmol}, 0.050$ equiv) was added to a stirring solution of $\mathbf{3 2}(3.00 \mathrm{~g}, 7.41 \mathrm{mmol}, 1.00$ equiv) in dichloromethane $(50 \mathrm{~mL})$ and $\mathrm{KOH}(415 \mathrm{mg}, 7.41 \mathrm{mmol}, 1.00$ equiv) in water $(8 \mathrm{~mL})$. The reaction was stirred for $3 \mathrm{~h} 30$ min under air. The organic layer was separated and dried over $\mathrm{MgSO}_{4}$. The resulting solid was purified over a silica plug with EtOAc and then recrystallized in EtOAc, washed in pentane to afford $33(1.24 \mathrm{~g}, 3.21 \mathrm{mmol}, 43 \%)$ as a colorless solid. The mother liquors were reduced and recrystallized in EtOAc to afford a second batch of $\mathbf{3 3}$ (279 $\mathrm{mg}, 0.723 \mathrm{mmol}, 10$ $\%)$ as a colorless solid. Combined yield: $53 \%$.

${ }^{1} \mathrm{H}$ NMR (400 MHz, DMSO) $\delta 7.96$ (m, $2 \mathrm{H}$; ArH), 7.73 (m, $2 \mathrm{H}$; ArH). ${ }^{13} \mathrm{C}$ NMR (101 MHz, DMSO) $\delta 133.3,131.0,130.8,128.9,127.9,123.4$ (q, $J=290 \mathrm{~Hz}), 117.2,83.7(\mathrm{~m})$. IR v $1464(\mathrm{w}), 1435$ (w), 1290 (w), 1263 (m), 1185 (s), 1139 (s), 1103 (m), $1041(\mathrm{w}), 1021(\mathrm{w})$, $952(\mathrm{~s}), 760(\mathrm{~m}), 730(\mathrm{~m}), 692(\mathrm{~m})$.

\section{1-[(Triisopropylsilyl)ethynyl]-3,3-bis(trifluoromethyl)-3(1H)-1,2-benziodoxole (6)}

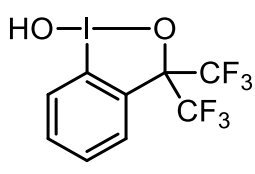

33

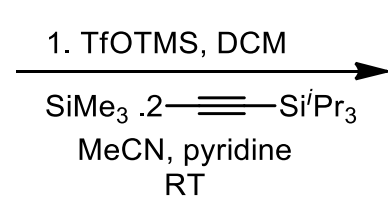

RT

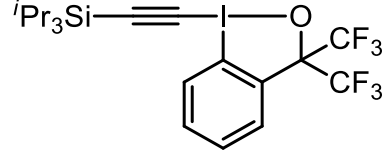

6

[9] J. Cvengros, D. Stolz, A. Togni, Synthesis 2009, 2818.

[10]A. J. Blake, M. Novak, R. I. Davies, S. Robinson, S. Woodward, Synth. Commun. 2009, 39, 1065. 
Trimethylsilyltriflate $(310 \mu \mathrm{L}, 1.71 \mathrm{mmol}, 1.10$ equiv, freshly distilled, $)$ was added to a stirred solution of 33 (600 mg, $1.55 \mathrm{mmol}, 1.00$ equiv) in dichloromethane (20 mL) at RT. After $20 \mathrm{~min}$, the solution was reduced and the resulting oil was dissolved in $\mathrm{CH}_{3} \mathrm{CN}$ (30 $\mathrm{mL}$ ). (Trimethylsilyl)(triiso-propylsilyl)acetylene (514 mg, $1.71 \mathrm{mmol}, 1.10$ equiv) was added and after $20 \mathrm{~min}$ pyridine ( $76 \mu \mathrm{L}, 0.94 \mathrm{mmol}, 0.60$ equiv) was added. The reaction was then reduced under vacuum, dissolved in $\mathrm{Et}_{2} \mathrm{O}$ and filtered over a silica plug (eluent $\mathrm{Et}_{2} \mathrm{O}$ ). The resulting solid was purified by column chromatography (PET/Et $2 \mathrm{O} 95 / 5)$ to afford $6(816 \mathrm{mg}$, $1.48 \mathrm{mmol}, 95 \%$ ) as a colorless solid.

$\mathrm{R}_{f}\left(\mathrm{PET} / \mathrm{Et}_{2} \mathrm{O}\right.$ 95:5, UV): 0.4. Mp (Dec.) 131-132 ${ }^{\circ} \mathrm{C} .{ }^{1} \mathrm{H} \mathrm{NMR}\left(400 \mathrm{MHz}, \mathrm{CDCl}_{3}\right)(\mathrm{ca}$ $0.10 \mathrm{mmol} / \mathrm{ml}) \delta 8.36(\mathrm{dd}, J=7.9,1.7 \mathrm{~Hz}, 1 \mathrm{H} ; \mathrm{ArH}), 7.84(\mathrm{~d}, J=6.7 \mathrm{~Hz}, 1 \mathrm{H} ; \mathrm{ArH}), 7.68$ $(\mathrm{m}, 2 \mathrm{H} ; \mathrm{ArH}), 1.15\left(\mathrm{~m}, 21 \mathrm{H} ; \mathrm{Si}\left(\mathrm{CH}\left(\mathrm{CH}_{3}\right)_{2}\right)_{3}\right) .{ }^{13} \mathrm{C} \mathrm{NMR}\left(101 \mathrm{MHz}, \mathrm{CDCl}_{3}\right) \delta 132.7,131.1$, 129.9, 129.9 (m) 128.2, 123.6 (q, $288 \mathrm{~Hz})$ 112.1, 110.8, 81.4 (m), 69.7, 18.5, 11.2. IR v 2947 (m), $2868(\mathrm{~m}), 2249(\mathrm{w}), 1566(\mathrm{w}), 1465(\mathrm{~m}), 1438(\mathrm{w}), 1387(\mathrm{w}), 1264(\mathrm{~s}), 1218(\mathrm{~m}), 1184$ (s), 1149 (s), 1071 (w), 994 (w), 951 (s), 910 (m), 873 (w), 732 (s), 696 (s), 655 (s), 655 (s). HRMS(ESI) calcd for $\mathrm{C}_{20} \mathrm{H}_{26} \mathrm{OF}_{6} \mathrm{ISi}^{+}(\mathrm{M}+\mathrm{H})$ 551.0702, found 551.0723.

\section{1-[Phenylethynyl]-1,2-benziodoxol-3(1H)-one (8a)}

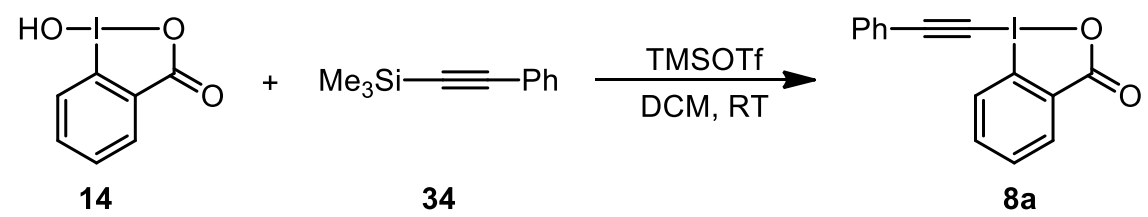

Trimethylsilyl triflate $(7.50 \mathrm{~mL}, 41.5 \mathrm{mmol}, 1.10$ equiv) was added to a suspension of 2iodosylbenzoic acid (14) (10.0 g, $37.7 \mathrm{mmol}, 1.00$ equiv) in $\mathrm{CH}_{2} \mathrm{Cl}_{2}(100 \mathrm{~mL})$ at RT. The resulting yellow mixture was stirred for $1 \mathrm{~h}$, followed by the dropwise addition of trimethyl(phenylethynyl)silane (34) $(8.10 \mathrm{~mL}, 41.5 \mathrm{mmol}, 1.10$ equiv) (slightly exothermic). The resulting suspension was stirred for $6 \mathrm{~h}$ at RT, during this time a white solid was formed. A saturated solution of $\mathrm{NaHCO}_{3}(100 \mathrm{~mL})$ was then added and the mixture was stirred vigorously. The resulting suspension was filtered on porosity 4 . The two layers of the mother liquors were separated and the organic layer was washed with sat. $\mathrm{NaHCO}_{3}$, dried over $\mathrm{MgSO}_{4}$, filtered and evaporated under reduced pressure. The resulting mixture was combined with the solid obtained by filtration and boiled in $\mathrm{CH}_{3} \mathrm{CN}(300 \mathrm{~mL})$. The mixture was cooled down, filtered and dried under high vacuum to afford 8a (6.08 $\mathrm{g}, 17.4 \mathrm{mmol}, 46 \%)$ as a colorless solid.

${ }^{1} \mathrm{H}$ NMR $\left(400 \mathrm{MHz}, \mathrm{CDCl}_{3}\right)(\mathrm{ca} 0.03 \mathrm{mmol} / \mathrm{ml}) \delta 8.46(\mathrm{~m}, 1 \mathrm{H} ; \mathrm{ArH}), 8.28(\mathrm{~m}, 1 \mathrm{H}$; $\operatorname{Ar} H), 7.80(\mathrm{~m}, 3 \mathrm{H} ; \operatorname{Ar} H), 7.63(\mathrm{~m}, 2 \mathrm{H}$; $\operatorname{Ar} H), 7.48(\mathrm{~m}, 3 \mathrm{H} ; \operatorname{Ar} H) .{ }^{13} \mathrm{C}$ NMR $(101 \mathrm{MHz}$, $\left.\mathrm{CDCl}_{3}\right) \delta 163.9,134.9,132.9,132.5,131.6,130.8,128.8,126.2,120.5,116.2,106.6,50.2$. The characterization data corresponded to the reported values. ${ }^{[2]}$

5-Nitro-1-[phenylethynyl]-1,2-benziodoxol-3(1H)-one $(8 \mathrm{~b})$<smiles>O=C1OI(O)c2ccc([N+](=O)[O-])cc21</smiles>

19<smiles>C[SiH2]C#Cc1ccccc1</smiles>

34

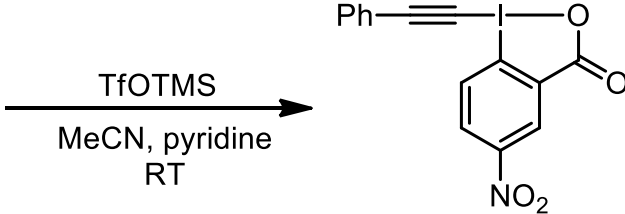

$8 b$ 
Trimethylsilyltriflate ( $646 \mu \mathrm{L}, 3.56 \mathrm{mmol}, 1.10$ equiv, freshly distilled over $\mathrm{CaH}_{2}$ ) was added dropwise to a stirred solution of 2-iodosylbenzoic acid 19 (1.00 g, $3.23 \mathrm{mmol}, 1.00$ equiv) in DCM (10 mL). After $1 \mathrm{~h}$ at RT, trimethyl(phenylethynyl)silane (34) $(696 \mu \mathrm{L}, 3.56$ mmol, 1.10 equiv) was then added dropwise. The mixture was stirred $6 \mathrm{hrs}$. A saturated solution of $\mathrm{NaHCO}_{3}(10 \mathrm{~mL})$ was added. The resulting suspension was filtered and washed with acetone $(2 \mathrm{~mL})$. The resulting solid was dried overnight under high vacuum to afford $\mathbf{8 b}$ (778 $\mathrm{mg}, 1.98 \mathrm{mmol}, 62 \%$ ) as a colorless solid.

$\mathrm{Mp}$ (Dec.) $198-202{ }^{\circ} \mathrm{C} .{ }^{1} \mathrm{H}$ NMR $\left(400 \mathrm{MHz}, \mathrm{CDCl}_{3}\right)(\mathrm{ca} 0.01 \mathrm{mmol} / \mathrm{ml}) \delta 9.18(\mathrm{~d}, J=2.6$ $\mathrm{Hz}, 1 \mathrm{H}$; ArH), 8.59 (dd, $J=8.9,2.6 \mathrm{~Hz}, 1 \mathrm{H} ; \operatorname{Ar} H), 8.48(\mathrm{~d}, J=8.9 \mathrm{~Hz}, 1 \mathrm{H} ; \operatorname{Ar} H), 7.62(\mathrm{~m}$, $2 \mathrm{H} ; \mathrm{ArH}), 7.54(\mathrm{~m}, 1 \mathrm{H} ; \mathrm{ArH}), 7.47(\mathrm{~m}, 2 \mathrm{H} ; \mathrm{ArH}){ }^{13} \mathrm{C} \mathrm{NMR}\left(101 \mathrm{MHz}, \mathrm{CDCl}_{3} / \mathrm{DMSO}_{7 / 2}\right) \delta$ 164.0, 149.5, 133.4, 131.7, 129.8, 128.2, 127.7, 127.4, 124.8, 121.7, 119.2, 105.3, 49.5. IR v $3082(\mathrm{w}), 2155$ (w), $1625(\mathrm{~s}), 1569(\mathrm{w}), 1532(\mathrm{w}), 1450(\mathrm{w}), 1342(\mathrm{~m}), 1171(\mathrm{w}), 1074(\mathrm{w})$, $1024(\mathrm{w}), 910(\mathrm{~m}), 825(\mathrm{w}), 792(\mathrm{w}), 734(\mathrm{~s}), 693$ (w), 636 (w). HRMS(ESI) calcd for $\mathrm{C}_{15} \mathrm{H}_{9} \mathrm{NO}_{4} \mathrm{I}^{+}(\mathrm{M}+\mathrm{H})$ 393.9576, found 393.9591.

\section{5-Fluoro-2-iodosylbenzoic acid (36)}<smiles>O=C(O)c1cc(F)ccc1I</smiles>

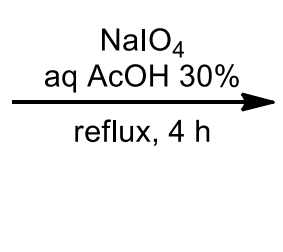<smiles>O=C1OI(O)c2ccc(F)cc21</smiles>

Following the reported procedure, ${ }^{[1]} \mathrm{NaIO}_{4}(656 \mathrm{mg}, 30.7 \mathrm{mmol}, 1.05$ equiv) and 2-iodo-4fluorobenzoic acid (35) (778 g, $29.2 \mathrm{mmol}, 1.00$ equiv) were suspended in 30\% (v:v) aq. $\mathrm{AcOH}(7 \mathrm{~mL})$. The mixture was vigorously stirred and refluxed for $4 \mathrm{~h}$. The reaction mixture was then diluted with cold water $(20 \mathrm{~mL})$ and allowed to cool to RT, protecting it from light. The crude product was collected by filtration, washed on the filter with ice water $(3 \times 4 \mathrm{~mL})$ and acetone $(3 \times 4 \mathrm{~mL})$, and air-dried in the dark to give the pure product 36 (738 $\mathrm{mg}, 2.62$ mmol, $90 \%$ ) as a colorless solid.

${ }^{1} \mathrm{H}$ NMR (400 MHz, DMSO) $\delta$ 7.88-7.79 (m, $\left.3 \mathrm{H} ; \mathrm{Ar} H+\mathrm{OH}\right), 7.75(\mathrm{~m}, 1 \mathrm{H} ; \mathrm{Ar} H)$.

\section{5-Fluoro-1-[phenylethynyl]-1,2-benziodoxol-3(1H)-one $(8 \mathrm{c})$}

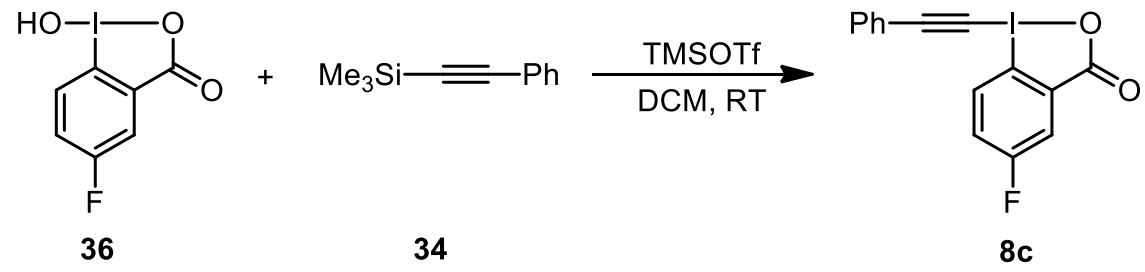

Trimethylsilyl triflate $(231 \mu \mathrm{L}, 1.28 \mathrm{mmol}, 1.10$ equiv) was added to a suspension of 2iodosylbenzoic acid 36 (330 mg, $1.16 \mathrm{mmol}, 1.00$ equiv) in $\mathrm{CH}_{2} \mathrm{Cl}_{2}(5 \mathrm{~mL})$ at $\mathrm{RT}$. The resulting yellow mixture was stirred for $1 \mathrm{~h}$, followed by the dropwise addition of trimethyl(phenylethynyl)silane $(252 \mu \mathrm{L}, 1.28 \mathrm{mmol}, 1.10$ equiv) (slightly exothermic). The resulting suspension was stirred for $6 \mathrm{~h}$ at RT, during this time a white solid was formed. A saturated solution of $\mathrm{NaHCO}_{3}(5 \mathrm{~mL})$ was then added and the mixture was stirred vigorously until completely solubilization of the white solid. The two layers were separated and the combined organic extracts were washed with sat. $\mathrm{NaHCO}_{3}$, dried over $\mathrm{MgSO}_{4}$, filtered and 
evaporated under reduce pressure. Recrystallization from acetonitrile $(20 \mathrm{~mL})$ afforded 8c (176 mg, $0.480 \mathrm{mmol}, 41 \%$ ) as a colorless microcrystalline solid.

Mp (Dec.) $147-152{ }^{\circ} \mathrm{C} .{ }^{1} \mathrm{H}$ NMR (400 MHz, DMSO) $\delta 8.30(\mathrm{dd}, J=8.9,4.5 \mathrm{~Hz}, 1 \mathrm{H}$; $\operatorname{ArH}), 7.85(\mathrm{dd}, J=8.3,2.9 \mathrm{~Hz}, 1 \mathrm{H} ; \mathrm{ArH}), 7.79(\mathrm{~m}, 1 \mathrm{H} ; \mathrm{ArH}), 7.71(\mathrm{~m}, 2 \mathrm{H} ; \mathrm{ArH}), 7.60-7.48$ $\left(\mathrm{m}, 3 \mathrm{H}\right.$; ArH). ${ }^{13} \mathrm{C}$ NMR (101 MHz, DMSO) $\delta 164.9(\mathrm{~s}), 164.3(\mathrm{~d}, J=249 \mathrm{~Hz}), 134.9$ (d, $J=$ $7 \mathrm{~Hz}), 132.6$ (s), 130.7 (s), 129.7 (d, $J=9 \mathrm{~Hz}), 129.0$ (s), 122.3 (d, $J=24 \mathrm{~Hz}), 120.3$ (s), 117.7 (d, $J=24 \mathrm{~Hz}), 109.9$ (s), 104.5 (s), 51.5 (s). IR v 3089 (w), 3037 (w), 2153 (w), 1632 (s), 1615 (s), 1597 (m), 1569 (m), 1494 (w), 1449 (s), 1306 (s), 1247 (m), 1203 (m), 1130 (w), $1085(\mathrm{w}), 1006$ (w), 920 (m), $900(\mathrm{w}), 847$ (m), 790 (s), 784 (s), 753 (s). HRMS(ESI) calcd for $\mathrm{C}_{15} \mathrm{H}_{9} \mathrm{O}_{2} \mathrm{FI}^{+}(\mathrm{M}+\mathrm{H})$ 366.9631, found 366.9626.

\section{4,5-Dimethoxy-1-[phenylethynyl]-1,2-benziodoxol-3(1H)-one $(8 \mathrm{~d})$}<smiles>COc1cc2c(cc1OC)I(O)OC2=O</smiles>

22

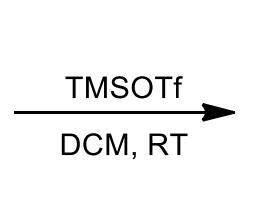

34<smiles>COc1cc2c(cc1OC)I(C#Cc1ccccc1)OC2=O</smiles>

8d

Trimethylsilyl triflate ( $400 \mu \mathrm{L}, 2.20 \mathrm{mmol}, 1.10$ equiv) was added to a suspension of 22 (648 mg, $2.00 \mathrm{mmol}, 1.00$ equiv) in $\mathrm{CH}_{2} \mathrm{Cl}_{2}(10 \mathrm{~mL}$ ) at $\mathrm{RT}$. The resulting yellow mixture was stirred for $1 \mathrm{~h}$, followed by the dropwise addition of trimethyl(phenylethynyl)silane (34) (430 $\mu \mathrm{L}, 2.20 \mathrm{mmol}, 1.10$ equiv). The resulting suspension was stirred for $6 \mathrm{~h}$ at $\mathrm{RT}$, during this time a yellow suspension was formed. A saturated solution of $\mathrm{NaHCO}_{3}(10 \mathrm{~mL})$ was then added. The two layers were separated and the aqueous layer was extracted with DCM (10 $\mathrm{mL}$ ). The combined organic extracts were dried over $\mathrm{MgSO}_{4}$, filtered and evaporated under reduced pressure. The resulting solid was recrystallized in $\mathrm{CH}_{3} \mathrm{CN}(50 \mathrm{~mL})$ and a few EtOH to afford 8d (322 $\mathrm{mg}, 0.789 \mathrm{mmol}, 39 \%)$ as a colorless solid. The mother liquors was reflux in $\mathrm{CH}_{3} \mathrm{CN}(4 \mathrm{~mL})$, filtered and washed with cold $\mathrm{CH}_{3} \mathrm{CN}$ and pentane and dried under high vacuum to afford $\mathbf{8 d}$ (341 $\mathrm{mg}, 0.835 \mathrm{mmol}, 42 \%$ ) as a colorless solid. Combined yield: $81 \%$.

Mp (Dec.) $176-179{ }^{\circ} \mathrm{C} .{ }^{1} \mathrm{H}$ NMR $\left(400 \mathrm{MHz}, \mathrm{CDCl}_{3}\right)(\mathrm{ca} 0.08 \mathrm{mmol} / \mathrm{ml}) \delta 7.89(\mathrm{~s}, 1 \mathrm{H}$; $\operatorname{ArH}), 7.70(\mathrm{~s}, 1 \mathrm{H} ; \mathrm{ArH}), 7.60(\mathrm{~m}, 2 \mathrm{H} ; \mathrm{ArH}), 7.50(\mathrm{~m}, 3 \mathrm{H} ; \mathrm{ArH}), 4.05$ (s, $\left.3 \mathrm{H} ; \mathrm{CH}_{3}\right), 3.98$ (s, $\left.3 \mathrm{H} ; \mathrm{CH}_{3}\right) .{ }^{13} \mathrm{C}$ NMR $\left(101 \mathrm{MHz}, \mathrm{CDCl}_{3}\right) \delta 166.8,154.9,152.2,132.6,130.8,128.9,124.4$, 120.5, 113.3, 107.6, 106.3, 105.3, 56.7, 56.4, 51.2. IR v $3079(\mathrm{w}), 2935(\mathrm{w}), 2854(\mathrm{w}), 2147$ (w), 1742 (w), 1623 (s), 1569 (m), 1497 (s), 1442 (m), 1396 (s), 1300 (m), 1272 (s), 1215 (s), $1167(\mathrm{~m}), 1128(\mathrm{w}), 1023(\mathrm{~m}), 906(\mathrm{w}), 781(\mathrm{~m}), 735(\mathrm{~s}), 656(\mathrm{w}), 635$ (s). HRMS(ESI) calcd for $\mathrm{C}_{17} \mathrm{H}_{14} \mathrm{O}_{4} \mathrm{I}^{+}(\mathrm{M}+\mathrm{H})$ 408.9937, found 408.9949.

(4-Nitrophenyl)ethynyl)trimethylsilane (38)

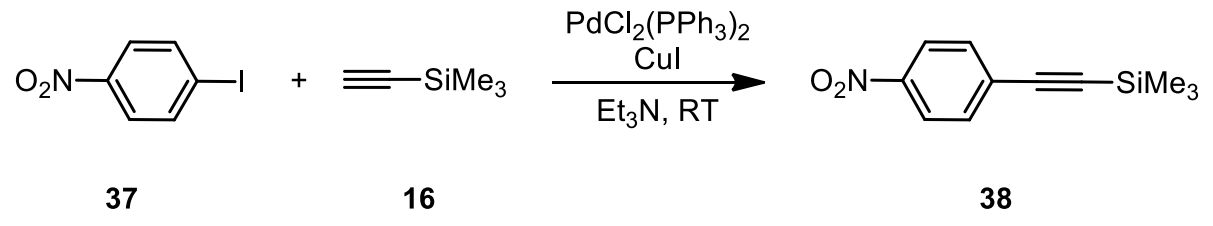


Following a slight modification of the reported procedure, ${ }^{[11]}$ 1-iodo-4-nitrobenzene (37) (1.06 g, $4.27 \mathrm{mmol}, 1.00$ equiv) was dissolved in $\mathrm{Et}_{3} \mathrm{~N}(10 \mathrm{~mL})$ (without prior drying). After three freeze-thaw-pump cycle, $\mathrm{PdCl}_{2}\left(\mathrm{PPh}_{3}\right)_{2}(30 \mathrm{mg}, 0.42 \mathrm{mmol}, 0.10$ equiv) and $\mathrm{CuI}(16 \mathrm{mg}$, $0.84 \mathrm{mmol}, 0.20$ equiv) were added under $\mathrm{N}_{2}$. After the addition of trimethylsilylacetylene (16) $(1.2 \mathrm{~mL}, 8.5 \mathrm{mmol}, 2$ equiv), the green suspension was stirred at RT for $1 \mathrm{~h}$. The reaction mixture was reduced under vacuum, dissolved in DCM $(30 \mathrm{~mL})$, washed with $5 \%$ EDTA solution and water. Organic layers were then dried over $\mathrm{MgSO}_{4}$, filtered and reduced under vacuum. The resulting oil was purified by column chromatography (PET/EtOAc 99/1) to afford 38 (890 mg, $4.06 \mathrm{mmol}, 95 \%)$.

$\mathrm{R}_{f} 0.3$ (PET/EtOAc 99:1, UV). ${ }^{1} \mathrm{H}$ NMR $\left(400 \mathrm{MHz}, \mathrm{CDCl}_{3}\right) \delta 8.17(\mathrm{~d}, J=8.6,2 \mathrm{~Hz}, 2 \mathrm{H}$; $\operatorname{Ar} H), 7.59(\mathrm{~d}, J=8.6,2 \mathrm{H} ; \mathrm{ArH}), 0.27\left(\mathrm{~s}, 9 \mathrm{H} ; \mathrm{Si}\left(\mathrm{CH}_{3}\right)_{3}\right) .{ }^{13} \mathrm{C} \mathrm{NMR}\left(101 \mathrm{MHz}, \mathrm{CDCl}_{3}\right) \delta$ $147.1,132.7,130.0,123.5,102.7,100.6,-0.3$. The characterization data corresponded to the reported values. ${ }^{[1]}$

\section{1-[4-Nitrophenylethynyl]-1,2-benziodoxol-3(1H)-one (9)}<smiles>O=C1OI(O)c2ccccc21</smiles>

14<smiles>C[SiH2]C#Cc1ccc([N+](=O)[O-])cc1</smiles>

38

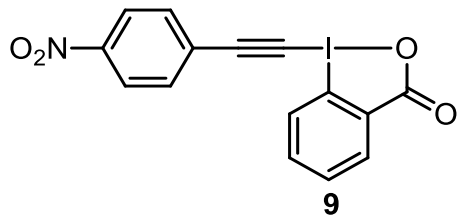

Trimethylsilyl triflate (763 $\mu \mathrm{L}, 4.15 \mathrm{mmol}, 1.10$ equiv) was added to a suspension of 2 iodosylbenzoic acid (14) $\left(1.00 \mathrm{~g}, 3.77 \mathrm{mmol}, 1.00\right.$ equiv) in $\mathrm{CH}_{2} \mathrm{Cl}_{2}(10 \mathrm{~mL})$ at RT. The resulting yellow mixture was stirred for $1 \mathrm{~h}$, followed by the dropwise addition of ((4nitrophenyl)ethynyl)trimethylsilane (38) (1.01 g, $4.15 \mathrm{mmol}, 1.10 \mathrm{equiv})$. The resulting suspension was stirred for $6 \mathrm{~h}$ at RT. A saturated solution of $\mathrm{NaHCO}_{3}(15 \mathrm{~mL})$ was then added and the mixture was stirred vigorously. The resulting suspension was filtered, washed with acetone and dried under vacuum to afford 9 (864 $\mathrm{mg}, 2.19 \mathrm{mmol}, 59 \%)$ as a pale brown solid.

Mp (Dec.) $142-151{ }^{\circ} \mathrm{C} .{ }^{1} \mathrm{H}$ NMR $\left(400 \mathrm{MHz}, \mathrm{CDCl}_{3}\right)($ ca $0.01 \mathrm{mmol} / \mathrm{ml}) \delta 8.44(\mathrm{~m}, 1 \mathrm{H}$; $\operatorname{Ar} H), 8.31$ (m, $2 \mathrm{H} ; \operatorname{Ar} H), 8.23(\mathrm{~m}, 1 \mathrm{H} ; \mathrm{ArH}), 7.74-7.86(\mathrm{~m}, 4 \mathrm{H} ; \mathrm{ArH}) .{ }^{13} \mathrm{C} \mathrm{NMR}(101$ $\left.\mathrm{MHz}, \mathrm{CDCl}_{3}\right) \delta 166.3,148.5,135.2,133.6,132.7,131.9,131.1,127.1,126.2,123.9,116.0$, 103.2, 57.1. IR $\vee 3104(\mathrm{w}), 2432(\mathrm{w}), 2164(\mathrm{w}), 1938(\mathrm{w}), 1614(\mathrm{~s}), 1586(\mathrm{~m}), 1515(\mathrm{~s}), 1403$ (m), 1341 (s), 1305 (s), $1221(\mathrm{w}), 1105$ (w), 1008 (w), 858 (s), 828 (m), 740 (s), 689 (m), 636 (m). HRMS(ESI) calcd for $\mathrm{C}_{15} \mathrm{H}_{9} \mathrm{NO}_{4} \mathrm{I}^{+}(\mathrm{M}+\mathrm{H}) 393.9576$, found 393.9577.

\section{(Mesitylethynyl)trimethylsilane (40)}

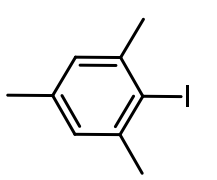

39

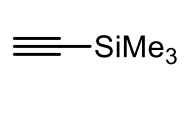

16

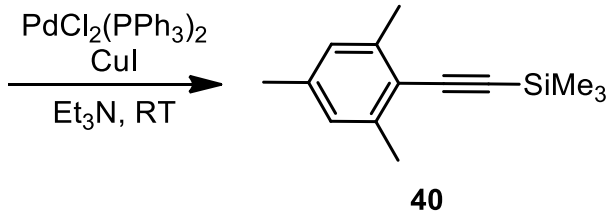

40

Following a slight modification of the reported procedure, ${ }^{[11]}$ aryl iodide 39 (1.05 g, 4.27 mmol, 1.00 equiv) was dissolved in $\mathrm{Et}_{3} \mathrm{~N}(10 \mathrm{~mL})$ (without prior drying). After three freeze-

[11]I. Van Overmeire, S. A. Boldin, S. A.; K. Venkataraman, R. Zisling, S. De Jonghe, S. Van Calenbergh, D. De Keukeleire, A. H. Futerman, P. Herdewijn, J. Med. Chem. 2000, 43, 4189. 
thaw-pump cycles, $\mathrm{PdCl}_{2}\left(\mathrm{PPh}_{3}\right)_{2}(30 \mathrm{mg}, 0.42 \mathrm{mmol}, 0.1$ equiv) and $\mathrm{CuI}(16 \mathrm{mg}, 0.84 \mathrm{mmol}$, 0.20 equiv) were added under $\mathrm{N}_{2}$. After the addition of trimethylsilylacetylene (16) $(1.2 \mathrm{~mL}$, $8.5 \mathrm{mmol}, 2.0$ equiv), the green suspension was stirred at RT for $1 \mathrm{~h}$. The reaction mixture was reduced under vacuum, dissolved in DCM $(30 \mathrm{~mL})$, washed with 5\% EDTA solution and water. Organic layers were then dried over $\mathrm{MgSO}_{4}$, filtered and reduced under vacuum. The resulting oil was purified by column chromatography (PET) to afford 40 (526 mg, $2.43 \mathrm{mmol}$, $66 \%$ ) along with $15 \%$ of starting material. Used without further purification.

$\mathrm{R}_{f} 0.5$ (PET, UV). ${ }^{1} \mathrm{H}$ NMR (400 MHz, $\left.\mathrm{CDCl}_{3}\right) \delta 6.87(\mathrm{~s}, 2 \mathrm{H} ; \mathrm{ArH}), 2.41\left(\mathrm{~s}, 6 \mathrm{H} ; \mathrm{CH}_{3}\right)$, 2.29 (s, $\left.3 \mathrm{H} ; \mathrm{CH}_{3}\right), 0.28$ (s, $\left.9 \mathrm{H} ; \mathrm{Si}\left(\mathrm{CH}_{3}\right)_{3}\right)$.

\section{1-[2,4,6-Trimethylphenylethynyl]-1,2-benziodoxol-3(1H)-one (10)}

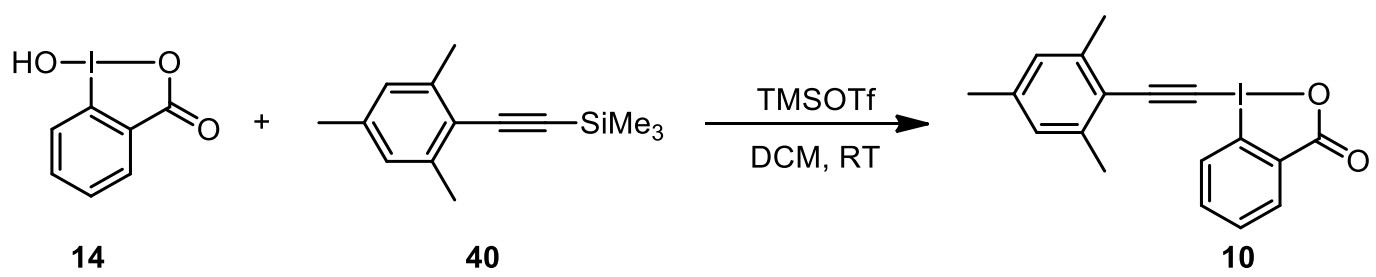

Trimethylsilyl triflate $(212 \mu \mathrm{L}, 1.15 \mathrm{mmol}, 1.10$ equiv) was added to a suspension of 2 iodosylbenzoic acid (14) (1.00 g, $1.05 \mathrm{mmol}, 1.00$ equiv) in $\mathrm{CH}_{2} \mathrm{Cl}_{2}(4 \mathrm{~mL})$ at RT. The resulting yellow mixture was stirred for $1 \mathrm{~h}$, followed by the dropwise addition of (mesitylethynyl)trimethylsilane (40) (250 mg, $1.15 \mathrm{mmol}, 1.10$ equiv) dissolved in DCM (1 $\mathrm{mL})$. The resulting suspension was stirred for $6 \mathrm{~h}$ at RT. A saturated solution of $\mathrm{NaHCO}_{3}(5$ $\mathrm{mL}$ ) was then added and the mixture was stirred vigorously. The layers were separated and the organic layer was washed with sat. $\mathrm{NaHCO}_{3}$, dried over $\mathrm{MgSO}_{4}$, filtered and evaporated under reduced pressure. The resulting solid was recrystallized in $\mathrm{CH}_{3} \mathrm{CN}$ (ca $20 \mathrm{~mL}$ ). The mother liqueurs were reduced and recrystallized in $\mathrm{CH}_{3} \mathrm{CN}(4 \mathrm{~mL})$. Both solids were combined, washed with pentane and dried under high vacuum to afford $\mathbf{1 0}$ (120 mg, 0.310 mmol, $30 \%$ ) as a tan solid.

$\mathrm{Mp}$ (Dec.) $171-175{ }^{\circ} \mathrm{C} .{ }^{1} \mathrm{H}$ NMR $\left(400 \mathrm{MHz}, \mathrm{CDCl}_{3}\right)(\mathrm{ca} 0.01 \mathrm{mmol} / \mathrm{ml}) \delta 8.38(\mathrm{~m}, 1 \mathrm{H}$; $\operatorname{ArH}), 8.28$ (m, $1 \mathrm{H} ; \operatorname{Ar} H), 7.72(\mathrm{~m}, 2 \mathrm{H} ; \mathrm{ArH}), 6.92$ (s, $2 \mathrm{H}$; MesH), 2.45 (s, $\left.6 \mathrm{H} ; \mathrm{CH}_{3}\right), 2.31$ $\left(\mathrm{s}, 3 \mathrm{H} ; \mathrm{CH}_{3}\right) .{ }^{13} \mathrm{C}$ NMR $\left(101 \mathrm{MHz}, \mathrm{CDCl}_{3}\right) \delta 166.7,142.1,140.5,134.5,132.2,131.5,131.3$, 128.0, 126.2, 117.5, 116.5, 105.1, 55.6, 21.4, 21.0. IR v $2979(\mathrm{w}), 2916(\mathrm{w}), 2247(\mathrm{w}), 2131$ (w), $1650(\mathrm{~m}), 1623(\mathrm{~m}), 1562(\mathrm{w}), 1439(\mathrm{w}), 1333(\mathrm{w}), 1292(\mathrm{w}), 1212(\mathrm{w}), 1146(\mathrm{w}), 1008$ (w), 906 (s), 855 (w), 833 (w), 729 (s), 647 (m). HRMS(ESI) calcd for $\mathrm{C}_{18} \mathrm{H}_{16} \mathrm{O}_{2} \mathrm{I}^{+}(\mathrm{M}+\mathrm{H})$ 391.0195 , found 391.0191 .

\subsection{Preparation of Substrates and Catalysts}

Methyl 1-oxo-2-indanecarboxylate (2a)<smiles>O=C1CCc2ccccc21</smiles>

41

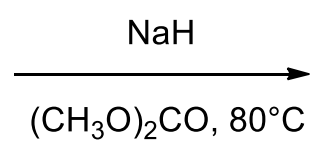

$\left(\mathrm{CH}_{3} \mathrm{O}\right)_{2} \mathrm{CO}, 80^{\circ} \mathrm{C}$<smiles>COC(=O)C1Cc2ccccc2C1=O</smiles>

$2 a$ 
Following the reported procedure, ${ }^{[12]}$ a suspension of $\mathrm{NaH}(6.64 \mathrm{~g}, 60 \%$ in mineral oil, 166 mmol, 2.2 equiv) in dimethyl carbonate $(20 \mathrm{~mL})$ was charged in a $250 \mathrm{~mL}$ two-neck flask. 1Indanone (41) (10.0 g, $75.5 \mathrm{mmol}, 1$ equiv) in dimethyl carbonate $(70 \mathrm{~mL})$ was added dropwise and the resulting mixture was refluxed at $80{ }^{\circ} \mathrm{C}$ for $2 \mathrm{~h}$. After cooling to RT, 200 $\mathrm{mL}$ of water was added. The aqueous layer was separated and extracted with $\mathrm{CH}_{2} \mathrm{Cl}_{2}(3 \times 50$ $\mathrm{mL}$ ). The combined organic extracts were dried over $\mathrm{MgSO}_{4}$ filtered and concentrated under reduce pressure. The brown residual oil was purified by flash chromatography (PET/EtOAc 4:1) to afford $2 \mathbf{a}(10.5 \mathrm{~g}, 55.4 \mathrm{mmol}, 73 \%)$ as orange solid.

Mp (Dec.) 139-145 ${ }^{\circ}$; R 0.3 (PET/EtOAc 4:1, UV/Anisaldehyde); ${ }^{1} \mathrm{H}$ NMR (400 MHz, $\mathrm{CDCl}_{3}$ ) Keto-enol (6:1) $\delta 10.35$ (br s, $0.16 \mathrm{H}$; OH-enol), ), $7.76(\mathrm{~d}, J=7.6 \mathrm{~Hz}, 1 \mathrm{H}$; $\mathrm{ArH}$ ), $7.61(\mathrm{t}, J=7.5 \mathrm{~Hz}, 1 \mathrm{H}$; $\mathrm{Ar} H), 7.49(\mathrm{~d}, J=7.7 \mathrm{~Hz}, 1 \mathrm{H}$; ArH), 7.38 (t, $J=7.5 \mathrm{~Hz}, 1 \mathrm{H}$; ArH), 3.84 (s, $0.5 \mathrm{H}$; OCH 3 -enol), 3.78 (s, $3 \mathrm{H} ; \mathrm{OCH}_{3}$ ), 3.73 (dd, $\left.J=8.2,4.0 \mathrm{~Hz}, 1 \mathrm{H} ; \mathrm{CHCO}\right), 3.55$ (dd, $J=17.3,3.8 \mathrm{~Hz}, 1 \mathrm{H}$; $\mathrm{CH}_{2}$ ), 3.50 (s, $0.3 \mathrm{H}$; $\mathrm{CH}_{2}$-enol), 3.37 (dd, $J=17.3,8.3 \mathrm{~Hz}, 1 \mathrm{H}$; $\left.\mathrm{CH}_{2}\right) ;{ }^{13} \mathrm{C} \mathrm{NMR}\left(101 \mathrm{MHz}, \mathrm{CDCl}_{3}\right) \delta 199.3,169.4,153.5,135.3,135.0,129.3,127.7,126.7$, 126.4, 124.6, 124.5, 120.6, 53.0, 51.1, 32.4, 30.1; IR v 3033 (w), 2954 (w), 2848 (w), 2360 (w), 2342 (w), 1742 (s), $1711(\mathrm{~s}), 1653$ (m), 1609 (m), $1574(\mathrm{~m}), 1464$ (m), 1436 (m), 1328 (m), 1298 (m), 1261 (m), 1209 (s), 1154 (s), 1095 (w), 989 (m), 963 (w), 850 (w), 764 (s), $722(\mathrm{w}), 683(\mathrm{w})$. Characterization data of $\mathbf{2 a}$ corresponded to the literature values. ${ }^{[12]}$

\section{Tert-Butyl 1-oxoindan-2-carboxylate (2b)}

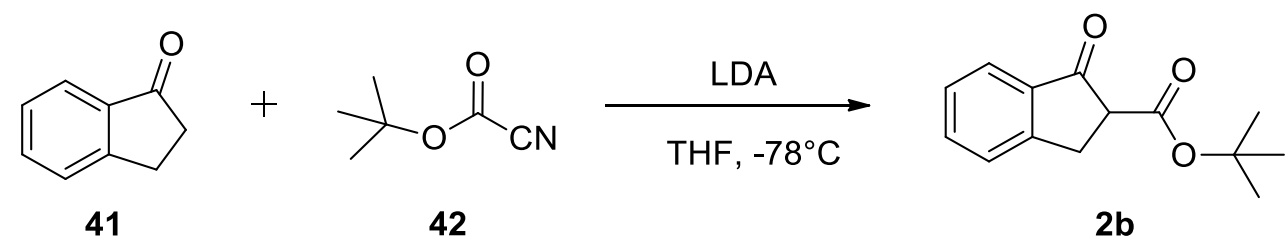

Following the reported procedure ${ }^{[13]} n$-butyllithium $(2.5 \mathrm{M}, 3.30 \mathrm{~mL}, 8.32 \mathrm{mmol}, 1.1$ equiv) was slowly added to a cooled $\left(-78^{\circ} \mathrm{C}\right)$ mixture of diisopropylamine $(1.17 \mathrm{~mL}, 8.32$ mmol, 1.1 equiv) in dried THF (12 mL). The resulting mixture was placed in an ice-bath and stirred at $0{ }^{\circ} \mathrm{C}$ for $0.5 \mathrm{~h}$. After this time, the mixture was cooled at $-78{ }^{\circ} \mathrm{C}$ and a solution of 1 indanone (41) (1.00 g, $7.56 \mathrm{mmol}, 1$ equiv) in THF (15 mL) was added and stirred $30 \mathrm{~min}$ at $78{ }^{\circ} \mathrm{C}$. tert-Butyl cyanoformate $(\mathbf{4 2})(1.06 \mathrm{~g}, 8.32 \mathrm{mmol}, 1.1$ equiv) in THF (5 mL) was added. The resulting mixture was stirred $1 \mathrm{~h}$ at $-78{ }^{\circ} \mathrm{C}$, and then allowed to warm to RT. The reaction was quenched with sat. $\mathrm{NH}_{4} \mathrm{Cl}$ solution, the aqueous layer was extracted with $\mathrm{Et}_{2} \mathrm{O}$ and the combined organic layers were dried over $\mathrm{Na}_{2} \mathrm{SO}_{4}$, filtered and evaporated under reduce pressure. The resulting crude mixture was purified by flash chromatography (Hexane/EtOAc, 95:5) to give $\mathbf{2 b}$ as pink oil (663 mg, $2.85 \mathrm{mmol}, 40 \%$ ).

$\mathrm{R}_{f} 0.6$ (PET/EtOAc 4:1, UV/Anisaldehyde); ${ }^{1} \mathrm{H}$ NMR (400 MHz, $\left.\mathrm{CDCl}_{3}\right) \delta 7.73(\mathrm{~d}, J=7.7$ $\mathrm{Hz}, 1 \mathrm{H} ; \operatorname{Ar} H), 7.58(\mathrm{t}, J=7.2 \mathrm{~Hz}, 1 \mathrm{H} ; \operatorname{Ar} H), 7.47(\mathrm{~d}, J=7.5 \mathrm{~Hz}, 1 \mathrm{H} ; \operatorname{Ar} H), 7.35(\mathrm{t}, J=7.2 \mathrm{~Hz}, 1 \mathrm{H}$; $\operatorname{Ar} H$ ), $3.60\left(\mathrm{dd}, J=8.2,4.0 \mathrm{~Hz}, 1 \mathrm{H}\right.$; CHCO), 3.47 (dd, $\left.J=17.2,3.9 \mathrm{~Hz}, 1 \mathrm{H} ; \mathrm{CH}_{2}\right), 3.31$ (dd, $1 \mathrm{H}, J=$ 17.2, 8.2 Hz, $\left.1 \mathrm{H} ; \mathrm{CH}_{2}\right), 1.47$ (s, 9H; $\left.\mathrm{OC}\left(\mathrm{CH}_{3}\right)_{3}\right) ;{ }^{13} \mathrm{C} \mathrm{NMR}\left(101 \mathrm{MHz}, \mathrm{CDCl}_{3}\right) \delta 199.9,168.2,153.6$,

[12]K. Van Emelen, T. De Wit, G. J. Hoornaert, F. Compernolle, Tetrahedron. 2002, 58, 4225.

[13]T. B. Poulsen, L. Bernardi, J. Aleman, J. Overgaard, K. A. Jorgensen, J. Am. Chem. Soc. 2007, $129,441$. 
135.3, 135.1, 127.6, 126.4, 124.4, 81.9, 54.3, 30.2, 27.9; IR 3073 (w), 2979 (w), 2933 (w), 2870 (w), 2359 (w), 2342 (w), 1713 (s), 1645 (w), 1608 (w), 1574 (w), 1464 (w), 1393 (w), 1369 (m), 1329 (w), 1293 (w), 1270 (w), 1254 (w), 1211 (w), 1149 (s), 1092 (w), 1014 (w), 989 (w), 897 (w), 845 (w), 773 $(\mathrm{w}), 759(\mathrm{w}), 740(\mathrm{~m}), 680(\mathrm{w}), 630(\mathrm{w})$; Characterization data of $\mathbf{4 b}$ corresponded to the literature values. ${ }^{[14]}$

General procedure 1 (GP1): Following the reported procedure, ${ }^{[15]}$ methyl 1-oxo-2indanecarboxylate (2a) (1 equiv), the corresponding alcohol, the transesterification catalyst DMAP or ZnO and toluene or cyclohexane were charged into a flask equipped with a DeanStark trap and reflux condenser. The reaction mixture was heated to reflux, distilling the methanol formed during the reaction. The mixture was refluxed until completed conversion and then volatiles were evaporated under reduced pressure. The obtained crude residues were purified by column chromatography.

$\alpha, \alpha$-Dimethylbenzyl 1-indanone-2-carboxylate (2c)<smiles>COC(=O)C1Cc2ccccc2C1=O</smiles>

$2 \mathrm{a}$<smiles>CC(C)(O)c1ccccc1</smiles>

43

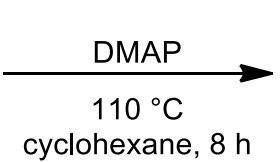

cyclohexane, $8 \mathrm{~h}$

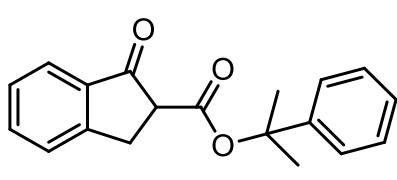

2c

Following the general procedure GP1, $\beta$-keto ester $\mathbf{2 a}$ (1.00 g, $5.26 \mathrm{mmol}, 1.00$ equiv), 2 phenyl-2-propanol (43) (1.22 g, $8.94 \mathrm{mmol}, 1.70$ equiv) and DMAP (50 mg, $4.1 \mathrm{mmol}, 0.078$ equiv) were dissolved in cyclohexane $(50 \mathrm{~mL})$. The reaction mixture was heated at reflux for $8 \mathrm{~h}$. The resulting crude residue was purified by flash chromatography (Pentane/AcOEt 10:1) to afford 2c (464 mg, $1.57 \mathrm{mmol}, 30 \%$ ) as pink oil.

$\mathrm{R}_{f} 0.6$ (PET/EtOAc 4:1, UV/Anisaldehyde). ${ }^{1} \mathrm{H}$ NMR (400 MHz, $\mathrm{CDCl}_{3}$ ) Keto-enol (6:1) $\delta 10.35$ (br s, $0.16 \mathrm{H}$; OH-enol), $7.78(\mathrm{~d}, J=7.7 \mathrm{~Hz}, 1 \mathrm{H} ; \mathrm{Ar} H), 7.61(\mathrm{t}, J=7.6 \mathrm{~Hz}, 1 \mathrm{H}$; $\operatorname{ArH}), 7.36$ (m, $7 \mathrm{H}$; $\mathrm{ArH}$ ), 3.73 (dd, $J=8.2,4.0 \mathrm{~Hz}, 1 \mathrm{H}$; CHCO), 3.61 (s, $2 \mathrm{H}$; CH -enol), $3.51\left(\mathrm{dd}, J=17.2,3.9 \mathrm{~Hz}, 1 \mathrm{H}\right.$; $\left.\mathrm{CH}_{2}\right), 3.33\left(\mathrm{dd}, J=17.2,8.2 \mathrm{~Hz}, 1 \mathrm{H} ; \mathrm{CH}_{2}\right), 1.89$ (s, $0.97 \mathrm{H}$; $\left(\mathrm{CH}_{3}\right)_{2}$-enol), 1.83 (s, $\left.3 \mathrm{H} ; \mathrm{CH}_{3}\right), 1.81$ (s, $\left.3 \mathrm{H} ; \mathrm{CH}_{3}\right) .{ }^{13} \mathrm{C} \mathrm{NMR}\left(101 \mathrm{MHz}, \mathrm{CDCl}_{3}\right) \delta$ 199.6, 167.3, 153.6, 145.3, 135.4, 135.3, 128.2, 127.7, 127.0, 126.5, 124.6, 124.3, 83.2, 54.1, 30.1, 28.8, 28.3, minor peaks observed for enol form 129.2, 128.4, 126.8, 124.1, 120.6, 32.8, 29.1. IR $v 3063(\mathrm{w}), 3032(\mathrm{w}), 2981(\mathrm{w}), 2930$ (w), $1741(\mathrm{w}), 1713(\mathrm{~s}), 1643(\mathrm{w}), 1607$ (w), 1573 (w), 1497 (w), 1449 (w), 1384 (w), 1273 (w), 1209 (m), 1138 (m), 1102 (w), 1032 (w), 988 (w), $912(\mathrm{w}), 765(\mathrm{~m}), 657(\mathrm{~s}), 642(\mathrm{~s})$. The characterization data corresponded to the reported values. ${ }^{[15]}$

1-Adamantyl 1-indanone-2-carboxylate (2d)<smiles>COC(=O)C1Cc2ccccc2C1=O</smiles>

$2 a$<smiles>OC1(C2CC3CCC2CC3)CC2CCC1C2</smiles>

44<smiles>O=C(OC12CC3CC(CC(C3)C1)C2)C1Cc2ccccc2C1=O</smiles>

2d

[14]T. A. Moss, D. R. Fenwick, D. J. Dixon, J. Am. Chem. Soc. 2008, 130, 10076.

[15]M. Lian, Z. Li, J. Du, Q. Meng, Z. Gao, Eur. J. Org. Chem. 2010, 2010, 6525. 
Following the general procedure GP1, $\beta$-keto ester $2 \mathbf{a}$ ( $2.00 \mathrm{~g}, 10.5 \mathrm{mmol}, 1.00$ equiv), 1 adamantanol (44) (2.07 g, $13.6 \mathrm{mmol}, 1.30$ equiv) and $\mathrm{ZnO}$ (176 mg, $2.16 \mathrm{mmol}, 0.20$ equiv) were dissolved in toluene $(10 \mathrm{~mL})$. The reaction mixture was heated at reflux for $18 \mathrm{~h}$. The resulting crude residue was purified by flash chromatography (Hexane/AcOEt 20:1) to afford 2d (990 $\mathrm{mg}, 3.20 \mathrm{mmol}, 30 \%)$ as pink powder.

$\mathrm{R}_{f} 0.6$ (PET/EtOAc 4:1, UV/Anisaldehyde). ${ }^{1} \mathrm{H}$ NMR (400 MHz, $\mathrm{CDCl}_{3}$ ) Keto-enol (6:1) $\delta 10.59$ (br s, $0.11 \mathrm{H}$; OH-enol), $\delta 7.76$ (d, $J=7.6 \mathrm{~Hz}, 1 \mathrm{H}$; $\operatorname{ArH}$ ), 7.60 (m, $1 \mathrm{H} ; \operatorname{Ar} H), 7.49$ $(\mathrm{d}, J=7.7 \mathrm{~Hz}, 1 \mathrm{H} ; \mathrm{ArH}), 7.38(\mathrm{~m}, 1 \mathrm{H} ; \mathrm{ArH}), 3.61(\mathrm{dd}, J=8.2,4.0 \mathrm{~Hz}, 1 \mathrm{H} ; \mathrm{CHCO}), 3.49$ (m, $\left.1 \mathrm{H} ; \mathrm{CH}_{2}\right), 3.33$ (dd, $\left.J=17.2,8.2 \mathrm{~Hz}, 1 \mathrm{H} ; \mathrm{CH}_{2}\right), 2.23$ (s, $2 \mathrm{H} ; \mathrm{CHCH}$-enol), 2.14 (s, $9 \mathrm{H}$; $\mathrm{CHCH}_{2}$ ), 1.71 (s, $1 \mathrm{H} ; \mathrm{CHCH}_{3}$-enol), 1.65 (s, $\left.6 \mathrm{H} ; \mathrm{CHCH}_{2}\right) .{ }^{13} \mathrm{C} \mathrm{NMR}\left(101 \mathrm{MHz}, \mathrm{CDCl}_{3}\right) \delta$ 200.0, 167.9, 153.7, 135.5, 135.2, 127.6, 126.5, 124.5, 82.1, 54.5, 41.2, 36.1, 30.8, minor peaks observed for enol form 129.0, 126.6, 120.5, 82.1, 45.3, 41.8, 32.9, 30.4. IR v $3735(\mathrm{w})$, 3709 (w), 3645 (w), 3034 (w), 2912 (m), 2852 (w), 2680 (w), 1733 (m), 1713 (s), $1645(\mathrm{w})$, $1610(\mathrm{w}), 1573(\mathrm{~m}), 1462(\mathrm{w}), 1353(\mathrm{w}), 1289(\mathrm{~m}), 1259(\mathrm{~m}), 1208(\mathrm{~m}), 1188(\mathrm{~m}), 1164(\mathrm{~m})$, $1090(\mathrm{~m}), 1056(\mathrm{~m}), 984(\mathrm{w}), 984(\mathrm{w}), 933$ (w), $910(\mathrm{w}), 874(\mathrm{w}), 814(\mathrm{w}), 763(\mathrm{~m}), 722(\mathrm{w})$, $652(\mathrm{~s}), 630(\mathrm{~s})$. The characterization data corresponded to the reported values. ${ }^{[15]}$

\section{9-Anthracenemethyl 1-indanone-2-carboxylate (2e)}

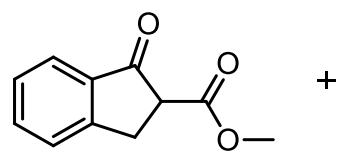

$2 \mathbf{a}$<smiles>OCc1c2ccccc2cc2ccccc12</smiles>

45

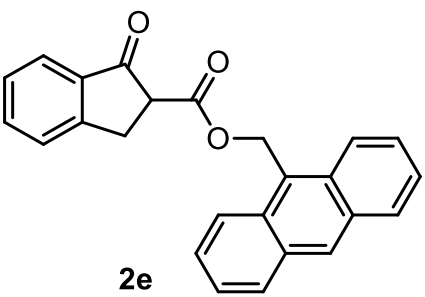

$2 e$

Following the general procedure GP1, $\beta$-keto ester 2a (1.00 g, $5.26 \mathrm{mmol}, 1.00$ equiv), 9anthracenemethanol (45) (1.64 g, $7.89 \mathrm{mmol}, 1.50$ equiv) and DMAP (50 mg, $4.1 \mathrm{mmol}$, 0.078 equiv) were dissolved in cyclohexane $(50 \mathrm{~mL})$. The reaction mixture was heated at reflux for $4.5 \mathrm{~h}$. The resulting crude residue was purified by flash chromatography (Pentane/AcOEt 5:1) to afford $\mathbf{2 e}(960 \mathrm{mg}, 2.62 \mathrm{mmol}, 50 \%)$ as a yellow powder.

$\mathrm{R}_{f} 0.33$ (PET/EtOAc 4:1, UV/Anisaldehyde). ${ }^{1} \mathrm{H}$ NMR (400 MHz, DMSO) Keto-enol (5:1) $\delta 10.71$ (br s, $0.2 \mathrm{H}$; OH-enol), 8.73 (s, $1 \mathrm{H}$; $\operatorname{Ar} H$ ), 8.54 (d, $J=8.8 \mathrm{~Hz}, 1 \mathrm{H}$; $\operatorname{ArH}), 8.37$ $(\mathrm{d}, J=8.7 \mathrm{~Hz}, 2 \mathrm{H}$; ArH), $8.15(\mathrm{~d}, J=8.3 \mathrm{~Hz}, 2 \mathrm{H}$; $\operatorname{Ar} H), 7.76-7.30(\mathrm{~m}, 7 \mathrm{H}$; ArH), 6.31 (s, $0.4 \mathrm{H}$; OCH $\mathrm{OH}_{2}$-enol), 6.24 - 6.17 (m, $2 \mathrm{H}$; OCH $\mathrm{OCH}_{2}, 3.92$ (dd, $J=7.6,4.5 \mathrm{~Hz}, 1 \mathrm{H} ; \mathrm{CHCO}$ ), 3.44 3.31 (m, 2H; $\mathrm{CH}_{2}$ ), 2.51 (s, $0.4 \mathrm{H}$; $\mathrm{CH}_{2}$-enol). ${ }^{13} \mathrm{C}$ NMR (101 MHz, DMSO) $\delta 199.6,169.5$, 153.8, 135.6, 130.9, 130.5, 129.0, 128.9, 127.8, 126.8, 126.7, 125.3, 124.0, 123.8, 59.5, 52.9, $39.5,30.3, \delta 137.4,134.8,130.9,129.0,128.8,126.7,125.9,102.2,57.7,33.2$. IR $\vee 3685(\mathrm{w})$, 3673 (w), 3659 (w), 2987 (s), 2971 (s), 2902 (s), 2367 (w), 1738 (w), 1715 (w), 1652 (w), 1625 (w), 1573 (w), 1452 (w), 1407 (m), $1394(\mathrm{~m}), 1382(\mathrm{~m}), 1252$ (m), $1231(\mathrm{w}), 1078(\mathrm{~s})$, $1057(\mathrm{~s}), 893(\mathrm{w}), 870(\mathrm{w}), 763(\mathrm{w}), 735(\mathrm{w}), 722(\mathrm{w}), 649(\mathrm{w}), 633(\mathrm{w})$. The characterization data corresponded to the reported values. ${ }^{[15]}$

1-Oxo- $N$-phenyl-2,3-dihydro-1H-indene-2-carboxamide (2f) 
<smiles>O=C1CCc2ccccc21</smiles>

41<smiles>O=C=Nc1ccccc1</smiles>

46

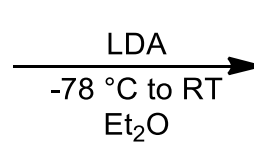

$\mathrm{t}_{2} \mathrm{O}$

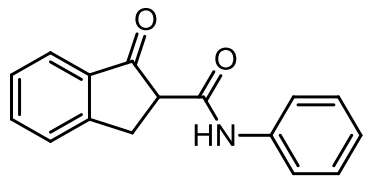

$2 f$

Following a slightly modified reported procedure, ${ }^{[16]} n$-butyllithium $(2.5 \mathrm{M}$ in hexane, 6.05 $\mathrm{mL}, 15.1 \mathrm{mmol}, 1.00$ equiv) was slowly added to a cooled $\left(-78{ }^{\circ} \mathrm{C}\right)$ mixture of diisopropylamine (2.14 mL, $15.1 \mathrm{mmol}, 1.00$ equiv) in dried $\mathrm{Et}_{2} \mathrm{O}(60 \mathrm{~mL})$. Once the addition was finished, an additional quantity of $\mathrm{Et}_{2} \mathrm{O}(30.3 \mathrm{~mL})$ was added and the final mixture was stirred at $-78{ }^{\circ} \mathrm{C}$ for $0.5 \mathrm{~h}$. After this time, a solution of 1-indanone (41) $(2.00 \mathrm{~g}, 15.1 \mathrm{mmol}$, 1.00 equiv) in $\mathrm{Et}_{2} \mathrm{O}(15 \mathrm{~mL})$ was added and the mixture was stirred $30 \mathrm{~min}$ at $-78{ }^{\circ} \mathrm{C}$. Phenyl isocyanate (46) $\left(1.65 \mathrm{~mL}, 15.1 \mathrm{mmol}, 1.00\right.$ equiv) in $\mathrm{Et}_{2} \mathrm{O}(15 \mathrm{~mL})$ was added by cannula and the resulting mixture was stirred and allowed to warm to RT for $2 \mathrm{~h}$. The reaction was quenched with sat. $\mathrm{NH}_{4} \mathrm{Cl}$ solution and the aqueous layer was extracted with $\mathrm{CH}_{2} \mathrm{Cl}_{2}$. The combined organic layers were washed with $\mathrm{H}_{2} \mathrm{O}$ and dried over $\mathrm{Na}_{2} \mathrm{SO}_{4}$, filtered and evaporated under reduced pressure. The resulting crude mixture was purified by flash chromatography (Hexane/EtOAc, 20:1) to give $\mathbf{2 f}$ as a colorless solid (1.00 g, $3.98 \mathrm{mmol}$, $26 \%)$.

$\mathrm{Mp}$ (Dec.) 170-181 ${ }^{\circ} \mathrm{C}$ (lit 168-177 ${ }^{\circ} \mathrm{C}$ ). $\mathrm{R}_{f} 0.63$ (PET/EtOAc 4:1, UV/Anisaldehyde). ${ }^{1} \mathrm{H}$ NMR (400 MHz, $\left.\mathrm{CDCl}_{3}\right) \delta 9.18($ br s, $1 \mathrm{H} ; \mathrm{NH}), 7.80(\mathrm{~d}, J=7.9 \mathrm{~Hz}, 1 \mathrm{H} ; \mathrm{ArH}), 7.67(\mathrm{~m}, 1 \mathrm{H}$; $\operatorname{ArH}), 7.58(\mathrm{~m}, 3 \mathrm{H}$; $\operatorname{ArH}), 7.42(\mathrm{~m}, 1 \mathrm{H} ; \mathrm{ArH}), 7.34$ (m, $2 \mathrm{H}$; $\operatorname{ArH}), 7.12(\mathrm{~m}, 1 \mathrm{H} ; \mathrm{ArH}), 3.86$ $\left(\mathrm{m}, 1 \mathrm{H} ; \mathrm{CH}_{2}\right), 3.75(\mathrm{dd}, J=8.2,4.1 \mathrm{~Hz}, 1 \mathrm{H} ; \mathrm{CHCO}), 3.45\left(\mathrm{dd}, J=17.8,8.2 \mathrm{~Hz}, 1 \mathrm{H} ; \mathrm{CH}_{2}\right)$. ${ }^{13} \mathrm{C}$ NMR $\left(101 \mathrm{MHz}, \mathrm{CDCl}_{3}\right) \delta 203.5,164.3,154.2,136.1,129.0,127.8,126.7,124.5,124.3$, 119.9, 53.3, 28.5. IR v $3684(\mathrm{w}), 3665(\mathrm{w}), 3318(\mathrm{w}), 2987(\mathrm{~s}), 2971(\mathrm{~s}), 2902(\mathrm{~s}), 1715(\mathrm{~s})$, $1706(\mathrm{w}), 1682(\mathrm{w}), 1652(\mathrm{~m}), 1599(\mathrm{~m}), 1534(\mathrm{~m}), 1501(\mathrm{w}), 1463(\mathrm{w}), 1444(\mathrm{~m}), 1407(\mathrm{w})$, 1394 (w), 1348 (w), 1273 (w), 1249 (m), 1148 (w), 1077 (s), 1066 (s), 1057 (s), 900 (w), 868 (w), $765(\mathrm{~m}), 744(\mathrm{~m}), 691(\mathrm{w}), 636(\mathrm{w}) . \mathrm{HRMS}(\mathrm{ESI})$ calcd for $\mathrm{C}_{16} \mathrm{H}_{14} \mathrm{NO}_{2}{ }^{+}(\mathrm{M}+\mathrm{H}) 252.1024$, found 252.1028. Two carbons are unresolved.

\section{$N$-(4-Fluorophenyl)-1-oxo-2,3-dihydro-1H-indene-2-carboxamide (2g)}<smiles>COC(=O)C1Cc2ccccc2C1=O</smiles>

$\mathbf{2 a}$

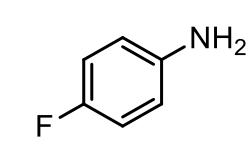

47

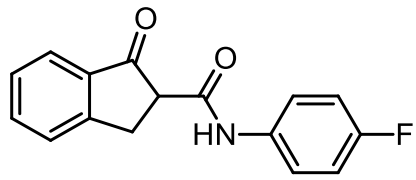

$2 \mathrm{~g}$

Following a slightly modified procedure, ${ }^{[16]}$ methyl 1-oxo-2-indanecarboxylate (2a) $(500$ $\mathrm{mg}, 2.63 \mathrm{mmol}, 1.00$ equiv) was dissolved in xylene $(26 \mathrm{~mL})$. After $5 \mathrm{~min}$ of stirring, 4fluoroaniline (47) $(379 \mu \mathrm{L}, 3.94 \mathrm{mmol}, 1.50$ equiv) was added and the final mixture was refluxed under nitrogen for $18.5 \mathrm{~h}$. The solvent was evaporated under reduced pressure and the residual crude was purified by flash chromatography (Hexane/AcOEt 6:1 to 4:1) to afford $2 \mathrm{~g}$ (164 mg, $0.609 \mathrm{mmol}, 23 \%)$ as a colorless solid.

$\mathrm{R}_{f} 0.63$ (PET/EtOAc 1:1, UV/Anisaldehyde). ${ }^{1} \mathrm{H}$ NMR (400 MHz, $\left.\mathrm{CDCl}_{3}\right) \delta 9.16$ (br s, 1 $\mathrm{H} ; \mathrm{NH}), 7.80(\mathrm{~d}, J=7.7 \mathrm{~Hz}, 1 \mathrm{H} ; \operatorname{ArH}), 7.68(\mathrm{~m}, 1 \mathrm{H} ; \operatorname{Ar} H), 7.55(\mathrm{~m}, 3 \mathrm{H} ; \operatorname{Ar} H), 7.42$ (m, 1 $\mathrm{H} ; \mathrm{ArH}), 7.03(\mathrm{t}, J=8.5 \mathrm{~Hz}, 2 \mathrm{H} ; \mathrm{Ar} H), 3.86\left(\mathrm{dd}, J=17.8,4.2 \mathrm{~Hz}, 1 \mathrm{H} ; \mathrm{CH}_{2}\right), 3.74$ (dd, $J=$ 8.3, 4.2 Hz, $1 \mathrm{H}$; CHCO), $3.45\left(\mathrm{dd}, 1 \mathrm{H}, J=17.9,8.3 \mathrm{~Hz}, 1 \mathrm{H} ; \mathrm{CH}_{2}\right) .{ }^{13} \mathrm{C}$ NMR $(101 \mathrm{MHz}$,

[16]C. Pan, X. Zeng, Y. Guan, X. Jiang, L. Li, H. Zhang, Synlett 2011, 2011, 425, 429. 
$\left.\mathrm{CDCl}_{3}\right) \delta 203.5,164.2,160.5154 .1,136.2,134.8(\mathrm{~m}), 127.8,126.8,126.4,125.3,124.5$, $121.7,121.5(\mathrm{~d}, J(\mathrm{C}, \mathrm{F})=8.4 \mathrm{~Hz}), 115.6(\mathrm{~d}, J(\mathrm{C}, \mathrm{F})=22.4 \mathrm{~Hz}), 53.2,28.5$. IR $\vee 3422(\mathrm{w}), 3386$ (w), 3314 (w), 3159 (w), 3067 (w), 2953 (w), $2872(\mathrm{w}), 2838$ (w), 2360 (w), 1715 (s), 1652 (m), 1613 (w), 1589 (w), 1538 (m), 1512 (s), 1463 (w), 1409 (w), 1346 (w), 1299 (w), 1273 (w), $1216(\mathrm{~m}), 1156(\mathrm{w}), 1100(\mathrm{w}), 1010(\mathrm{w}), 913(\mathrm{w}), 833(\mathrm{~m}), 787(\mathrm{w}), 748(\mathrm{~m}), 695(\mathrm{w})$, $666(\mathrm{~m}), 637$ (s). HRMS(ESI) calcd for $\mathrm{C}_{16} \mathrm{H}_{13} \mathrm{NO}_{2} \mathrm{~F}^{+}(\mathrm{M}+\mathrm{H}) 270.0930$, found 270.0922 .

$N$-(3,5-Bis(trifluoromethyl)phenyl)-1-oxo-2,3-dihydro-1H-indene-2-carboxamide (2h)

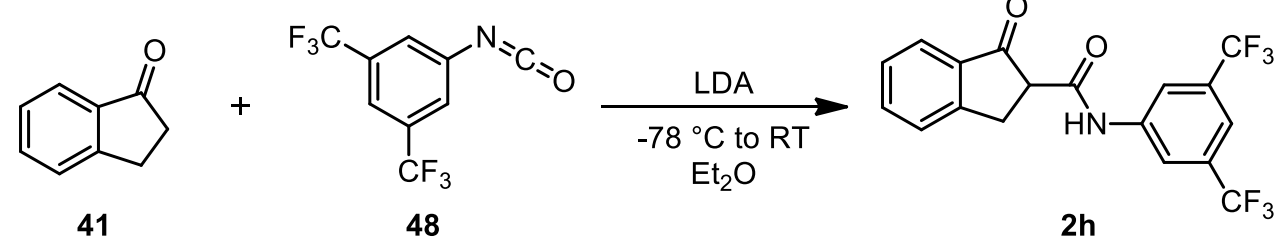

$n$-Butyllithium ( $2.5 \mathrm{M}$ in hexane, $6.05 \mathrm{~mL}, 15.1 \mathrm{mmol}, 1.00$ equiv) was slowly added to a cooled $\left(-78{ }^{\circ} \mathrm{C}\right)$ mixture of diisopropylamine $\left(2.14 \mathrm{~mL}, 15.1 \mathrm{mmol}, 1.00\right.$ equiv) in dried $\mathrm{Et}_{2} \mathrm{O}$ $(60 \mathrm{~mL})$. Once the addition was finished, an additional quantity of $\mathrm{Et}_{2} \mathrm{O}(30.3 \mathrm{~mL})$ was added and the mixture was stirred at $-78{ }^{\circ} \mathrm{C}$ for $0.5 \mathrm{~h}$. After this time, a solution of 1-indanone (41) ( $2.00 \mathrm{~g}, 15.1 \mathrm{mmol}, 1.00$ equiv) in $\mathrm{Et}_{2} \mathrm{O}(15 \mathrm{~mL})$ was added and the mixture was stirred 30 min at $-78{ }^{\circ} \mathrm{C}$. 3,5-Bis(trifluoromethyl)phenyl isocyanate $(48)(2.63 \mathrm{~mL}, 15.1 \mathrm{mmol}, 1.00$ equiv) in $\mathrm{Et}_{2} \mathrm{O}(15 \mathrm{~mL})$ was added by cannula and the resulting mixture was stirred and allowed to warm to RT for $2 \mathrm{~h}$. The reaction was quenched with sat. $\mathrm{NH}_{4} \mathrm{Cl}$ solution and the aqueous layer was extracted with $\mathrm{CH}_{2} \mathrm{Cl}_{2}$. The combined organic layers were washed with $\mathrm{H}_{2} \mathrm{O}$ and dried over $\mathrm{Na}_{2} \mathrm{SO}_{4}$, filtered and evaporated under reduced pressure. The resulting crude mixture was purified by flash chromatography (Hexane/EtOAc, 20:1 to 10:1) to give $\mathbf{2 h}$ as a colorless solid $(1.69 \mathrm{~g}, 4.36 \mathrm{mmol}, 30 \%)$.

$\mathrm{R}_{f} 0.66$ (PET/EtOAc 4:1, UV/Anisaldehyde). ${ }^{1} \mathrm{H}$ NMR (400 MHz, $\left.\mathrm{CDCl}_{3}\right)$ Keto-enol (10:1) $\delta 9.60$ (br s, $1 \mathrm{H}$; NH), 9.07 (br s, $0.10 \mathrm{H}$; NH-enol), 8.10 (s, $2 \mathrm{H}$; ArH), 7.82 (d, $J=$ $7.6 \mathrm{~Hz}, 1 \mathrm{H} ; \operatorname{Ar} H), 7.70$ (t, $J=7.1 \mathrm{~Hz}, 1 \mathrm{H}$; $\operatorname{Ar} H), 7.59$ (m, $2 \mathrm{H} ; \operatorname{Ar} H), 7.45(\mathrm{~m}, 1 \mathrm{H} ; \operatorname{Ar} H)$. $3.82\left(\mathrm{~m}, 2 \mathrm{H}\right.$; $\mathrm{CH}_{2}$ ), 3.49 (dd, $\left.J=17.6,8.0 \mathrm{~Hz}, 1 \mathrm{H} ; \mathrm{CHCO}\right) .{ }^{13} \mathrm{C} \mathrm{NMR}\left(101 \mathrm{MHz}, \mathrm{CDCl}_{3}\right) \delta$ 203.1, 164.9, 154.0, 139.1, 136.5, $132.4(\mathrm{q}, J(\mathrm{C}, \mathrm{F})=33.7 \mathrm{~Hz}), 128.0,126.8,124.7,123.1(\mathrm{q}$, $J(\mathrm{C}, \mathrm{F})=273.0 \mathrm{~Hz}), 119.6(\mathrm{~m}), 117.6(\mathrm{~m}), 53.2,28.3$, minor peaks observed for enol form $\delta$ 136.9, 134.9, 132.6, 132.2, 128.5, 126.8, 126.4, 125.4, 124.4, 121.7, 40.8. HRMS(ESI) calcd for $\mathrm{C}_{18} \mathrm{H}_{12} \mathrm{NO}_{2} \mathrm{~F}_{6}{ }^{+}(\mathrm{M}+\mathrm{H}) 388.0772$, found 388.07748.

\section{$N$-Boc L-valine methyl ester (50)}

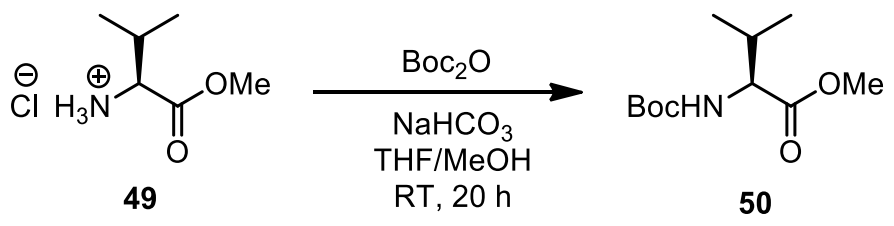

Following the reported procedure, ${ }^{[17]}$ sodium hydrogencarbonate $(7.50 \mathrm{~g}, 89.3 \mathrm{mmol}, 3.00$ equiv) was added in one portion to a stirred solution of L-valine methyl ester hydrochloride (49) $\left(5.00 \mathrm{~g}, 29.8 \mathrm{mmol}, 1.00\right.$ equiv) in dry THF/MeOH $(80 \mathrm{~mL}, 4: 1)$ at $0{ }^{\circ} \mathrm{C}$, followed by the addition of $\mathrm{Boc}_{2} \mathrm{O}(9.75 \mathrm{~g}, 44.7 \mathrm{mmol}, 1.50$ equiv). The final mixture was allowed to warm to

[17]S. E. Gibson, N. Mainolfi, S. B. Kalindjian, P. T. Wright, A. J. P. White, Chem. Eur. J. 2005, 11, 69. 
$\mathrm{RT}$ and stirred for $20 \mathrm{~h}$. The reaction mixture was quenched with water $(55 \mathrm{~mL})$ and extracted with $\mathrm{Et}_{2} \mathrm{O}(2 \times 160 \mathrm{~mL})$. The combined organic layers were washed with saturated aqueous $\mathrm{NaHCO}_{3}$ solution $(2 \times 55 \mathrm{~mL})$, brine $(2 \times 55 \mathrm{~mL})$, dried over $\mathrm{MgSO}_{4}$ and the solvent was removed under reduced pressure. The residual colorless oil was purified by flash chromatography (PET/AcOEt 15:1 to 4:1) to afford the pure product $\mathbf{5 0}(6.60 \mathrm{~g}, 28.5 \mathrm{mmol}$, $96 \%)$ as a colorless oil.

$\mathrm{R}_{f} 0.45$ (PET/AcOEt 6:1, UV/anisaldehyde). ${ }^{1} \mathrm{H}$ NMR (400 MHz, $\left.\mathrm{CDCl}_{3}\right) \delta 5.05$ (br d, $J=$ $8.3 \mathrm{~Hz}, 1 \mathrm{H}$; NH), 4.10 (dd, $J=8.8,4.8 \mathrm{~Hz}, 1 \mathrm{H}$; NHCH), 3.62 (s, $3 \mathrm{H}$; OCH $\mathrm{OH}_{3}, 2.01(\mathrm{~m}, 1 \mathrm{H}$; $\left.\left(\mathrm{CH}_{3}\right)_{2} \mathrm{CH}\right), 1.33\left(\mathrm{~s}, 9 \mathrm{H} ; \mathrm{C}\left(\mathrm{CH}_{3}\right)_{3}\right), 0.84\left(\mathrm{~d}, J=6.8 \mathrm{~Hz}, 3 \mathrm{H} ; \mathrm{CHCH}_{3}\right), 0.78$ (d, $J=6.8 \mathrm{~Hz}, 3$ $\left.\mathrm{H} ; \mathrm{CHCH}_{3}\right) .{ }^{13} \mathrm{C} \mathrm{NMR}\left(101 \mathrm{MHz}, \mathrm{CDCl}_{3}\right) \delta 172.8,155.6,79.5,58.4,51.9,31.2,28.2,18.8$, 17.5. IR v $2968(\mathrm{~m}), 2934(\mathrm{w}), 2878(\mathrm{w}), 2361(\mathrm{w}), 2342(\mathrm{w}), 1743(\mathrm{~m}), 1714(\mathrm{~s}), 1502(\mathrm{~m})$, 1437 (w), 1392 (w), 1367 (s), 1312 (w), 1248 (w), 1206 (w), 1158 (s), $1091(\mathrm{~m}), 1044(\mathrm{~m})$, $1016(\mathrm{~s}), 908$ (m), 869 (m), 839 (w), $781(\mathrm{~s}), 762(\mathrm{w}), 736(\mathrm{w}), 698(\mathrm{w}), 677(\mathrm{w}), 664(\mathrm{w})$, $608(\mathrm{w})$. The characterization data corresponded to the reported values. ${ }^{[17]}$

\section{Preparation of amino alcohols 53 and 54}<smiles>COC(=O)[C@H](NC(=O)OCc1ccccc1)C(C)C</smiles>

50

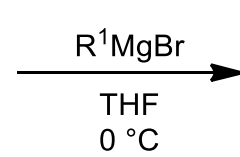<smiles>[R]C([R])(O)[C@H](NC(=O)O)C(C)C</smiles>

51: $\mathrm{R}^{1}=\mathrm{Ph}$ 52: $\mathrm{R}^{1}=p$-Tolyl

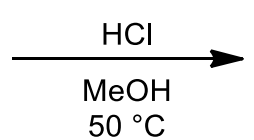

$50{ }^{\circ} \mathrm{C}$

53: $\mathrm{R}^{1}=\mathrm{Ph}$

54: $\mathrm{R}^{1}=p$-Tolyl

General procedure 2 (GP2): Following the reported procedure, ${ }^{[18]}$ a solution of Boc-LVal-OMe (50) (4.00 g, $17.3 \mathrm{mmol}, 1.00$ equiv) in $8.65 \mathrm{~mL}$ of THF was added to a solution of Grignard reagent in THF ( $1 \mathrm{M}, 86.5 \mathrm{mmol}, 5.00$ equiv). The final mixture was stirred for $1 \mathrm{~h}$ at $0{ }^{\circ} \mathrm{C}$ and then poured into ice-cooled aqueous $\mathrm{NH}_{4} \mathrm{Cl}$ solution. The layers were separated and the aqueous layer was extracted with ethyl acetate $(2 \times 100 \mathrm{~mL})$. The combined organic extracts were dried over $\mathrm{Na}_{2} \mathrm{SO}_{4}$, filtered and concentrated to afford the corresponding protected amino alcohol as a colorless solid residue, which is used in the next step without further purification.

The Boc $N$-protected amino alcohol, afforded in the previous step, was treated with $52 \mathrm{~mL}$ of $1 \mathrm{M}$ methanolic $\mathrm{HCl}$ at $50{ }^{\circ} \mathrm{C}$ for $3 \mathrm{~h}$. After this time, $\mathrm{MeOH}$ was evaporated under reduced pressure affording a colorless solid residue, which was partitioned between $1 \mathrm{~m} \mathrm{NaOH}$ solution $(90 \mathrm{~mL})$ and $100 \mathrm{~mL}$ of ethyl acetate. The layers were separated and the aqueous layer was extracted with ethyl acetate $(2 \times 80 \mathrm{~mL})$. The combined organic layers were dried over $\mathrm{Na}_{2} \mathrm{SO}_{4}$, filtered and concentrated under reduced pressure. The yellowish-white solid residue was purified by flash chromatography (PET/AcOEt) to afford the corresponding amino alcohol.

\section{(S)-2-Amino-3-methyl-1,1-diphenylbutan-1-ol (53)}

Following general procedure GP2, using 50 (4.00 g, $17.3 \mathrm{mmol}, 1.00$ equiv) and phenyl magnesium bromide in THF ( $1 \mathrm{M}, 86.5 \mathrm{~mL}, 86.5 \mathrm{mmol}, 5.00$ equiv). Purification by flash chromatography (Hexane/EtOAc 10:1) afforded 53 (2.64 g, $10.3 \mathrm{mmol}, 60 \%$ overall yield) as a colorless solid.

[18]D. Uraguchi, S. Sakaki, T. Ooi, J. Am. Chem. Soc. 2007, 129, 12392. 
$\mathrm{R}_{f} 0.1$ (PET/AcOEt 5:1, UV/anisaldehyde). ${ }^{1} \mathrm{H}$ NMR (400 MHz, $\left.\mathrm{CDCl}_{3}\right) \delta 7.65(\mathrm{~d}, J=7.2$ $\mathrm{Hz}, 2 \mathrm{H} ; \mathrm{ArH}), 7.53$ (d, $J=7.0 \mathrm{~Hz}, 2 \mathrm{H} ; \mathrm{ArH}), 7.34$ (t, $J=7.4 \mathrm{~Hz}, 2 \mathrm{H} ; \mathrm{ArH}), 7.30$ (t, $J=6.0$ $\mathrm{Hz}, 2 \mathrm{H}$; $\mathrm{ArH}$ ), 7.20 (m, $J=6.0 \mathrm{~Hz}, 2 \mathrm{H}$; $\mathrm{ArH}$ ), 3.88 (d, $J=2.1 \mathrm{~Hz}, 1 \mathrm{H} ; \mathrm{NH}_{2} \mathrm{CH}$ ), 1.79 (quin$\left.\mathrm{d}, J=6.9,2.1 \mathrm{~Hz}, 1 \mathrm{H} ;\left(\mathrm{CH}_{3}\right)_{2} \mathrm{CH}\right), 0.96\left(\mathrm{~d}, J=7.1 \mathrm{~Hz}, 3 \mathrm{H} ; \mathrm{CH}_{3}\right), 0.92(\mathrm{~d}, J=6.8 \mathrm{~Hz}, 3 \mathrm{H}$; $\left.\mathrm{CH}_{3}\right) .{ }^{13} \mathrm{C}$ NMR $\left(100 \mathrm{MHz}, \mathrm{CDCl}_{3}\right) \delta 148.1,145.0,128.5,128.1,126.7,126.4,126.0,125.6$, 79.8, 60.2, 27.9, 23.1, 16.2. IR v 3087 (w), 3059 (w), 3025 (w), 2960 (m), 2929 (w), 2876 (w), $2361(\mathrm{w}), 2342(\mathrm{w}), 1774(\mathrm{w}), 1652(\mathrm{w}), 1595(\mathrm{~m}), 1491(\mathrm{~m}), 1472(\mathrm{w}), 1448(\mathrm{~m}), 1387$ (w), $1368(\mathrm{w}), 1300(\mathrm{w}), 1252(\mathrm{w}), 1176(\mathrm{~m}), 1141(\mathrm{w}), 1127(\mathrm{w}), 1051(\mathrm{~m}), 1034(\mathrm{w}), 969$ (m), $944(\mathrm{~m}), 904(\mathrm{~m}), 895(\mathrm{~m}), 805(\mathrm{w}), 776(\mathrm{w}), 756(\mathrm{~m}), 745(\mathrm{~s}), 704(\mathrm{~s}), 670(\mathrm{~m}), 640(\mathrm{~m})$, $621(\mathrm{w})$. The characterization data corresponded to the reported values. ${ }^{[18]}$

\section{(S)-2-amino-3-methyl-1,1-di-p-tolylbutan-1-ol (54)}

Following general procedure GP2, using 50 (4.00 g, $17.3 \mathrm{mmol}, 1.00$ equiv) and $p$-tolyl magnesium bromide in THF ( $1 \mathrm{M}, 86.5 \mathrm{~mL}, 86.5 \mathrm{mmol}, 5.00$ equiv). Purification by flash chromatography (Hexane/EtOAc 30:1 to 20:1) afforded 54 (1.54 g, $5.43 \mathrm{mmol}, 31 \%$ overall yield) as a colorless solid.

$\mathrm{R}_{f} 0.1$ (PET/AcOEt 5:1, UV/anisaldehyde). ${ }^{1} \mathrm{H}$ NMR (400 MHz, $\left.\mathrm{CDCl}_{3}\right) \delta 7.50(\mathrm{~d}, J=8.2$ $\mathrm{Hz}, 2 \mathrm{H} ; \operatorname{Ar} H), 7.38$ (d, $J=8.2 \mathrm{~Hz}, 2 \mathrm{H} ; \operatorname{Ar} H), 7.13(\mathrm{~d}, J=8.1 \mathrm{~Hz}, 2 \mathrm{H} ; \operatorname{Ar} H), 7.10$ (d, $J=8.1$ $\mathrm{Hz}, 2 \mathrm{H} ; \mathrm{ArH}$ ), 3.81 (d, $J=2.0 \mathrm{~Hz}, 1 \mathrm{H} ; \mathrm{NH}_{2} \mathrm{CH}$ ), 2.31 (s, $\left.3 \mathrm{H} ; \mathrm{PhCH}_{3}\right), 2.30$ (s, $3 \mathrm{H} ; \mathrm{PhCH}_{3}$ ), 1.79 (quin-d, $\left.J=6.9,2.0 \mathrm{~Hz}, 1 \mathrm{H} ;\left(\mathrm{CH}_{3}\right)_{2} \mathrm{CH}\right), 0.91\left(\mathrm{~d}, J=2.9 \mathrm{~Hz}, 3 \mathrm{H} ; \mathrm{CH}_{3}\right), 0.89$ (d, $J=3.0$ $\left.\mathrm{Hz}, 3 \mathrm{H} ; \mathrm{CH}_{3}\right)$. The characterization data corresponded to the reported values ${ }^{[18]}$

\section{Preparation of amino alcohol hydrochloride 55 and 56}

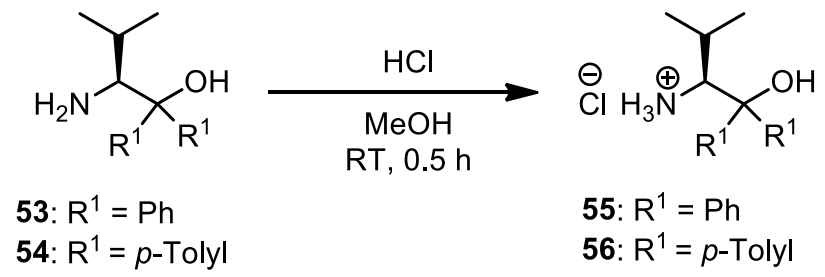

General procedure 3 (GP3): $1 \mathrm{M}$ methanolic $\mathrm{HCl}$ solution $(0.54 \mathrm{M})$ was added to the corresponding amino alcohol (1.0 equiv) and the mixture was stirred at RT. After 15 minutes of stirring, a colorless solid started to form and after the next 5 minutes the mixture solidified completely. The stirring was stopped and the solvents were evaporated under reduced pressure. The amino alcohol hydrochloride was obtained as a pure product and was used in the next step without further purification.

\section{(S)-1-hydroxy-3-methyl-1,1-diphenylbutan-2-aminium chloride (55)}

Following general procedure GP3, using 53 (2.64 g, $10.3 \mathrm{mmol}, 1.00$ equiv) and a $1 \mathrm{M}$ methanolic $\mathrm{HCl}$ solution (19 $\mathrm{mL})$ afforded $\mathbf{5 5}(3.00 \mathrm{~g}, 10.3 \mathrm{mmol}$, quantitative) as a colorless solid which was used without further purification.

\section{(S)-1-hydroxy-3-methyl-1,1-di-p-tolylbutan-2-aminium chloride (56)}

Following general procedure GP3, using 54 (1.54 g, $5.43 \mathrm{mmol}, 1.00$ equiv) and a $1 \mathrm{M}$ methanolic $\mathrm{HCl}$ solution (10 $\mathrm{mL})$ afforded $56(1.54 \mathrm{~g}, 5.43 \mathrm{mmol}$, quantitative) as a colorless solid which was used without further purification. 


\section{Preparation of diamines 215 and 216}

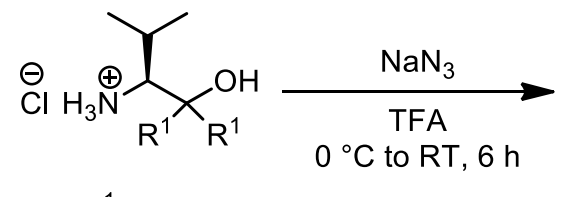

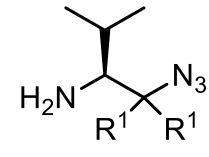

57: $\mathrm{R}^{1}=\mathrm{Ph}$

58: $\mathrm{R}^{1}=p$-Tolyl

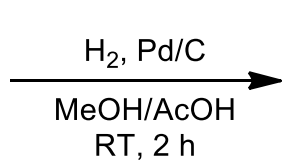

$\overbrace{\mathrm{R}^{1}} x_{\mathrm{R}^{1}}^{\mathrm{NH}_{2}}$

59: $\mathrm{R}^{1}=\mathrm{Ph}, 70 \%$

60: $\mathrm{R}^{1}=p$-Tolyl, $14 \%$

General procedure 4 (GP4): Following the reported procedure, ${ }^{[18]}$ sodium azide (5.0 equiv) was added portionwise carefully to TFA $(0.2 \mathrm{M})$ at $0{ }^{\circ} \mathrm{C}$, followed by portionwise addition of amino alcohol hydrochloride (1.0 equiv). The resulting mixture was stirred for $6 \mathrm{~h}$ at RT. After this time, the mixture was poured into crushed ice with stirring and was neutralized by portionwise addition of $\mathrm{NaOH}$ pellets until $\mathrm{pH}$ reached $11 \approx 12$ (the colour of the suspension was changing upon addition of $\mathrm{NaOH}$ ). The mixture was extracted with ethyl acetate $(3 \times 100 \mathrm{~mL})$. The combined organic layers were washed with brine $(2 \times 100 \mathrm{~mL})$, dried over $\mathrm{Na}_{2} \mathrm{SO}_{4}$, filtered and concentrated under reduced pressure to afford the corresponding amino azide product. The obtained residue was used in the next step without further purification.

Crude amino azide (1.00 equiv) was dissolved in $\mathrm{MeOH}(0.2 \mathrm{M})$ containing glacial $\mathrm{AcOH}$ (1.0 equiv). The mixture was cooled to $0{ }^{\circ} \mathrm{C}$ under nitrogen atmosphere and $10 \% \mathrm{Pd} / \mathrm{C}(0.05$ equiv) was added. The atmosphere was replaced with hydrogen and the final mixture was stirred at room temperature until completed conversion. $\mathrm{Pd} / \mathrm{C}$ was then removed by filtration (syringe filter) and the filtrate was concentrated under reduced pressure. The obtained residue was neutralized with $1 \mathrm{M}$ aq. $\mathrm{NaOH}$ and extracted with ethyl acetate $(3 \times 80 \mathrm{~mL})$. The combined organic extracts were dried over $\mathrm{Na}_{2} \mathrm{SO}_{4}$, filtered and the solvent was evaporated under reduced pressure. Purification by column chromatography $(\mathrm{AcOEt} / \mathrm{MeOH})$ afforded the diamine product.

\section{(2S)-3-methyl-1,1-diphenylbutane-1,2-diamine (59)}

Following general procedure GP4, the reaction of 55 (2.95 g, $10.1 \mathrm{mmol}, 1.00$ equiv), $\mathrm{NaN}_{3}(3.32 \mathrm{~g}, 50.5 \mathrm{mmol}, 5.00$ equiv) and TFA (50.5 mL) afforded 57 (2.80 g, $10.1 \mathrm{mmol}$, quantitative) used in the next step without further purification.

Crude amino azide 57 (2.80 g, $10.1 \mathrm{mmol}, 1.00$ equiv) was dissolved in $\mathrm{MeOH}(50.5 \mathrm{~mL})$ containing glacial AcOH $(0.580 \mathrm{~mL}, 10.1 \mathrm{mmol}, 1.00$ equiv). After addition of $10 \% \mathrm{Pd} / \mathrm{C}$ ( $1.01 \mathrm{~g}, 0.505 \mathrm{mmol}, 0.0500$ equiv) the reaction was completed at RT after $2 \mathrm{~h}$. Purification by flash chromatography (AcOEt/MeOH 30:1) afforded the pure product 59 (1.81 g, 7.11 mmol, $70 \%$ overall yield) as a colorless solid.

$\mathrm{R}_{f} 0.1$ (AcOEt/MeOH 40:1, UV/anisaldehyde). ${ }^{1} \mathrm{H}$ NMR $\left(400 \mathrm{MHz}, \mathrm{CDCl}_{3}\right) \delta 7.48(\mathrm{~d}, J=$ $7.4 \mathrm{~Hz}, 2 \mathrm{H}$; $\operatorname{ArH}), 7.45$ (d, J=7.4 Hz, $2 \mathrm{H}$; $\operatorname{ArH}), 7.30$ (t, $J=7.4 \mathrm{~Hz}, 4 \mathrm{H}$; ArH), 7.19 (m, 2 $\mathrm{H} ; \mathrm{ArH}), 3.70$ (d, $J=1.6 \mathrm{~Hz}, 1 \mathrm{H} ; \mathrm{NH}_{2} \mathrm{CH}$ ), 1.89 (quin-d, $J=6.9,2.1 \mathrm{~Hz}, 1 \mathrm{H} ;\left(\mathrm{CH}_{3}\right)_{2} \mathrm{CH}$ ), 1.75 (br s, $4 \mathrm{H}$; $\left.\left(\mathrm{NH}_{2}\right)_{2}\right), 1.01\left(\mathrm{~d}, J=6.9 \mathrm{~Hz}, 3 \mathrm{H} ; \mathrm{CH}_{3}\right), 0.74$ (d, $J=6.8 \mathrm{~Hz}, 3 \mathrm{H}$; CH $\mathrm{CH}_{3}$ ). IR v 3058 (w), 3022 (w), 2958 (m), $2871(\mathrm{w}), 2364(\mathrm{w}), 2339(\mathrm{w}), 1599(\mathrm{w}), 1492(\mathrm{~m}), 1447(\mathrm{~m})$, $1365(\mathrm{w}), 1174(\mathrm{w}), 1034(\mathrm{w}), 912(\mathrm{~m}), 870$ (w), $768(\mathrm{w}), 741(\mathrm{~m}), 704(\mathrm{~s}), 670(\mathrm{~m}), 652(\mathrm{~m})$, $632(\mathrm{~m}), 622(\mathrm{~m}), 600(\mathrm{w})$. The characterization data corresponded to the reported values. ${ }^{[18]}$

(2S)-3-methyl-1,1-di-p-tolylbutane-1,2-diamine (60) 
Following general procedure GP4, the reaction of $\mathbf{5 6}(1.50 \mathrm{~g}, 4.69 \mathrm{mmol}, 1.00$ equiv), $\mathrm{NaN}_{3}(1.52 \mathrm{~g}, 23.5 \mathrm{mmol}, 5.00$ equiv) and TFA (23.5 mL) afforded 58 (1.50 g, $4.69 \mathrm{mmol}$, quantitative) used in the next step without further purification.

Crude amino azide 58 ( $1.50 \mathrm{~g}, 4.69 \mathrm{mmol}, 1.00$ equiv) was dissolved in $\mathrm{MeOH}(23.5 \mathrm{~mL})$ containing glacial AcOH $(0.268 \mathrm{~mL}, 4.69 \mathrm{mmol}, 1.00$ equiv). After addition of $10 \% \mathrm{Pd} / \mathrm{C}$ ( $0.50 \mathrm{~g}, 0.23 \mathrm{mmol}, 0.050$ equiv) the reaction was completed at RT after $2 \mathrm{~h}$. Purification by flash chromatography (AcOEt/MeOH 30:1) afforded the pure product 60 (184 mg, 0.652 mmol, $14 \%$ overall yield) as a colorless solid.

$\mathrm{R}_{f} 0.1$ (AcOEt/MeOH 40:1, UV/anisaldehyde). ${ }^{1} \mathrm{H}$ NMR (400 MHz, $\left.\mathrm{CDCl}_{3}\right) \delta 7.36(\mathrm{~d}, J=$ $8.2 \mathrm{~Hz}, 2 \mathrm{H} ; \operatorname{Ar} H), 7.33(\mathrm{~d}, J=8.2 \mathrm{~Hz}, 2 \mathrm{H} ; \operatorname{Ar} H), 7.09$ (m, $4 \mathrm{H} ; \operatorname{Ar} H), 3.60(\mathrm{~d}, J=3.9 \mathrm{~Hz}, 1$ $\mathrm{H} ; \mathrm{NH}_{2} \mathrm{CH}$ ), 2.67 (br s, $4 \mathrm{H}$; $\left.\left(\mathrm{NH}_{2}\right)_{2}\right), 2.33$ (s, $\left.3 \mathrm{H} ; \mathrm{PhCH}_{3}\right), 2.31$ (s, $\left.3 \mathrm{H} ; \mathrm{PhCH}_{3}\right), 1.83$ (br quin-d, $\left.1 \mathrm{H} ;\left(\mathrm{CH}_{3}\right)_{2} \mathrm{CH}\right), 1.01\left(\mathrm{~d}, J=6.9 \mathrm{~Hz}, 3 \mathrm{H} ; \mathrm{CH}_{3}\right), 0.74\left(\mathrm{~d}, J=6.8 \mathrm{~Hz}, 3 \mathrm{H} ; \mathrm{CH}_{3}\right)$. The characterization data corresponded to the reported values. ${ }^{[18]}$

\section{Preparation of tetraaminophosphonium salts 11a and 11b}

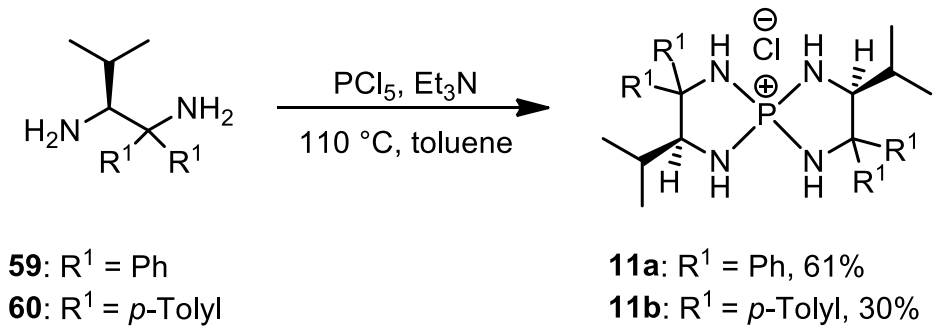

General procedure 5 (GP5): Following the reported procedure, ${ }^{[18]}$ a solution of $\mathrm{PCl}_{5}(0.5$ equiv) in toluene $(0.2 \mathrm{M})$ was added to a solution of diamine (1.0 equiv) and $\mathrm{Et}_{3} \mathrm{~N}$ (2.5 equiv) in toluene $(0.4 \mathrm{M})$ at room temperature. The final mixture was stirred at $110{ }^{\circ} \mathrm{C}$ for $3 \mathrm{~h}$. After this time, the volatiles were evaporated and the final residue was dissolved into $\mathrm{CHCl}_{3}$ and washed with $1 \mathrm{~N} \mathrm{HCl}$ solution. The organic extracts were recollected, dried over $\mathrm{Na}_{2} \mathrm{SO}_{4}$, filtered and evaporated under reduced pressure. The residual yellow oil was purified by flash chromatography (AcOEt/MeOH) to afford the pure product as a mixture of diastereomers, which were separated by recrystallization from acetone/hexane solvent system at $-15{ }^{\circ} \mathrm{C}$.

\section{(3S,8S)-3,8-Diisopropyl-2,2,7,7-tetraphenyl-1,4,6,9-tetraaza-5-phosphaspiro [4.4]nonan-5-ium chloride (11a)}

Following general procedure GP5, the reaction of $\mathbf{5 9}(1.70 \mathrm{~g}, 6.68 \mathrm{mmol}, 1.00$ equiv), $\mathrm{Et}_{3} \mathrm{~N}$ (2.32 mL, $16.7 \mathrm{mmol}, 2.50$ equiv) in toluene $(16.7 \mathrm{~mL})$ and $\mathrm{PCl}_{5}(690 \mathrm{mg}, 3.34 \mathrm{mmol}$, 0.500 equiv) in toluene (16.7 $\mathrm{mL})$ afforded $11 \mathrm{a}(2.33 \mathrm{~g}, 4.08 \mathrm{mmol}, 61 \%, M S: P S=3: 1)$ as a mixture of diastereomers after purification by flash chromatography (AcOEt/MeOH 30:1). The mixture of diastereomers was dissolved in acetone at room temperature. The solution was then cooled at $-15^{\circ} \mathrm{C}$ and hexane was dropwise added until formation of precipitate. The obtained precipitate was filtered and dried under vacuum to obtain $(M, S)$-11a in an essentially pure form as a pink solid.

(M,S)-11a pink solid; $\mathrm{R}_{f} 0.1$ (AcOEt/MeOH 40:1, UV/anisaldehyde). ${ }^{1} \mathrm{H}$ NMR (400 MHz, MeOD) $\delta 7.48$ (d, $J=7.9 \mathrm{~Hz}, 4 \mathrm{H}$; ArH) 7.42 (t, $J=7.4 \mathrm{~Hz}, 4 \mathrm{H} ; \operatorname{Ar} H), 7.33$ (m, $6 \mathrm{H}$; ArH), $7.24(\mathrm{t}, J=6.7 \mathrm{~Hz}, 4 \mathrm{H} ; \mathrm{ArH}), 7.19(\mathrm{tt}, J=5.7,1.5 \mathrm{~Hz}, 2 \mathrm{H} ; \mathrm{ArH}), 6.55\left(\mathrm{~d}, J_{\mathrm{P}-\mathrm{H}}=17.6 \mathrm{~Hz}, 2\right.$ $\mathrm{H} ; \mathrm{PN} H), 6.36\left(\mathrm{~d}, J_{\mathrm{P}-\mathrm{H}}=20.3 \mathrm{~Hz}, 2 \mathrm{H} ; \mathrm{PN} H\right), 4.34\left(\mathrm{dd}, J_{\mathrm{P}-\mathrm{H}}=24.1 \mathrm{~Hz}, J_{\mathrm{H}-\mathrm{H}}=3.3 \mathrm{~Hz}, 2 \mathrm{H}\right.$; 
$\mathrm{PNH}_{2}$ ), 1.61 (br quin-d, $2 \mathrm{H}$; $\left.\left(\mathrm{CH}_{3}\right)_{2} \mathrm{CH}\right), 0.88$ (d, $\left.J=6.5 \mathrm{~Hz}, 6 \mathrm{H} ;\left(\mathrm{CH}_{3}\right)_{2}\right), 0.77$ (d, $J=6.7$ $\left.\mathrm{Hz}, 6 \mathrm{H} ;\left(\mathrm{CH}_{3}\right)_{2}\right) .{ }^{13} \mathrm{C}$ NMR $(101 \mathrm{MHz}, \mathrm{MeOD}) \delta 148.4,142.2\left(\mathrm{~d}, J_{\mathrm{P}-\mathrm{C}}=12 \mathrm{~Hz}\right), 129.7,128.9$, $128.6,128.3,128.2,127.6,71.4\left(\mathrm{~d}, J_{\mathrm{P}-\mathrm{C}}=13 \mathrm{~Hz}\right), 65.5\left(\mathrm{~d}, J_{\mathrm{P}-\mathrm{C}}=8 \mathrm{~Hz}\right), 30.9,21.7,17.5 .{ }^{31} \mathrm{P}$

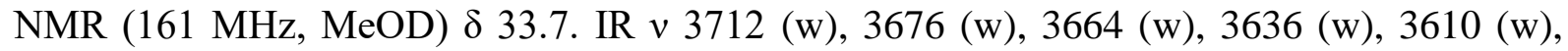
3567 (w), 3520 (w), 3062 (w), 3036 (w), 2960 (w), 2872 (w), 2360 (w), $2151(w), 2073$ (w), 1740 (w), 1601 (w), 1495 (w), 1449 (w), 1322 (w), 1290 (w), $1241(\mathrm{w}), 1194(\mathrm{w}), 1169$ (w), 1126 (w), 1094 (w), 1057 (w), $1000(\mathrm{w}), 975$ (w), 882 (w), 837 (w), 784 (w), 754 (w), 704 $(\mathrm{m}), 670(\mathrm{w}), 659(\mathrm{~m}), 645(\mathrm{~s}), 633(\mathrm{~s}), 622(\mathrm{~m})$. ). The characterization data corresponded to the reported values. ${ }^{[18]}$

\section{(3S,8S)-3,8-Diisopropyl-2,2,7,7-tetra-p-tolyl-1,4,6,9-tetraaza-5-phosphaspiro [4.4]nonan-5-ium chloride (11b)}

Following general procedure GP5, the reaction of $\mathbf{6 0}$ (183 $\mathrm{mg}, 0.648 \mathrm{mmol}, 1.00$ equiv), $\mathrm{Et}_{3} \mathrm{~N}\left(0.230 \mathrm{~mL}, 1.62 \mathrm{mmol}, 2.50\right.$ equiv) in toluene $(1.62 \mathrm{~mL})$ and $\mathrm{PCl}_{5}(67.0 \mathrm{mg}, 0.324$ mmol, 0.500 equiv) in toluene $(1.62 \mathrm{~mL})$ afforded $11 \mathrm{~b}(122 \mathrm{mg}, 0.194 \mathrm{mmol}, 30 \%, M S: P S=$ 2.3:1) as a colorless solid and as a mixture of diastereomers after purification by flash chromatography (AcOEt/MeOH 30:1).

For mixture of $(\boldsymbol{M}, \boldsymbol{S})-\mathbf{1 1 b}$ and $(\boldsymbol{P}, \boldsymbol{S})-\mathbf{1 1 b}$ white solid. $\mathrm{R}_{f} 0.1$ (AcOEt/MeOH 40:1, UV/anisaldehyde). ${ }^{1} \mathrm{H}$ NMR (400 MHz, MeOD) (diastereomers $\left.M S: P S-2.3: 1\right) \delta 7.43(\mathrm{~d}, J=$ $8.3 \mathrm{~Hz}, 1.7 \mathrm{H}$; ArH-PS), 7.34 (d, $J=8.3 \mathrm{~Hz}, 4 \mathrm{H} ; \operatorname{Ar} H), 7.27$ (d, $J=8.2 \mathrm{~Hz}, 1.7 \mathrm{H}$; ArH-PS), 7.22 (d, $7 \mathrm{H}, J=8.4 \mathrm{~Hz}, 4 \mathrm{H}$; ArH), 7.19 (s overlapping, $1 \mathrm{H}$; ArH-PS), 7.15 (d, $J=8.3 \mathrm{~Hz}, 4$ $\mathrm{H} ; \operatorname{Ar} H), 7.11(\mathrm{~d}, J=8.1 \mathrm{~Hz}, 1.7 \mathrm{H}$; $\operatorname{Ar} H-P S), 7.03(\mathrm{~d}, J=8.1 \mathrm{~Hz}, 4 \mathrm{H} ; \mathrm{Ar} H), 4.24\left(\mathrm{dd}, J_{\mathrm{P}-\mathrm{H}}=\right.$ $\left.24.0, J_{\mathrm{H}-\mathrm{H}}=3.5 \mathrm{~Hz}, 2 \mathrm{H} ; \mathrm{PNH}_{2}\right), 3.95\left(\mathrm{dd}, J_{\mathrm{P}-\mathrm{H}}=23.0, J_{\mathrm{H}-\mathrm{H}}=5.6 \mathrm{~Hz}, 0.86 \mathrm{H} ; \mathrm{PNH}_{2}-P S\right), 2.35$ (s, $\left.6 \mathrm{H} ; \mathrm{Ph}\left(\mathrm{CH}_{3}\right)_{2}\right), 2.32$ (s, $\left.2.6 \mathrm{H} ; \mathrm{Ph}\left(\mathrm{CH}_{3}\right)_{2}-P S\right), 2.30$ (s, $\left.2.6 \mathrm{H} ; \mathrm{Ph}\left(\mathrm{CH}_{3}\right)_{2}-P S\right), 2.25$ (s, $6 \mathrm{H}$; $\mathrm{Ph}\left(\mathrm{CH}_{3}\right)_{2}$ ), 1.68 (br quin-d, $0.86 \mathrm{H}$; $\left.\left(\mathrm{CH}_{3}\right)_{2} \mathrm{CH}\right), 1.59$ (br quin-d, $\left.2 \mathrm{H} ;\left(\mathrm{CH}_{3}\right)_{2} \mathrm{CH}\right), 0.89$ (s overlaping, $2.6 \mathrm{H}$; $\left.\left(\mathrm{CH}_{3}\right)_{2}-P S\right), 0.86\left(\mathrm{~d}, J=6.6 \mathrm{~Hz}, 6 \mathrm{H}\right.$; $\left.\left(\mathrm{CH}_{3}\right)_{2}\right), 0.76$ (s overlaping, $2.6 \mathrm{H}$; $\left.\left(\mathrm{CH}_{3}\right)_{2}-\mathrm{PS}\right), 0.74\left(\mathrm{~d}, J=6.9 \mathrm{~Hz}, 6 \mathrm{H}\right.$; $\left.\left(\mathrm{CH}_{3}\right)_{2}\right) .{ }^{13} \mathrm{C} \mathrm{NMR}(101 \mathrm{MHz}, \mathrm{MeOD}) \delta 145.7,145.4$, $139.4\left(\mathrm{~d}, J_{\mathrm{P}-\mathrm{C}}=3.1 \mathrm{~Hz}\right), 139.3,138.3\left(\mathrm{~d}, J_{\mathrm{P}-\mathrm{C}}=3.6 \mathrm{~Hz}\right), 138.2,138.0,130.6,130.1,130.1$, $129.5,129.4,128.9,128.2,127.7,127.5,71.1\left(\mathrm{~d}, J_{\mathrm{P}-\mathrm{C}}=12 \mathrm{~Hz}\right), 70.6\left(\mathrm{~d}, J_{\mathrm{P}-\mathrm{C}}=14 \mathrm{~Hz}\right), 66.5$ $\left(\mathrm{d}, J_{\mathrm{P}-\mathrm{C}}=7 \mathrm{~Hz}\right), 65.6\left(\mathrm{~d}, J_{\mathrm{P}-\mathrm{C}}=8 \mathrm{~Hz}\right), 30.8,30.5,21.7,21.4,18.7,17.6 .{ }^{31} \mathrm{P}$ NMR $(161 \mathrm{MHz}$, MeOD) $\delta$ 33.7, 32.8. IR v 3060 (w), $3057(w), 3028(w), 3026(w), 2962(w), 2925(w), 2875$ (w), $2831(\mathrm{w}), 2360(\mathrm{w}), 2341(\mathrm{w}), 2310(\mathrm{w}), 2292(\mathrm{w}), 2239$ (w), $2188(\mathrm{w}), 2158(\mathrm{w}), 2075$ (w), 1720 (w), 1649 (m), 1578 (w), 1513 (m), $1470(\mathrm{w}), 1450$ (w), 1409 (m), 1388 (m), 1325 (w), $1286(\mathrm{w}), 1188(\mathrm{~s}), 1168(\mathrm{~s}), 1125(\mathrm{~m}), 1122(\mathrm{~m}), 1068(\mathrm{~m}), 1021(\mathrm{~m}), 977(\mathrm{~m}), 905(\mathrm{w})$, $812(\mathrm{~s}), 779(\mathrm{~m}), 725(\mathrm{~m}), 691(\mathrm{~m}), 674(\mathrm{~m}), 652(\mathrm{~s}), 643(\mathrm{~s})$. ). The characterization data corresponded to the reported values. ${ }^{[18]}$

\section{Alkynylation Reactions}

General procedure 6 (GP6): A solution of substrate (1.0 equiv) and alkynyl benziodoxolone reagent (1.3 equiv) in dried THF $(60 \mathrm{mM})$ was stirred at $-78{ }^{\circ} \mathrm{C}$ for $5 \mathrm{~min}$ under nitrogen. After this period of time, TBAF ( $1 \mathrm{M}$ in THF, 1.3 equiv) was added and the mixture was vigorously stirred at $-78{ }^{\circ} \mathrm{C}$. The reaction was monitored by TLC analysis (PET/EtOAc, 4:1, UV and $p$-anisaldehyde) and was complete at $-78{ }^{\circ} \mathrm{C}$ in the indicated time, or was slowly let to warm up to $10{ }^{\circ} \mathrm{C}$ during the indicated time. The reaction mixture was quenched with deactivated silica gel and the solvent was evaporated under reduced pressure. 
The product was purified via flash chromatography $\left(\mathrm{SiO}_{2}, \mathrm{Hexane} / \mathrm{EtOAc}\right)$ with the indicated solvent ratio.

General procedure 7 (GP7): A solution of saturated base solution $(0.2 \mathrm{M})$ was added to a solution of phase-transfer catalyst (10 mol\%) and alkynyl benziodoxolone reagent (1.3 equiv) in dry toluene $(50 \mathrm{mM})$. The mixture was stirred at $0{ }^{\circ} \mathrm{C}$ for $5 \mathrm{~min}$ under nitrogen. After this period of time, the substrate (1.0 equiv) was added and the biphasic mixture was vigorously stirred at $0{ }^{\circ} \mathrm{C}$. The reaction was monitored by TLC analysis (PET/EtOAc 4:1, UV and $p$ anisaldehyde). After the indicated time, the reaction mixture was quenched with water and the aqueous layer was extracted with $\mathrm{CH}_{2} \mathrm{Cl}_{2}$. The combined organic layers were recollected, dried over $\mathrm{MgSO}_{4}$, filtered and concentrated under reduced pressure. The crude product was purified via silica gel flash chromatography $\left(\mathrm{SiO}_{2}\right.$, Hexane/EtOAc) with the indicated solvent ratio.

General procedure 8 (GP8): A saturated base solution $(0.2 \mathrm{M})$ was added to a solution of phase-transfer catalyst ( $3 \mathrm{~mol} \%)$ and alkynyl benziodoxolone reagent (1.3 equiv) in xylene $(50 \mathrm{mM})$. The mixture was stirred at $0{ }^{\circ} \mathrm{C}$ for $5 \mathrm{~min}$ under nitrogen. After this period of time, the substrate (1.0 equiv) was added and the biphasic mixture was vigorously stirred at $0{ }^{\circ} \mathrm{C}$. The reaction was monitored by TLC analysis (PET/EtOAc 4:1, UV and $p$-anisaldehyde) and was complete at $0{ }^{\circ} \mathrm{C}$ in the indicated time, or was slowly let to warm up to $10{ }^{\circ} \mathrm{C}$ during the indicated time. The reaction mixture was quenched with water and the aqueous layer extracted with $\mathrm{CH}_{2} \mathrm{Cl}_{2}$. The combined organic layers were recollected, dried over $\mathrm{MgSO}_{4}$, filtered and concentrated under reduced pressure. The crude product was purified via silica gel flash chromatography $\left(\mathrm{SiO}_{2}, \mathrm{Hexane} / \mathrm{EtOAc}\right)$ with the indicated solvent ratio.

\section{Methyl 2-ethynyl-1-oxo-2,3-dihydro-1H-indene-2-carboxylate (3a)}

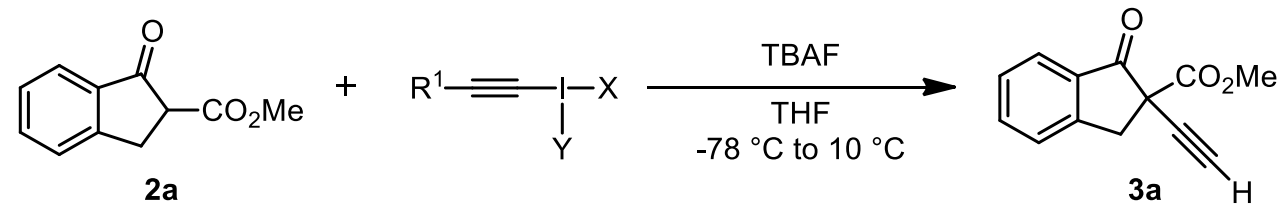

Following general procedure GP6, on a $0.40 \mathrm{mmol}$ scale using 2a $(76 \mathrm{mg}, 0.40 \mathrm{mmol}, 1.0$ equiv) and TMS-EBX (1a) (179 $\mathrm{mg}, 0.520 \mathrm{mmol}, 1.3$ equiv). The reaction was quenched after $1.5 \mathrm{~h}$ at $-78{ }^{\circ} \mathrm{C}$. Purification by flash chromatography (Hexane/EtOAc 10:1) afforded 3a (83 $\mathrm{mg}, 0.39 \mathrm{mmol}, 98 \%$ ) as yellow solid.

Following general procedure GP6, on a $0.40 \mathrm{mmol}$ scale using $\mathbf{2 a}(76 \mathrm{mg}, 0.40 \mathrm{mmol}, 1.0$ equiv) and TIPS-EBX (1b) (220 mg, $0.520 \mathrm{mmol}, 1.3$ equiv). The reaction was quenched after $14.5 \mathrm{~h}$ after the temperature had reached $10{ }^{\circ} \mathrm{C}$. Purification by flash chromatography (Hexane/EtOAc 10:1) afforded $\mathbf{3 a}(68.8 \mathrm{mg}, 0.321 \mathrm{mmol}, 80 \%)$ as yellow solid.

Following general procedure GP6, on a $0.20 \mathrm{mmol}$ scale using 2a (40 mg, $0.20 \mathrm{mmol}, 1.0$ equiv) and trimethylsilylethynyl(phenyl)iodonium triflate (4) $(123 \mathrm{mg}, 0.273 \mathrm{mmol}, 1.3$ equiv). The reaction was quenched after $20.5 \mathrm{~h}$ at $-78{ }^{\circ} \mathrm{C}$. Purification by flash chromatography (Hexane/EtOAc 20:1) afforded 3a (45 mg, $0.21 \mathrm{mmol}, 89 \%$ ) as yellow solid. 
$\mathrm{R}_{f} 0.33$ (PET/EtOAc 4:1, UV/Anisaldehyde). Mp 96-98 ${ }^{\circ} \mathrm{C} .{ }^{1} \mathrm{H}$ NMR (400 MHz, $\mathrm{CDCl}_{3}$ ) $\delta 7.83(\mathrm{~d}, J=7.7 \mathrm{~Hz}, 1 \mathrm{H} ; \operatorname{Ar} H), 7.67(\mathrm{t}, J=7.5 \mathrm{~Hz}, 1 \mathrm{H}$; $\operatorname{Ar} H), 7.49(\mathrm{~d}, J=7.7 \mathrm{~Hz}, 1 \mathrm{H}$; $\operatorname{ArH}), 7.43(\mathrm{t}, J=7.5 \mathrm{~Hz}, 1 \mathrm{H}$; $\mathrm{ArH}), 3.94\left(\mathrm{~d}, J=17.1 \mathrm{~Hz}, 1 \mathrm{H} ; \mathrm{CH}_{2}\right), 3.80$ (s, 3H; CH$)_{3}, 3.52$ $\left(\mathrm{d}, J=17.1 \mathrm{~Hz}, 1 \mathrm{H} ; \mathrm{CH}_{2}\right), 2.42(\mathrm{~s}, 1 \mathrm{H} ; \mathrm{C} \equiv \mathrm{CH}) .{ }^{13} \mathrm{C} \mathrm{NMR}\left(101 \mathrm{MHz}, \mathrm{CDCl}_{3}\right) \delta 195.5,167.9$, 151.9, 135.9, 132.7, 128.0, 126.2, 125.4, 79.6, 72.2, 54.9, 53.5, 40.2. IR v $3283(\mathrm{w}), 2976(\mathrm{w})$, 2937 (w), 2877 (w), 1730 (s), 1608 (w), 1462 (w), $1436(w), 1368(w), 1262(w), 1214(w)$, $1152(\mathrm{~m}), 1066(\mathrm{w}), 958(\mathrm{w}), 913(\mathrm{w}), 847(\mathrm{w}), 802(\mathrm{w}), 740(\mathrm{w}), 687(\mathrm{w}), 639(\mathrm{w})$. HRMS(ESI) calcd for $\mathrm{C}_{13} \mathrm{H}_{10} \mathrm{O}_{3} \mathrm{Na}^{+}(\mathrm{M}+\mathrm{Na}) 237.0528$, found 237.0524 .

\section{Tert-Butyl 2-ethynyl-1-oxo-2,3-dihydro-1H-indene-2-carboxylate (3b)}

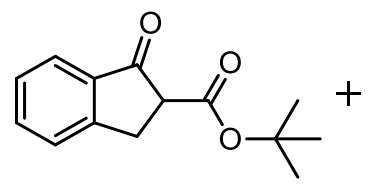

$2 \mathbf{b}$

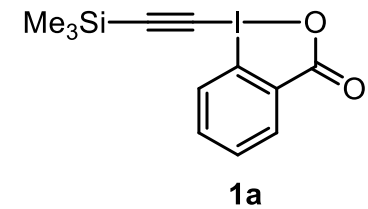

$1 \mathrm{a}$

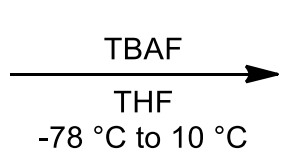

$-78^{\circ} \mathrm{C}$ to $10^{\circ} \mathrm{C}$

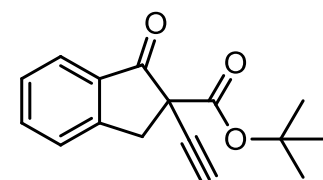

$3 \mathbf{b}$

Following general procedure GP6, on a $0.40 \mathrm{mmol}$ scale using $\mathbf{2 b}$ (93 $\mathrm{mg}, 0.40 \mathrm{mmol}, 1.0$ equiv) and TMS-EBX (1a) (179 mg, $0.520 \mathrm{mmol}, 1.3$ equiv). The reaction was finished after $3 \mathrm{~h}$ at $-78{ }^{\circ} \mathrm{C}$. Purification by flash chromatography (Hexane/EtOAc 20:1) afforded 3b (95.8 $\mathrm{mg}, 0.374 \mathrm{mmol}, 94 \%$ ) as colorless solid.

Reagents 1b-f, 5 and $\mathbf{6}$ were tested following general procedure GP6 on a $0.08 \mathrm{mmol}$ scale using $\mathbf{2 b}$ (1.0 equiv) and EBX reagents (1.3 equiv). The results are given in Table 1.

Following general procedure GP7, on a $0.20 \mathrm{mmol}$ scale using $\mathbf{2 b}$ (46 mg, $0.20 \mathrm{mmol}, 1.0$ equiv), TMS-EBX (1a) ( $89.5 \mathrm{mg}, 0.260 \mathrm{mmol}, 1.30$ equiv), a sat. KF solution ( $33 \% \mathrm{w} / \mathrm{w}, 1.0$ $\mathrm{mL})$ and phosphonium catalyst $11 \mathrm{a}(12 \mathrm{mg}, 0.020 \mathrm{mmol}, 0.1$ equiv), the reaction was finished after $6 \mathrm{~h}$ at $0{ }^{\circ} \mathrm{C}$. The crude product was purified via silica gel flash chromatography (Hexane/EtOAc 15:1) to afford $\mathbf{3 b}(40.0 \mathrm{mg}, 0.156 \mathrm{mmol}, 78 \%)$ as a colorless solid. HPLC analysis with a Daicel Chiralpack IC column; $20{ }^{\circ} \mathrm{C}$; 0 to $32 \mathrm{~min}$; flow rate $1.0 \mathrm{~mL} / \mathrm{min}$; [ ${ }^{i} \mathrm{PrOH} / \mathrm{h}$ exane, 0 to $5 \mathrm{~min} 5 / 95,5$ to $25 \mathrm{~min} 5 / 95$ to 30/70, $25 \mathrm{~min}$ to $27 \mathrm{~min} 30 / 70$ to 5/95, 27 $\min$ to $32 \mathrm{~min} 5 / 95]$; $\tau_{\text {major }}=18.3 \mathrm{~min}, \boldsymbol{\tau}_{\operatorname{minor}}=16.4 \mathrm{~min}$, gave $49 \%$ ee for $\mathbf{3 b}$. Using the same procedure but with catalyst $\mathbf{1 1 b}, \mathbf{3 b}$ was obtained in $90 \%$ yield and $51 \%$ ee.

Following general procedure GP8, on a $0.20 \mathrm{mmol}$ scale using $\mathbf{3 a}$ (46 mg, $0.20 \mathrm{mmol}, 1.0$ equiv), TMS-EBX (1a) ( $89.5 \mathrm{mg}, 0.260 \mathrm{mmol}, 1.30$ equiv), a sat. KF solution ( $33 \% \mathrm{w} / \mathrm{w}, 1.0$ $\mathrm{mL})$ and Maruoka's catalyst $12(4.49 \mathrm{mg}, 6.00 \mu \mathrm{mol}, 0.0300$ equiv), the reaction was finished after $4 \mathrm{~h}$ at $0{ }^{\circ} \mathrm{C}$. The crude product was purified via silica gel flash chromatography (Hexane/EtOAc 20:1) to afford $\mathbf{3 b}(35.9 \mathrm{mg}, 0.140 \mathrm{mmol}, 70 \%)$ as a colorless solid. HPLC analysis with a Daicel Chiralpack IC column; $20{ }^{\circ} \mathrm{C}$; 0 to $32 \mathrm{~min}$; flow rate $1.0 \mathrm{~mL} / \mathrm{min}$; [ ${ }^{i} \mathrm{PrOH} /$ hexane, 0 to $5 \mathrm{~min} 5 / 95,5$ to $25 \mathrm{~min} 5 / 95$ to 30/70, $25 \mathrm{~min}$ to $27 \mathrm{~min} 30 / 70$ to 5/95, 27 $\min$ to $32 \mathrm{~min} 5 / 95] ; \tau_{\text {major }}=18.3 \mathrm{~min}, \tau_{\text {minor }}=16.4 \mathrm{~min}$, gave $65 \%$ ee for $\mathbf{3 b}$. Optical rotation: $[\alpha]_{\mathrm{D}}^{25}=+17.7\left(\mathrm{c}=0.083, \mathrm{CH}_{2} \mathrm{Cl}_{2}\right)$.

$\mathrm{R}_{f} 0.74$ (PET/EtOAc 4:1, UV/Anisaldehyde). Mp 94-97 ${ }^{\circ} \mathrm{C} .{ }^{1} \mathrm{H}$ NMR (400 MHz, $\left.\mathrm{CDCl}_{3}\right) \delta$ $7.83(\mathrm{~d}, J=7.7 \mathrm{~Hz}, 1 \mathrm{H} ; \operatorname{Ar} H), 7.65$ (t, $J=7.5 \mathrm{~Hz}, 1 \mathrm{H} ; \operatorname{Ar} H), 7.49$ (d, $J=7.7 \mathrm{~Hz}, 1 \mathrm{H} ; \operatorname{Ar} H)$, $7.43(\mathrm{t}, J=7.5 \mathrm{~Hz}, 1 \mathrm{H} ; \mathrm{Ar} H), 3.84\left(\mathrm{~d}, J=17.1 \mathrm{~Hz}, 1 \mathrm{H} ; \mathrm{CH}_{2}\right), 3.50(\mathrm{~d}, J=17.1 \mathrm{~Hz}, 1 \mathrm{H}$; 
$\left.\mathrm{CH}_{2}\right), 2.39$ (s, $\left.1 \mathrm{H} ; \mathrm{C} \equiv \mathrm{CH}\right), 1.43\left(\mathrm{~s}, 9 \mathrm{H} ; \mathrm{OC}\left(\mathrm{CH}_{3}\right)_{3}\right) .{ }^{13} \mathrm{C} \mathrm{NMR}\left(101 \mathrm{MHz}, \mathrm{CDCl}_{3}\right) \delta 196.5$, 166.6, 152.2, 135.7, 133.5, 128.1, 126.3, 125.6, 83.5, 80.3, 72.1, 56.2, 40.6, 27.6. IR v 3308 (w), $2981(\mathrm{w}), 2942$ (w), 2866 (w), 2362 (w), 2255 (w), 1723 (m), 1609 (w), 1466 (w), 1372 (w), $1287(\mathrm{w}), 1256(\mathrm{w}), 1153(\mathrm{w}), 907$ (s), 806 (w), 733 (s), 651 (m). HRMS(ESI) calcd for $\mathrm{C}_{16} \mathrm{H}_{16} \mathrm{O}_{3} \mathrm{Na}^{+}(\mathrm{M}+\mathrm{Na})$ 279.0997, found 279.1006. (3b')

Tert-Butyl 1-oxo-2-((triisopropylsilyl)ethynyl)-2,3-dihydro-1 $H$-indene-2-carboxylate

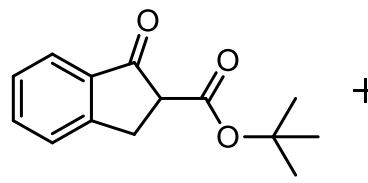

$2 \mathbf{b}$<smiles>O=C1OI(C#C[Se]C(F)(F)F)c2ccccc21</smiles>

$1 \mathrm{~b}$

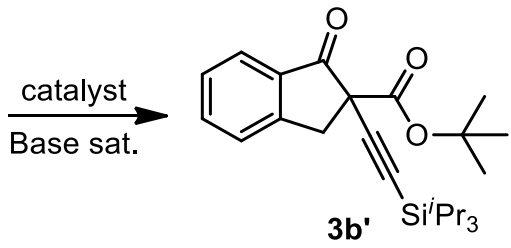

3b' $\mathrm{Si}^{\mathrm{i}} \mathrm{Pr}_{3}$

Following general procedure GP8, on a $0.20 \mathrm{mmol}$ scale using $\mathbf{2 b}$ (46 $\mathrm{mg}, 0.20 \mathrm{mmol}, 1.0$ equiv), TIPS-EBX (1b) (112 mg, $0.260 \mathrm{mmol}, 1.30$ equiv), sat. $\mathrm{K}_{2} \mathrm{CO}_{3}$ solution $33 \% \mathrm{w} / \mathrm{w}$ (1.0 $\mathrm{mL}$ ) and Maruoka's catalyst 12 (4.49 mg, $6.00 \mu \mathrm{mol}, 0.0300$ equiv). The reaction was finished after $18 \mathrm{~h}$ at $10{ }^{\circ} \mathrm{C}$. The crude product was purified via silica gel flash chromatography (Hexane/EtOAc 20:1) to afford 3b' $(54.4 \mathrm{mg}, 0.132 \mathrm{mmol}, 66 \%)$ as yellow oil. For HPLC analysis the product 3b' was deprotected by the addition of TBAF $(145 \mu \mathrm{L}$, 1.10 equiv) in $0.5 \mathrm{~mL}$ of THF and stirring $1 \mathrm{~h}$ at RT. HPLC analysis with a Daicel Chiralpack IC column; $20{ }^{\circ} \mathrm{C}$; 0 to $32 \mathrm{~min}$; flow rate $1.0 \mathrm{~mL} / \mathrm{min}$; $\left[{ }^{i} \mathrm{PrOH} /\right.$ hexane, 0 to $5 \mathrm{~min} 5 / 95,5$ to 25 $\min 5 / 95$ to $30 / 70,25 \min$ to $27 \mathrm{~min} 30 / 70$ to $5 / 95,27 \min$ to $32 \mathrm{~min} 5 / 95]$; $\tau_{\text {minor }}=16.4 \mathrm{~min}$, $\tau_{\text {major }}=18.2 \mathrm{~min}$, gave $64 \%$ ee for $\mathbf{3 b}$.

$\mathrm{R}_{f} 0.71$ (PET/EtOAc 4:1, UV/Anisaldehyde). ${ }^{1} \mathrm{H}$ NMR $\left(400 \mathrm{MHz}, \mathrm{CDCl}_{3}\right) \delta 7.80$ (dd, $J=$ 7.7, $0.4 \mathrm{~Hz}, 1 \mathrm{H}$; ArH), $7.63(\mathrm{td}, J=7.6,1.2 \mathrm{~Hz}, 1 \mathrm{H} ; \operatorname{Ar} H), 7.47(\mathrm{~m}, 1 \mathrm{H} ; \mathrm{Ar} H), 7.40(\mathrm{~m}, 1 \mathrm{H}$; $\operatorname{ArH}), 3.89\left(\mathrm{~d}, J=17.0 \mathrm{~Hz}, 1 \mathrm{H} ; \mathrm{CH}_{2}\right), 3.44\left(\mathrm{~d}, J=17.0 \mathrm{~Hz}, 1 \mathrm{H} ; \mathrm{CH}_{2}\right), 1.45(\mathrm{~s}, 9 \mathrm{H}$; $\left.\mathrm{OC}\left(\mathrm{CH}_{3}\right)_{3}\right), 1.05(\mathrm{~m}, 18 \mathrm{H}$; TIPS $) .{ }^{13} \mathrm{C}$ NMR $\left(101 \mathrm{MHz}, \mathrm{CDCl}_{3}\right) \delta 196.3,167.0,152.2,135.5$, 133.6, 127.9, 126.3, 125.5, 103.7, 84.5, 83.1, 57.3, 41.1, 27.7, 18.6, 11.2. IR v 2943 (m), 2866 (m), 2723 (w), 2257 (w), $2171(\mathrm{w}), 1725$ (s), 1609 (m), 1592 (w), 1464 (m), 1394 (w), 1370 (m), $1285(\mathrm{~m}), 1254(\mathrm{~m}), 1212(\mathrm{w}), 1152(\mathrm{~s}), 1072(\mathrm{w}), 996(\mathrm{~m}), 907(\mathrm{~s}), 884(\mathrm{~m}), 844(\mathrm{~m})$, 812 (w), 742 (s), 679 (s), 652 (s). HRMS(ESI) calcd for $\mathrm{C}_{25} \mathrm{H}_{36} \mathrm{O}_{3} \mathrm{SiAg}^{+}\left(\mathrm{M}+\mathrm{Ag}^{106.9}\right)$ 519.1485, found 519.1494; calcd for $\mathrm{C}_{25} \mathrm{H}_{36} \mathrm{O}_{3} \mathrm{SiAg}^{+}\left(\mathrm{M}+\mathrm{Ag}^{108.9}\right)$ 521.1484, found 521.1501.

2-Phenylpropan-2-yl 2-ethynyl-1-oxo-2,3-dihydro-1H-indene-2-carboxylate (95)

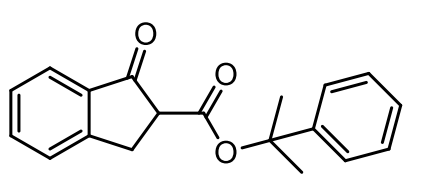

2c

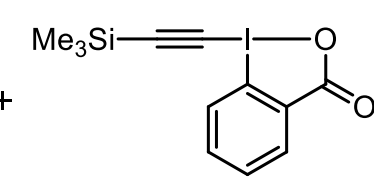

$1 \mathbf{a}$

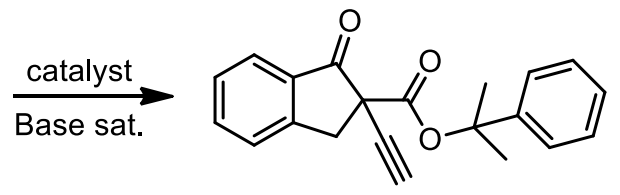

$3 c$

Following general procedure GP6, on a $0.08 \mathrm{mmol}$ scale using 2c $(23.7 \mathrm{mg}, 0.0800 \mathrm{mmol}$, 1.00 equiv) and TMS-EBX (1a) $(36.0 \mathrm{mg}, 0.105 \mathrm{mmol}, 1.30$ equiv). The reaction was finished after $3 \mathrm{~h}$ at $-78{ }^{\circ} \mathrm{C}$. Purification by flash chromatography (Hexane/EtOAc 20:1) afforded 3c (18.7 mg, $0.0587 \mathrm{mmol}, 73 \%)$ as yellow oil. 
Following general procedure GP8, on a $0.20 \mathrm{mmol}$ scale using $2 \mathbf{c}(58.8 \mathrm{mg}, 0.200 \mathrm{mmol}$, 1.00 equiv), TMS-EBX (1a) (89.5 mg, $0.260 \mathrm{mmol}, 1.30$ equiv), a sat. KF solution ( $33 \% \mathrm{w} / \mathrm{w}$, $1.0 \mathrm{~mL}$ ) and Maruoka's catalyst $12(4.49 \mathrm{mg}, 6.00 \mu \mathrm{mol}, 0.0300$ equiv), the reaction was finished after $18 \mathrm{~h}$ at RT. The crude product was purified via silica gel flash chromatography (Hexane/EtOAc 20:1) to afford 3c $(52.6 \mathrm{mg}, 0.165 \mathrm{mmol}, 83 \%)$ as a yellow oil. HPLC analysis with a Daicel Chiralpack IC column; $20{ }^{\circ} \mathrm{C}$; 0 to $32 \mathrm{~min}$; flow rate $1.0 \mathrm{~mL} / \mathrm{min}$; [ ${ }^{\mathrm{P} O \mathrm{OH}} / \mathrm{hexane}, 0$ to $5 \mathrm{~min} \mathrm{5/95,} 5$ to $25 \mathrm{~min} \mathrm{5/95}$ to 30/70, $25 \mathrm{~min}$ to $27 \mathrm{~min} \mathrm{30/70}$ to 5/95, 27 $\min$ to $32 \mathrm{~min} 5 / 95] ; \tau_{\text {minor }}=16.0 \mathrm{~min}, \tau_{\text {major }}=20.2 \mathrm{~min}$, gave $79 \%$ ee for $3 \mathbf{c}$. Optical rotation: $[\alpha]_{\mathrm{D}}^{25}=-4.3\left(\mathrm{c}=0.085, \mathrm{CH}_{2} \mathrm{Cl}_{2}\right)$.

$\mathrm{R}_{f} 0.4$ (PET/EtOAc 4:1, UV/Anisaldehyde). ${ }^{1} \mathrm{H}$ NMR (400 MHz, $\left.\mathrm{CDCl}_{3}\right) \delta 7.86(\mathrm{~m}, 1 \mathrm{H}$; $\operatorname{Ar} H), 7.68(\mathrm{td}, J=7.7,1.1 \mathrm{~Hz}, 1 \mathrm{H} ; \operatorname{Ar} H), 7.47(\mathrm{~m}, 2 \mathrm{H} ; \operatorname{Ar} H), 7.29(\mathrm{~m}, 5 \mathrm{H} ; \operatorname{Ar} H), 3.89(\mathrm{~d}, J$ $\left.=17.1 \mathrm{~Hz}, 1 \mathrm{H} ; \mathrm{CH}_{2}\right), 3.52\left(\mathrm{~d}, J=17.1 \mathrm{~Hz}, 1 \mathrm{H} ; \mathrm{CH}_{2}\right), 2.46(\mathrm{~s}, 1 \mathrm{H} ; \mathrm{C} \equiv \mathrm{CH}), 1.80(\mathrm{~d}, J=5.7$ $\mathrm{Hz}, 6 \mathrm{H}$; $\left.\left(\mathrm{CH}_{3}\right)_{2}\right) .{ }^{13} \mathrm{C} \mathrm{NMR}\left(101 \mathrm{MHz}, \mathrm{CDCl}_{3}\right) \delta 196.2,165.8,152.1,144.9,135.8,133.5$, $128.3,128.1,127.2,126.3,125.7,124.1,84.6,80.2,72.2,56.2,40.3,28.1$. IR $v 3440(\mathrm{w})$, $3289(\mathrm{w}), 3090(\mathrm{w}), 3067(\mathrm{w}), 2983(\mathrm{w}), 2930(\mathrm{w}), 2855(\mathrm{w}), 2252(\mathrm{w}), 2142(\mathrm{w}), 1724(\mathrm{~s})$, 1608 (m), 1498 (w), 1465 (m), 1432 (w), 1368 (w), 1254 (m), 1213 (m), 1134 (m), 1102 (m), 1078 (w), $1032(\mathrm{w}), 910(\mathrm{~s}), 839(\mathrm{~m}), 765(\mathrm{~m}), 733(\mathrm{~m}), 700(\mathrm{~m}), 650(\mathrm{~m}), 635$ (s). HRMS(ESI) calcd for $\mathrm{C}_{21} \mathrm{H}_{18} \mathrm{O}_{3} \mathrm{Ag}^{+}\left(\mathrm{M}+\mathrm{Ag}^{106.9}\right.$ ) 425.0307, found 425.0316; calcd for $\mathrm{C}_{21} \mathrm{H}_{18} \mathrm{O}_{3} \mathrm{Ag}^{+}\left(\mathrm{M}+\mathrm{Ag}^{108.9}\right)$ 427.0306, found 427.0327.

\section{1-Adamantan-1-yl 2-ethynyl-1-oxo-2,3-dihydro-1H-indene-2-carboxylate (3d)}

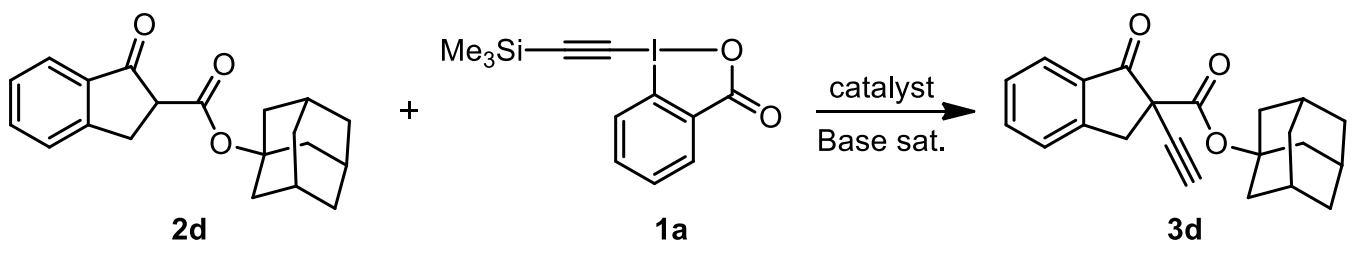

Following general procedure GP6, on a $0.08 \mathrm{mmol}$ scale using $2 \mathbf{d}(25.0 \mathrm{mg}, 0.0800 \mathrm{mmol}$, 1.00 equiv) and TMS-EBX (1a) $(36.0 \mathrm{mg}, 0.105 \mathrm{mmol}, 1.30$ equiv). The reaction was finished after $3.5 \mathrm{~h}$ at $-78{ }^{\circ} \mathrm{C}$. Purification by flash chromatography (Hexane/EtOAc 20:1) afforded 3d (20.0 mg, $0.0598 \mathrm{mmol}, 74 \%)$ as yellow oil.

Following general procedure GP8, on a $0.20 \mathrm{mmol}$ scale using $2 \mathbf{d}(62.0 \mathrm{mg}, 0.200 \mathrm{mmol}$, 1.00 equiv), TMS-EBX (1a) $(89.5 \mathrm{mg}, 0.260 \mathrm{mmol}, 1.30$ equiv), a sat. KF solution (33\% w/w, $1.0 \mathrm{~mL}$ ) and Maruoka's catalyst $12(4.49 \mathrm{mg}, 6.00 \mu \mathrm{mol}, 0.0300$ equiv), the reaction was finished after $18 \mathrm{~h}$ at RT. The crude product was purified via silica gel flash chromatography (Hexane/EtOAc 10:1) to afford 3d $(66.2 \mathrm{mg}, 0.198 \mathrm{mmol}, 99 \%)$ as yellow oil. HPLC analysis with a Daicel Chiralpack IC column; $20{ }^{\circ} \mathrm{C}$; 0 to $32 \mathrm{~min}$; flow rate $1.0 \mathrm{~mL} / \mathrm{min}$; [ ${ }^{\mathrm{PrOH}} / \mathrm{hexane}, 0$ to $5 \mathrm{~min} \mathrm{5/95,} 5$ to $25 \mathrm{~min} \mathrm{5/95}$ to 30/70, $25 \mathrm{~min}$ to $27 \mathrm{~min} \mathrm{30/70}$ to 5/95, 27 min to $32 \mathrm{~min} 5 / 95]$; $\tau_{\text {minor }}=19.3 \mathrm{~min}, \tau_{\text {major }}=21.5 \mathrm{~min}$, gave $55 \%$ ee for $3 \mathbf{d}$. Optical rotation: $[\alpha]_{\mathrm{D}}^{25}=+3.7\left(\mathrm{c}=0.083, \mathrm{CH}_{2} \mathrm{Cl}_{2}\right)$.

$\mathrm{R}_{f} 0.4$ (PET/EtOAc 4:1, UV/Anisaldehyde). ${ }^{1} \mathrm{H}$ NMR (400 MHz, $\left.\mathrm{CDCl}_{3}\right) \delta 7.82(\mathrm{~d}, J=7.6$ $\mathrm{Hz} ; 1 \mathrm{H}$; ArH), 7.65 (m, $1 \mathrm{H}$; ArH), $7.48(\mathrm{~d}, J=7.7 \mathrm{~Hz}, 1 \mathrm{H}$; $\operatorname{Ar} H), 7.43$ (m, $1 \mathrm{H} ; \operatorname{Ar} H), 3.84$ $\left(\mathrm{d}, J=17.0 \mathrm{~Hz}, 1 \mathrm{H} ; \mathrm{CH}_{2}\right), 3.49\left(\mathrm{~d}, J=17.0 \mathrm{~Hz}, 1 \mathrm{H} ; \mathrm{CH}_{2}\right), 2.39$ (s, $\left.1 \mathrm{H} ; \mathrm{C} \equiv \mathrm{CH}\right), 2.16(\mathrm{~s}, 2 \mathrm{H}$; $\left.(\mathrm{CH})_{2}\right), 2.08\left(\mathrm{~d}, J=3.0 \mathrm{~Hz}, 6 \mathrm{H} ;\left(\mathrm{CH}_{2}\right)_{3}\right), 1.64\left(\mathrm{t}, J=2.9 \mathrm{~Hz}, 7 \mathrm{H} ;\left(\mathrm{CH}_{2} \mathrm{CH}\right)_{3}\right) .{ }^{13} \mathrm{C} \mathrm{NMR}(101$ $\left.\mathrm{MHz}, \mathrm{CDCl}_{3}\right) \delta 196.6,166.2,152.2,135.7,133.5,128.0,126.2,125.6,83.5,80.3,72.1,56.3$, 40.8, 40.7, 35.9, 30.8. IR v 3676 (w), 3307 (w), 3281 (w), 2912 (s), 2853 (m), 2256 (w), 2147 
(w), $1723(\mathrm{~s}), 1607(\mathrm{~m}), 1591(\mathrm{w}), 1458(\mathrm{~m}), 1432(\mathrm{w}), 1355(\mathrm{w}), 1250(\mathrm{~s}), 1213(\mathrm{~m}), 1188$ (m), 1155 (w), 1104 (w), 1049 (s), 965 (w), 909 (s), 865 (w), 800 (m), 732 (s), 694 (w), 633 (m). HRMS (ESI) calcd for $\mathrm{C}_{22} \mathrm{H}_{23} \mathrm{O}_{3}{ }^{+}(\mathrm{M}+\mathrm{H})$ 335.1647, found 335.1651.

\section{Anthracen-9-ylmethyl 2-ethynyl-1-oxo-2,3-dihydro-1H-indene-2-carboxylate (3e)}
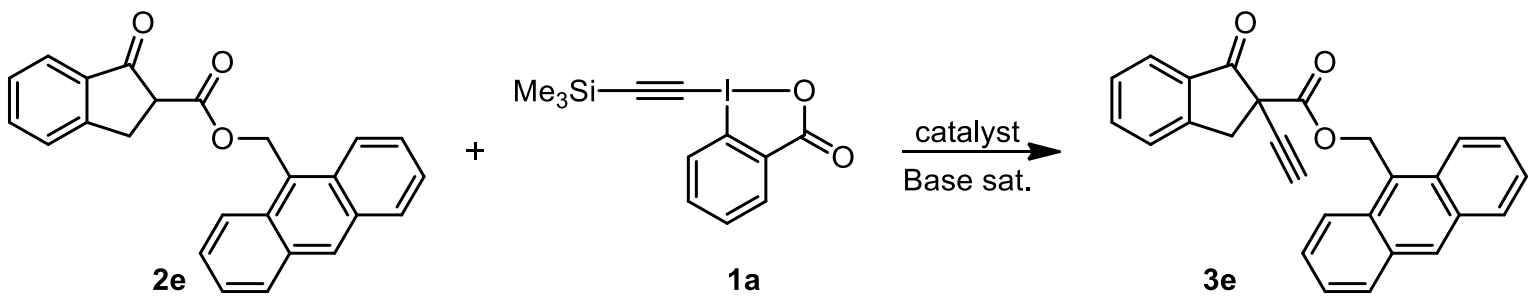

Following general procedure GP6, on a $0.08 \mathrm{mmol}$ scale using $\mathbf{2 e}(29.6 \mathrm{mg}, 0.0800 \mathrm{mmol}$, 1.00 equiv) and TMS-EBX (1a) $(36.0 \mathrm{mg}, 0.105 \mathrm{mmol}, 1.30$ equiv). The reaction was finished after $5 \mathrm{~h}$ at $-78{ }^{\circ} \mathrm{C}$. Purification by flash chromatography (Hexane/EtOAc 10:1) afforded 3e (32.2 mg, $0.0825 \mathrm{mmol}, 98 \%)$ as yellow solid.

Following general procedure GP8, on a $0.20 \mathrm{mmol}$ scale using $2 \mathbf{e}(73.3 \mathrm{mg}, 0.200 \mathrm{mmol}$, 1.00 equiv), TMS-EBX (1a) $(89.5 \mathrm{mg}, 0.260 \mathrm{mmol}, 1.30$ equiv), a sat. KF solution ( $33 \% \mathrm{w} / \mathrm{w}$, $1.0 \mathrm{~mL}$ ) and Maruoka's catalyst 12 (4.49 mg, $6.00 \mu \mathrm{mol}, 0.0300$ equiv), the reaction was finished after $18 \mathrm{~h}$ at RT. The crude product was purified via silica gel flash chromatography (Hexane/EtOAc 10:1) to afford 3e $(65.7 \mathrm{mg}, 0.168 \mathrm{mmol}, 84 \%)$ as a yellow solid. HPLC analysis with a Daicel Chiralpack IC column; $20{ }^{\circ} \mathrm{C}$; 0 to $44 \mathrm{~min}$; flow rate $1.0 \mathrm{~mL} / \mathrm{min}$; [ ${ }^{i} \mathrm{PrOH} /$ hexane, 0 to $5 \mathrm{~min} 5 / 95,5$ to $10 \mathrm{~min} \mathrm{5/95}$ to 7/93, $10 \mathrm{~min}$ to $40 \mathrm{~min} 7 / 93$ to $7 / 93,40$ min to $42 \mathrm{~min} 5 / 95] ; \tau_{\text {minor }}=28.8 \mathrm{~min}, \tau_{\text {major }}=30.6 \mathrm{~min}$, gave $27 \%$ ee for 3e. Optical rotation: $[\alpha]_{\mathrm{D}}^{25}=+14.7\left(\mathrm{c}=0.083, \mathrm{CH}_{2} \mathrm{Cl}_{2}\right)$.

$\mathrm{R}_{f} 0.61$ (PET/EtOAc 4:1, UV/Anisaldehyde). ${ }^{1} \mathrm{H}$ NMR $\left(400 \mathrm{MHz}, \mathrm{CDCl}_{3}\right) \delta 8.49(\mathrm{~s}, 1 \mathrm{H}$; $\operatorname{ArH}), 8.27(\mathrm{~d}, J=8.7 \mathrm{~Hz}, 2 \mathrm{H} ; \operatorname{Ar} H), 8.00(\mathrm{~d}, J=8.1 \mathrm{~Hz}, 2 \mathrm{H} ; \operatorname{ArH}), 7.79(\mathrm{~d}, J=7.7 \mathrm{~Hz}, 1 \mathrm{H}$; $\operatorname{Ar} H), 7.58(\mathrm{t}, J=7.6 \mathrm{~Hz}, 1 \mathrm{H}$; $\operatorname{Ar} H), 7.49(\mathrm{~m}, 4 \mathrm{H}$; ArH), $7.36(\mathrm{~m}, 2 \mathrm{H}$; ArH), 6.28 (d, $J=$ $\left.12.6 \mathrm{~Hz}, 1 \mathrm{H} ; \mathrm{OCH}_{2}\right), 6.17\left(\mathrm{~d}, J=12.6 \mathrm{~Hz}, 1 \mathrm{H} ; \mathrm{OCH}_{2}\right), 3.70\left(\mathrm{~d}, J=17.1 \mathrm{~Hz}, 1 \mathrm{H} ; \mathrm{CH}_{2}\right), 3.41$ $\left(\mathrm{d}, J=17.1 \mathrm{~Hz}, 1 \mathrm{H} ; \mathrm{CH}_{2}\right), 2.32$ (s, $\left.1 \mathrm{H} ; \mathrm{C} \equiv \mathrm{CH}\right) .{ }^{13} \mathrm{C} \mathrm{NMR}\left(101 \mathrm{MHz}, \mathrm{CDCl}_{3}\right) \delta 195.8,168.0$, $152.0,135.8,133.2,131.2,131.0,129.5,128.9,128.1,126.6,126.3,125.7,125.2,125.0$, 123.9, 79.6, 72.6, 61.4, 55.5, 40.5. IR v $3292(\mathrm{w}), 3056(\mathrm{w}), 2987(\mathrm{w}), 2881(\mathrm{w}), 2843(\mathrm{w})$, $2181(\mathrm{w}), 2156(\mathrm{w}), 1746$ (s), 1722 (s), 1607 (w), $1527(\mathrm{w}), 1477$ (w), $1432(\mathrm{w}), 1377$ (w), $1259(\mathrm{~m}), 1211(\mathrm{~m}), 1186(\mathrm{~s}), 1066(\mathrm{~m}), 908$ (s), $866(\mathrm{w}), 800(\mathrm{w}), 734(\mathrm{~s}), 689(\mathrm{~m}), 649(\mathrm{~m})$, 638 (m). HRMS(ESI) calcd for $\mathrm{C}_{27} \mathrm{H}_{18} \mathrm{O}_{3} \mathrm{Ag}^{+}\left(\mathrm{M}+\mathrm{Ag}^{106.9}\right)$ 497.0307, found 497.0329; calcd for $\mathrm{C}_{27} \mathrm{H}_{18} \mathrm{O}_{3} \mathrm{Ag}^{+}\left(\mathrm{M}+\mathrm{Ag}^{108.9}\right)$ 499.0307, found 499.0328.

\section{2-Ethynyl-1-oxo-N-phenyl-2,3-dihydro-1H-indene-2-carboxamide (3f)}

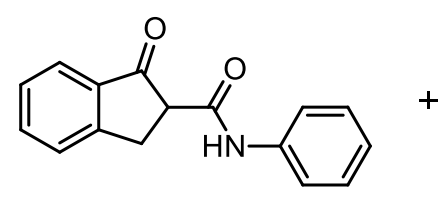

$2 f$

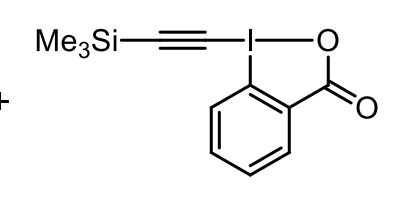

$1 \mathrm{a}$
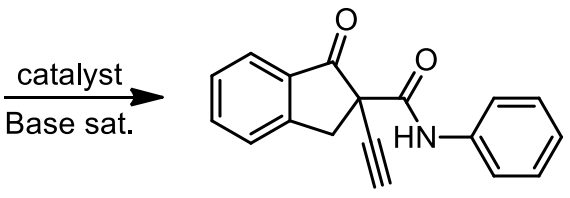

$3 f$

Following general procedure GP6, on a $0.08 \mathrm{mmol}$ scale using $2 \mathbf{f}(20.0 \mathrm{mg}, 0.0800 \mathrm{mmol}$, 1.00 equiv) and TMS-EBX (1a) $(36.0 \mathrm{mg}, 0.105 \mathrm{mmol}, 1.30$ equiv). The reaction was 
finished after $2.5 \mathrm{~h}$ at $-78{ }^{\circ} \mathrm{C}$. Purification by flash chromatography (Hexane/EtOAc 10:1) afforded $3 f(14.5 \mathrm{mg}, 0.0527 \mathrm{mmol}, 65 \%)$ as yellow oil.

Following general procedure GP8, on a $0.20 \mathrm{mmol}$ scale using $\mathbf{2 f}$ (50.0 $\mathrm{mg}, 0.200 \mathrm{mmol}$, 1.00 equiv), TMS-EBX (1a) $(89.5 \mathrm{mg}, 0.260 \mathrm{mmol}, 1.30$ equiv), a sat. KF solution $(33 \% \mathrm{w} / \mathrm{w}$, $1.0 \mathrm{~mL}$ ) and Maruoka's catalyst $12(4.49 \mathrm{mg}, 6.00 \mu \mathrm{mol}, 0.0300$ equiv), the reaction was finished after $18 \mathrm{~h}$ at RT. The crude product was purified via silica gel flash chromatography (Hexane/EtOAc 10:1) to afford $\mathbf{3 f}(31.5 \mathrm{mg}, 0.114 \mathrm{mmol}, 57 \%)$ as yellow oil. HPLC analysis with a Daicel Chiralpack IC column; $20{ }^{\circ} \mathrm{C}$; 0 to $32 \mathrm{~min}$; flow rate $1.0 \mathrm{~mL} / \mathrm{min}$; [ ${ }^{\mathrm{PrOH}} / \mathrm{h}$ exane, 0 to $5 \mathrm{~min} 5 / 95,5$ to $25 \mathrm{~min} 5 / 95$ to 30/70, $25 \mathrm{~min}$ to $27 \mathrm{~min}$ 30/70 to 5/95, 27 $\min$ to $32 \mathrm{~min} 5 / 95] ; \tau_{\text {minor }}=16.6 \mathrm{~min}, \tau_{\mathrm{major}}=22.7 \mathrm{~min}$, gave $16 \%$ ee for $\mathbf{3 f}$.

$\mathrm{R}_{f} 0.27$ (PET/EtOAc 4:1, UV/Anisaldehyde). ${ }^{1} \mathrm{H}$ NMR (400 MHz, $\left.\mathrm{CDCl}_{3}\right) \delta 8.67$ (br s, 1 $\mathrm{H} ; \mathrm{NH}), 7.84(\mathrm{~d}, J=7.6 \mathrm{~Hz}, 1 \mathrm{H} ; \operatorname{Ar} H), 7.71(\mathrm{t}, J=7.5 \mathrm{~Hz}, 1 \mathrm{H} ; \operatorname{Ar} H), 7.58(\mathrm{~m}, 2 \mathrm{H} ; \operatorname{Ar} H)$, $7.46(\mathrm{t}, J=7.6 \mathrm{~Hz}, 1 \mathrm{H} ; \mathrm{ArH}), 7.36(\mathrm{t}, J=7.6 \mathrm{~Hz}, 2 \mathrm{H} ; \operatorname{Ar} H), 7.28(\mathrm{~d}, J=0.9 \mathrm{~Hz}, 1 \mathrm{H} ; \operatorname{Ar} H)$, $7.16(\mathrm{~m}, 1 \mathrm{H} ; \mathrm{ArH}), 4.28$ (d, J=17.2 Hz, $\left.1 \mathrm{H} ; \mathrm{CH}_{2}\right), 3.50$ (d, J=17.2 Hz, $\left.1 \mathrm{H} ; \mathrm{CH}_{2}\right), 2.60$ (s, $1 \mathrm{H} ; \mathrm{C} \equiv \mathrm{CH}) .{ }^{13} \mathrm{C}$ NMR $\left(101 \mathrm{MHz}, \mathrm{CDCl}_{3}\right) \delta 198.3,163.3,153.4,137.3,136.4,132.9,129.0$, 128.1, 126.5, 125.7, 124.8, 119.9, 81.3, 73.5, 56.2, 38.5. IR $v 3042(\mathrm{w}), 3022(\mathrm{w}), 2986(\mathrm{w})$, $2946(\mathrm{w}), 2923(\mathrm{w}), 2866(\mathrm{w}), 2358(\mathrm{w}), 2342(\mathrm{w}), 1784(\mathrm{w}), 1730(\mathrm{~m}), 1685(\mathrm{w}), 1601(\mathrm{w})$, 1530 (s), 1489 (m), 1445 (w), 1380 (w), 1315 (w), 1272 (m), 1203 (w), 1156 (w), 1061 (w), $993(\mathrm{w}), 927(\mathrm{~m}), 904(\mathrm{~m}), 859(\mathrm{w}), 765(\mathrm{~s}), 727(\mathrm{~m}), 647(\mathrm{~s})$. HRMS (ESI) calcd for $\mathrm{C}_{18} \mathrm{H}_{14} \mathrm{NO}_{2}^{+}(\mathrm{M}+\mathrm{H}) 276.1024$, found 276.1028 .

\section{2-Ethynyl- $N$-(4-fluorophenyl)-1-oxo-2,3-dihydro- $1 H$-indene-2-carboxamide (3g)}

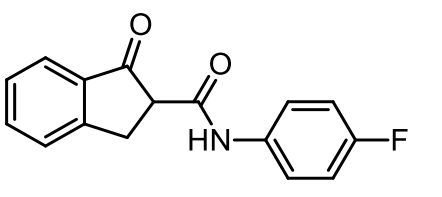

$2 \mathrm{~g}$

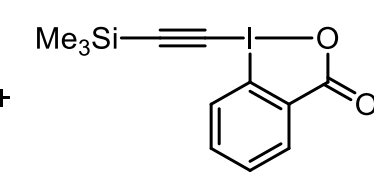

1a

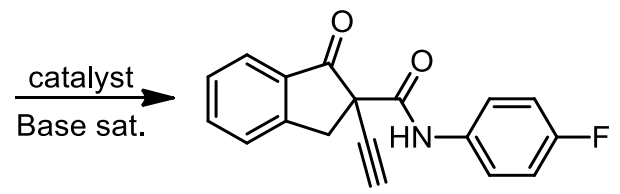

$3 \mathbf{g}$

Following general procedure GP6, on a $0.08 \mathrm{mmol}$ scale using $\mathbf{2 g}$ ( $21.7 \mathrm{mg}, 0.0800 \mathrm{mmol}$, 1.00 equiv) and TMS-EBX (1a) $(36.0 \mathrm{mg}, 0.105 \mathrm{mmol}, 1.30$ equiv). The reaction was finished after $1 \mathrm{~h}$ at $-78{ }^{\circ} \mathrm{C}$. Purification by flash chromatography (Hexane/EtOAc 5:1) afforded $\mathbf{3 g}$ (19.6 mg, $0.0668 \mathrm{mmol}, 83 \%)$ as yellow oil.

Following general procedure GP8, on a $0.20 \mathrm{mmol}$ scale using $2 \mathrm{~g}$ (53.8 $\mathrm{mg}, 0.200 \mathrm{mmol}$, 1.00 equiv), TMS-EBX (1a) $(89.5 \mathrm{mg}, 0.260 \mathrm{mmol}, 1.30$ equiv), a sat. KF solution (33\% w/w, $1.0 \mathrm{~mL}$ ) and Maruoka's catalyst 12 (4.49 mg, $6.00 \mu \mathrm{mol}, 0.0300$ equiv), the reaction was finished after $18 \mathrm{~h}$ at RT. The crude product was purified via silica gel flash chromatography (Hexane/EtOAc 5:1) to afford $\mathbf{3 g}(54.5 \mathrm{mg}, 0.186 \mathrm{mmol}, 93 \%)$ as yellow oil. HPLC analysis with a Daicel Chiralpack IC column; $20{ }^{\circ} \mathrm{C}$; 0 to $32 \mathrm{~min}$; flow rate $1.0 \mathrm{~mL} / \mathrm{min}$; [ ${ }^{i} \mathrm{PrOH} / \mathrm{h}$ exane, 0 to $5 \mathrm{~min} 5 / 95,5$ to $25 \mathrm{~min} 5 / 95$ to 30/70, $25 \mathrm{~min}$ to $27 \mathrm{~min} 30 / 70$ to 5/95, 27 min to $32 \mathrm{~min} 5 / 95]$; $\tau_{\text {minor }}=14.0 \mathrm{~min}, \tau_{\text {major }}=17.6 \mathrm{~min}$, gave $30 \%$ ee for $3 \mathrm{~g}$. Optical rotation: $[\alpha]_{\mathrm{D}}^{25}=+27.8\left(\mathrm{c}=0.083, \mathrm{CH}_{2} \mathrm{Cl}_{2}\right)$.

$\mathrm{R}_{f} 0.72$ (PET/EtOAc 1:1, UV/Anisaldehyde). ${ }^{1} \mathrm{H}$ NMR $\left(400 \mathrm{MHz}, \mathrm{CDCl}_{3}\right) \delta 8.63$ (br s, 1 $\mathrm{H} ; \mathrm{NH}), 7.81(\mathrm{~d}, J=7.7 \mathrm{~Hz}, 1 \mathrm{H} ; \operatorname{Ar} H), 7.69(\mathrm{t}, J=7.6 \mathrm{~Hz}, 1 \mathrm{H} ; \operatorname{Ar} H), 7.52(\mathrm{~m}, 3 \mathrm{H} ; \operatorname{Ar} H)$, $7.43(\mathrm{t}, J=7.6 \mathrm{~Hz}, 1 \mathrm{H} ; \mathrm{ArH}), 7.02(\mathrm{t}, J=8.5 \mathrm{~Hz}, 2 \mathrm{H} ; \mathrm{ArH}), 4.23\left(\mathrm{~d}, J=17.2 \mathrm{~Hz}, 1 \mathrm{H} ; \mathrm{CH}_{2}\right)$, $3.47\left(\mathrm{~d}, J=17.2 \mathrm{~Hz}, 1 \mathrm{H} ; \mathrm{CH}_{2}\right), 2.57(\mathrm{~s}, 1 \mathrm{H} ; \mathrm{C} \equiv \mathrm{CH}) .{ }^{13} \mathrm{C}$ NMR $\left(101 \mathrm{MHz}, \mathrm{CDCl}_{3}\right) \delta 198.2$, 163.4, 160.9, 158.4, 153.3, 136.4, $133.4(\mathrm{~d}, J(\mathrm{C}, \mathrm{F})=3 \mathrm{~Hz}), 132.8,131.8,128.2,126.5,125.7$, 
$121.8(\mathrm{~d}, J(\mathrm{C}, \mathrm{F})=8 \mathrm{~Hz}), 115.7(\mathrm{~d}, J(\mathrm{C}, \mathrm{F})=23 \mathrm{~Hz}), 81.3,73.6,56.0,38.4$. IR $v 3394(\mathrm{w})$, $3301(\mathrm{w}), 3076(\mathrm{w}), 3048$ (w), 2937 (w), 2253 (w), 1726 (m), 1685 (m), 1609 (w), $1591(\mathrm{w})$, 1533 (m), 1510 (s), 1465 (w), 1409 (m), 1303 (w), 1273 (w), $1214(\mathrm{~m}), 1158(\mathrm{w}), 1101(\mathrm{w})$, $1015(\mathrm{w}), 993$ (w), 909 (s), $875(\mathrm{w}), 833(\mathrm{~m}), 808(\mathrm{~m}), 788(\mathrm{w}), 730(\mathrm{~s}), 690(\mathrm{~m}), 667(\mathrm{~m})$, $651(\mathrm{~m}), 639(\mathrm{~m})$. HRMS (ESI) calcd for $\mathrm{C}_{18} \mathrm{H}_{13} \mathrm{NO}_{2} \mathrm{~F}^{+}(\mathrm{M}+\mathrm{H}) 294.0930$, found 294.0930.

$N$-(3,5-bis(trifluoromethyl)phenyl)-2-ethynyl-1-oxo-2,3-dihydro-1 $H$-indene-2carboxamide (3h)<smiles>O=C(Nc1cc(C(F)(F)F)cc(C(F)(F)F)c1)C1Cc2ccccc2C1=O</smiles>

$2 \mathrm{~h}$<smiles>C[SiH2]C#CI1C(=O)OC1=C1C=CC=CC1</smiles>

$1 \mathrm{a}$<smiles>C#CC1(C(=O)Nc2cc(C(F)(F)F)cc(C(F)(F)F)c2)Cc2ccccc2C1=O</smiles>

$3 \mathrm{~h}$

Following general procedure GP6, on a $0.08 \mathrm{mmol}$ scale using $\mathbf{2 h}(31.2 \mathrm{mg}, 0.0800 \mathrm{mmol}$, 1.00 equiv) and TMS-EBX (1a) $(36.0 \mathrm{mg}, 0.105 \mathrm{mmol}, 1.30$ equiv). The reaction was finished after $1 \mathrm{~h}$ at $-78{ }^{\circ} \mathrm{C}$. Purification by flash chromatography (Hexane/EtOAc 10:1) afforded $\mathbf{3 h}$ (15.9 mg, $0.0387 \mathrm{mmol}, 50 \%)$ as yellow solid.

Following general procedure GP8, on a $0.20 \mathrm{mmol}$ scale using $\mathbf{2 h}(77.4 \mathrm{mg}, 0.200 \mathrm{mmol}$, 1.00 equiv), TMS-EBX (1a) ( $89.5 \mathrm{mg}, 0.260 \mathrm{mmol}, 1.30$ equiv), a sat. KF solution ( $33 \% \mathrm{w} / \mathrm{w}$, $1.0 \mathrm{~mL}$ ) and Maruoka's catalyst $12(4.49 \mathrm{mg}, 6.00 \mu \mathrm{mol}, 0.0300$ equiv), the reaction was finished after $18 \mathrm{~h}$ at RT. The crude product was purified via silica gel flash chromatography (Hexane/EtOAc 10:1) to afford $\mathbf{3 h}(42.0 \mathrm{mg}, 0.102 \mathrm{mmol}, 51 \%)$ as yellow solid. HPLC analysis in Daicel Chiralpack IC column; $20{ }^{\circ} \mathrm{C}$; 0 to $32 \mathrm{~min}$; flow rate $1.0 \mathrm{~mL} / \mathrm{min}$; [ ${ }^{\mathrm{PrOH}} / \mathrm{hexane}, 0$ to $5 \mathrm{~min} \mathrm{5/95,} 5$ to $25 \mathrm{~min} \mathrm{5/95}$ to 30/70, $25 \mathrm{~min}$ to $27 \mathrm{~min} \mathrm{30/70}$ to 5/95, 27 min to $32 \mathrm{~min} 5 / 95] ; \tau_{\text {minor }}=17.6 \mathrm{~min}, \tau_{\text {major }}=23.8 \mathrm{~min}$, gave $55 \%$ ee for $3 \mathbf{h}$. Optical rotation: $[\alpha]_{\mathrm{D}}^{25}=+22.0\left(\mathrm{c}=0.083, \mathrm{CH}_{2} \mathrm{Cl}_{2}\right)$.

$\mathrm{R}_{f} 0.6$ (PET/EtOAc 4:1, UV/Anisaldehyde). ${ }^{1} \mathrm{H}$ NMR (400 MHz, $\left.\mathrm{CDCl}_{3}\right) \delta 8.96(\mathrm{br} \mathrm{s}, 1 \mathrm{H}$; $\mathrm{NH}), 8.10(\mathrm{~s}, 2 \mathrm{H} ; \operatorname{Ar} H), 7.85(\mathrm{~d}, J=7.5 \mathrm{~Hz}, 1 \mathrm{H} ; \operatorname{Ar} H), 7.74(\mathrm{td}, J=7.7,1.2 \mathrm{~Hz}, 1 \mathrm{H} ; \operatorname{Ar} H)$, 7.66 (s, $1 \mathrm{H} ; \operatorname{ArH}), 7.59$ (dt, $J=7.7,0.7 \mathrm{~Hz}, 1 \mathrm{H}$; ArH), $7.48(\mathrm{~m}, 1 \mathrm{H} ; \mathrm{ArH}), 4.27$ (d, J= 17.3 $\left.\mathrm{Hz}, 1 \mathrm{H} ; \mathrm{CH}_{2}\right), 3.53\left(\mathrm{~d}, J=17.3 \mathrm{~Hz}, 1 \mathrm{H} ; \mathrm{CH}_{2}\right), 2.63(\mathrm{~s}, 1 \mathrm{H} ; \mathrm{C} \equiv \mathrm{CH}) .{ }^{13} \mathrm{C}$ NMR $(101 \mathrm{MHz}$, $\left.\mathrm{CDCl}_{3}\right) \delta 197.5,166.6,153.5,138.8,136.8,132.5(\mathrm{q}, J(\mathrm{C}, \mathrm{F})=34 \mathrm{~Hz}), 132.5,128.4,126.6$, $125.9,123.0(\mathrm{q}, J(\mathrm{C}, \mathrm{F})=273 \mathrm{~Hz}), 119.7(\mathrm{~m}), 118.2(\mathrm{~m}), 84.0,74.0,56.2,38.1 . \mathrm{IR} \vee 3392(\mathrm{w})$, $3356(\mathrm{w}), 3307(\mathrm{w}), 3111(\mathrm{w}), 3088(\mathrm{w}), 3076(\mathrm{w}), 2937(\mathrm{w}), 1726(\mathrm{~m}), 1699(\mathrm{~m}), 1607(\mathrm{w})$, 1547 (m), 1473 (m), 1470 (m), 1382 (s), 1278 (s), 1179 (s), 1134 (s), 1001 (w), 939 (w), 911 (m), $888(\mathrm{~m}), 806(\mathrm{w}), 783(\mathrm{w}), 736(\mathrm{~m}), 702(\mathrm{~m}), 684(\mathrm{~m}), 648(\mathrm{~m})$. HRMS (ESI) calcd for $\mathrm{C}_{20} \mathrm{H}_{12} \mathrm{NO}_{2} \mathrm{~F}_{6}{ }^{+}(\mathrm{M}+\mathrm{H})$ 412.0772, found 412.0777.

Tert-Butyl 1-oxo-2-(phenylethynyl)-2,3-dihydro-1H-indene-2-carboxylate (7a)

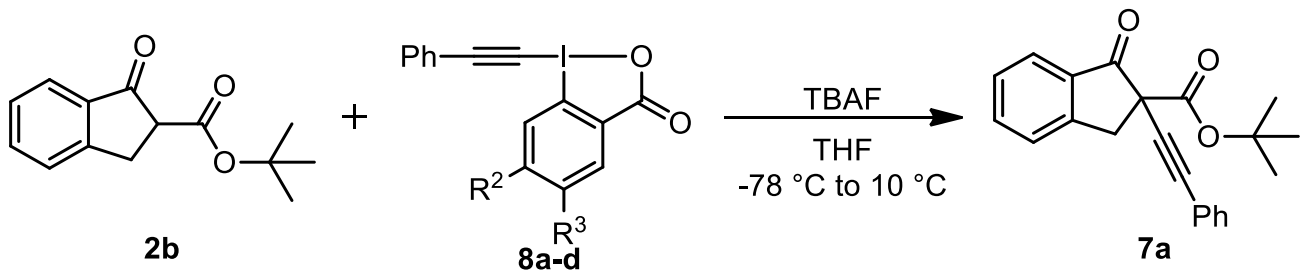


Following general procedure GP6, on a $0.08 \mathrm{mmol}$ scale using $\mathbf{2 b}(18.8 \mathrm{mg}, 0.0807 \mathrm{mmol}$, 1.0 equiv) and Ph-EBX (8a) (36.5 $\mathrm{mg}, 0.105 \mathrm{mmol}, 1.3$ equiv). The reaction was finished after $18 \mathrm{~h}$ at $10{ }^{\circ} \mathrm{C}$. Purification by flash chromatography (Hexane/EtOAc 10:1) afforded 7a (13.4 $\mathrm{mg}, 0.0403 \mathrm{mmol}, 48 \%$ ) as yellow solid.

Following general procedure GP6, on a $0.08 \mathrm{mmol}$ scale using $\mathbf{2 b}(18.8 \mathrm{mg}, 0.0807 \mathrm{mmol}$, 1.0 equiv) and 5-nitro-1-[phenylethynyl]-1,2-benziodoxol-3(1H)-one $(\mathbf{8 b})$ (41.2 mg, 0.105 mmol, 1.3 equiv). The reaction was finished after $18 \mathrm{~h}$ at $10{ }^{\circ} \mathrm{C}$. Purification by flash chromatography (Hexane/EtOAc 10:1) afforded 7a (6.80 mg, $0.0204 \mathrm{mmol}, 25 \%)$ as yellow solid.

Following general procedure GP6, on a $0.08 \mathrm{mmol}$ scale using $\mathbf{2 b}(18.8 \mathrm{mg}, 0.0807 \mathrm{mmol}$, 1.0 equiv) and 5-fluoro-1-[phenylethynyl]-1,2-benziodoxol-3(1H)-one (8c) $(38.4 \mathrm{mg}, 0.105$ mmol, 1.3 equiv). The reaction was finished after $18 \mathrm{~h}$ at $10{ }^{\circ} \mathrm{C}$. Purification by flash chromatography (Hexane/EtOAc 10:1) afforded 7a $(6.41 \mathrm{mg}, 0.0193 \mathrm{mmol}, 24 \%)$ as yellow solid.

Following general procedure GP6, on a $0.08 \mathrm{mmol}$ scale using $\mathbf{2 b}(18.8 \mathrm{mg}, 0.0807 \mathrm{mmol}$, 1.0 equiv) and 4,5-dimethoxy-1-[phenylethynyl]-1,2-benziodoxol-3(1H)-one $(\mathbf{8 d})(42.9 \mathrm{mg}$, $0.105 \mathrm{mmol}, 1.3$ equiv). The reaction was finished after $18 \mathrm{~h}$ at $10{ }^{\circ} \mathrm{C}$. Purification by flash chromatography (Hexane/EtOAc 10:1) afforded 7a (15.2 $\mathrm{mg}, 0.0457 \mathrm{mmol}, 57 \%)$ as yellow solid.

$\mathrm{R}_{f} 0.46$ (PET/EtOAc 4:1, UV/Anisaldehyde). ${ }^{1} \mathrm{H}$ NMR $\left(400 \mathrm{MHz}, \mathrm{CDCl}_{3}\right) \delta 7.85$ (d, $J=$ $7.7 \mathrm{~Hz}, 1 \mathrm{H} ; \operatorname{Ar} H), 7.66(\mathrm{td}, J=7.7,1.0 \mathrm{~Hz}, 1 \mathrm{H} ; \operatorname{Ar} H), 7.51(\mathrm{~d}, J=7.7 \mathrm{~Hz}, 1 \mathrm{H} ; \operatorname{Ar} H), 7.43$ $(\mathrm{m}, 3 \mathrm{H} ; \operatorname{ArH}), 7.27(\mathrm{~m}, 3 \mathrm{H} ; \operatorname{Ar} H), 3.91\left(\mathrm{~d}, J=17.1 \mathrm{~Hz}, 1 \mathrm{H} ; \mathrm{CH}_{2}\right), 3.58(\mathrm{~d}, J=17.1 \mathrm{~Hz}, 1 \mathrm{H}$; $\left.\mathrm{CH}_{2}\right), 1.46\left(\mathrm{~s}, 9 \mathrm{H}\right.$; $\left.\mathrm{OC}\left(\mathrm{CH}_{3}\right)_{3}\right) .{ }^{13} \mathrm{C} \mathrm{NMR}\left(101 \mathrm{MHz}, \mathrm{CDCl}_{3}\right) \delta 197.0,167.2,152.3,135.6$, $133.7,131.9,128.3,128.1,128.0,126.3,125.6,122.7,85.8,83.3,56.9,41.1,29.7,27.7$. IR v $3745(w), 3706(w), 3680(w), 3648(w), 3623(w), 3596(w), 3041(w), 2995(w), 2982(w)$, 2949 (w), 2918 (w), 2896 (w), 2878 (w), 2858 (w), 2364 (w), 2349 (w), 2334 (w), 1730 (m), 1607 (w), 1490 (w), 1462 (w), $1427(w), 1399(w), 1371(w), 1269(w), 1238(w), 1213(w)$, 1155 (m), 1095 (m), 1071 (w), 1061 (m), 1033 (w), 1020 (w), $921(\mathrm{w}), 848(\mathrm{w}), 758(\mathrm{w}), 692$ (w), 666 (w), 652 (s), 641 (s), 640 (s). HRMS(ESI) calcd for $\mathrm{C}_{22} \mathrm{H}_{21} \mathrm{O}_{3}{ }^{+}(\mathrm{M}+\mathrm{H})$ 333.1491, found 333.1490 .

\section{Tert-Butyl 2-(mesitylethynyl)-1-oxo-2,3-dihydro-1H-indene-2-carboxylate (7c)}

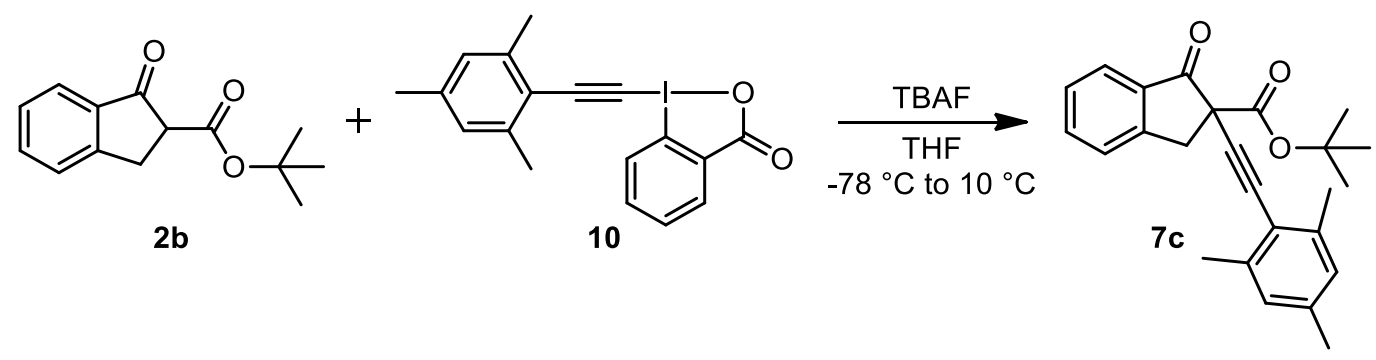

Following general procedure GP6, on a $0.08 \mathrm{mmol}$ scale using $\mathbf{2 b}(18.8 \mathrm{mg}, 0.0807 \mathrm{mmol}$, 1.0 equiv) and Mes-EBX (10) (40.9 $\mathrm{mg}, 0.105 \mathrm{mmol}, 1.30$ equiv). The reaction was finished after $20 \mathrm{~h}$ at $10{ }^{\circ} \mathrm{C}$. Purification by flash chromatography (Hexane/EtOAc 20:1) afforded 7c (25.7 $\mathrm{mg}, 0.0686 \mathrm{mmol}, 85 \%)$ as orange oil. 
$\mathrm{R}_{f} 0.71$ (PET/EtOAc 4:1, UV/Anisaldehyde). ${ }^{1} \mathrm{H}$ NMR (400 MHz, $\left.\mathrm{CDCl}_{3}\right) \delta 7.84(\mathrm{~d}, J=$ $7.7 \mathrm{~Hz}, 1 \mathrm{H} ; \operatorname{Ar} H), 7.65(\mathrm{~m}, 1 \mathrm{H} ; \operatorname{Ar} H), 7.51(\mathrm{~d}, J=7.6 \mathrm{~Hz}, 1 \mathrm{H} ; \operatorname{Ar} H), 7.43(\mathrm{t}, J=7.6 \mathrm{~Hz}, 1$ $\mathrm{H} ; \mathrm{Ar} H$ ), 6.81 (s, $2 \mathrm{H} ; \mathrm{Ar} H$ ), 3.97 (d, $\left.J=17.0 \mathrm{~Hz}, 1 \mathrm{H} ; \mathrm{CH}_{2}\right), 3.55\left(\mathrm{~d}, J=17.0 \mathrm{~Hz}, 1 \mathrm{H} ; \mathrm{CH}_{2}\right.$ ), 2.36 (s, $6 \mathrm{H}$; $\left.\left(\mathrm{CH}_{3}\right)_{2}\right), 2.25$ (s, $3 \mathrm{H}$; $\left.\mathrm{CH}_{3}\right), 1.48$ (s, $9 \mathrm{H}$; OC( $\left.\left.\mathrm{CH}_{3}\right)_{3}\right) .{ }^{13} \mathrm{C}$ NMR $(101 \mathrm{MHz}$, $\left.\mathrm{CDCl}_{3}\right) \delta 197.0,167.3,152.2,140.5,137.6,135.6,133.6,128.0,127.4,126.3,125.6,119.4$, 93.3, 83.2, 81.5, 57.3, 41.3, 29.7, 27.8, 21.3, 20.9. IR $\vee 3307$ (w), 3078 (w), 2980 (w), 2926 (w), $2857(\mathrm{w}), 2253(\mathrm{w}), 2146(\mathrm{w}), 1724(\mathrm{~m}), 1610(\mathrm{w}), 1478(\mathrm{w}), 1465(\mathrm{w}), 1394(\mathrm{w}), 1370$ (w), $1294(w), 1255(\mathrm{~m}), 1212(\mathrm{w}), 1152(\mathrm{~s}), 1036$ (w), 994 (w), 908 (s), $845(\mathrm{w}), 810(\mathrm{w})$, $732(\mathrm{~s}), 646(\mathrm{~m}), 633(\mathrm{~s})$. HRMS(ESI) calcd for $\mathrm{C}_{25} \mathrm{H}_{26} \mathrm{O}_{3} \mathrm{Ag}^{+}\left(\mathrm{M}+\mathrm{Ag}^{106.9}\right) 481.0933$, found 481.0945; calcd for $\mathrm{C}_{25} \mathrm{H}_{26} \mathrm{O}_{3} \mathrm{Ag}^{+}\left(\mathrm{M}+\mathrm{Ag}^{108.9}\right)$ 483.0933, found 483.0933.

\section{Spectra of New Compounds}


solvent: $<\mathrm{CDCl} 3$

$\mathrm{TMS}=-1$

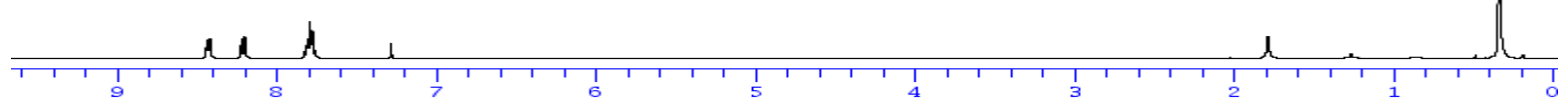

Solvent: $<C D C I 3>$
Frequency. 100.612769M Hz
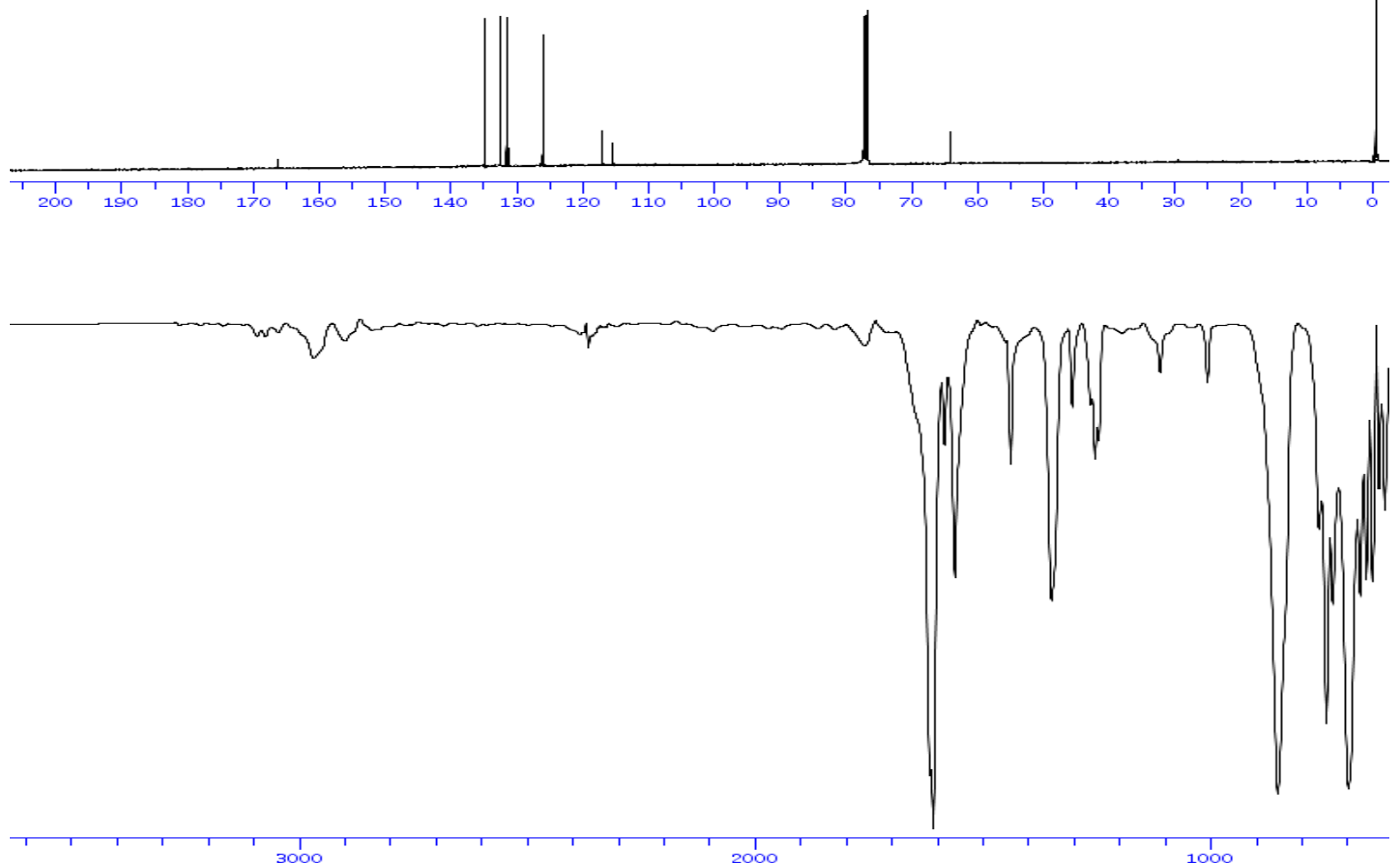


\section{DSC of TMS-EBX}

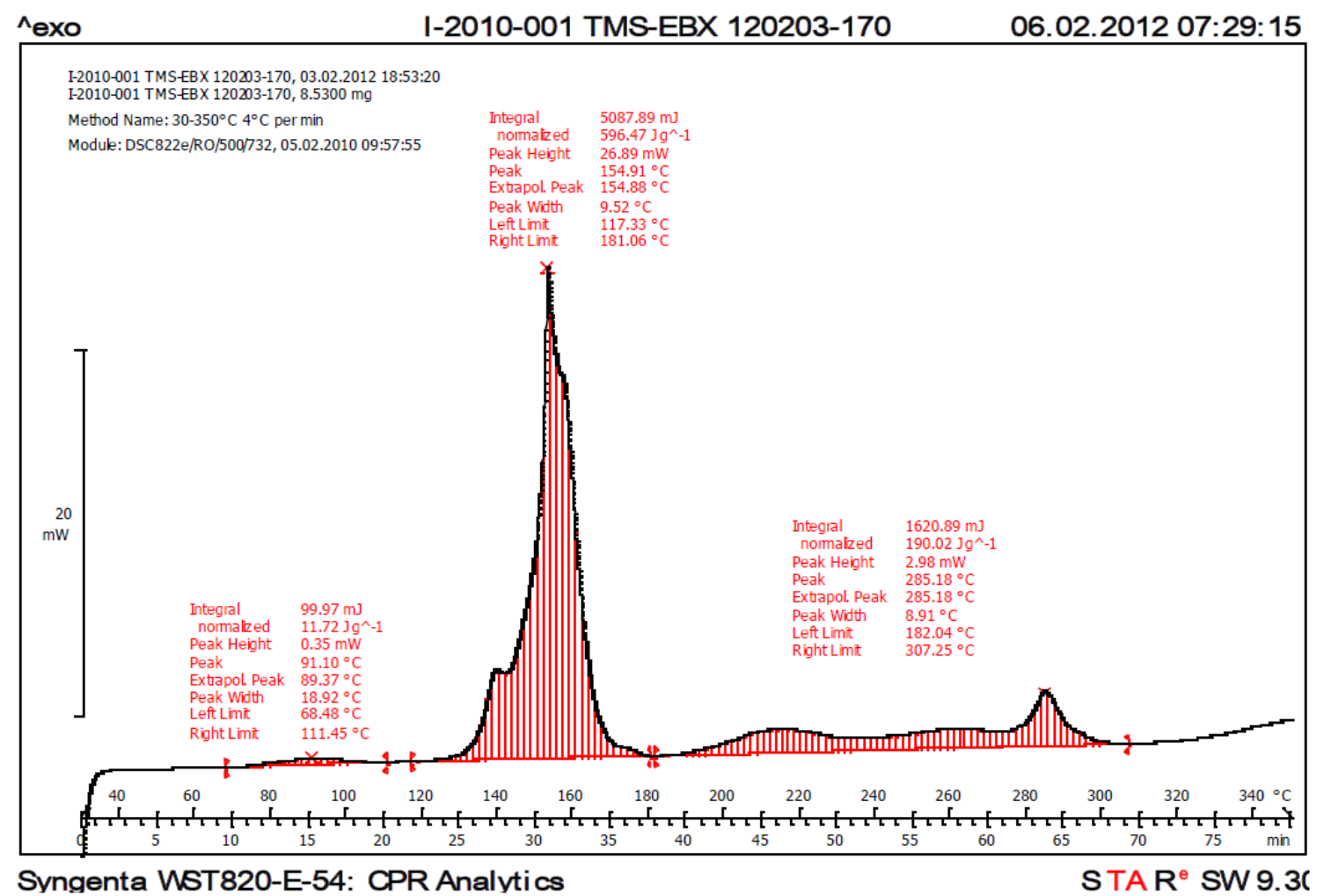

Performed by R. Mondière for J. Cassayre on $6^{\text {th }}$ February 2012. 
solvent: $\angle \mathrm{CDCl} 3>$
Frequency. $400.13 \mathrm{MHz}$<smiles>O=C1OI(C#CSC(F)F)c2ccccc21</smiles>

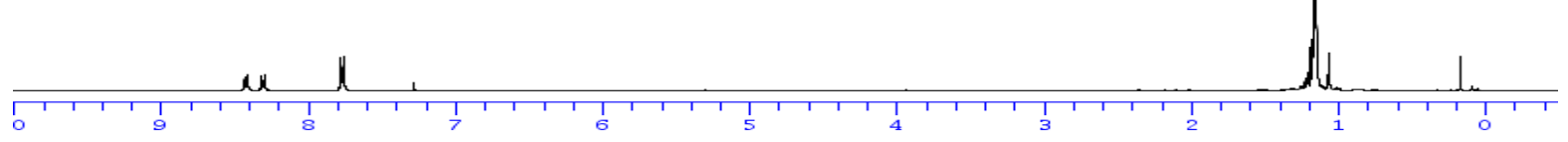

solvent: $<\mathrm{CDCI}>$
Frequency. $100.61 \mathrm{MHz}$
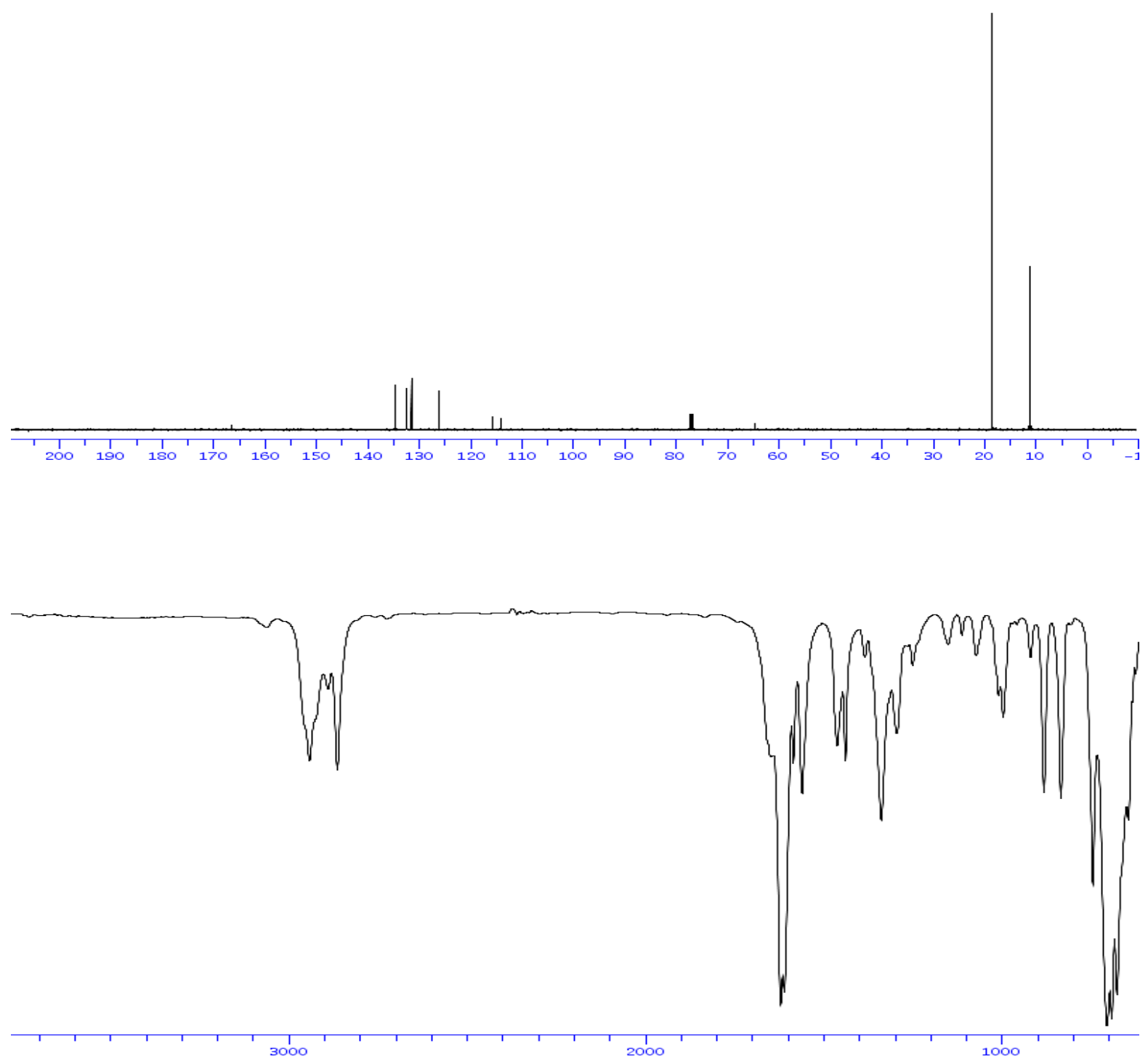


\section{DSC of TIPS-EBX}

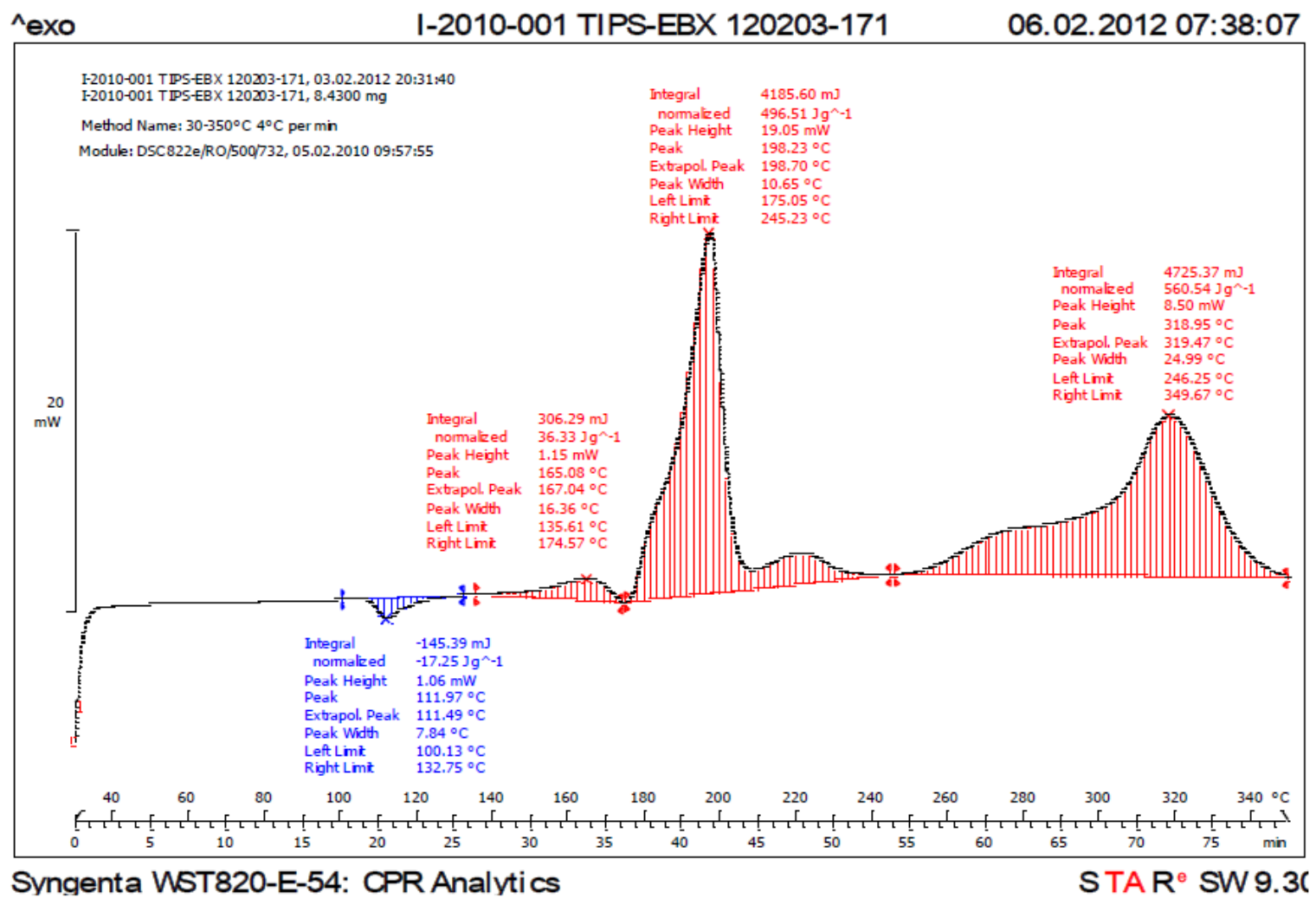

Performed by R. Mondière for J. Cassayre on $6^{\text {th }}$ February 2012. 
5-Nitro-1-[(Triisopropylsilyl)ethynyl]-1,2-benziodoxol-3(1H)-one (1c)

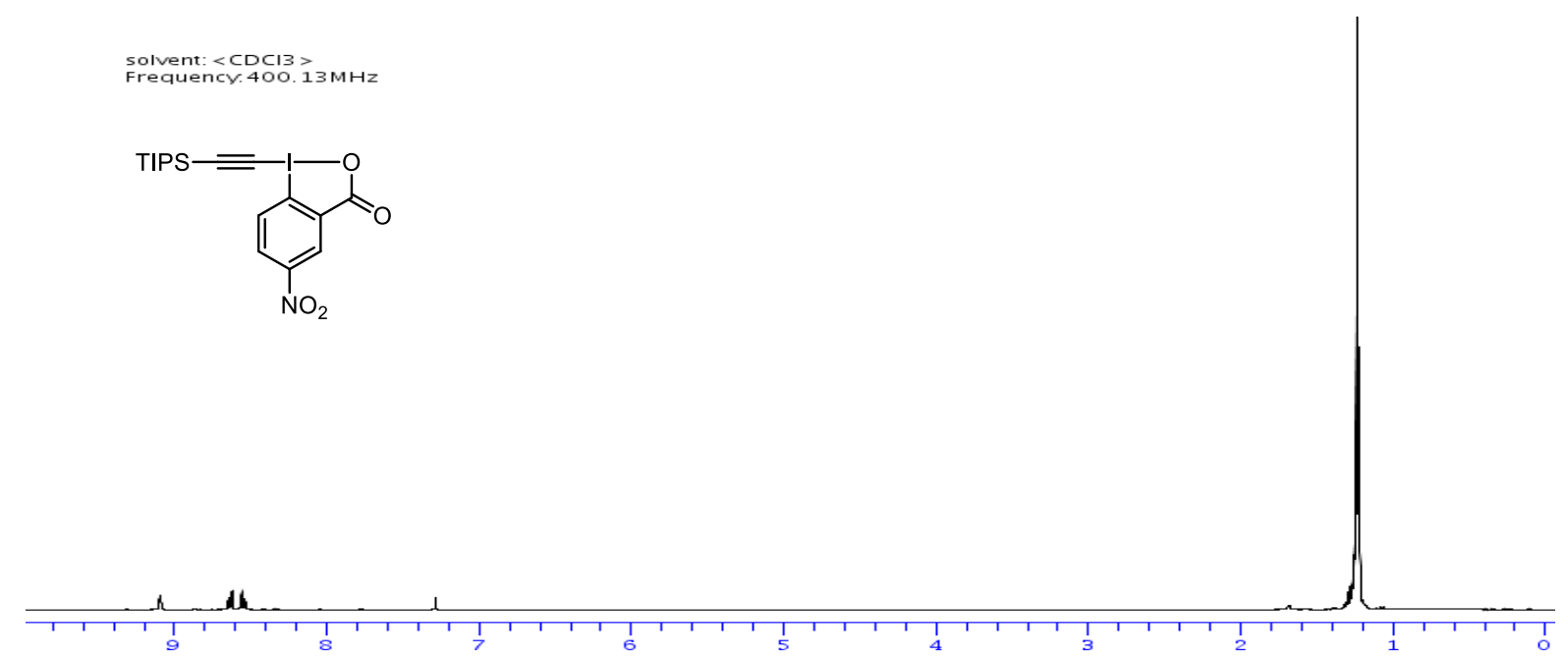

solvent: $<\mathrm{CDCl} 3$

Frequency. $100.612769 \mathrm{MHz}$
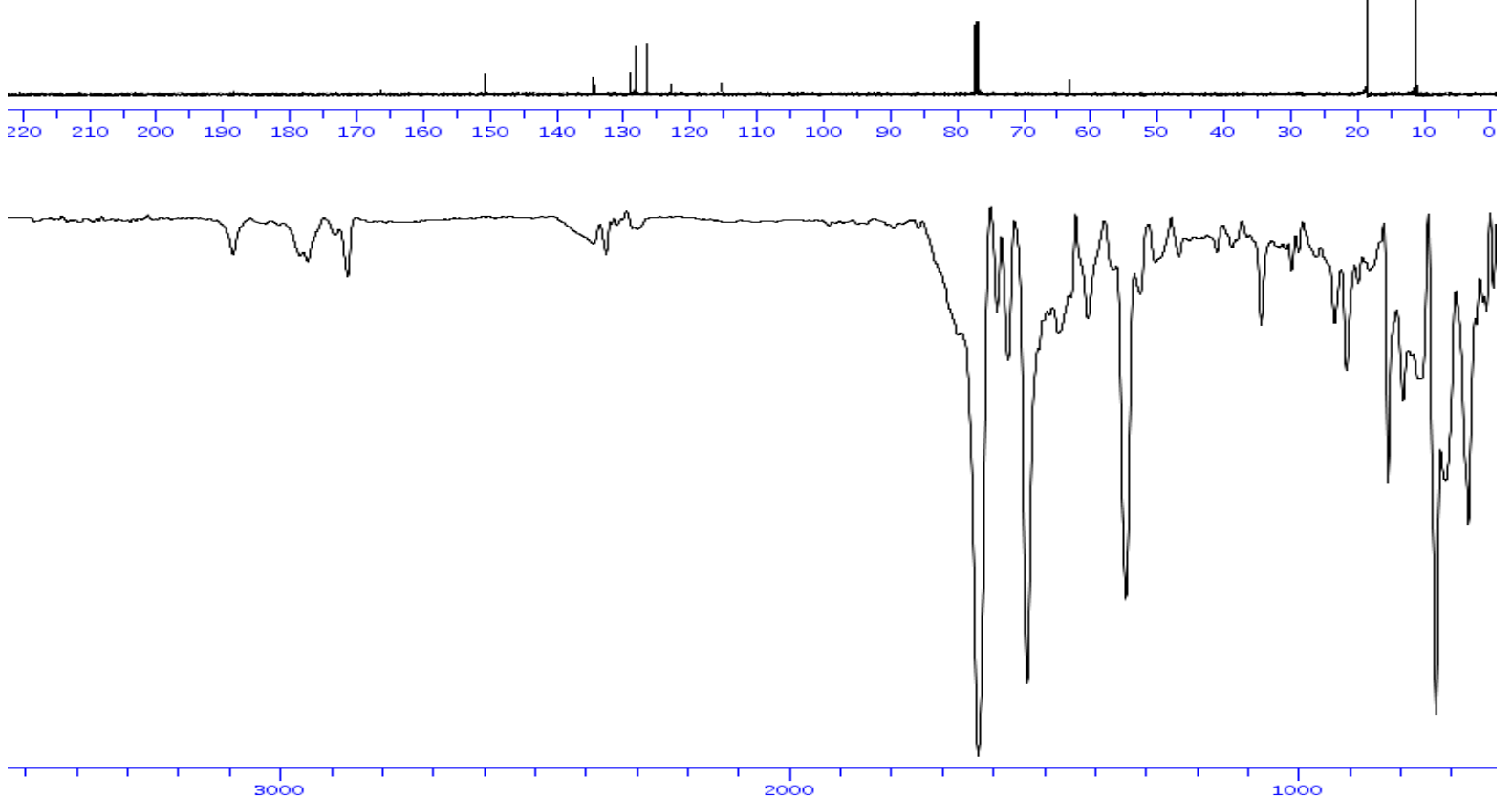
Solvent: $<\mathrm{CDCI} 3>$
Frequency. $400.13 \mathrm{MHz}$<smiles>COc1cc2c(cc1OC)I(C#CSC(F)F)OC2=O</smiles>

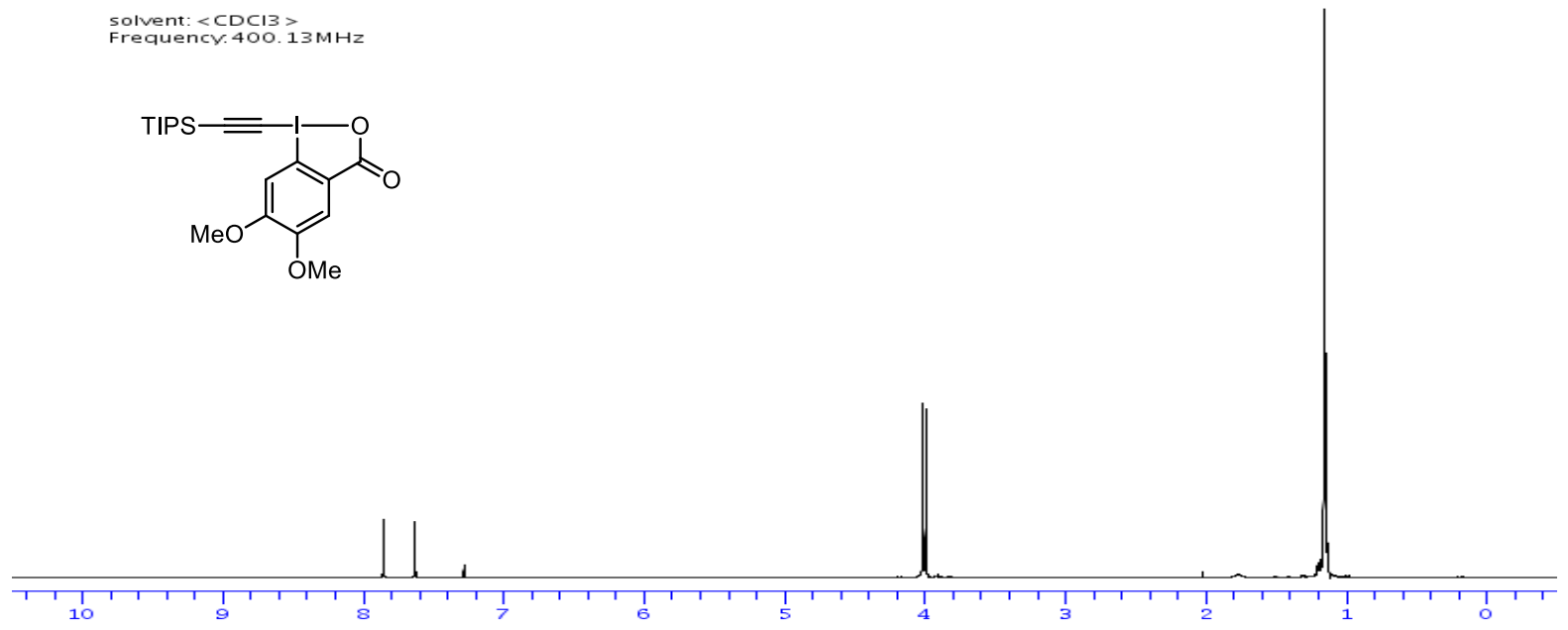

Solvent: $<\mathrm{CDCl} 3>$ $>$ Frequency $100.612769 \mathrm{MHz}$

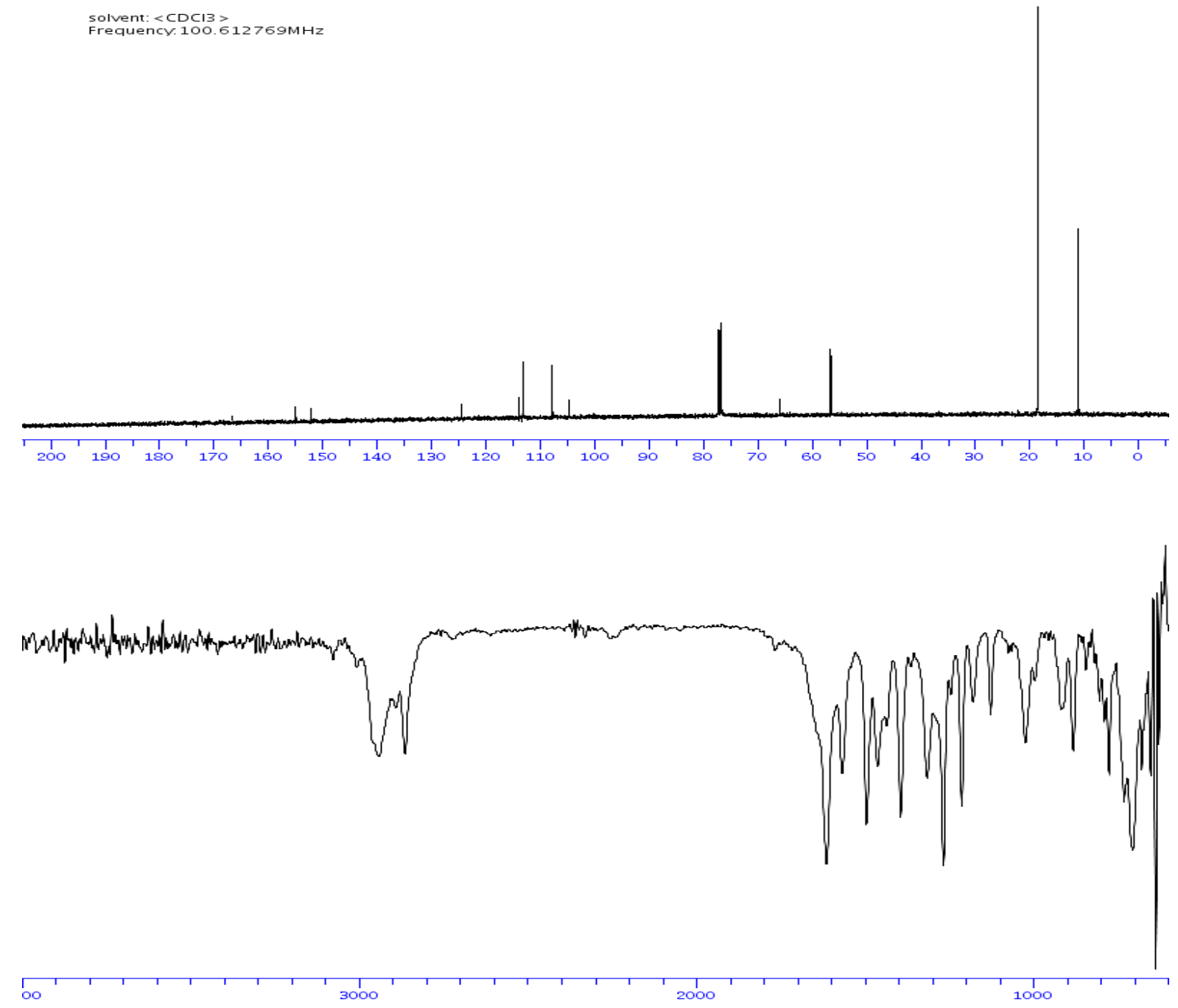


solvent: $<\mathrm{CDCl}>>$
Frequency. $400.13 \mathrm{MH}$<smiles>Cc1cccc2c1I(C#CSC(C)C)OC2=O</smiles>

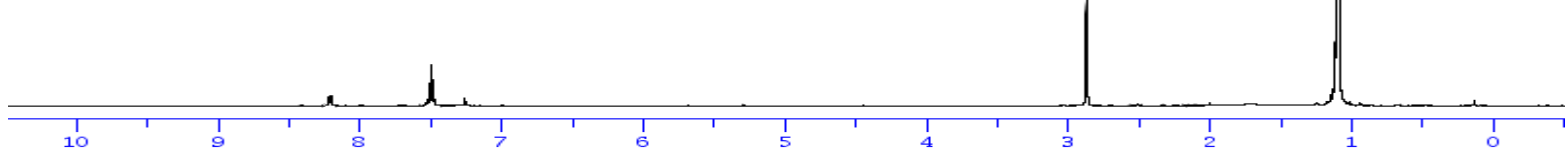

Solvent: $\angle \mathrm{CDCl} 3\rangle$

Frequency.100.612769M Hz
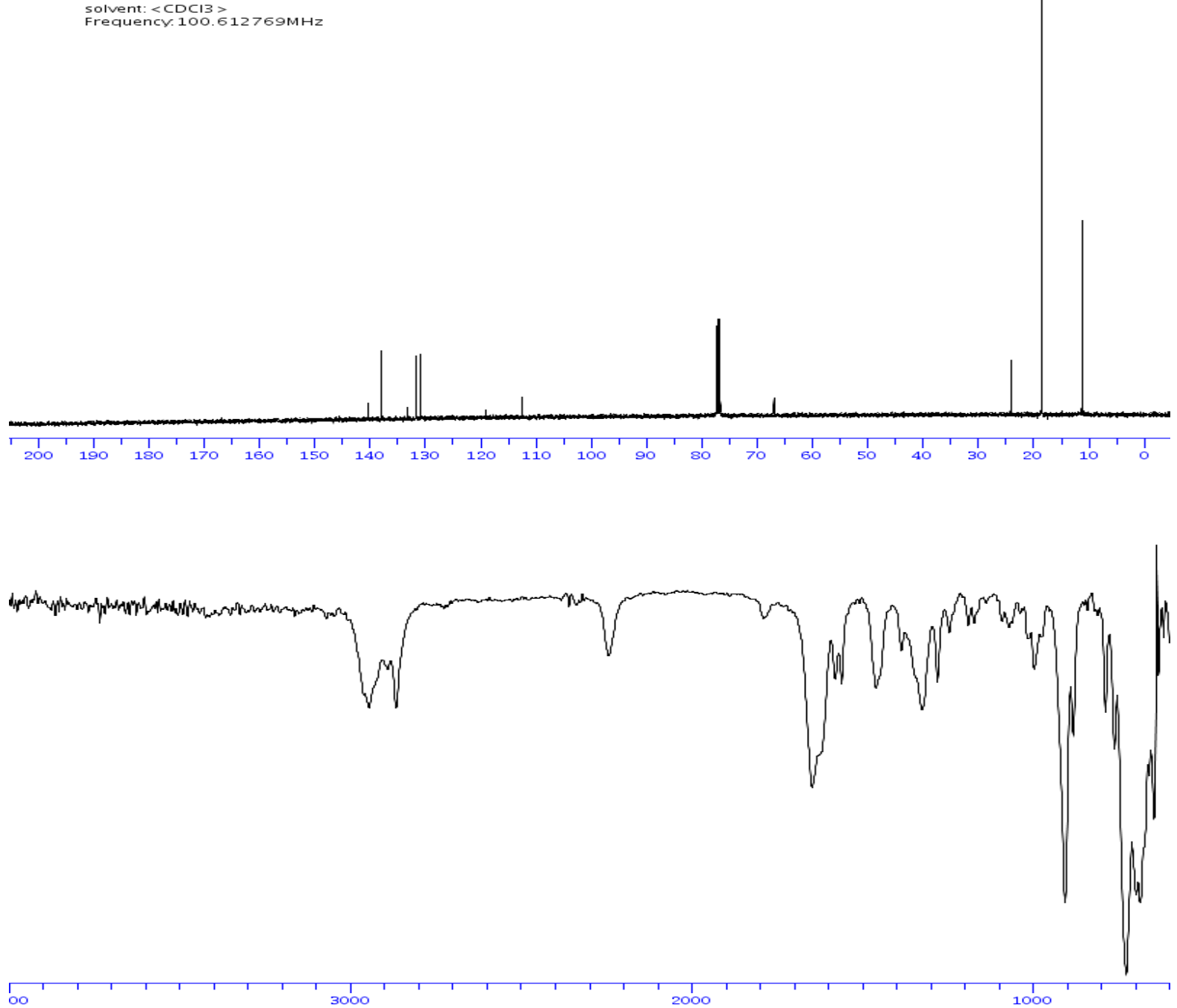
solvent: $<\mathrm{CDCl} 3>$
Frequency. $400.13 \mathrm{MHz}$

TIPS -<smiles>Cc1cccc2c1C(=O)OI2I</smiles>

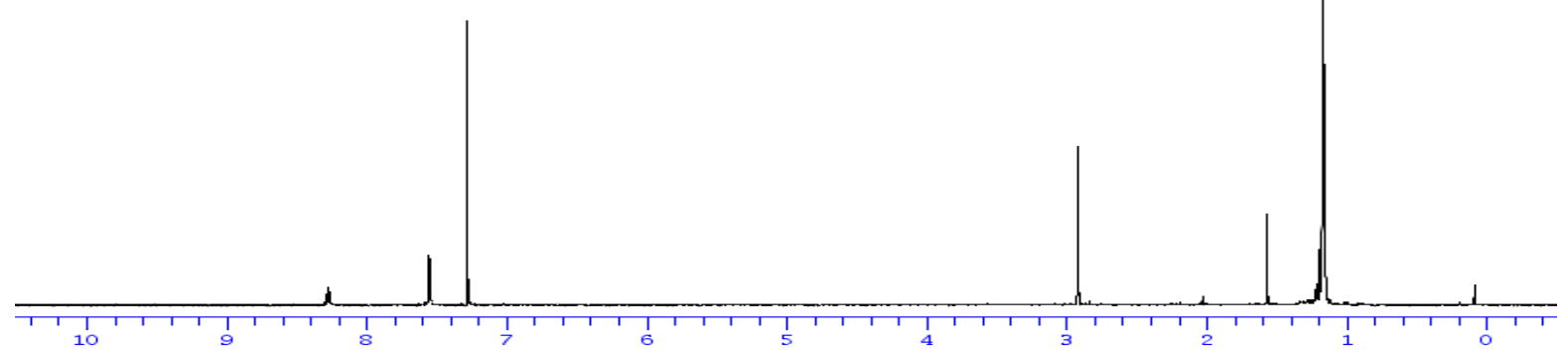

Solvent: $<$ CDCI3 $>$
Frequency.100.612769MHz
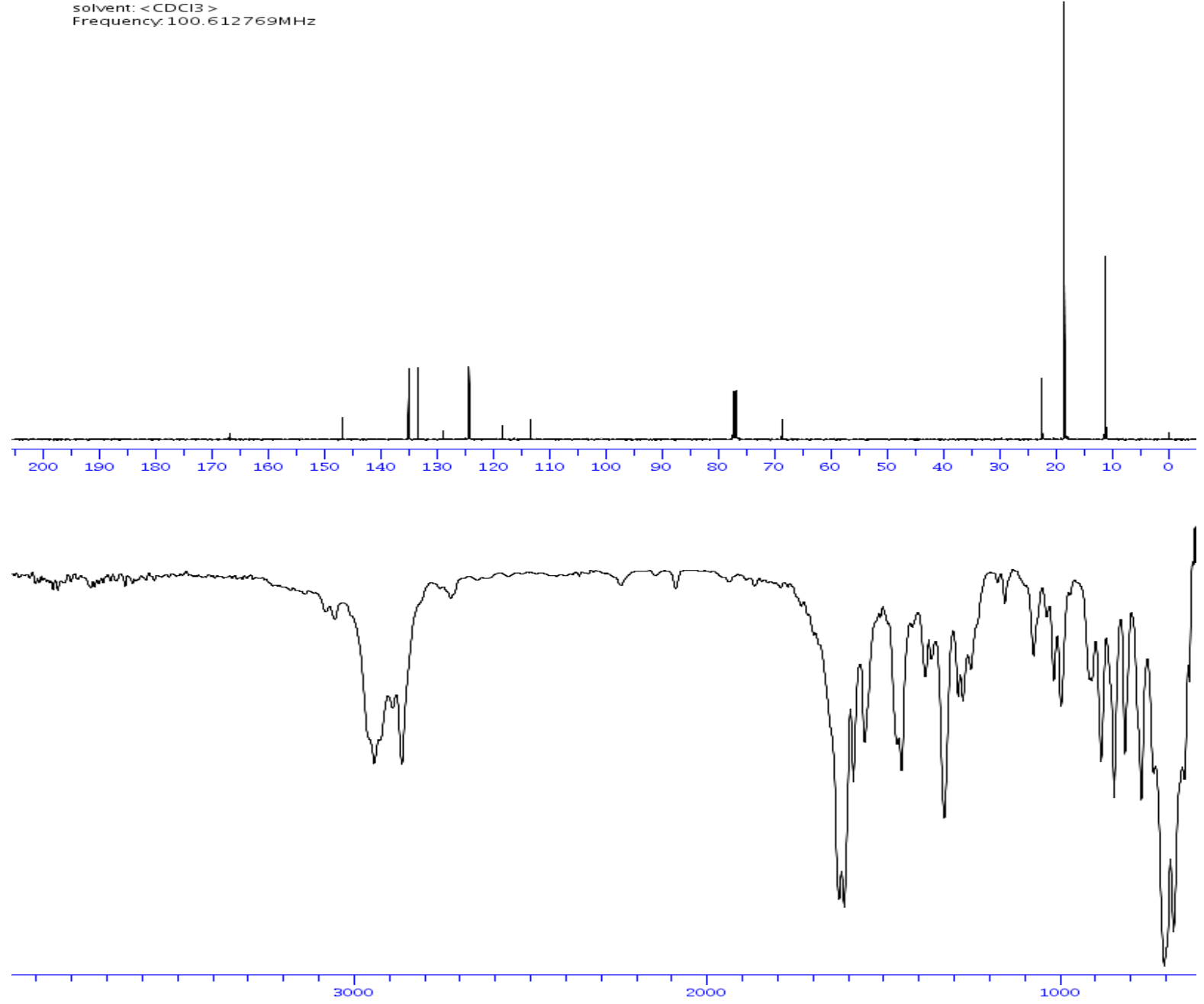
Solvent: $<\mathrm{CDCI}>>$
Frequency. $400.13 \mathrm{MHz}$<smiles>CC1(C)OI(C#CS[In])c2ccccc21</smiles><smiles>C#CC(=C)C(C)(C)C#CC</smiles>

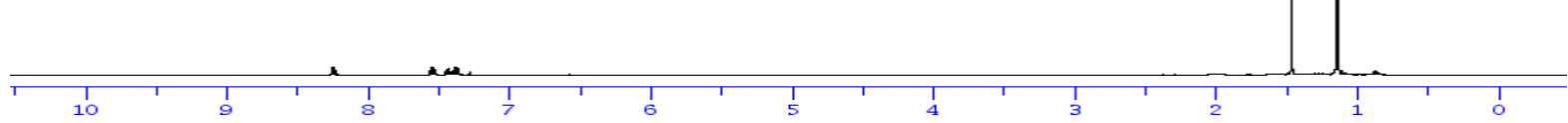

solvent: $<\mathrm{CDCl} 3>$
Frequency.100.612769MHz
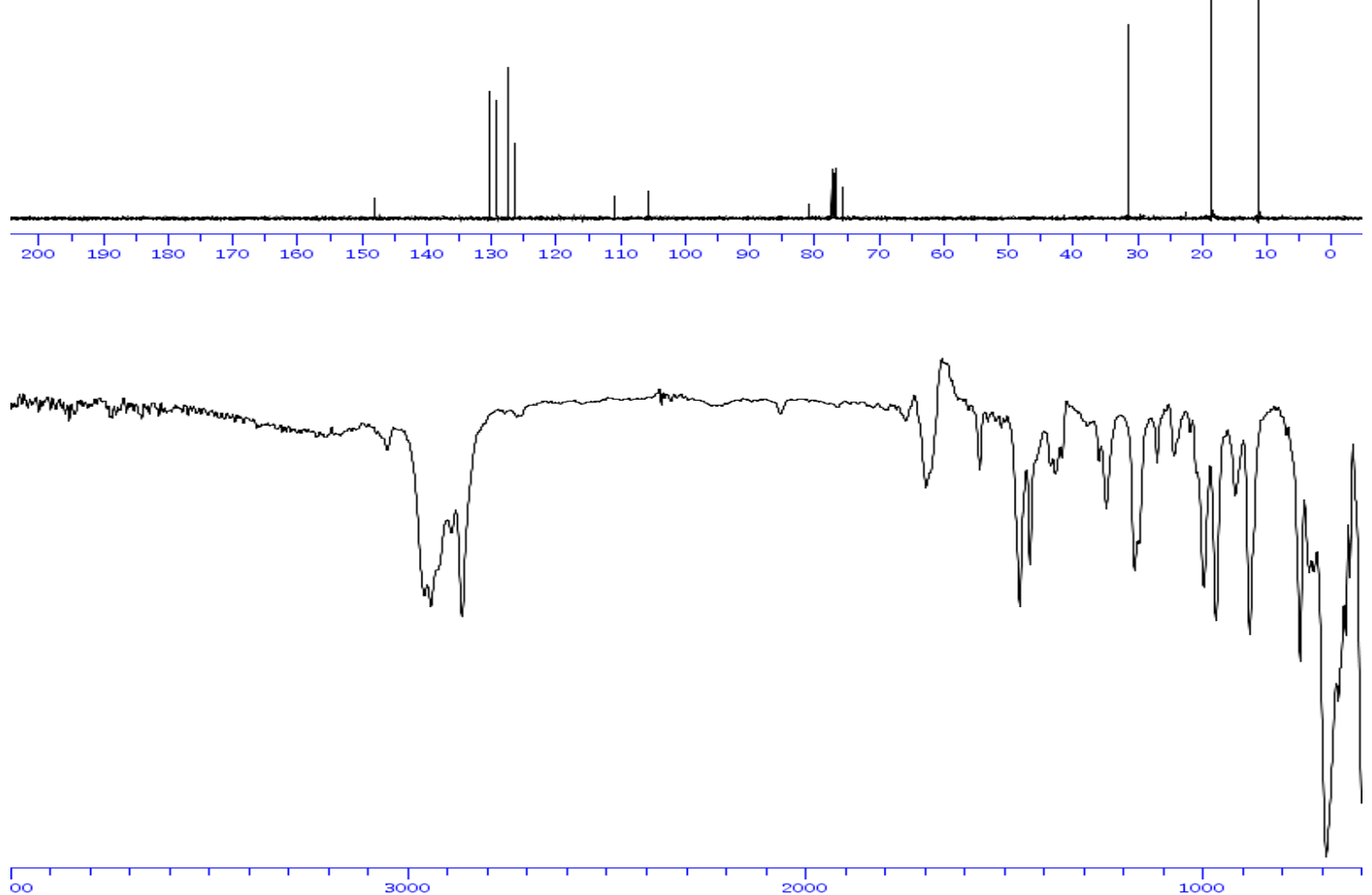
1-[(Triisopropylsilyl)ethynyl]-3,3-bis(trifluoromethyl)-3(1H)-1,2-benziodoxole (6)

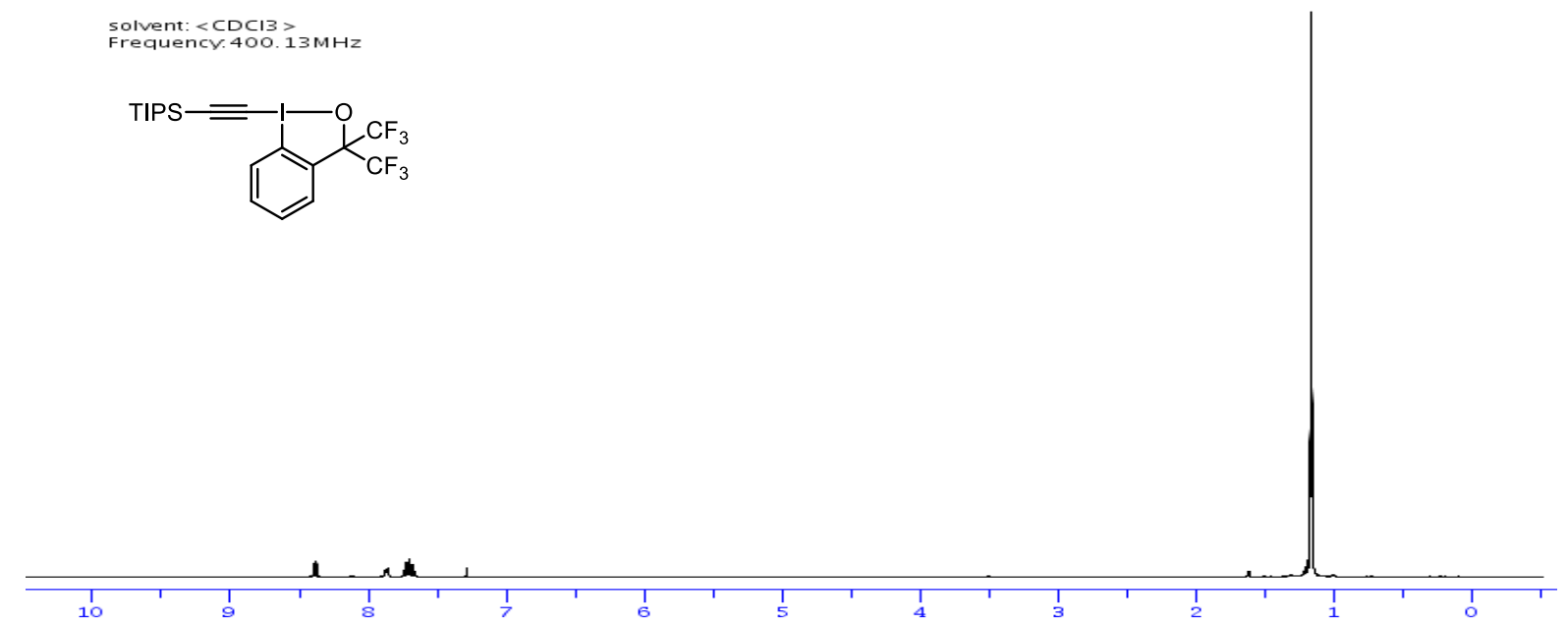

solvent: $<\mathrm{CDCl} 3>$
Frequency.100.612769M Hz
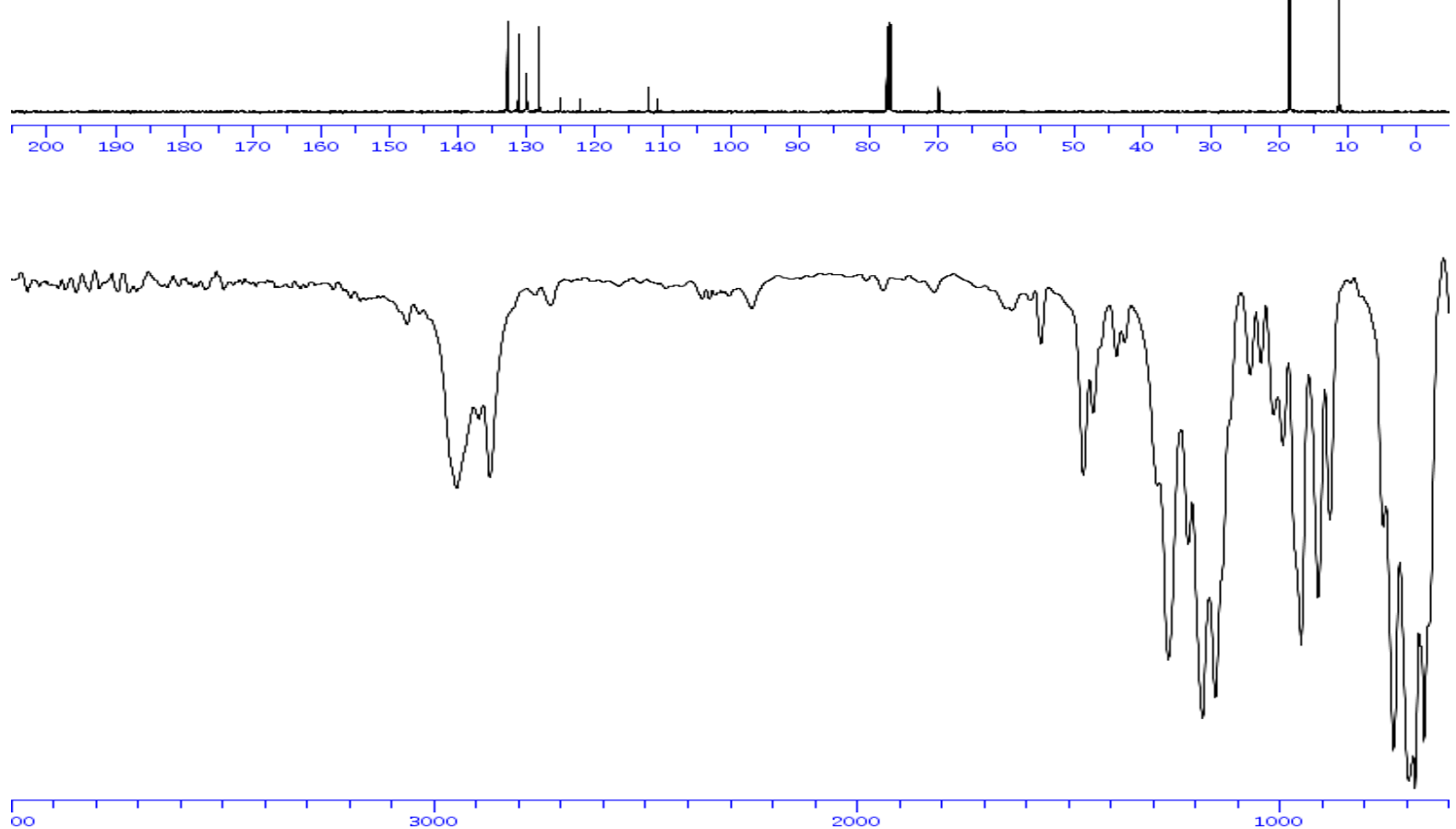
5-Nitro-1-[Phenylethynyl]-1,2-benziodoxol-3(1H)-one (8b)

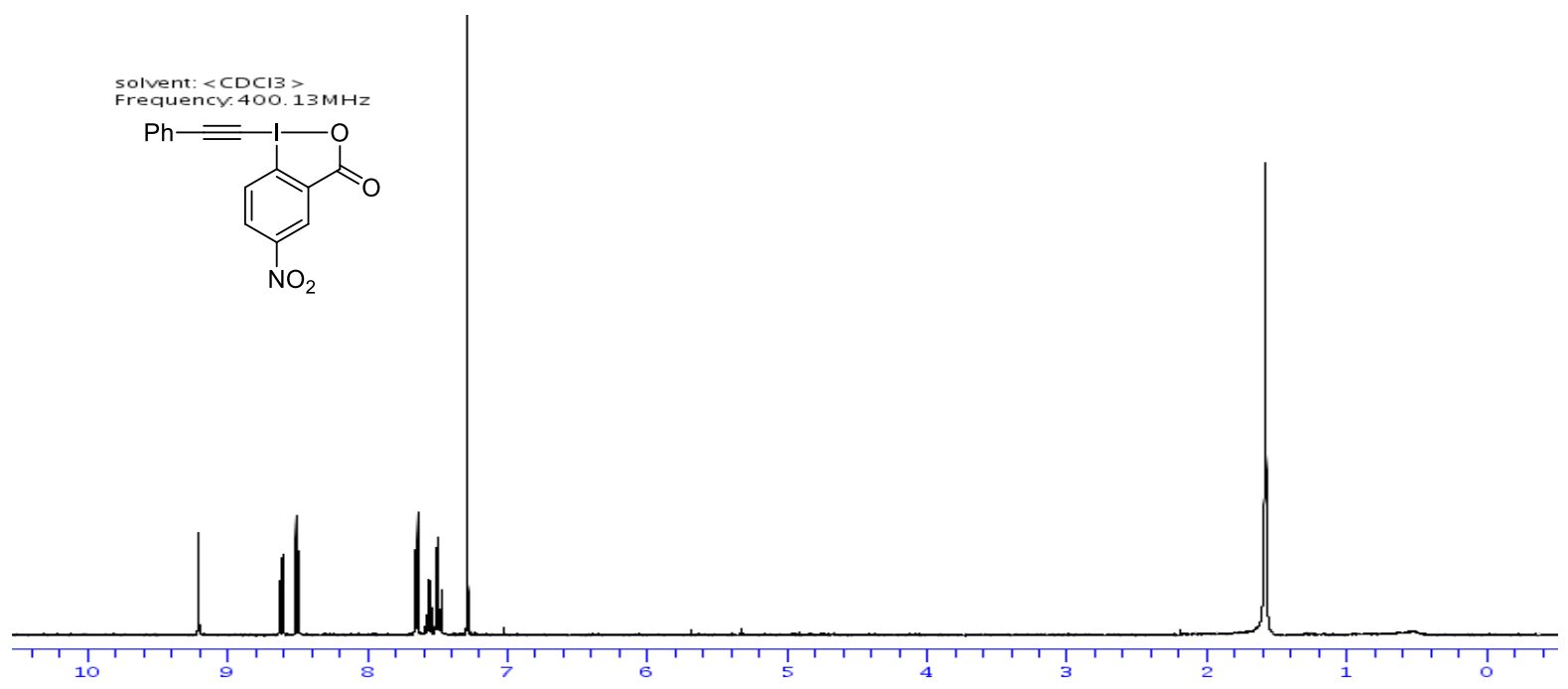

Solvent: $<\mathrm{CDCI}>$
FrequencY. $100.612769 \mathrm{MHz}$
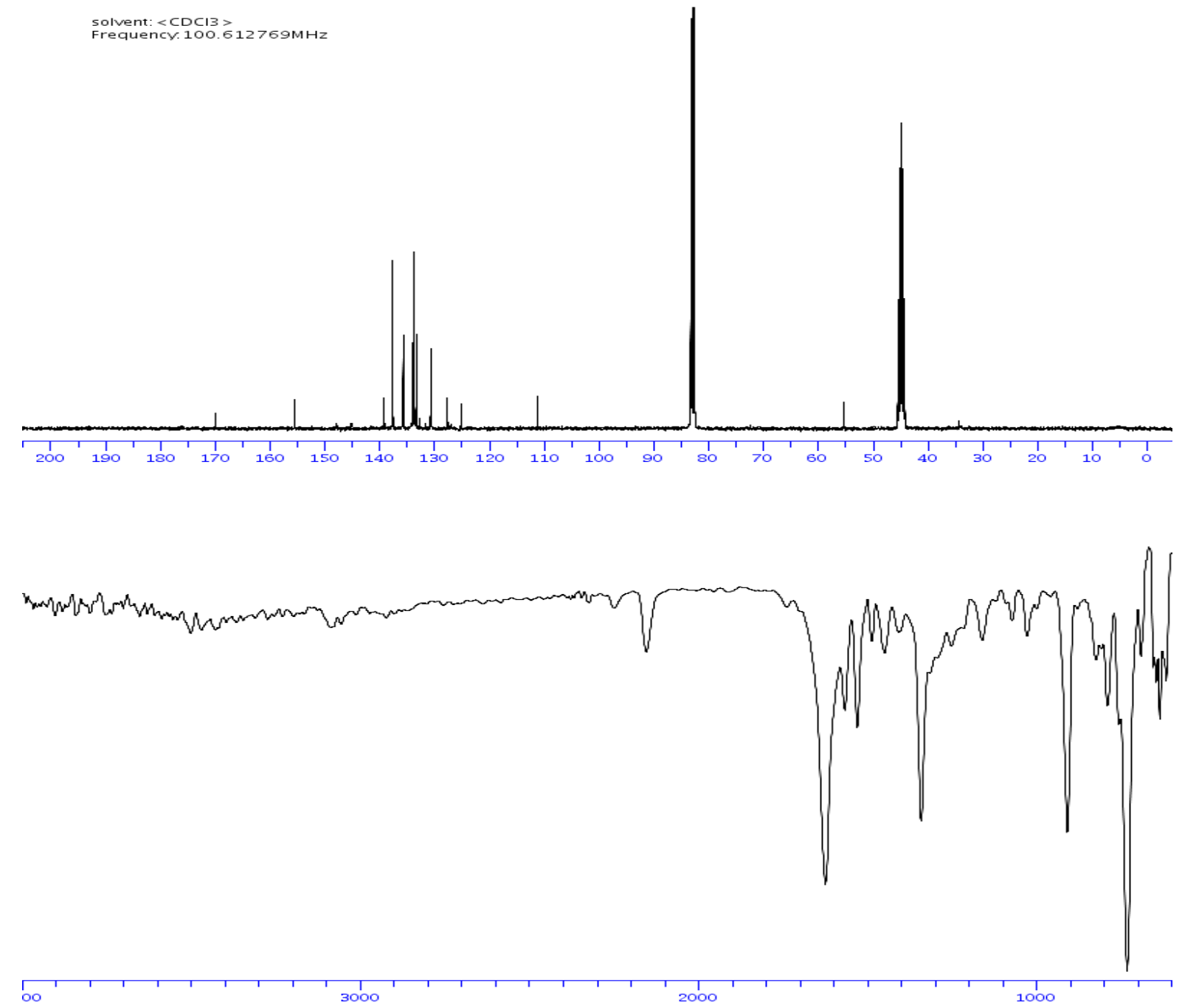
5-Fluoro-1-[Phenylethynyl]-1,2-benziodoxol-3(1H)-one (8c)
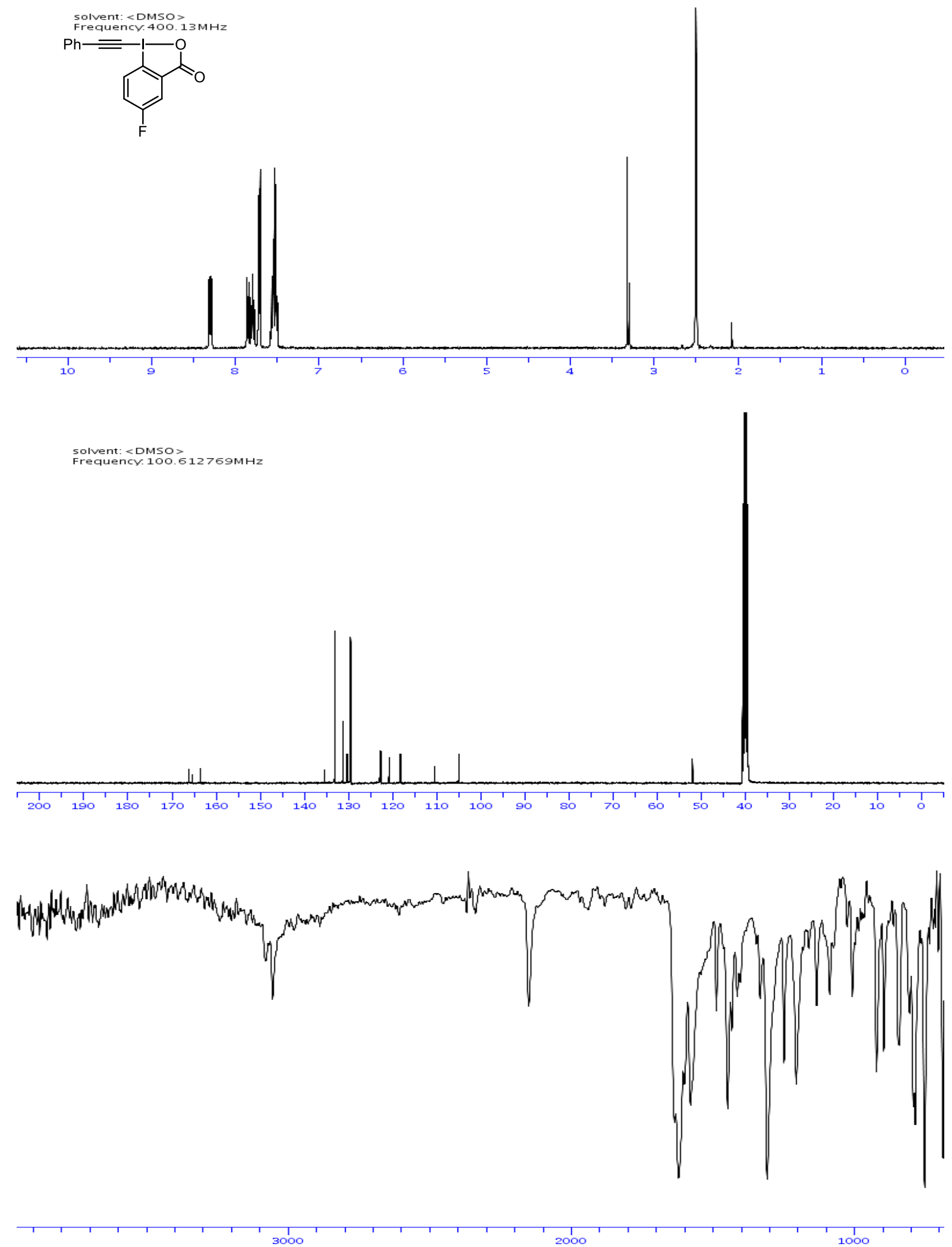
solvent: $<\mathrm{CDCI} 3$<smiles>COc1cc2c(cc1OC)I(C#Cc1ccccc1)OC2=O</smiles>

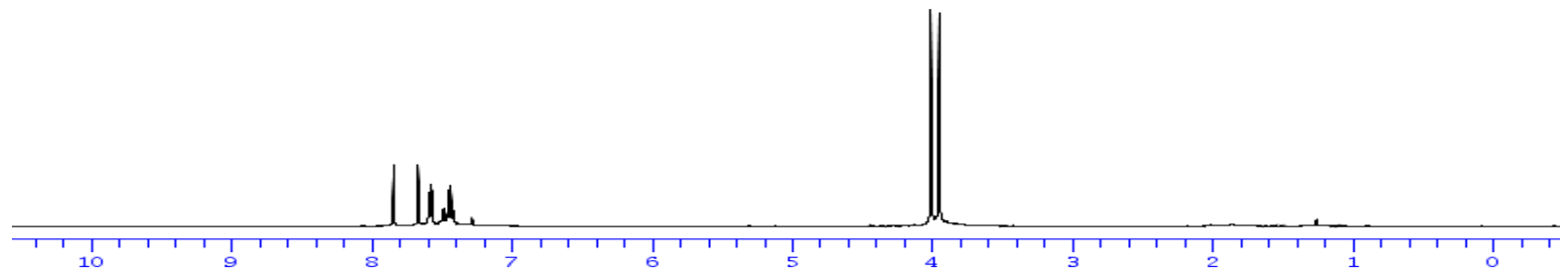

solvent: $\langle\mathrm{CDCl} 3\rangle$
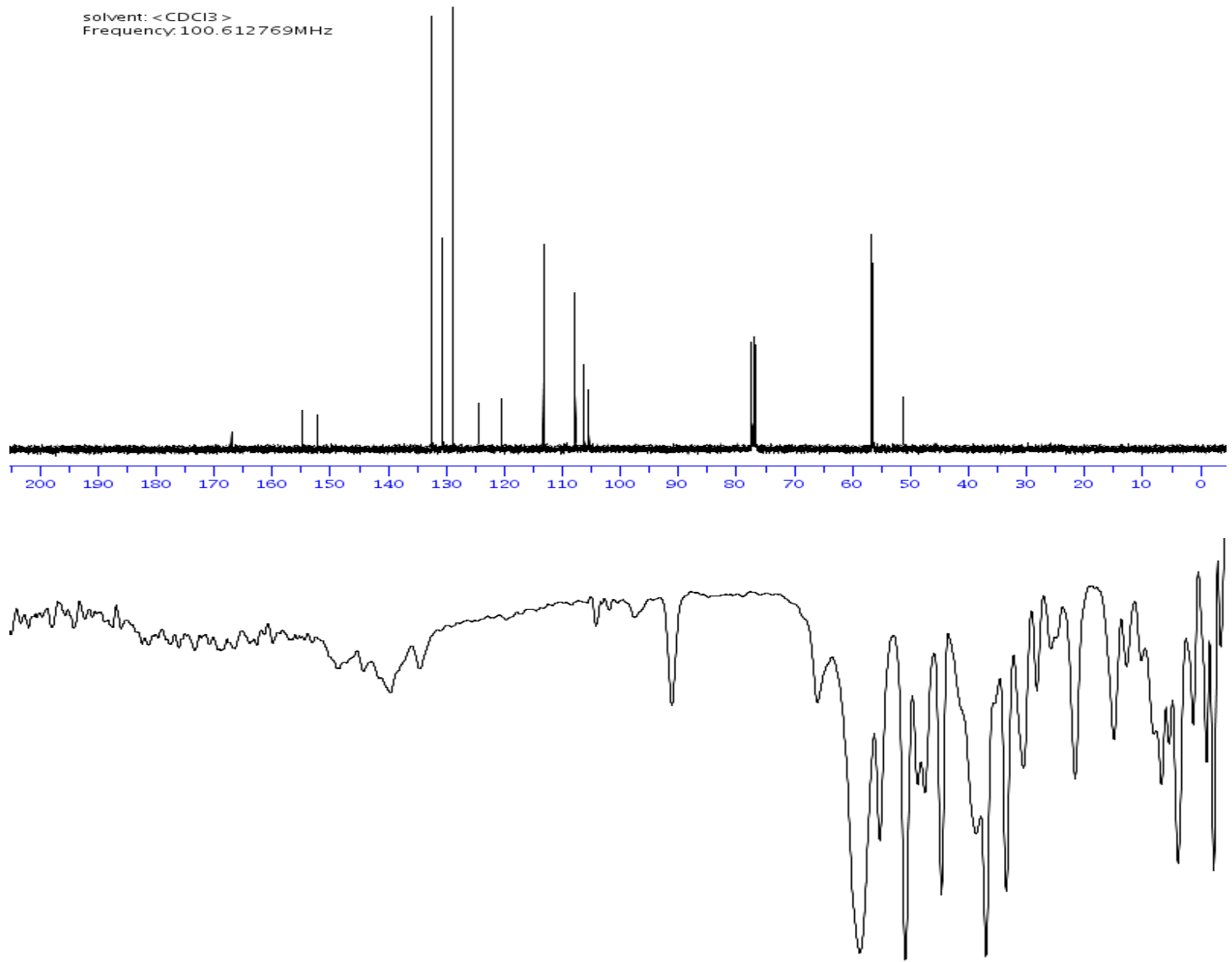

oo 3000 2000 1000 
Solvent: $<\mathrm{CDCI} 3>$
Frequency. $400.13 \mathrm{MHz}$<smiles>O=C1OI(C#Cc2ccc([N+](=O)[O-])cc2)c2ccccc21</smiles>

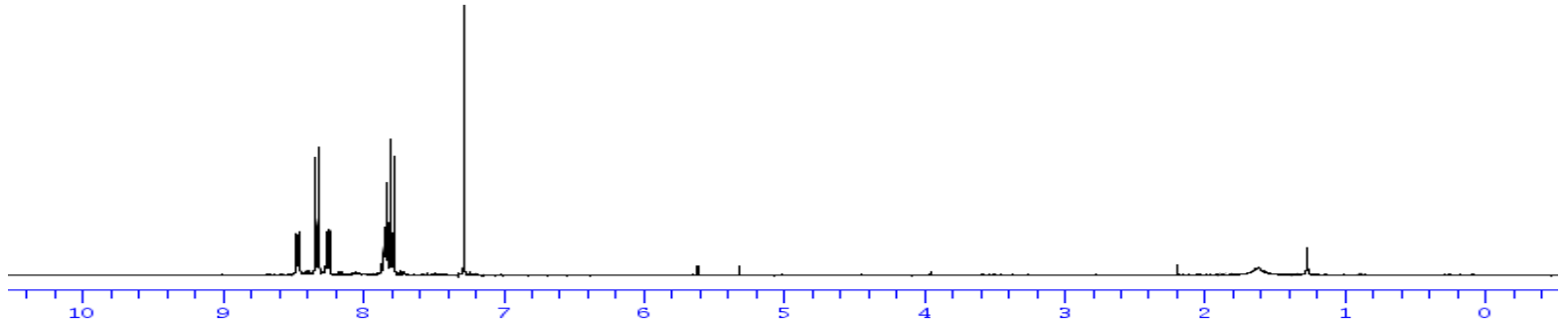

solvent: $<\mathrm{CDCl}>$
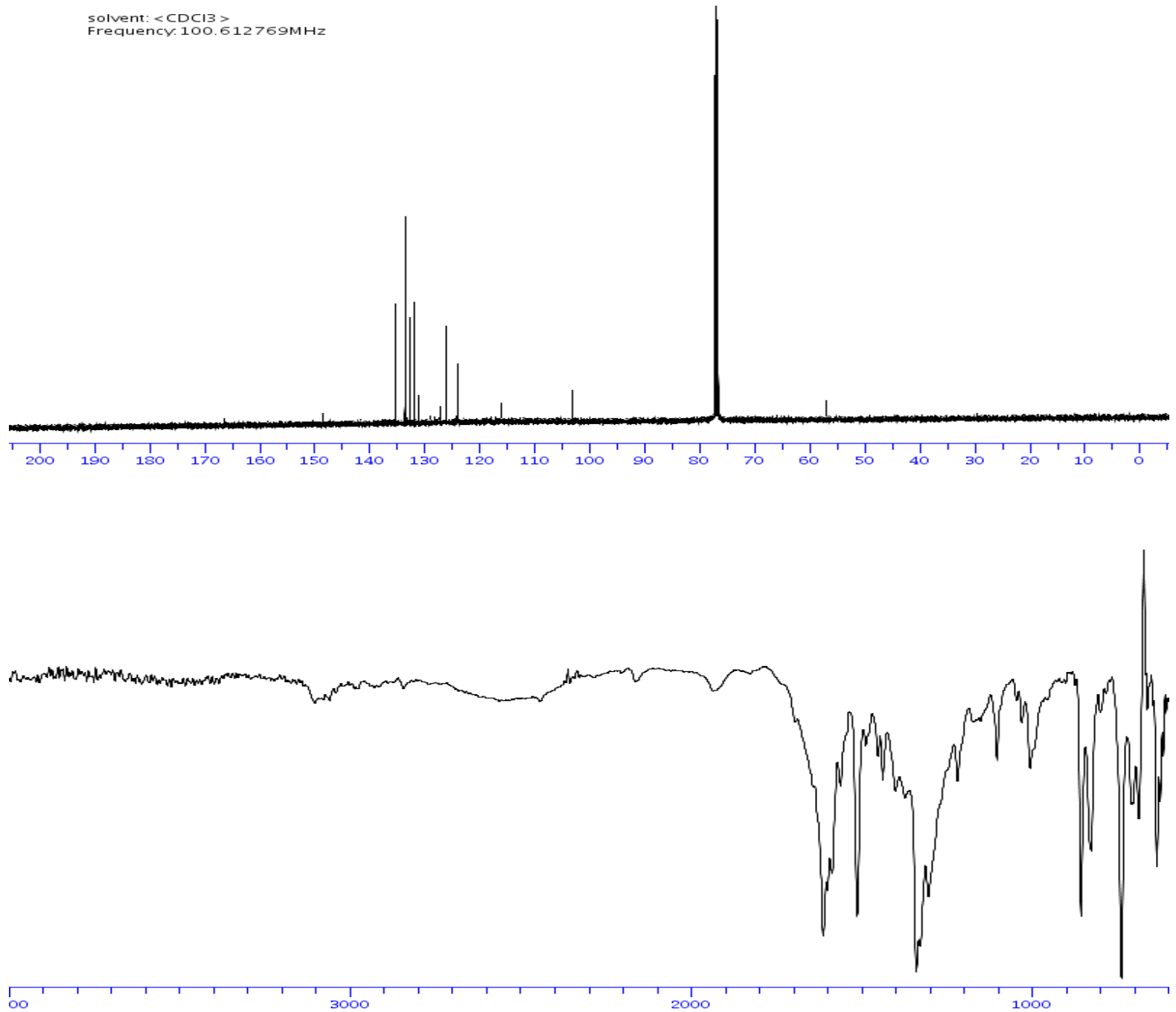

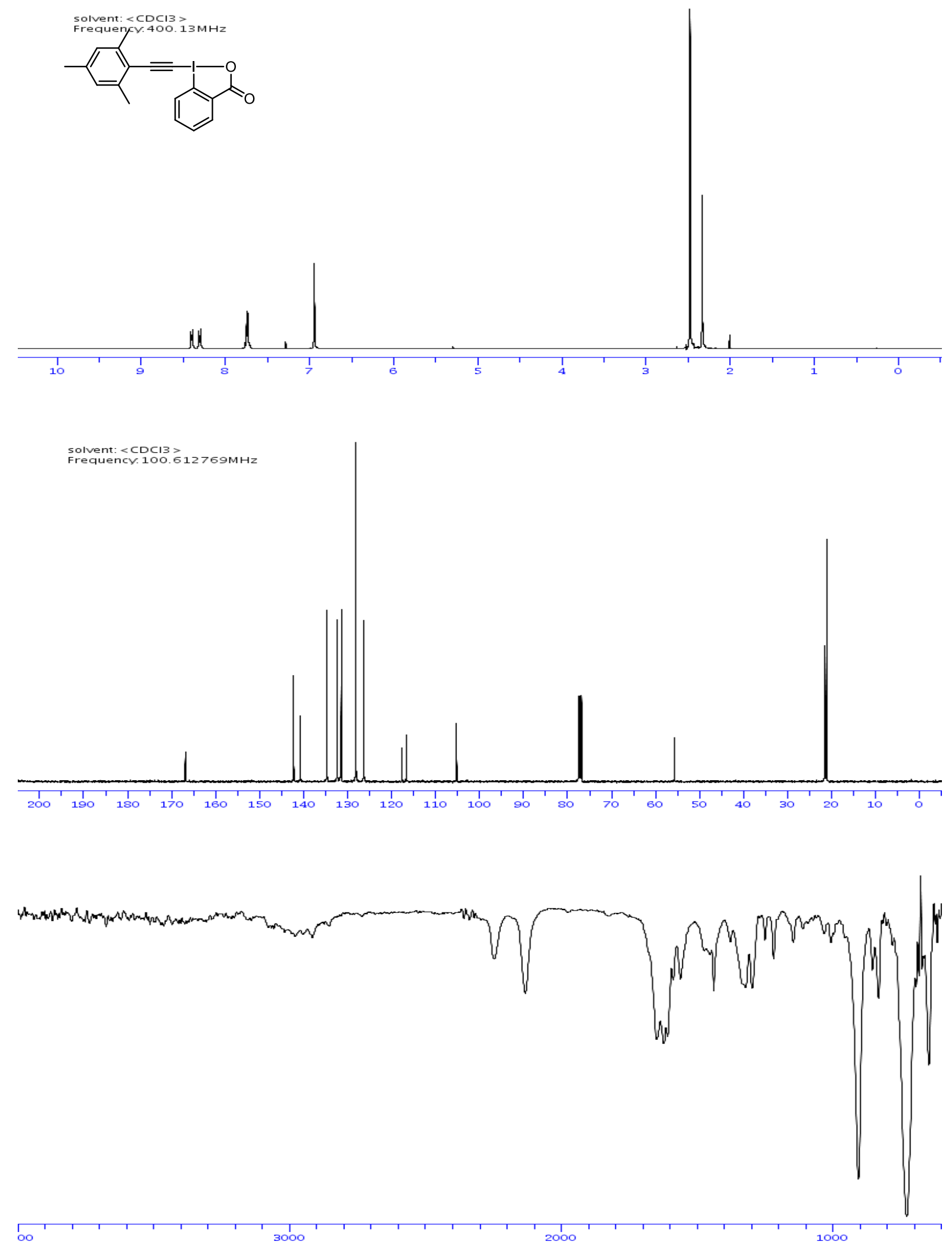

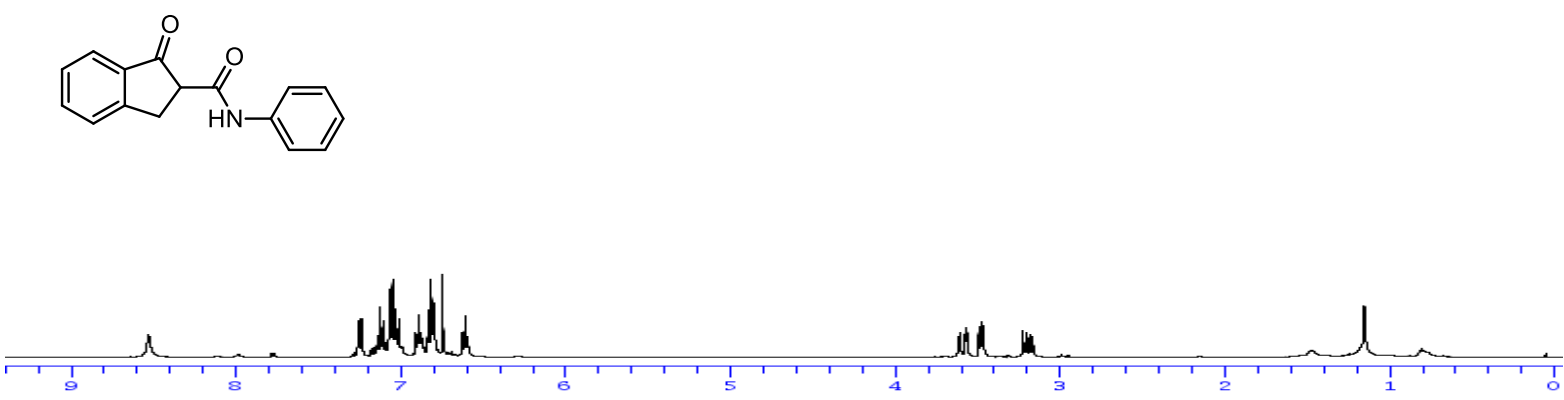

Solvent: $<C D C I 3>$
Frequency.100.612769MHz
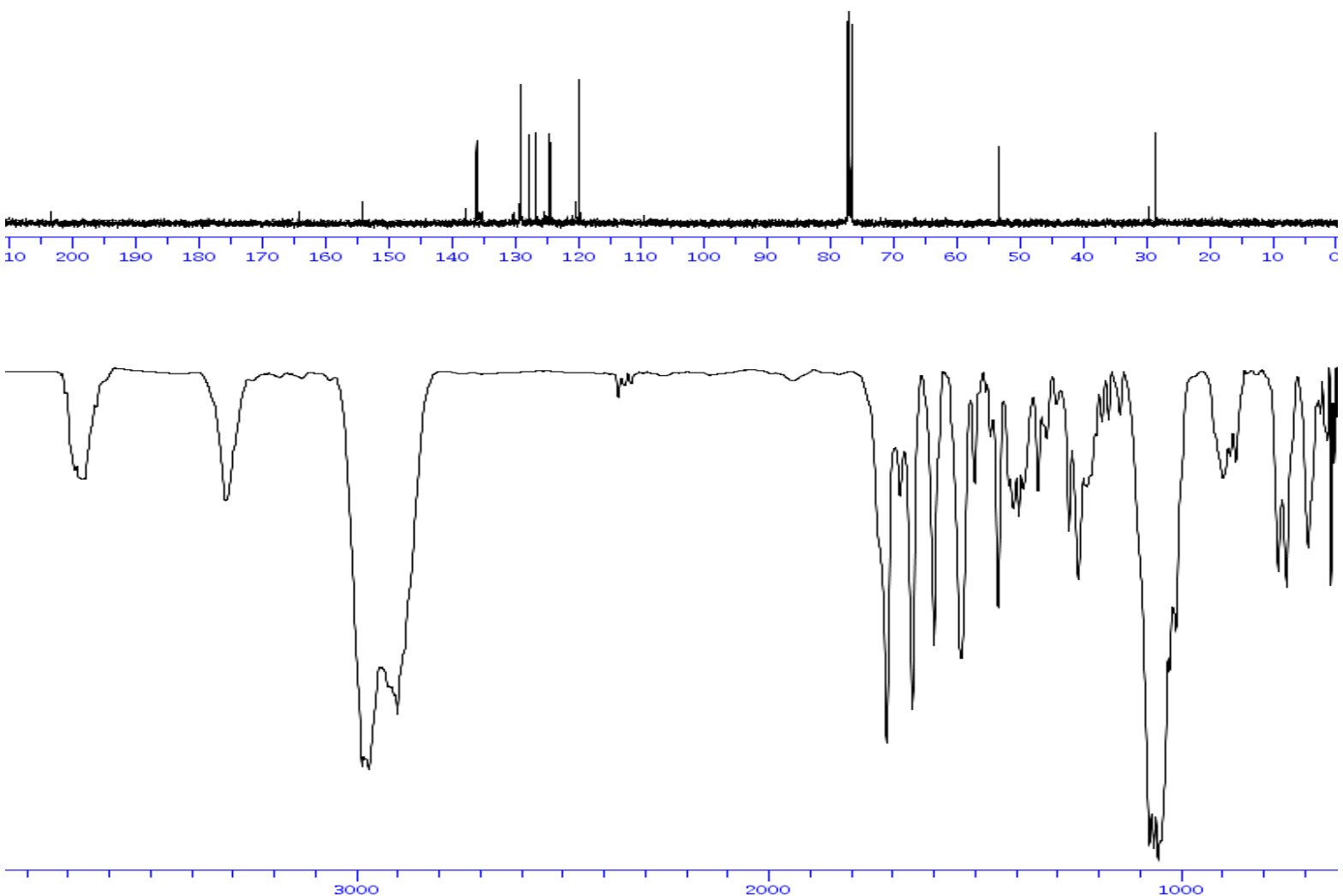

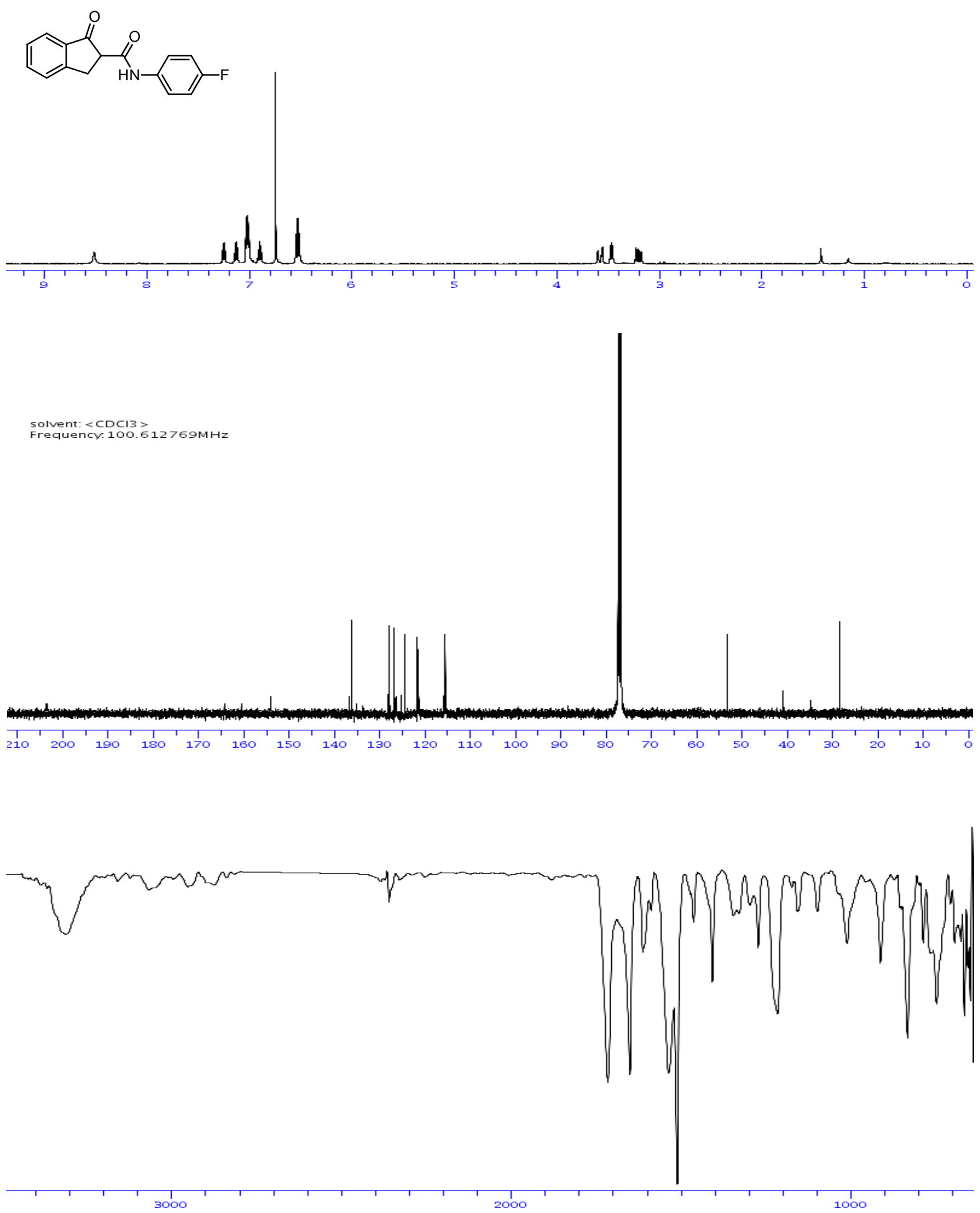
solvent: $\angle C D C l 3>$
Frequency $400.13 \mathrm{MHz}$<smiles>O=C(Nc1cc(C(F)(F)F)cc(C(F)(F)F)c1)C1Cc2ccccc2C1=O</smiles>

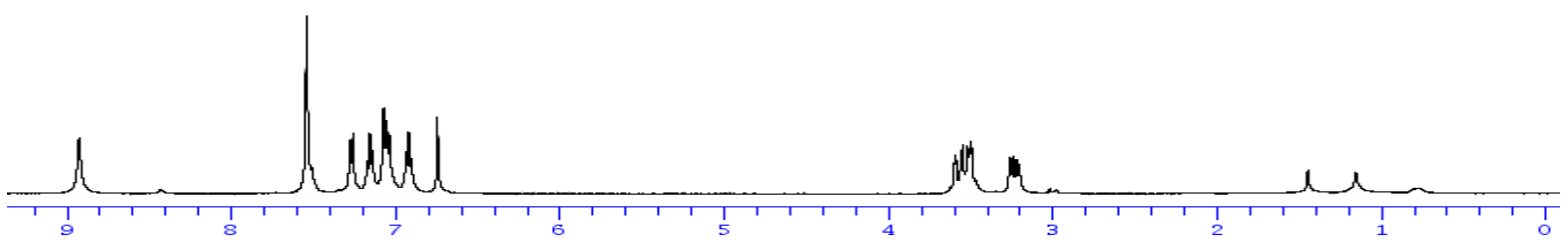

solvent: $\langle\mathrm{CDC1} 3\rangle$
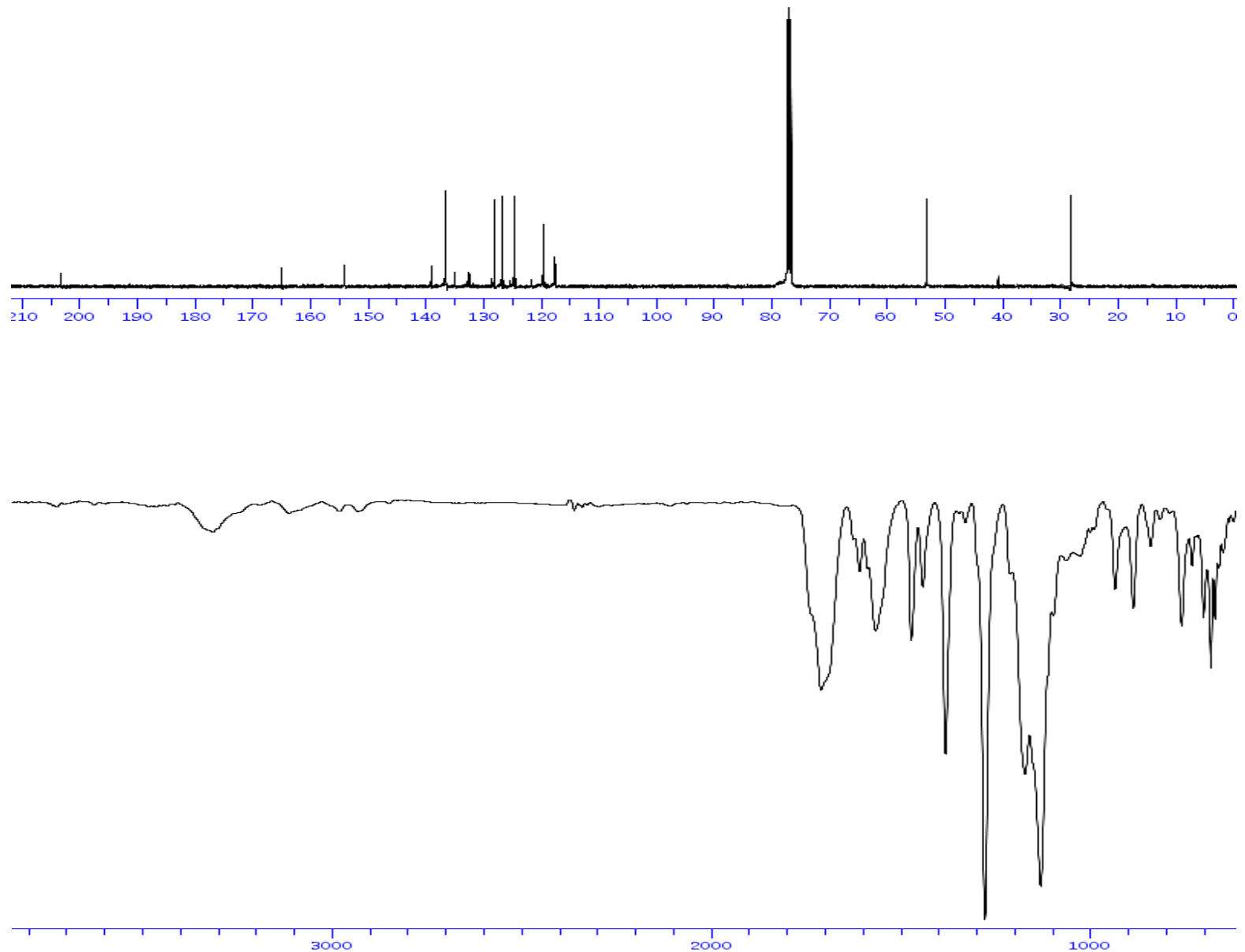
Methyl 2-ethynyl-1-oxo-2,3-dihydro-1H-indene-2-carboxylate (3a)

solvent: $>\mathrm{CDCl}>$
Frequency. $400.13 \mathrm{MHz}$

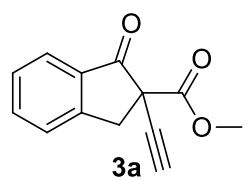

solvent: > CDCI3>
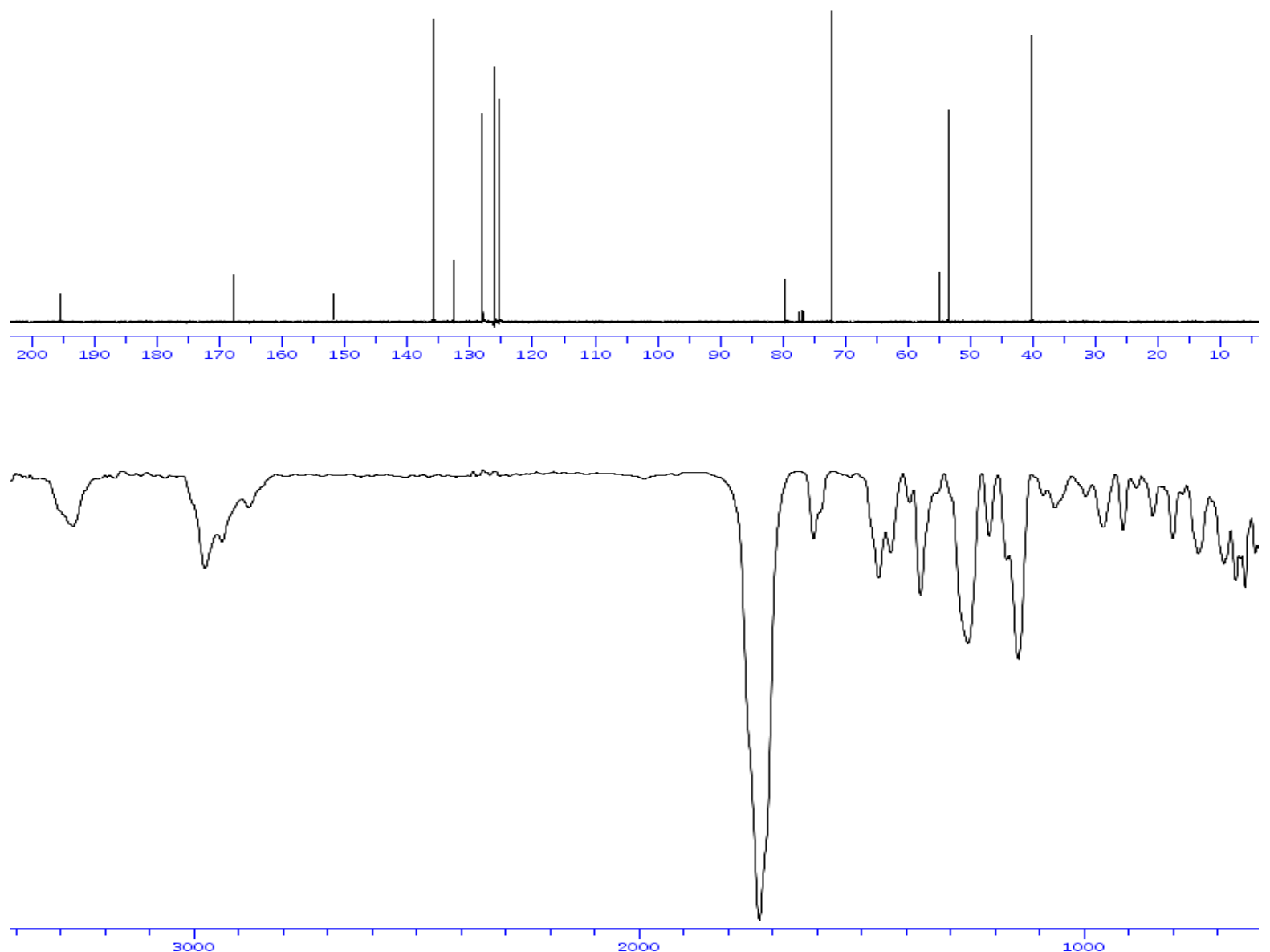
Tert-Butyl 2-ethynyl-1-oxo-2,3-dihydro-1H-indene-2-carboxylate (3b)

Solvent: $<\mathrm{CDCI}>$
Frequency, $400.13 \mathrm{MHz}$

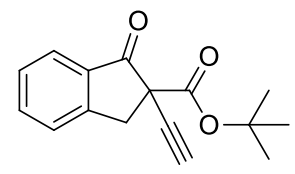

3b

1) 1

W. Wh

Sile

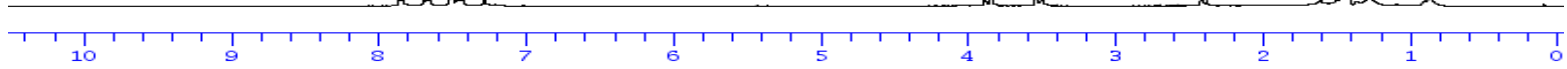

solvent: $<\mathrm{CDCI}>>$
Frequency.100.61 $1 \mathrm{MHz}$
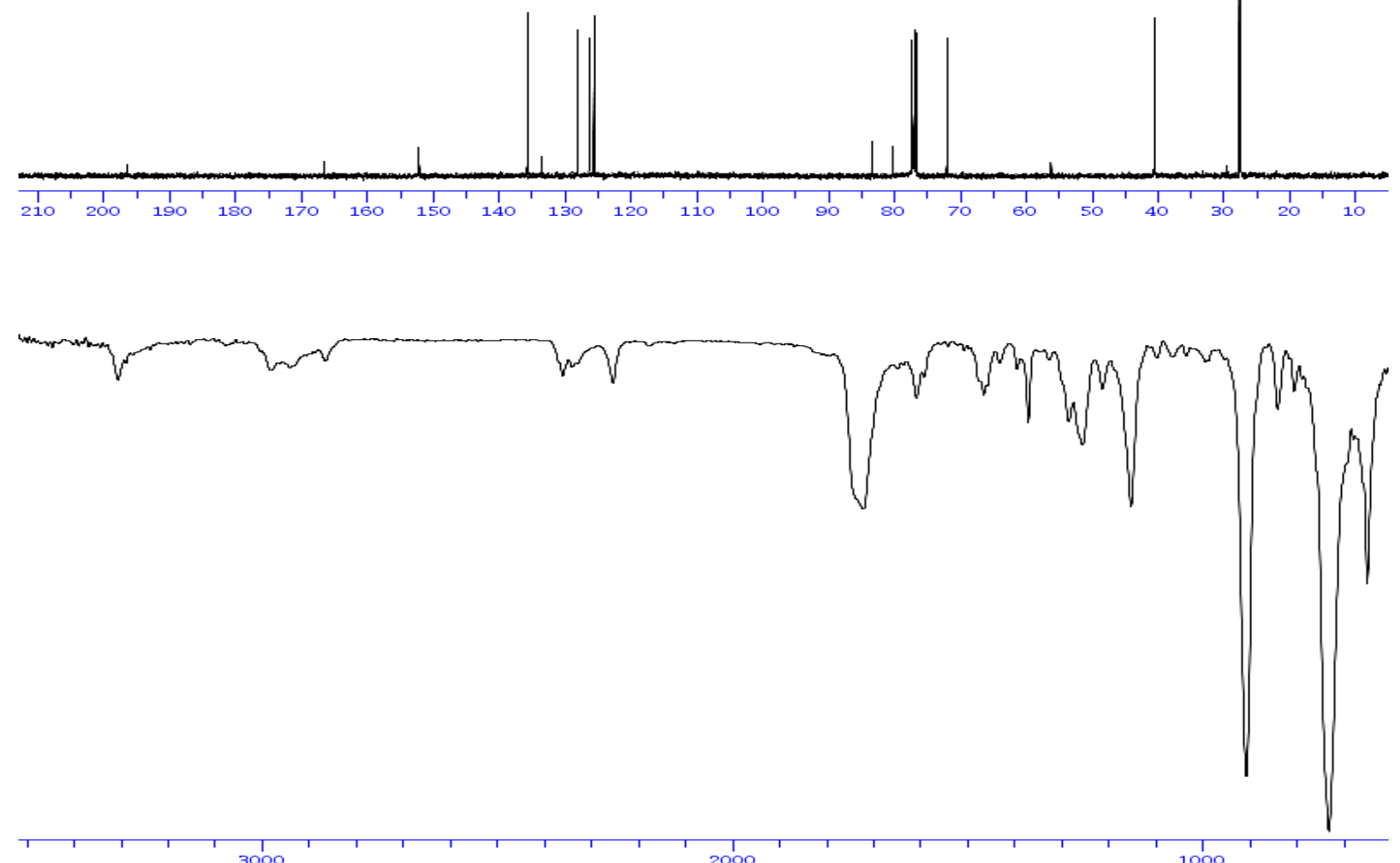
HPLC chromatogram for racemic and chiral tert-butyl 2-ethynyl-1-oxo-2,3-dihydro-1Hindene-2-carboxylate (3b)
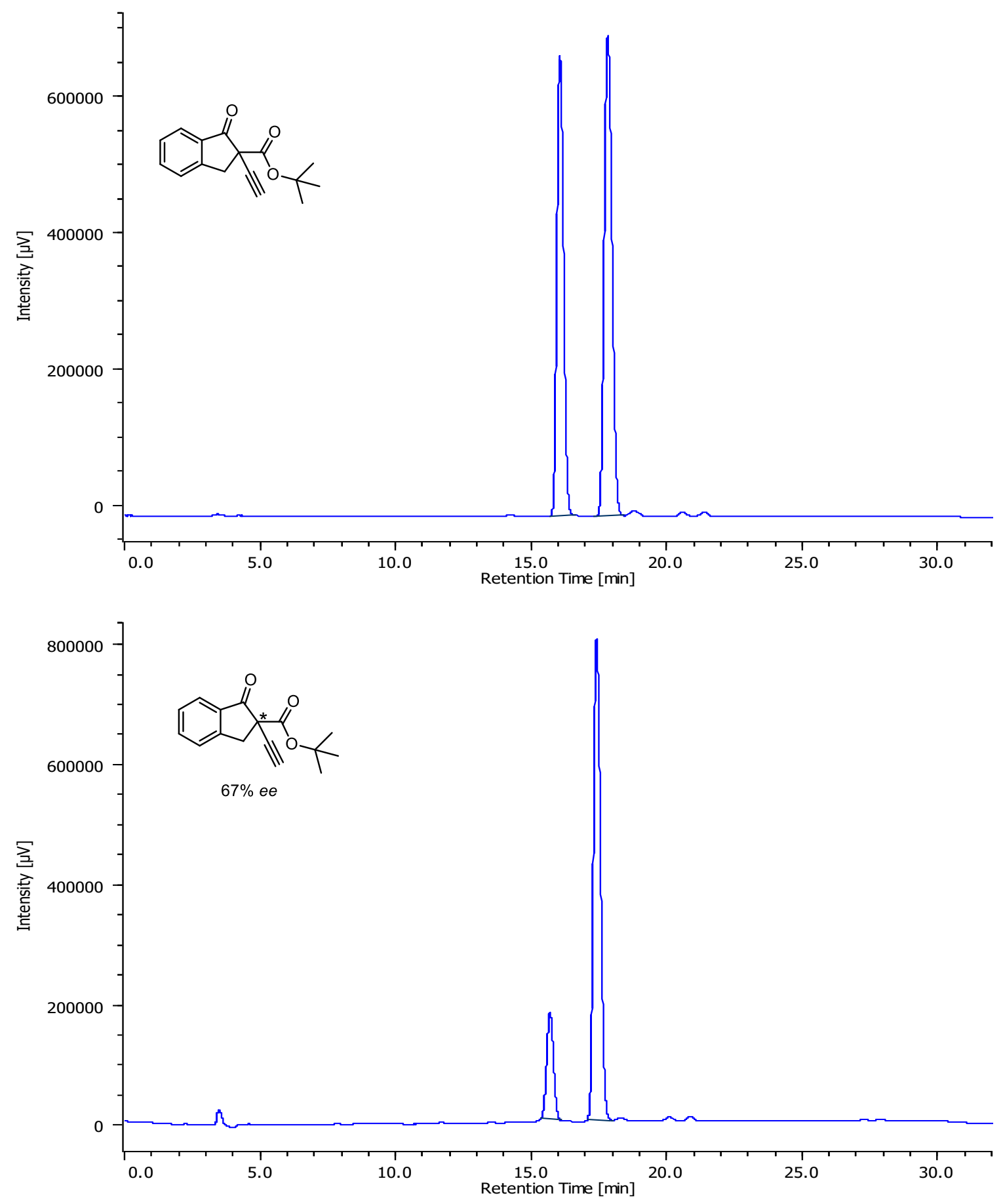
Tert-Butyl 1-oxo-2-((triisopropylsilyl)ethynyl)-2,3-dihydro-1 $H$-indene-2-carboxylate (3b')

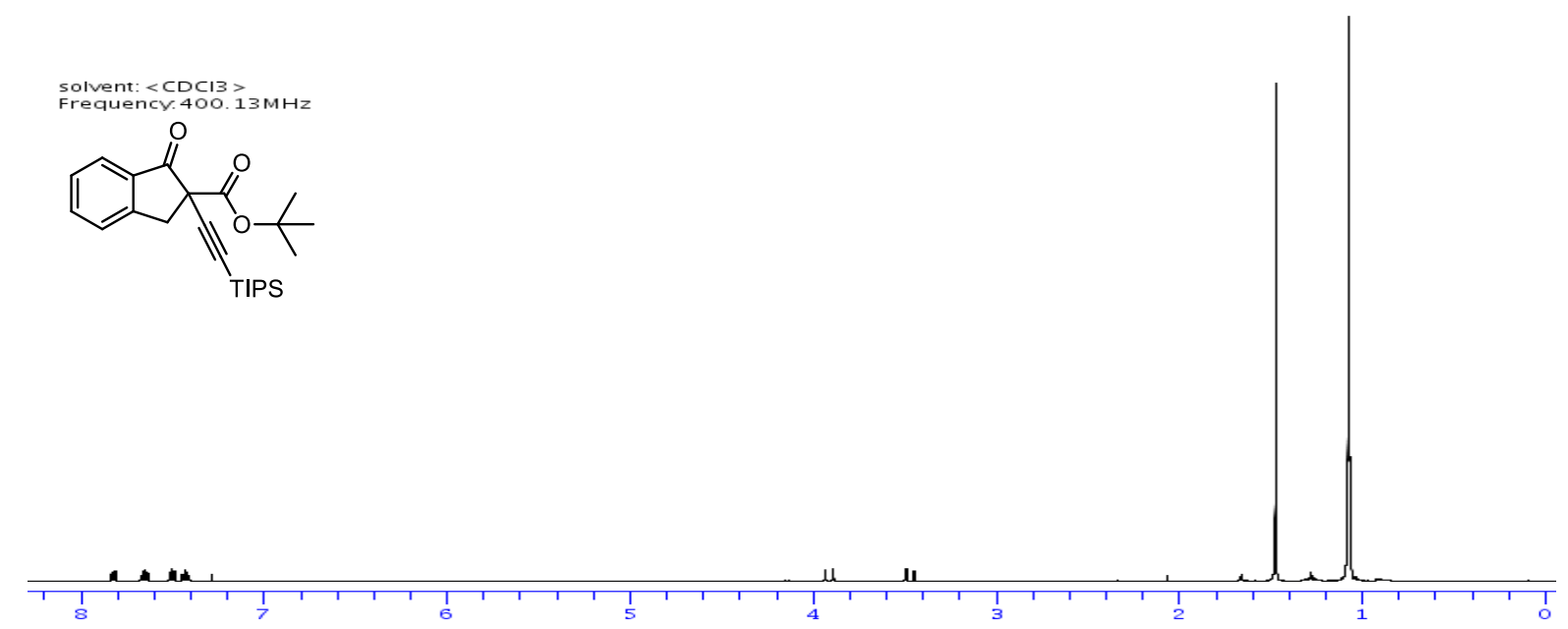

solvent: $\langle\mathrm{CDCI} 3\rangle$

$0.612769 \mathrm{MHz}$
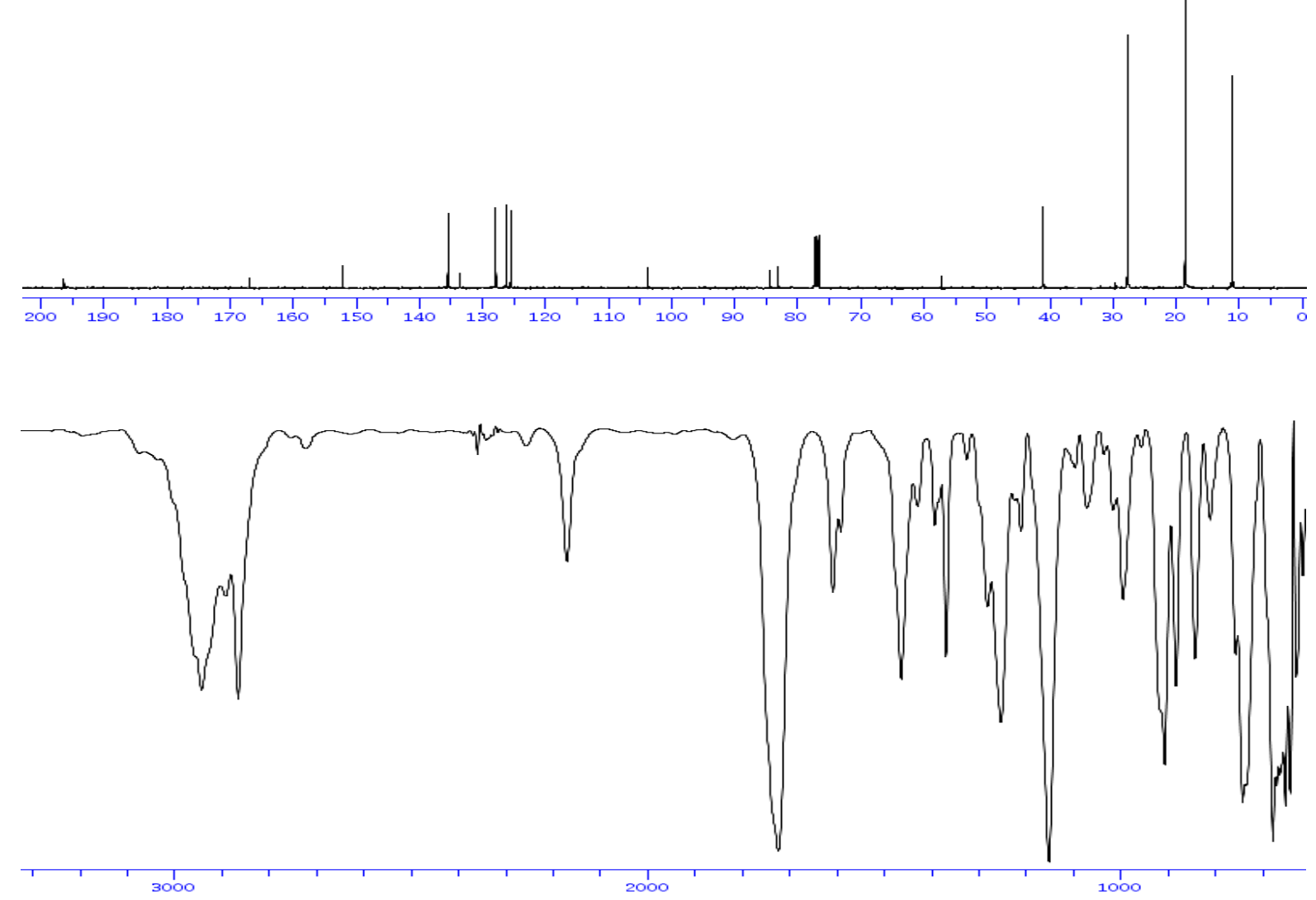
Solvent: $<\mathrm{CDCI}>$

forency. $400.13 \mathrm{MHz}$

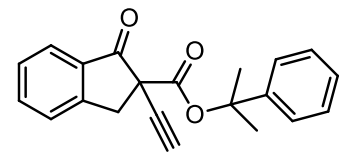

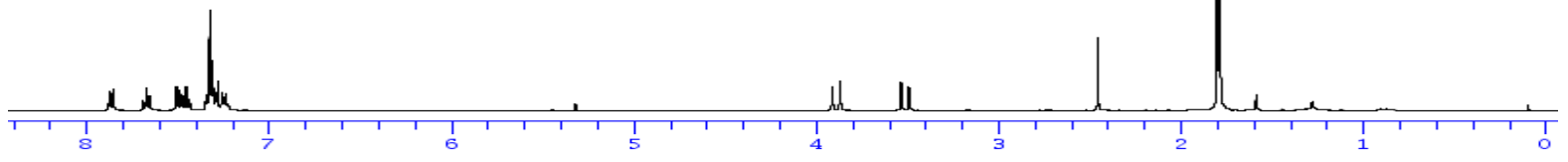

Solvent: $<C D C 13>$
Frequency. $100.612769 \mathrm{MHz}$
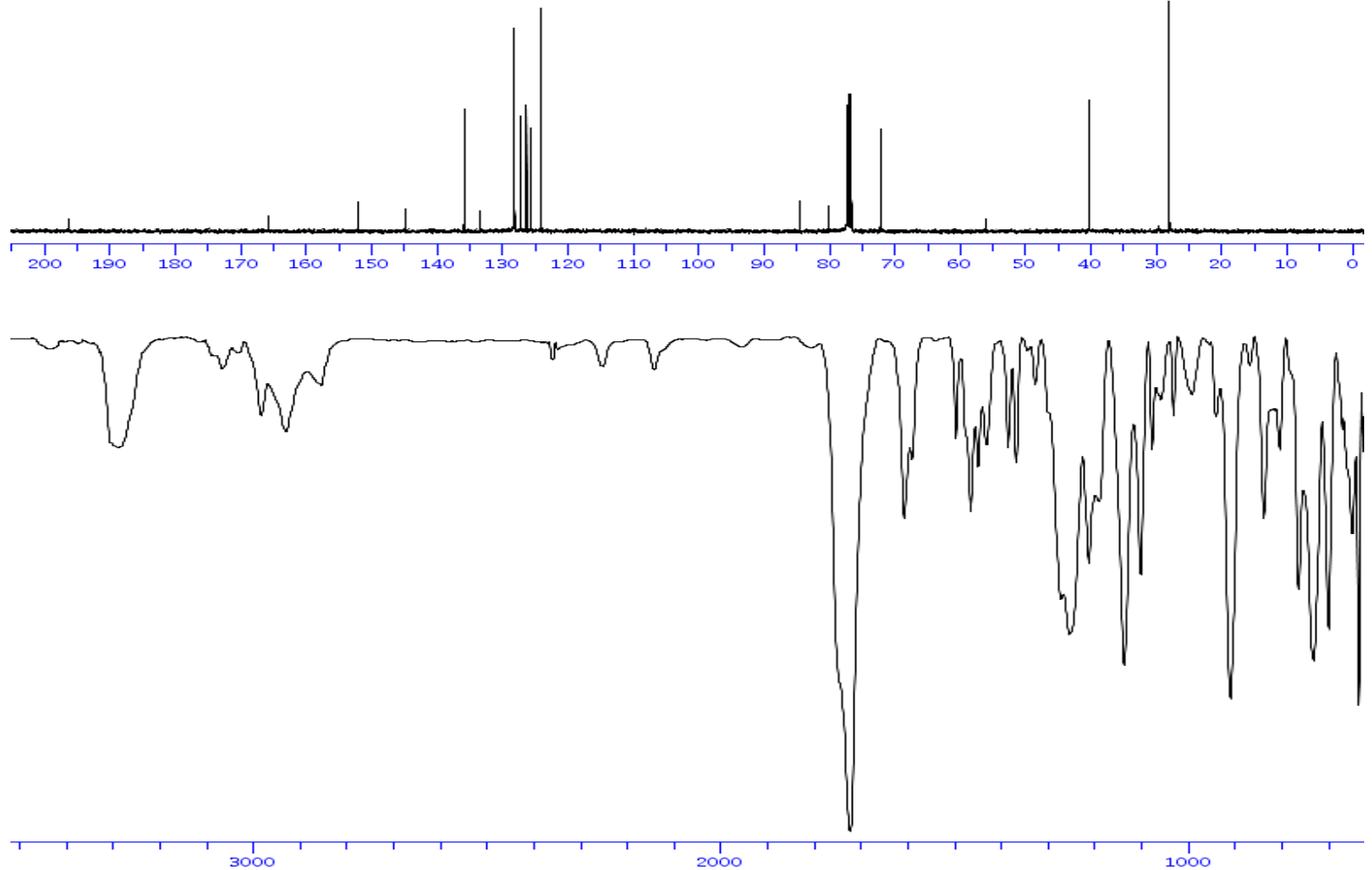
HPLC chromatogram for racemic and chiral 2-phenylpropan-2-yl 2-ethynyl-1-oxo-2,3dihydro-1H-indene-2-carboxylate (3c)
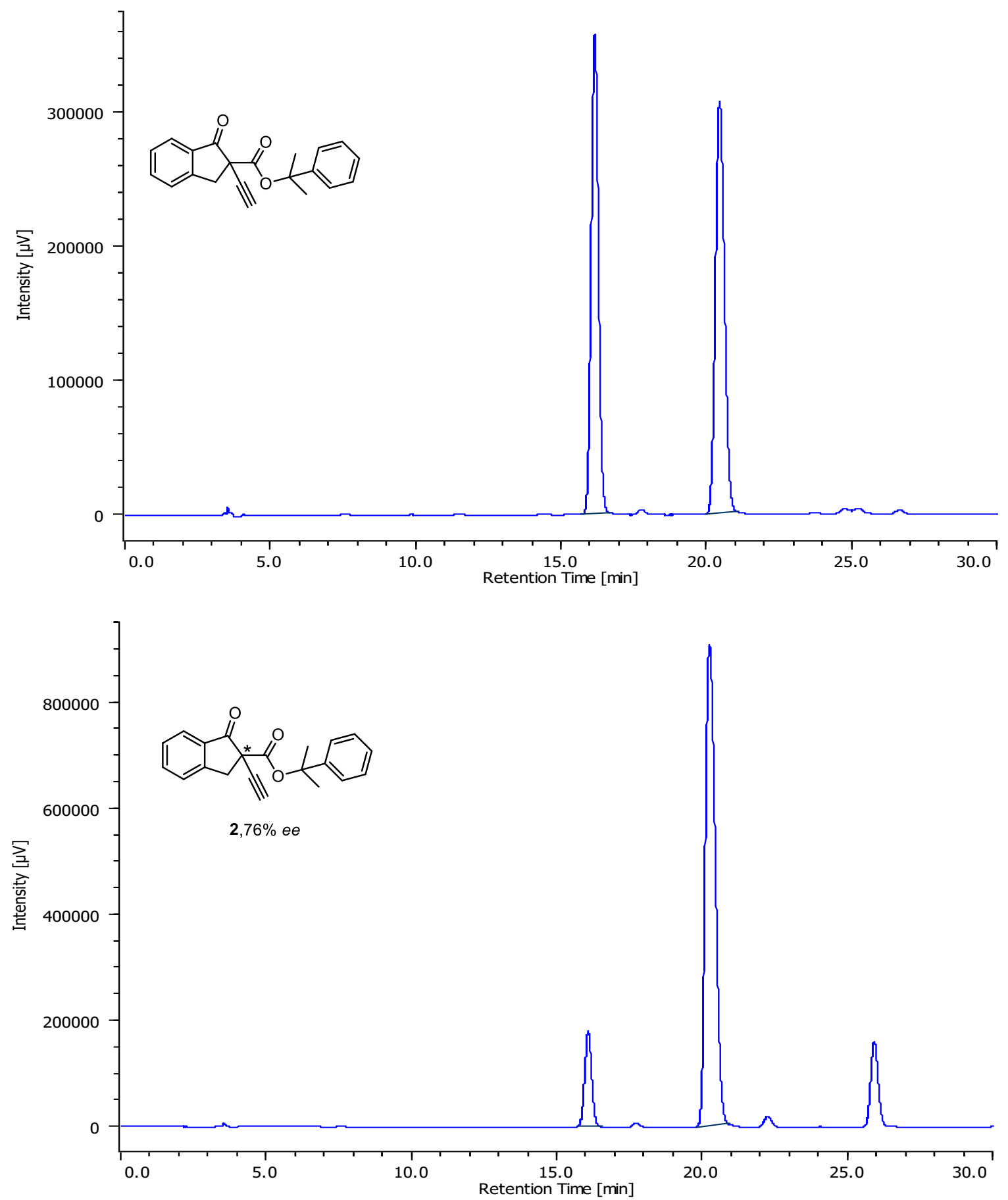

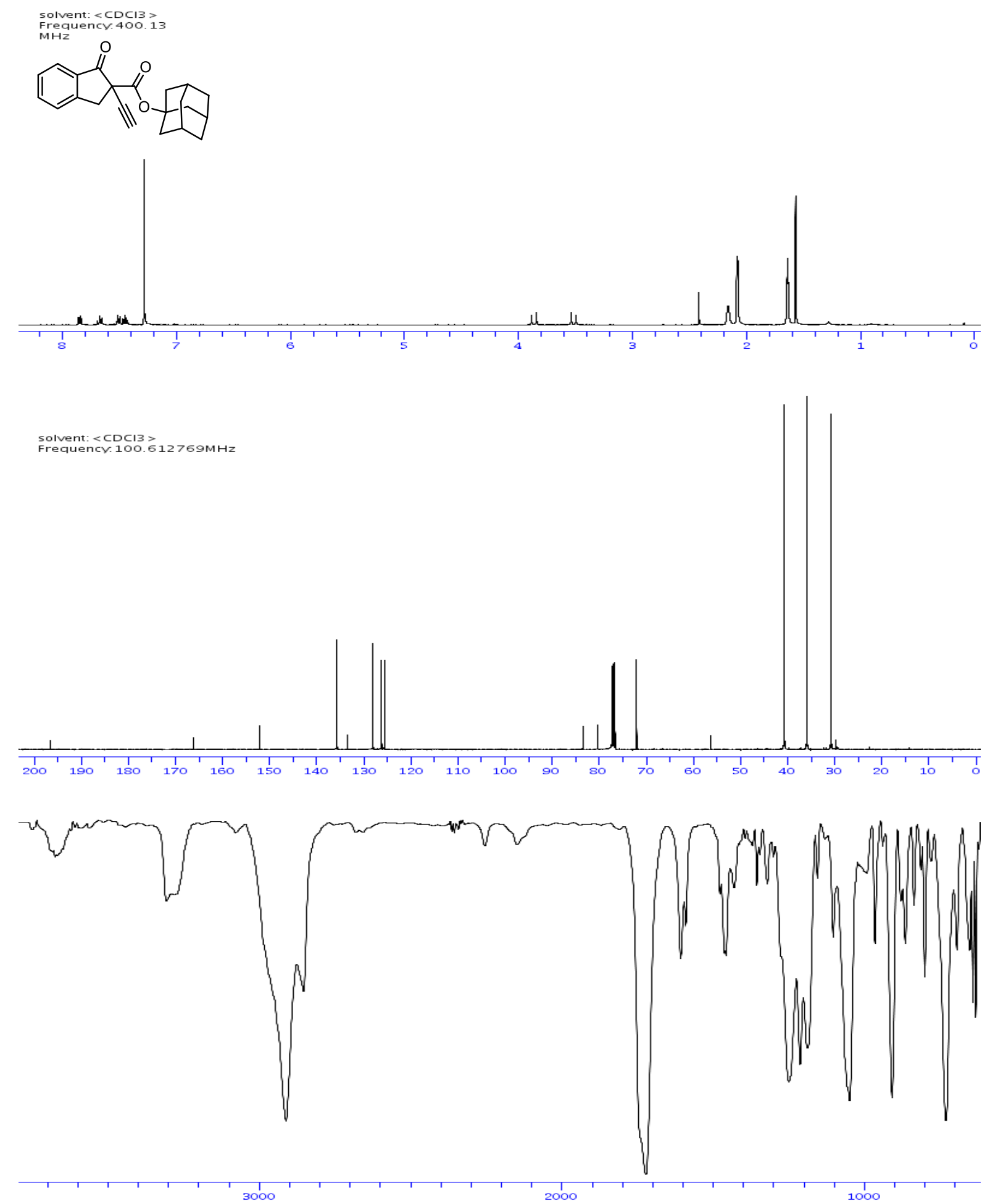
HPLC chromatogram for racemic and chiral 1-adamantan-1-yl 2-ethynyl-1-oxo-2,3dihydro-1H-indene-2-carboxylate (3d)
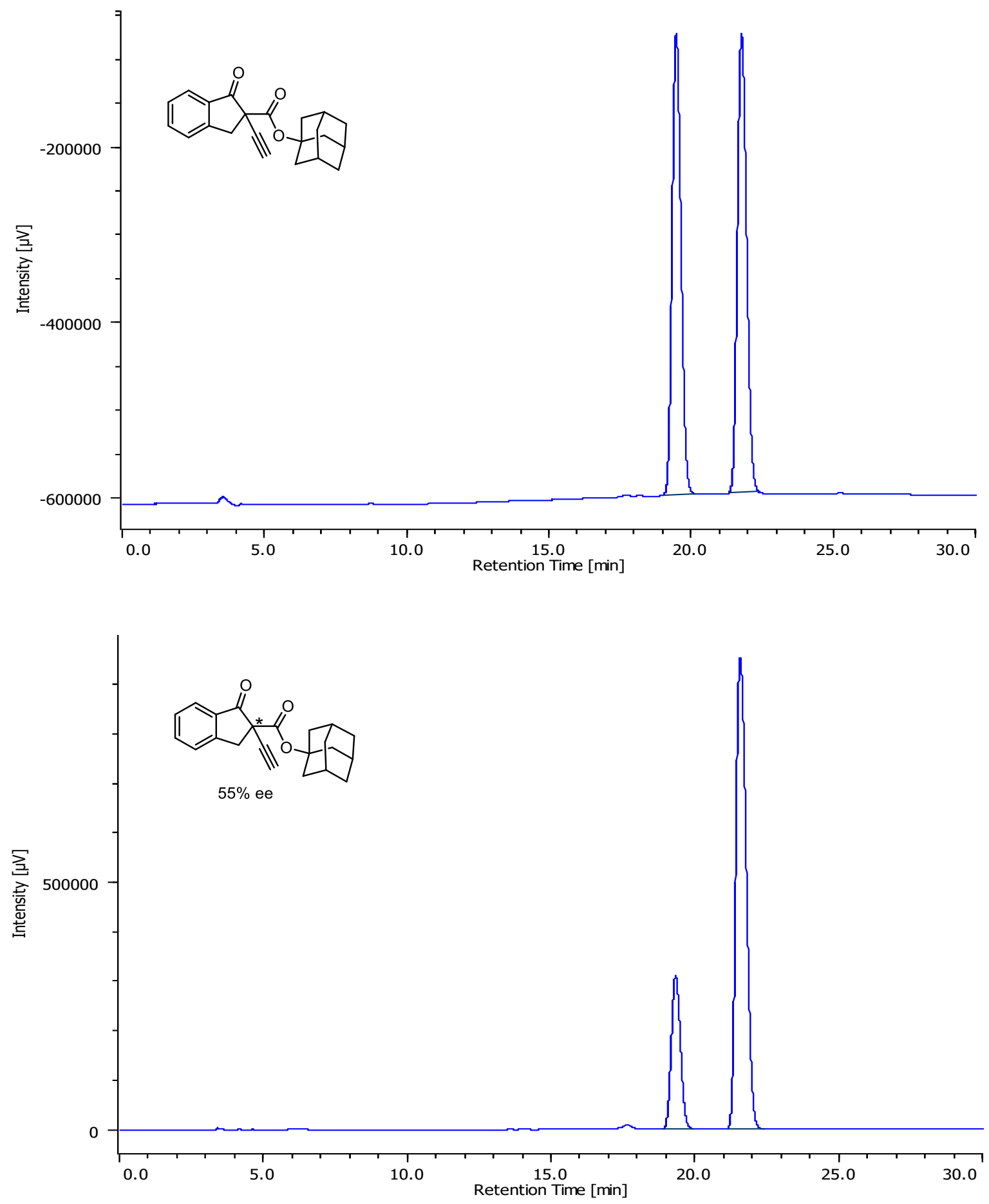
Anthracen-9-ylmethyl 2-ethynyl-1-oxo-2,3-dihydro- $1 H$-indene-2-carboxylate (3e)

solvent: $<\mathrm{CDCI} 3>$

Frequency. $400.13 \mathrm{MHz}$
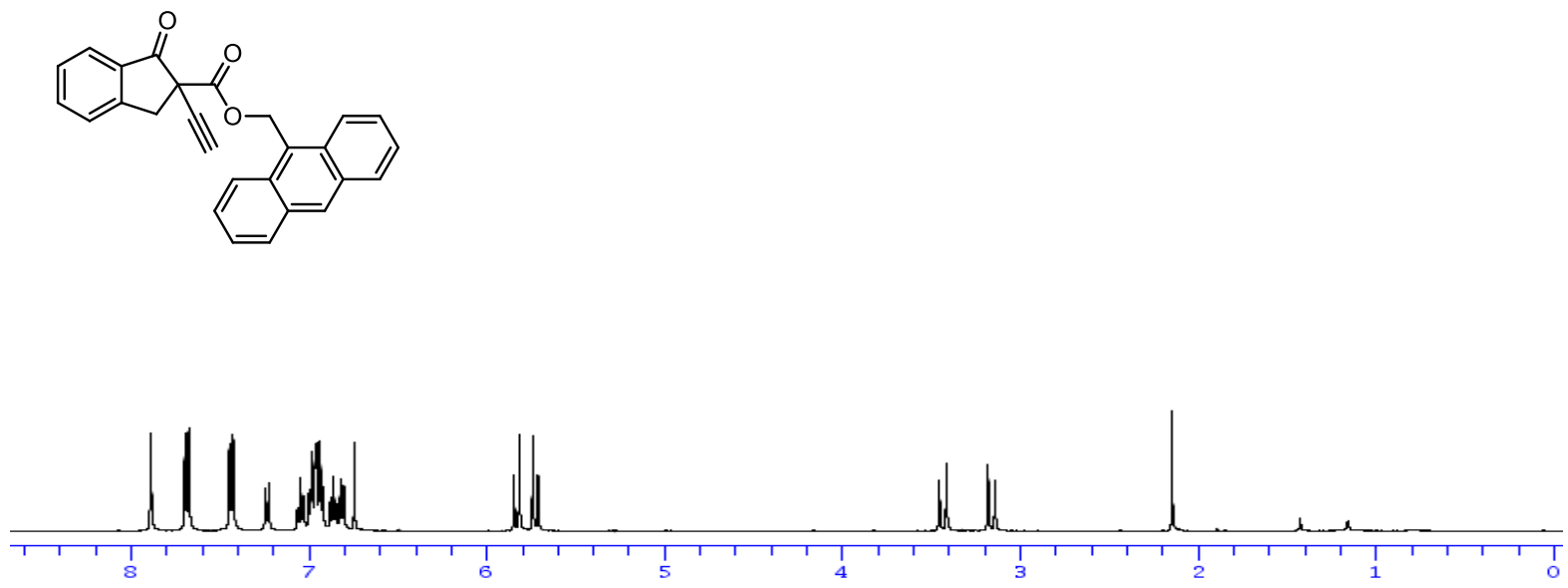

solvent: $<\mathrm{CDC13}>$
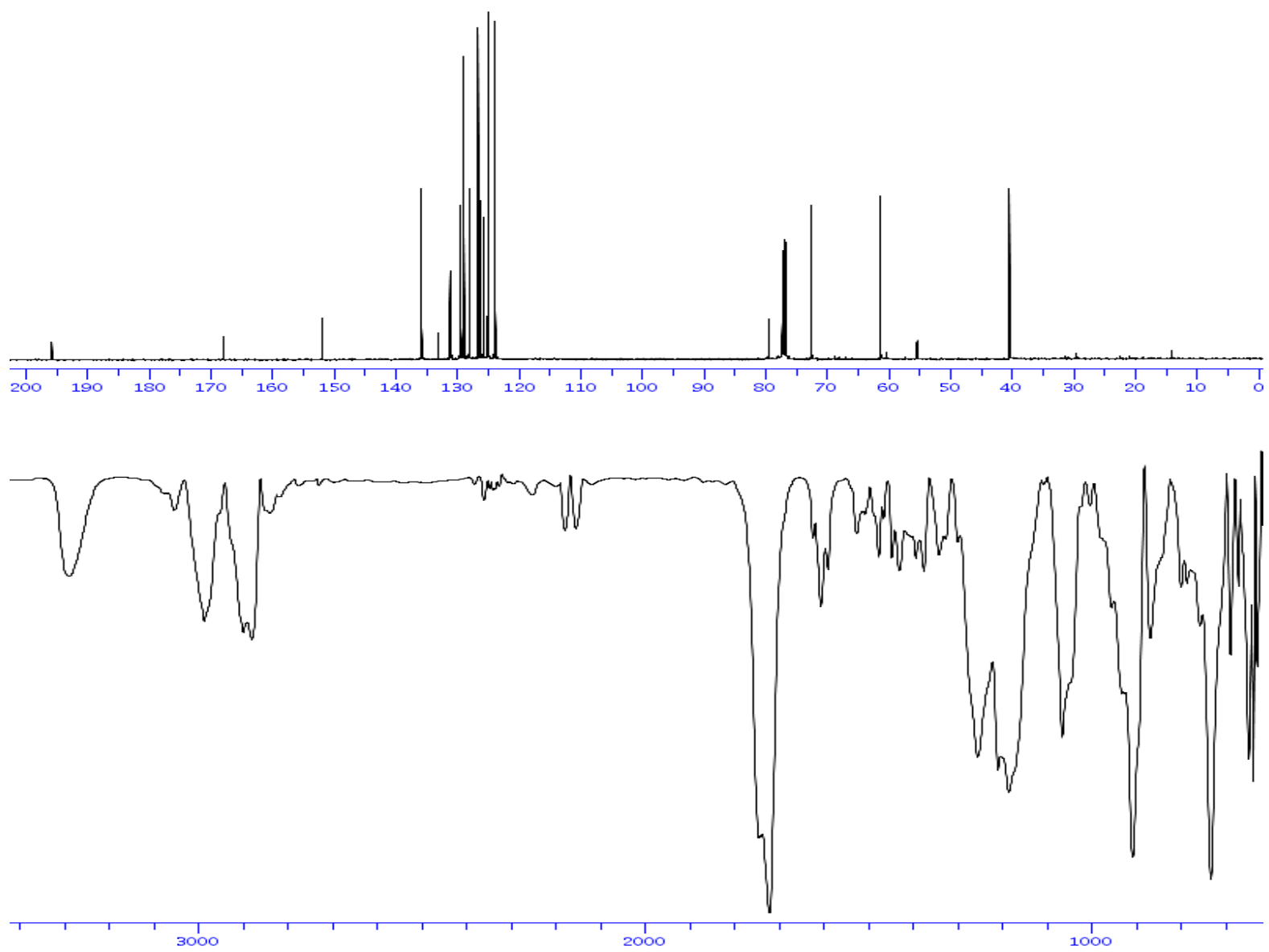

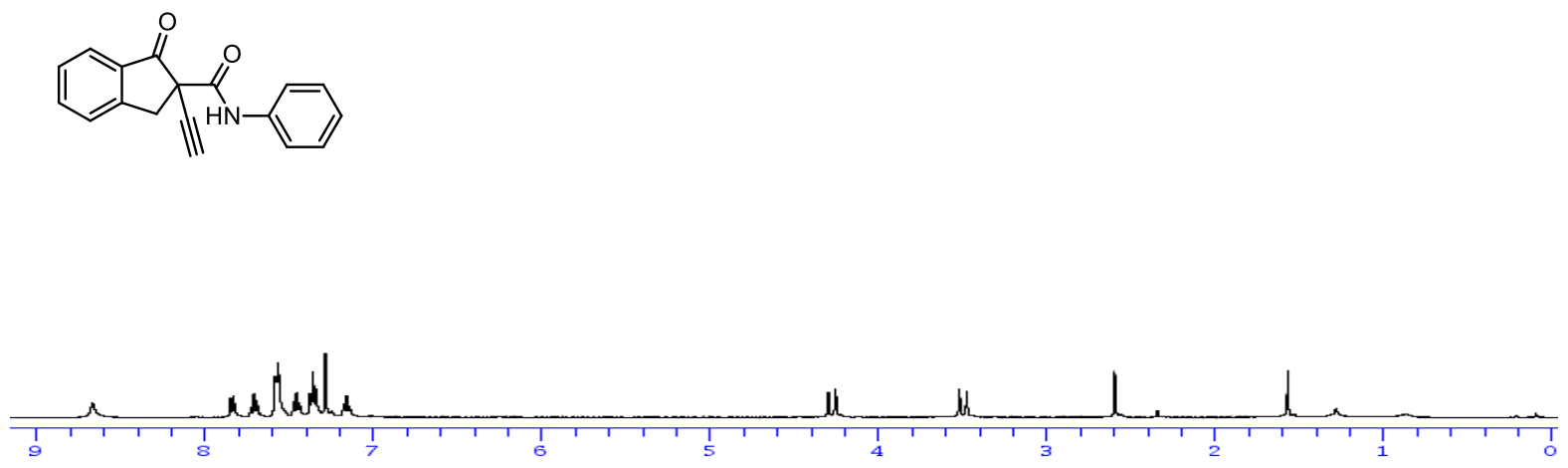

solvent: $<\mathrm{CDCI}>$
Frequency.100.612769M Hz
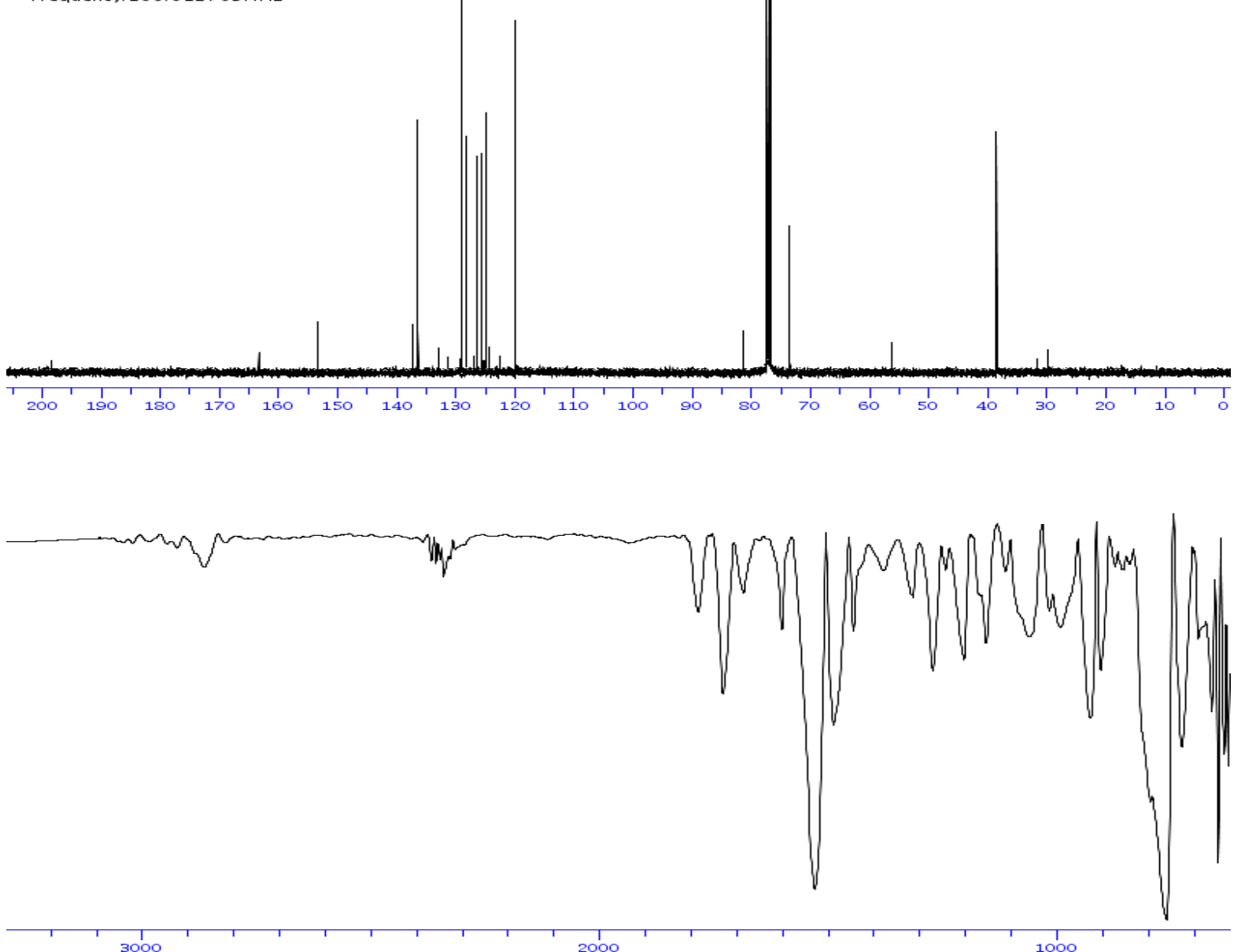
solvent: $<\mathrm{CDCl} 3>$
Frequency. $400.13 \mathrm{MHz}$
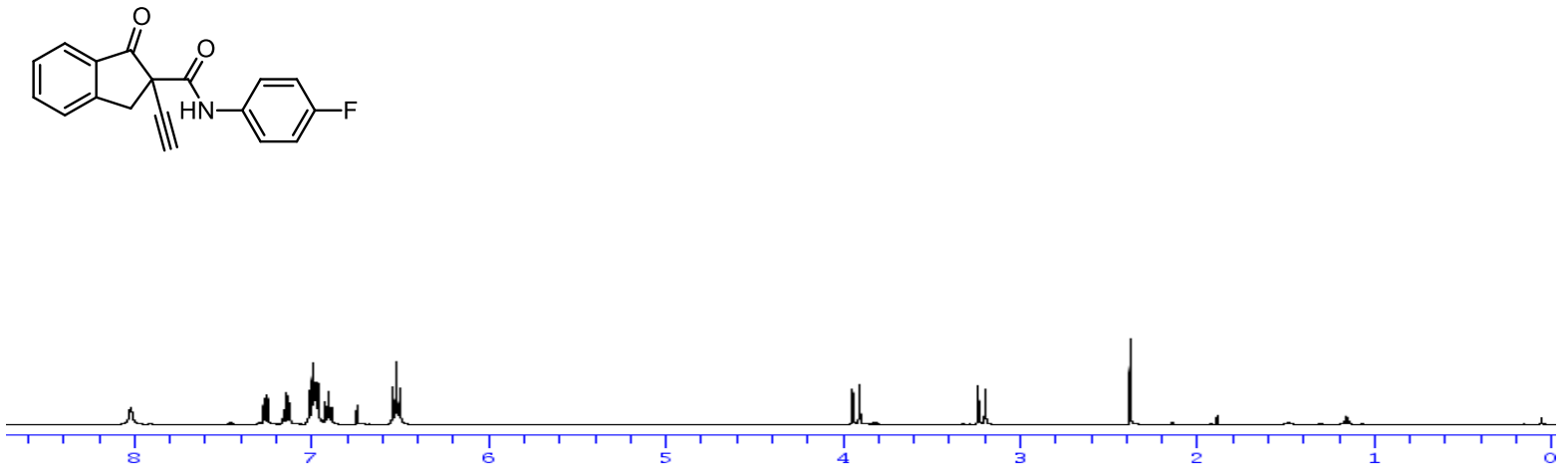

solvent: $<\mathrm{CDCI} 3$
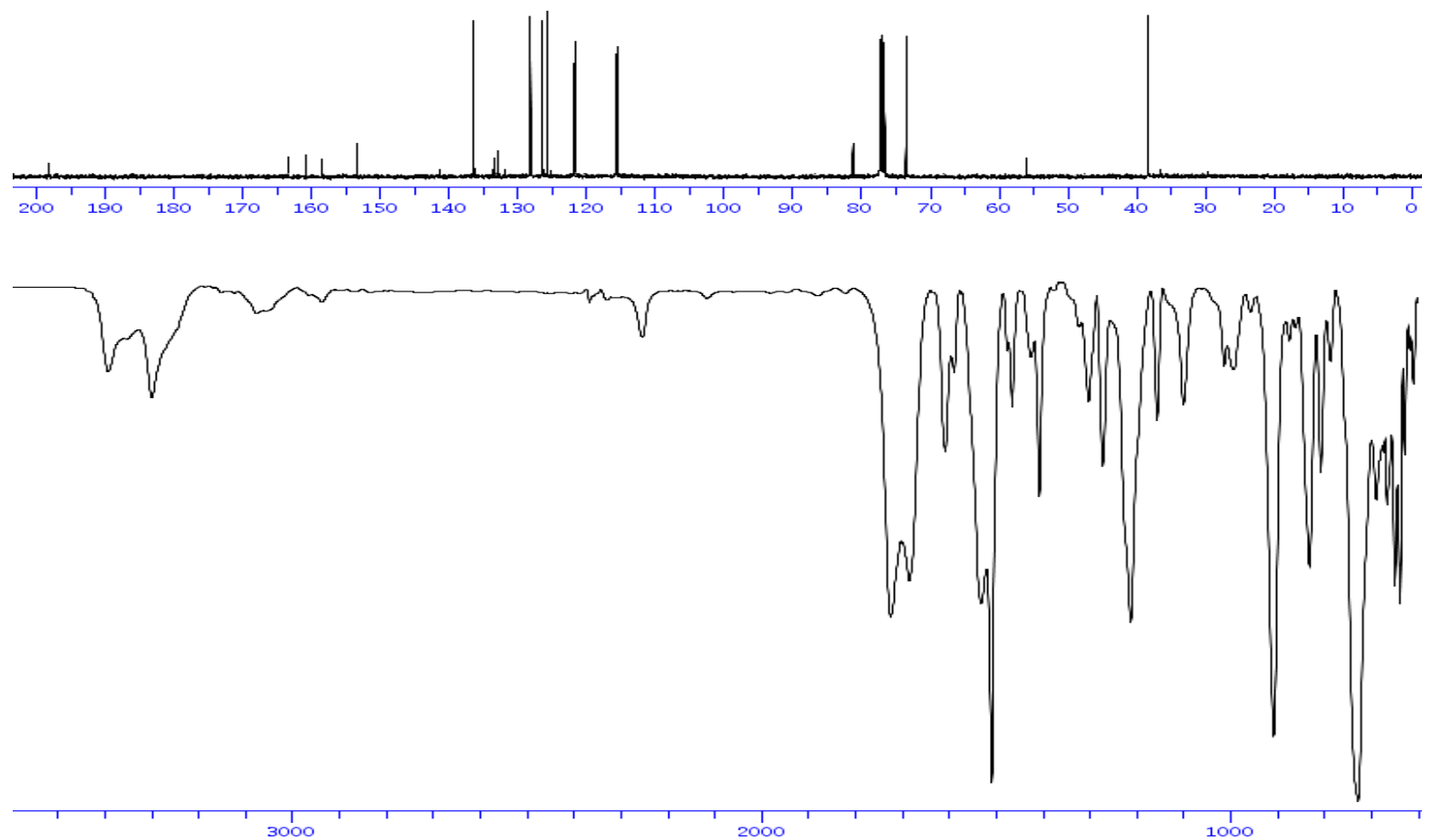
$N$-(3,5-bis(trifluoromethyl)phenyl)-2-ethynyl-1-oxo-2,3-dihydro-1 $H$-indene-2carboxamide (3h)<smiles>C#CC1(C(=O)Nc2cc(C(F)(F)F)cc(C(F)(F)F)c2)Cc2ccccc2C1=O</smiles>

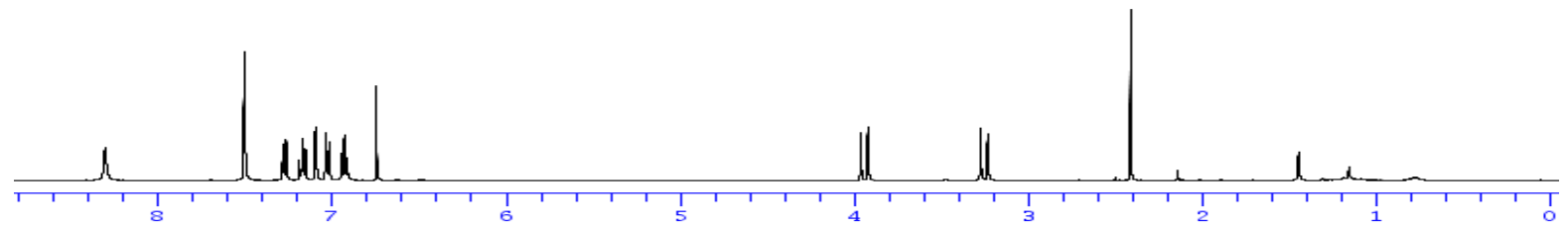

solvent: $<\mathrm{CDCl} 3>$
Frequency. $100.612769 \mathrm{MHz}$
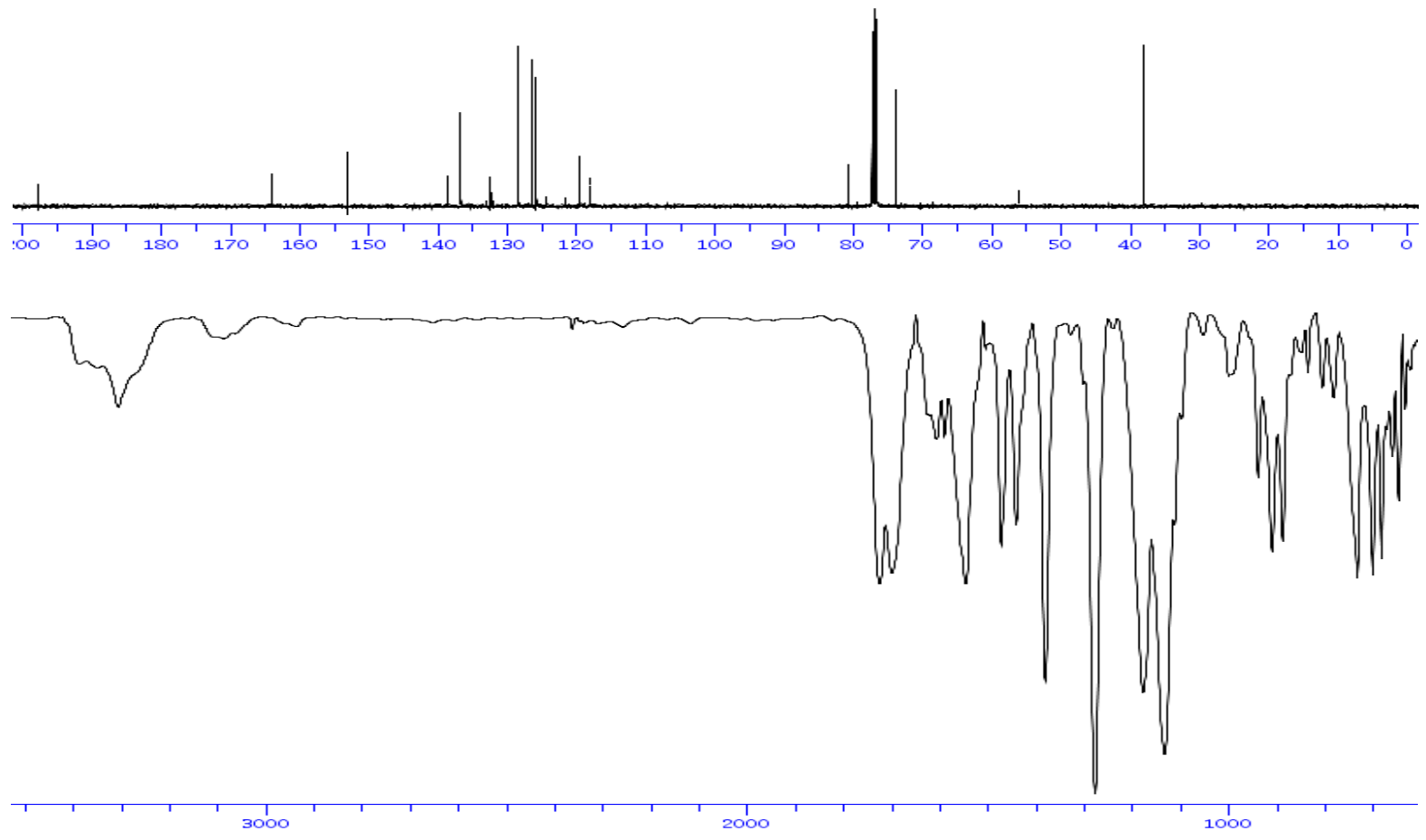